\title{
ASSESSMENT OF THE EFFECT \\ OF \\ HYDROELECTRIC POWER PLANTS' GOVERNOR SETTINGS \\ ON \\ LOW FREQUENCY INTER AREA OSCILLATIONS
}

A THESIS SUBMITTED TO

THE GRADUATE SCHOOL OF NATURAL AND APPLIED SCIENCES

OF

MIDDLE EAST TECHNICAL UNIVERSITY

BY

CIHHANGİR GENÇOĞLU

IN PARTIAL FULFILLMENT OF THE REQUIREMENTS FOR

THE DEGREE OF MASTER OF SCIENCE

IN

ELECTRICAL AND ELECTRONICS ENGINEERING

JULY 2010 
Approval of the thesis:

\section{ASSESSMENT OF THE EFFECT OF HYDROELECTRIC POWER PLANTS' GOVERNOR SETTINGS ON LOW FREQUENCY INTER AREA OSCILLATIONS}

submitted by CIHANGIR GENÇOĞLU in partial fulfillment of the requirements for the degree of Master of Science in Electrical and Electronics Engineering Department, Middle East Technical University by,

Prof. Dr. Canan Özgen

Dean, Graduate School of Natural and Applied Sciences

Prof. Dr. İsmet Erkmen

Head of Department, Electrical and Electronics Engineering

Prof. Dr. A. Nezih Güven

Supervisor, Electrical and Electronics Engineering Dept., METU

\section{Examining Committee Members}

Prof. Dr. Nevzat Özay

Electrical and Electronics Engineering Dept., METU

Prof. Dr. A. Nezih Güven

Electrical and Electronics Engineering Dept., METU

Prof. Dr. Arif Ertaş

Electrical and Electronics Engineering Dept., METU

Prof. Dr. Muammer Ermiş

Electrical and Electronics Engineering Dept., METU

Dr. Osman Bülent Tör

Tübitak - UZAY 
I hereby declare that all information in this document has been obtained and presented in accordance with academic rules and ethical conduct. I also declare that, as required by these rules and conduct, I have fully cited and referenced all material and results that are not original to this work.

Name, Last name : Cihangir Gençoğlu

Signature 


\author{
ABSTRACT \\ ASSESSMENT OF THE EFFECT OF HYDROELECTRIC POWER PLANTS \\ GOVERNOR SETTINGS ON LOW FREQUENCY INTER AREA \\ OSCILLATIONS \\ Gençoğlu, Cihangir \\ MS, Department of Electrical and Electronics Engineering \\ Supervisor: Prof. Dr. Ali Nezih Güven
}

July 2010, 155 Pages

The Turkish TSO (TEIAS) has been leading a project that aims the synchronous interconnected operation of the Turkish Power System and the ENTSO-E CESA (former UCTE) System. For this purpose, this study concentrates on the specific problems related to the electromechanical systems of large size hydroelectric power plants regarding low frequency inter area oscillations, which are prone to occur once the interconnected operation is established. The expected frequency of inter area oscillations after interconnected operation is close to $0.15 \mathrm{~Hz}$, which is in the frequency range of the speed governing structures of turbines, as explained in the first two sections of the thesis. In the third section, the nonlinear turbine governor model used throughout the study is explained. In the following part, the governor parameter tuning study with regard to the defined performance objectives is explained. Afterwards, the effect of the retuned governor settings of the sample hydroelectric power plants on a simple multi machine power system is shown. Following that, the system wide effect of removing the sources of negative damping, which are strongly dependent on the governor settings of the major hydroelectric power plants of the Turkish Power System, is shown. In the final part, conclusions are made on the operation of the hydroelectric power plants regarding the frequency stability of the system after synchronous interconnected operation of the 
Turkish Power System and the ENTSO-E CESA System.

Keywords -Hydroelectric Power Generation, Interconnected Power Systems, Load Frequency Control, Power System Dynamic Stability, Power System Modeling. 


\title{
öz
}

\section{HIDDROELEKTRİK SANTRALLERIN HIZ REGÜLATÖRÜ AYARLARININ DÜŞÜK FREKANSLI BÖLGELER ARASI SALINIMLARA ETKISINIIN INCELENMESI}

\author{
Gençoğlu, Cihangir \\ Yüksek Lisans, Elektrik ve Elektronik Mühendisligi Bölümü \\ Tez Yöneticisi: Prof. Dr. Ali Nezih Güven
}

Temmuz 2010, 155 Sayfa

Türkiye iletim sistemi operatörü TEIAS, Türkiye İletim Sistemi ve ENTSO-E CESA (eski adıyla UCTE) sistemlerinin senkron paralel bağlantısını amaçlayan bir proje yürütmektedir. Bu amaçla yürütülen çalışmaların ışı̆̆ında, bu çalışmada, Türkiye Elektrik Sistemi'ndeki büyük güçteki hidroelektrik santrallerin hız regülatörü ayarlarının, bağlantı sonucu oluşması muhtemel bölgeler arası salınımlara etkisi üzerine yoğunlaşılmıştır. İki iletim sisteminin senkron parallel bağlantısının ardından oluşması muhtemel bölgeler arası salınımların frekansı $0.15 \mathrm{~Hz}$ civarındadır ki, bu çalışmanın ilk iki bölümünde gösterildiği üzere, bahsedilen salınım frekansı, üretim tesislerinin hız regülatörleri ve türbinlerinin de tepkisel olduğu frekans bandına denk gelmektedir. Çalışmanın üçüncü bölümünde, bölgeler arası salınımların sistem frekans kararlılığı üzerindeki olumsuz etkilerini asgariye indirmeyi amaçlayan hidroelektrik santral hız regülatörü ayarlarına ulaşmak için kullanılan türbin ve hız regülatörü modelleri açıklanmıştır. Çalışmanın dördüncü bölümünde, sistemin üretim tüketim dengesizliği durumundaki davranışını da gözeterek, bölgeler arası salınımların sistem frekans kararlılığı üzerindeki olumsuz etkilerini asgariye indirmeyi amaçlayan hidroelektrik santral hız regülatörü ayarlarının tespit edilme yöntemi açıklanmış ve bu yöntemle elde edilen hız regülatörü ayarlarının frekans 
kararlılığı üzerindeki etkisi çok makineli bir güç sisteminde gösterilerek, önerilen ayarların sistem işletimine uygun olduğu sonucuna varılmıştır. Sonrasında, Türkiye Elektrik Sistemi'ndeki bütün büyük güçteki hidroelektrik santraller için, önerilen yöntemle elde edilen hız regülatörü ayarlarının sistem genelindeki sönümlendirme performansını arttırıcı etkisi gösterilmiştir. Çalışmanın son bölümünde, ENTSO-E CESA sistemi ile senkron parallel bağlantı sonrasında, hidroelektrik santrallerin sistem frekans kararlılığı gözetilerek işletilmesi noktasında elde edilen sonuçlar ifade edilmiştir.

Anahtar kelimeler -Hidroelektrik Santraller, Enterkonnekte Güç Sistemleri, Yük Frekans Kontrolü, Güç Sistemlerinin Dinamik Kararlılığı, Güç Sistemi Modelleme. 
To My Parents

My Brother

and My Beloved Sweetie 


\section{ACKNOWLEDGMENTS}

First of all, I would like to thank my supervisor, Prof. Dr. Ali Nezih Güven, for his guidance, advice, criticism and encouragement throughout the study.

I would also like to express my deepest gratitude to my family for their unconditional support, love and encouragement throughout my life.

I also wish to thank all members of the Power Systems Group of TÜBİTAK-UZAY and TEIAS Europe Transmission Coordination Division, for their support, intimate friendship and for creating an excellent working environment. I especially thank my colleagues Osman Bülent Tör, Oğuz Yılmaz and Mahmut Erkut Cebeci, who have encouraged and advised me throughout the M.S. study.

Finally, I would like to thank Cansu Gündüz, only to whom my heart belongs, for bestowing her whole and true love upon me. 


\section{TABLE OF CONTENTS}

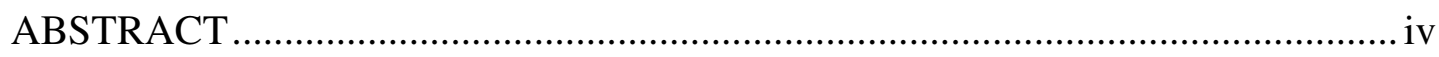

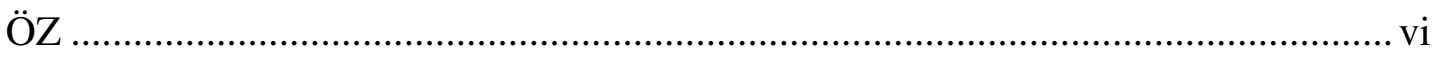

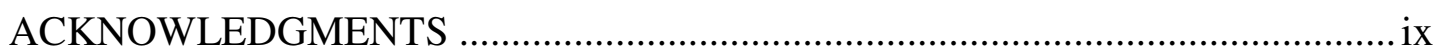

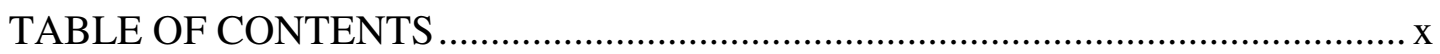

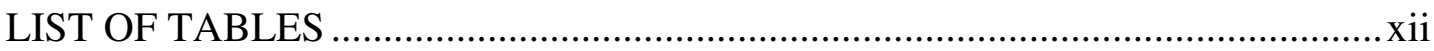

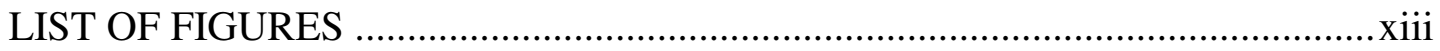

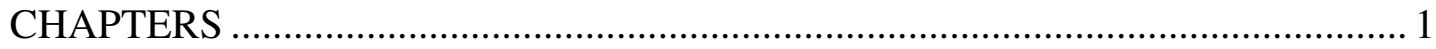

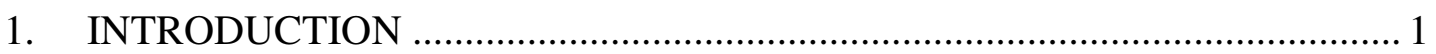

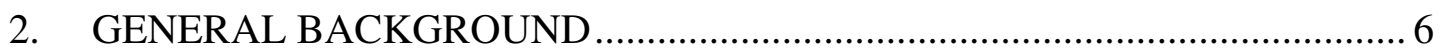

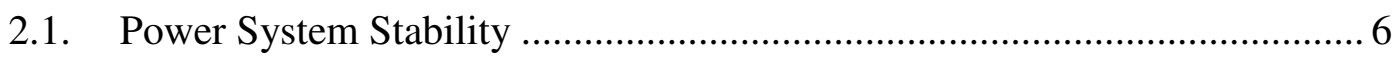

2.2. Classification of Power System Stability .................................................. 8

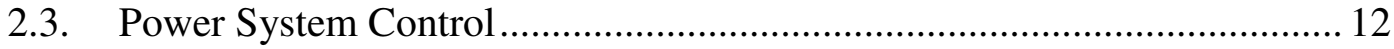

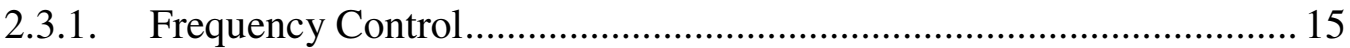

2.3.1.1. Primary Frequency Control ................................................... 18

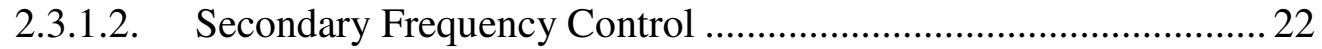

2.3.1.3. Tertiary Frequency Control ....................................................... 27

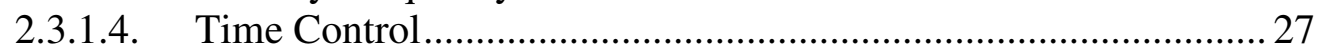

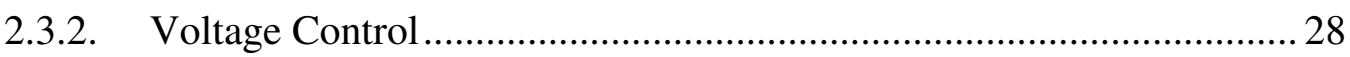

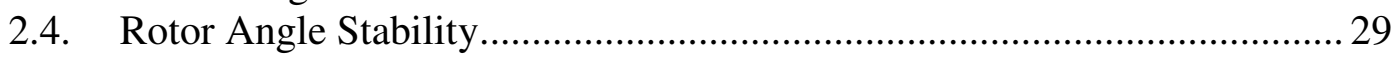

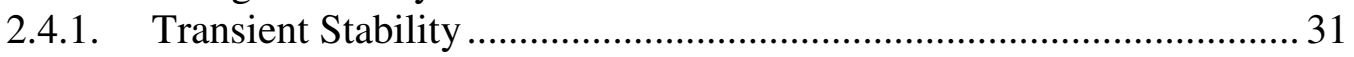

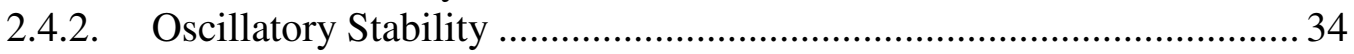

2.4.2.1. Oscillatory Stability Phenomenon ............................................. 35

2.4.2.2. Local Oscillations and Inter Area Oscillations ........................... 37

2.4.2.3. The Effect of Rotor Flux Linkage Variation ............................... 38

2.4.2.4. The Effect of AVR on Damper Windings ................................... 39

2.4.2.5. Power System Stabilizers............................................................. 42

2.5. Problem Definition ................................................................................ 43

2.5.1. The Current Practice of Frequency and Voltage Control in Turkish

Power System .............................................................................................. 43

2.5.2. The Expected Mode of Inter Area Oscillations after Interconnected

Operation and Expected Problems .................................................................... 44

2.5.3. The Counter Measures against the Risk of Undamped Oscillations.... 49 


\section{MODELING OF THE HYDROELECTRIC UNITS AND THE}

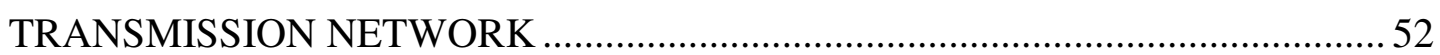

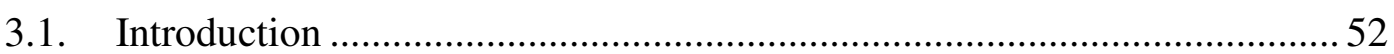

3.2. Modeling Assumptions and Mathematical Preliminaries ..........................5 53

3.2.1. Solution of Matrix Differential Equations .......................................... 55

3.2.1.1. Eigenvalues and Eigenvectors ..................................................56

3.2.1.2. Solution of Matrix Differential Equations ................................. 58

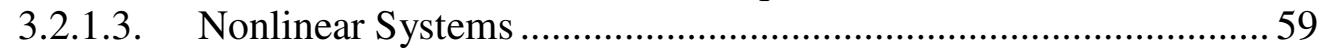

3.2.2. Periodic Solutions of Nonlinear Systems with Informal Arguments ... 60

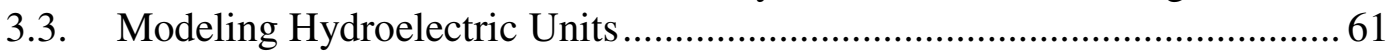

3.3.1. The Structure of a Hydroelectric Power Plant ......................................62 62

3.3.2. Electromechanical System Modeling.................................................6 63

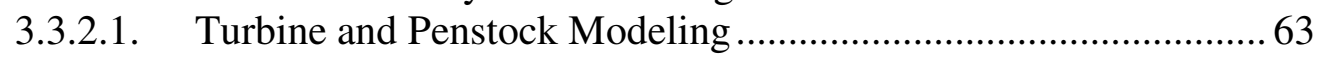

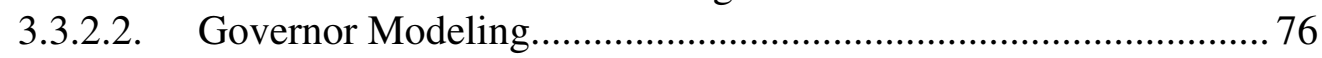

3.3.3. Generator and Excitation System Modeling .................................... 79

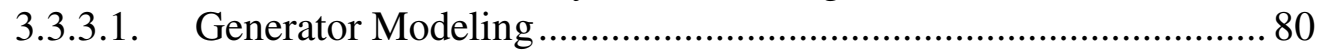

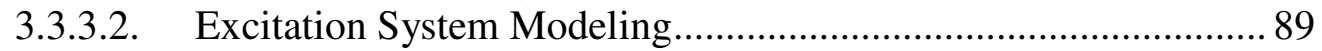

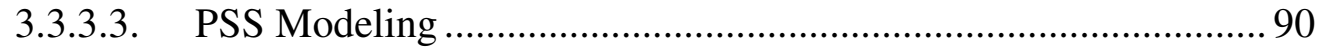

3.4. Network Modeling for Oscillatory Stability Studies................................ 91

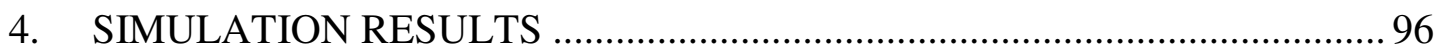

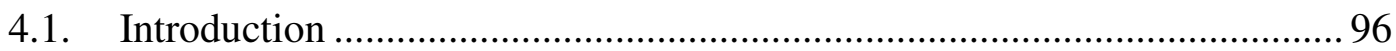

4.2. Sir Hydroelectric Power Plant Governor Retuning Study......................... 98

4.2.1. Governor Tuning Study ................................................................ 98

4.2.1.1. Modeling of a Unit of Sir Hydroelectric Power Plant ............... 100

4.2.1.2. Evaluation of the Existing Governor Settings .......................... 103

4.2.1.3. The Proposed Governor Settings ............................................... 114

4.2.2. Assessment of the Effectiveness of the Proposed Governor Settings in

Multi Machine Network ............................................................................. 124

4.3. Karakaya Power Plant Modeling Study .................................................. 128

4.3.1. Governor Tuning by Single Machine Infinite Busbar Model ............ 129

4.3.1.1. Evaluation of the Existing Governor Settings .......................... 129

4.3.1.2. The Proposed Governor Settings ................................................ 135

4.3.2. Assessment of Effectiveness of the Proposed Governor Settings in

Multi Machine Network.............................................................................. 140

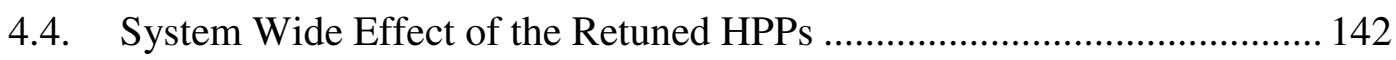

4.4.1. Load Flow Scenario ....................................................................... 143

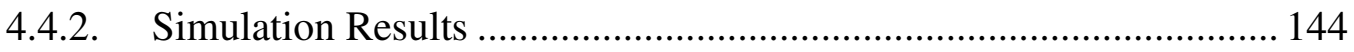

5. CONCLUSIONS AND FUTURE WORK …................................................ 147

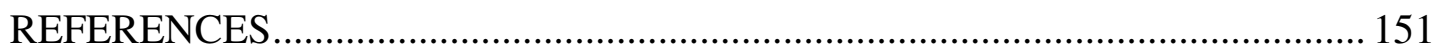




\section{LIST OF TABLES}

\section{TABLES}

Table 2-1: The List of Most Poorly Damped Modes Expected after Interconnected Operation.....

Table 2-2: The Priority List of Hydroelectric Power Plants ......................................50

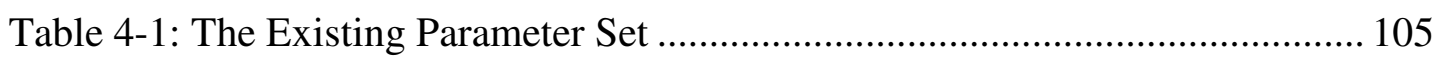

Table 4-2: The Proposed Parameter Set (1st Parameter Set) .................................. 114

Table 4-3: The Proposed Parameter Set (2nd Parameter Set) ................................ 123

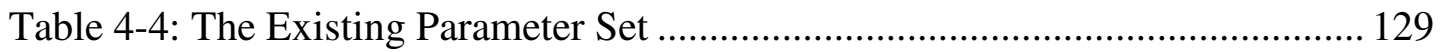

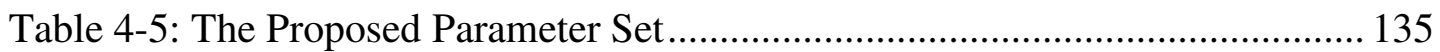




\section{LIST OF FIGURES}

\section{FIGURES}

Fig. 2-1: Structure of an Electrical Power System [1] ….................................... 8

Fig. 2-2: The Time Frame of Basic Power System Dynamic Phenomena [1] ............ 9

Fig. 2-3: Classification of Power System Stability ................................................. 11

Fig. 2-4: Power System Control Structure [2] ….................................................. 13

Fig. 2- 5: The Structure of a Synchronous Generator Unit [1] ................................ 14

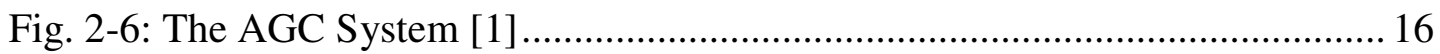

Fig. 2-7: Frequency Control Philosophy as Explained in UCTE Operational

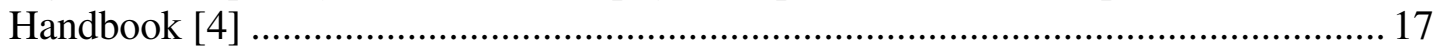

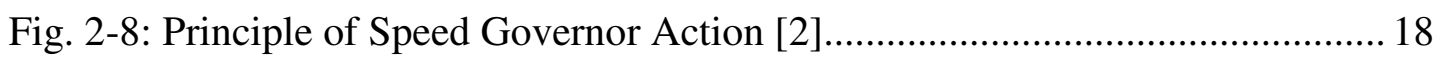

Fig. 2-9: Steady State Power versus Frequency Characteristic of a Governor .......... 19

Fig. 2-10: Frequency of a System with Primary Control Effect after ........................ 20

Fig. 2-11: Contribution of Individual Units to Primary Frequency Control ............... 21

Fig. 2-12: Basic Representation of a Control Area [1] .......................................... 23

Fig. 2-13: Structure of Secondary Controller [1] .................................................. 24

Fig. 2-14: Typical Behavior of System Frequency with Secondary Control [4] ....... 26

Fig. 2-15: Generator (Round Rotor) Connected to an Infinite Bus [1] ...................... 30

Fig. 2-16: Power vs. Load Angle Curves of Unregulated Generator (Dashed Line) and

Fig. 2-17: Power vs. Load Angle Curve Illustrating Transient Stability [20]............ 32

Fig. 2-18: The Nature of Load Angle Oscillations with respect to the Operating Point [1]

Fig. 2-19: The Phasor Representation of the Components of Electrical Power [20]. 37 
Fig. 2 20: Components Determining the Phase Shift Between $\Delta \delta$ and $\Delta \mathrm{Eq}^{\prime}{ }^{\mathrm{EEf}}{ }^{\prime}$

Fig. 2-21: The Phasor Representation of the Effect of $\Delta \delta$ on Damping Torque [1].. 39

Fig. 2-22: The Negative Effect of AVR Action on Damping Torque [1].

Fig. 2- 23: The Phasor Representation of the Negative Effect Introduced by the ..... 41

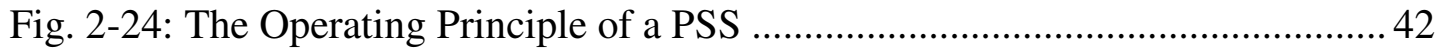

Fig. 2-25: The Phasor Representation of PSS Effect on Damping Torque [20] ........ 43

Fig. 2-26: The Mode Shape of Inter Area Oscillations in

Fig. 2-27: The Frequency Records of Several Areas in case of Inter Area Oscillations $[16]$

Fig. 2-28: The Expected Mode Shape of Inter Area Oscillations in ENTSO-E CESA

System after Synchronous Interconnection of Turkey $(\sim 0.15 \mathrm{~Hz})[16] \ldots \ldots \ldots \ldots \ldots \ldots . . . . .46$

Fig. 2-29: The Expected Undamped Inter Area Oscillations After Interconnected Operation of Turkish Power System ..................................................................... 48

Fig. 2-30: Eigenvalues of the System Related to Rotor Speed ................................ 48

Fig. 2- 31: The Road Map to Preserve Rotor Angle Stability of the Interconnected

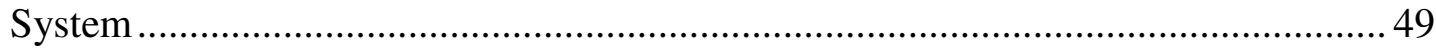

Fig. 3-1: Nonlinear System Configuration with a Dominant Nonlinearity.................60

Fig. 3-2: The Structure of a Dam Type Hydroelectric Power Plant [30]................... 63

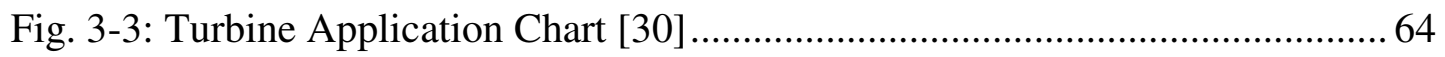

Fig. 3-4: The Structure of a Vertical Generating Unit Equipped with a Francis

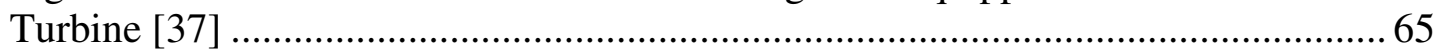

Fig. 3-5: The Most Basic Elements of a Francis Turbine ............................................ 66

Fig. 3-6: A Sample Gate Opening and Effective Flow Area Relationship ................. 68

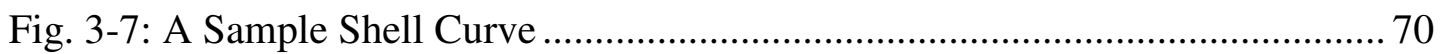

Fig. 3-8: A Sample Graph of Mechanical Power as a Function of Flow and Hydraulic

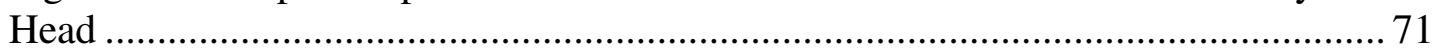

Fig. 3-9: The Schematic of a Hydroelectric Power Plant with a Reservoir ............... 74 
Fig. 3-10: The Combined Turbine and Penstock Dynamical Model

Fig. 3-11: Typical Step Frequency Response of a Hydroelectric Unit

Fig. 3-12: The Block Diagram Representation of a PD governor. 78

Fig. 3-13: The Block Diagram Representation of a PID Governor 79

Fig. 3-14: A Schematic Cross Section of a Generator [1]. 81

Fig. 3-15: The Representation of the Synchronous Generator in dq0 Frame 83

Fig. 3-16: IEEE Type ST1A Excitation System Model [31] .90

Fig. 3-17: The Block Diagram of IEEE Standard PSS2A

Fig. 3-18: The Reduced AVR and PSS Model to be Used in Network Small Signal Analysis [1]

Fig. 4-1: The Graph Comparing the Site Measurements (Red) and Model Output (Blue).

Fig. 4-2: The Graph Comparing the Site Measurements (Red) and Model Output (Blue).

Fig. 4-3: Island Mode Simulation (-5\% Load Change) with the Existing Governor Settings

Fig. 4-4: Island Mode Simulation (+5\% Load Change) with the Existing Governor Settings

Fig. 4-5: $+200 \mathrm{mHz}$ Step Frequency Deviation Simulation with the Existing Governor Settings 108

Fig. 4-6: -200mHz Step Frequency Deviation Simulation with the Existing Governor Settings 109

Fig. 4-7: The Frequency Response of the System with the Existing Governor Settings 111

Fig. 4-8: 0.15 Hz Sinusoidal Frequency Oscillations Simulation 112

Fig. 4- 9: The Phasor Diagram of the Mechanical Power Response of the Unit against 
Fig. 4- 10: The Frequency Response Plots of the Unit with the Existing Governor Settings (Blue) and the Proposed Governor Settings (Red).

Fig. 4-11: 7 Sec Sinusoidal Frequency Oscillations Simulation.

Fig. 4-12: The Phasor Diagram of the Mechanical Power Response of the Unit against Sinusoidal Frequency Input with the Proposed Governor Settings .....

Fig. 4-13: Island Mode Simulation (+5\% Load Change) with the Proposed Governor Settings

Fig. 4-14: Island Mode Simulation (-5\% Load Change) with the Proposed Governor Settings

Fig. 4-15: The Response of Mechanical Power against Step Frequency Deviations

Fig. 4-16: The Response of Mechanical Power against Step Frequency Deviations

Fig. 4-17: The Response of Mechanical Power against Step Frequency Deviations

Fig. 4-18: The Frequency of the Two Interconnected Areas of the Test System after a Transmission Line Outage with the Existing Governor Settings.

Fig. 4-19: The Frequency of the Two Interconnected Areas of the Test System after a Transmission Line Outage with the Proposed Governor Settings

Fig. 4-20: Island Mode Simulation (+5\% Load Change) with the Existing Governor Settings

Fig. 4-21: The Response of Mechanical Power against Step Frequency Deviations

Fig. 4-22: 0.15 Hz Sinusoidal Frequency Oscillations Simulation

Fig. 4- 23: The Phasor Diagram of the Mechanical Power Response of the Unit against

Fig. 4-24: Island Mode Simulation (+5\% Load Change) with the Proposed Governor Settings......

Fig. 4-25: The Response of Mechanical Power against Step Frequency Deviations 
Fig. 4-26: 7 Sec Sinusoidal Frequency Oscillations Simulation

Fig. 4-27: The Phasor Diagram of the Mechanical Power Response of the Unit against Sinusoidal Frequency Input with the Proposed Governor Settings ....

Fig. 4-28: The Frequency of the Two Interconnected Areas of the Test System after a

Transmission Line Outage with the Proposed Governor Settings 142

Fig. 4-29: The Frequency of the Turkish Power System after a 1200MW Outage . 144

Fig. 4-30: The Frequency of the Turkish Power System after a 1200MW Outage. 146 


\section{CHAPTER 1}

\section{INTRODUCTION}

With the recent economical developments throughout the world, the competitive environment in all industries has led to the obligation of increased efficiency. This trend has also significantly affected the electricity industry in a similar manner throughout the past twenty years, by leading to a restructured vertically unbundled system. In this kind of structuring, in order to form a wise and fair competitive environment as the basic requirement of a market, first the financial (trade) organizations (i.e., the retail and the wholesale companies) and the physical organizations (i.e., the generation companies, the transmission companies and the distribution companies) are separated from each other. In a vertically unbundled system structure, it is clear that the transmission companies and the distribution companies are essentially natural monopolies. Hence such companies are regulated organizations, whereas the generation companies, the retail companies, the wholesale companies and the eligible customers are deregulated actors which operate in a deregulated market.

In the vertically unbundled system structure, the main role of the transmission system operator is to "operate" the power system; which means that the transmission operator is responsible from the operation, control, maintenance, expansion and upgrade of the transmission system.

In other words, this role brings the responsibility of real time control and operation of the power system to the transmission system operator together with the other responsibilities. In order to achieve this goal, the transmission system operator requires some ancillary services to be procured as the operator of the balancing and 
settlement market, in order to maintain secure and reliable system operation. The ancillary services defined according to the current directive of the Turkish Power System are:

(i) Primary Frequency Control (i.e., supply and demand balancing)

(ii) Secondary Frequency Control

(iii) Capacity Reserves

(iv) Load Shedding

(v) Reactive Power Control

(vi) System Restoration (i.e., black start)

Although all of the above mentioned ancillary services carry great importance for system operation, this work will focus on the expectations and requirements from the hydroelectric power plants regarding the primary frequency control performance.

In modern practical power systems, the transmission system operator always maintains a primary frequency control reserve capacity at an amount that is sufficient to cover the outage of the biggest generating unit ((n-1) rule), although there are futuristic approaches such as stochastic determination of the amount of the spinning reserve. This operational approach constitutes a necessary capacity for the reliability and security of supply under emergency conditions but an unused capacity under normal system operating conditions, which decreases the total efficiency of the system. In order to overcome this matter of fact, the European Transmission Operators operate as a single synchronous interconnected system such that the (n-1) rule still holds. However, for the entire interconnected region, the total amount of the operational reserve decreases, which increases the efficiency of the transmission system without disturbing the security and reliability of supply. In addition to that, since the mass of the interconnected grid increases, the negative effect of supplydemand imbalances on the system frequency decreases which improves the frequency stability of the system. 
The initial studies regarding the interconnection of the Turkish Power System with the ENTSO-E CESA (Europen Network of Transmission System Operators for Electricity - Continental Europe Synchronous Area) system revealed the feasibility of the project that aims the synchronous interconnected operation of the Turkish Power System and the ENTSO-E CESA (former UCTE) System. The project, which has been led by the Turkish TSO (TEIAS), has been going on for the past ten years and the trial parallel operation of the two systems is scheduled to be in 2010 .

As the first stage of this project, first the detailed survey of the Turkish Power System was completed in order to successfully realize the required analysis regarding the interconnection studies.

In the second phase of the project, the specific problems, which are related to the stability and protection of the Turkish Power System in interconnected operation with the ENTSO-E CESA System, were specified and recommendations regarding feasible solutions to the mentioned problems are made. The results of the second phase of the project have contributed to the recent improvements in the frequency stability of the Turkish Power System.

This study, which is within the scope of the second phase of the interconnection project of the Turkish Power System with the ENTSO-E CESA System, concentrates on the specific problems related to the electromechanical systems of large size hydroelectric power plants regarding low frequency inter area oscillations, which are prone to occur once the interconnected operation of Turkish Power System with the ENTSO-E CESA System is established. Initial simulation studies made within the first phase of the project reveal the expected frequency of inter area oscillations after the interconnected operation as approximately $0.15 \mathrm{~Hz}$, which is in the frequency range of interest of the speed governing structures of turbines, as explained in the first and the second parts of the thesis. Since the dynamical characteristic of the overall system is mainly determined by the major plants and it is infeasible to retune all installed hydroelectric power plants, which constitute $30 \%$ of the total installed power of the Turkish Power System, it is assumed that retuning of governors of 
major hydroelectric power plants will satisfy the objectives regarding the operation of the interconnected system.

In the third chapter of this thesis, the mathematical modeling process of the nonlinear turbine governor model, which is used throughout the governor parameter tuning process, is explained together with the generator and network model used throughout the simulation studies. Since the assumptions made throughout the mathematical modeling study determine the application range and reliability of the simulation model, this part constitutes the most crucial stage of the engineering work performed throughout the study.

In the following part, the governor parameter tuning study with regard to the defined objectives, which consider the contingency handling capability of the control area and the minimization of the negative effects of inter area oscillations on the frequency stability of the system, is explained. Therefore, in this chapter of the thesis, in order to effectively model a hydroelectric unit so as to analyze and determine its behavior in case of dynamically varying system conditions, first the mathematical model of the unit is formed as explained in the third chapter, according to the related dynamical data of the hydroelectric power plants that have been surveyed by both TEIAS and the Power Systems Department of TUBITAK UZAY within the scope of the interconnection project of the Turkish Power System and the ENTSO-E CESA System. The mentioned simulation model of a hydroelectric unit is also verified by site measurements as an integral part of the study.

Afterwards, the expected positive effect of the retuned governor settings of the sample hydroelectric power plants on the frequency stability of the system is shown on a simple multi-machine power system. As a result of those simulations, the retuned governor settings are concluded to be suitable for network operation. Following that, the system wide effect of removing the possible sources of negative damping, which are strongly dependent on the governor settings of the major hydroelectric power plants of the Turkish Power System, is shown. The simulations 
mentioned throughout this chapter are performed by using MATLAB Simulink and DIgSILENT PowerFactory.

In the final chapter, conclusions are made regarding the operation of the hydroelectric power plants considering the frequency stability of the system after synchronous interconnected operation of the Turkish Power System and the ENTSOE CESA System. After explaining the effects of retuned hydroelectric power plants' governor settings on the system damping performance, other counter measures that may have positive effects on damping of low frequency inter area oscillations are introduced as future studies. 


\section{CHAPTER 2}

\section{GENERAL BACKGROUND}

\subsection{Power System Stability}

In engineering, a system is understood to be a set of physical elements acting together and realizing a common goal [1]. The stability of any dynamical system can be defined as the ability of regaining equilibrium state after being subjected to a disturbance.

When assessing power system stability, the main quantities of interest are frequency and voltage. The frequency is a global quantity of the system that is a sign of balance between generation and consumption, whereas voltage is a local quantity that is dependent on the strength of transmission system and consumption at the location of interest. The constancy of those quantities is the sign of stability of a power system.

A power system is nonlinear in nature as any physical system. Similar to any nonlinear system, it is a must to develop the system model to make analysis on the power system. When forming a system model, a commonly used term is the system state which describes the system's operating conditions.

A power system is a highly nonlinear system that is constantly subjected to many random disturbances which can be small or large such as changing demand, short circuits, etc. The stability of the system depends on the initial operating conditions (i.e. state of the system) as well as the nature of disturbance. 
A power system may have many equilibrium points similar to any nonlinear dynamical system. It is evident that the stability of a power system is highly dependent on the equilibrium point prior to the disturbance, i.e., a point that there is no movement in the state trajectory of the system. A stressed, weak system is prone to instability in case of large demand increases or it may show an undesired oscillatory behavior that can be expressed theoretically as the state trajectory of an unstable equilibrium point.

It is evident that power systems are "alive", which means that any power system is subject to continuous disturbances which may be either small or large. However, it is common practice to assume that the system is in steady state, i.e., at the equilibrium point, in system studies.

In practice, any power system can not be designed so as to ensure stability in all possible disturbances due to economical reasons. It is feasible to design and operate a power system so as to ensure stability in most probable contingencies which are generally the loss of highest capacity transmission line or generating unit. This property can also be expressed theoretically as local stability of an equilibrium point. Hence, large-disturbance stability always refers to a specified disturbance scenario. A stable equilibrium set thus has a finite region of attraction; the larger the region, the more robust the system with respect to large disturbances. The region of attraction changes with the operating condition of the power system [7].

Any disturbance in power system results with a new system state affecting many elements. In case of change of frequency or voltage, the regulators in power plants change output quantities to maintain the new system state, also the load characteristics may change with regard to change in voltage or frequency. After the disturbance, the system may preserve stability as a single interconnected network by losing the faulted element or intentional tripping of group of elements by generation dropping or load shedding. Another possibility is that the system may be split into two or more small subsystems operating independently and supplying their own load. 
In any case, the objective is continuity of operation since a complete blackout has many economical and social undesired consequences.

\subsection{Classification of Power System Stability}

A power system is essentially a dynamical system consisting of many individual elements connected together, that is capable of generating, transmitting and distributing electrical energy over a wide geographical area as illustrated in Fig. 2-1. Therefore, a wide variety of dynamic interactions are possible concerning the nature of such a large system. Power system stability is understood as the ability to regain an equilibrium state after being subjected to a physical disturbance [1]. Although the above stability definition applies to any dynamical system, it is also valid for a single element of the system or the system as a whole. For example, a disturbance may cause a generator to lose synchronism without affecting the stability of the remaining system, or another disturbance may cause the instability of the system as a whole.

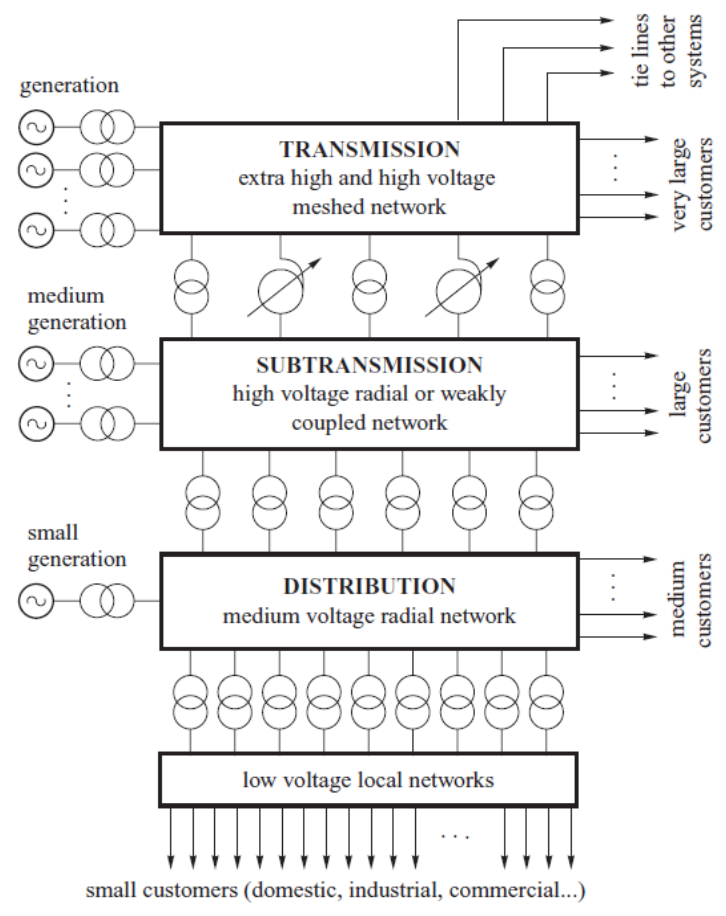

Fig. 2-1: Structure of an Electrical Power System [1] 
Since the power system stability problem concerns a wide variety of elements and causes, it is divided into groups characterized by their cause, consequence time frame, physical character or the place in the system where they occur.

Based on their physical character, the different power system dynamics may be divided into four groups defined as: wave, electromagnetic, electromechanical and thermodynamic [1]. This classification also corresponds to the time frame of interest and is illustrated in Fig. 2-2.

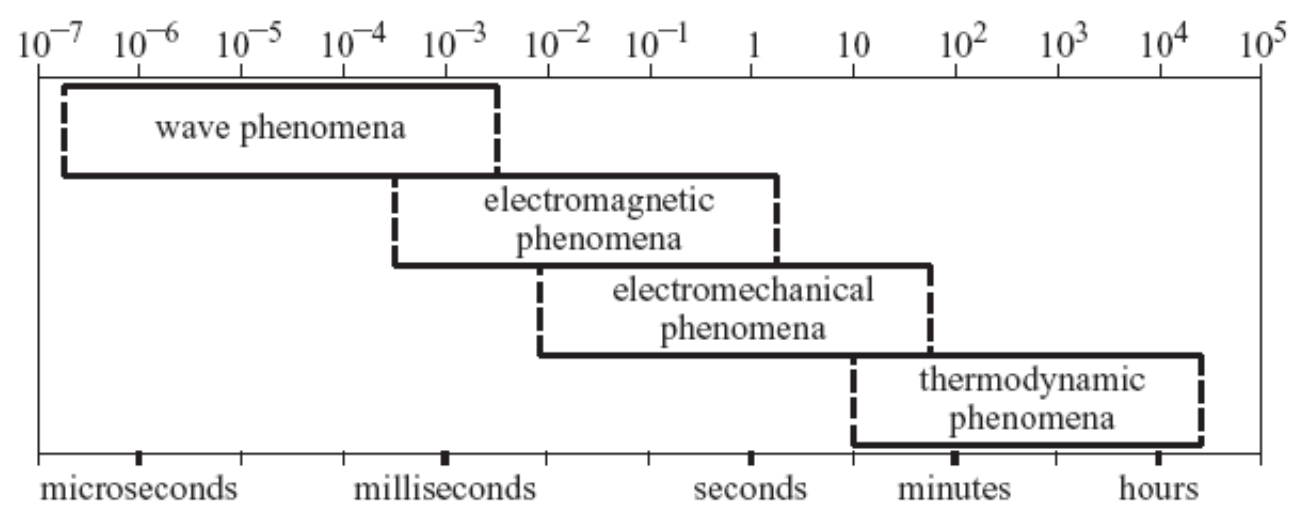

Fig. 2-2: The Time Frame of Basic Power System Dynamic Phenomena [1]

Moving from the left to right along the time scale in Fig. 2-2 corresponds to moving through the power system from the electrical $R L C$ circuits of the transmission network, through the generator armature windings to the field and damper winding, then along the generator rotor to the turbine until finally the boiler is reached.

The first group, namely wave phenomena, concerns the time frame of microseconds, which is caused by lightning surges or line switching actions. As an undesired consequence of this phenomenon, an arc may begin in some equipment, such as transmission lines, and sustain. The outcome of a sustained arc is nothing but a short circuit that can be considered in the time frame of an electromagnetic phenomenon, which eventually triggers isolation of faulted elements from the system. An 
imbalance throughout the system is generally observed with isolation, i.e., outage, of faulted elements, resulting with electromechanical oscillations in the generators. This chain of events generally occupies a time frame of about a minute.

The last group, namely thermodynamic phenomena, is somewhat related to unit commitment problem. Moreover, this phenomenon is also a matter of economy of generation, regarding both the system and market conditions.

As can be seen from the assessment of power system dynamics and their outcomes, a power system is a nonlinear dynamic structure that is subject to many disturbances which sometimes trigger each other. Therefore, the stability problem of such a large system is essentially a single but a big problem that can be analyzed and dealt by being discriminated into smaller parts. From the engineering point of view, the previously mentioned discrimination is nothing but making simplifying assumptions.

Classification of power system stability evidently contributes to power system analysis procedure by identification of effects causing instability and taking precautions against specific problems in the time frame and subject of interest. From the above discussion, it is clear that classification of power system stability problem is essential for practical analysis methodologies.

Three quantities are important for power system operation:

(i) Angles of nodal voltages, also called load angles

(ii) System frequency

(iii) Nodal voltage magnitudes

Hence; power system stability can be divided into three categories as formally illustrated in Fig. 2-3.

(i) Rotor angle stability

(ii) Frequency stability

(iii) Voltage stability 


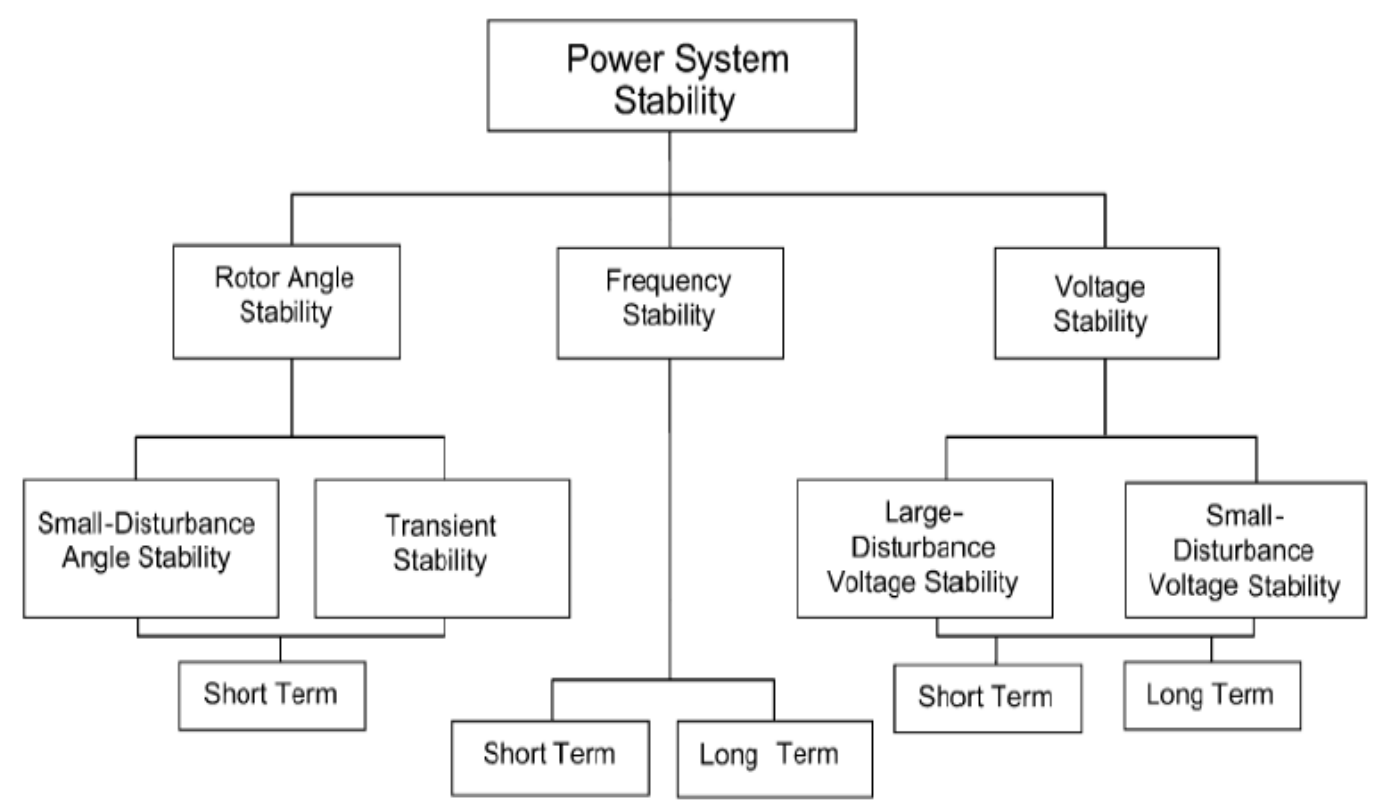

Fig. 2-3: Classification of Power System Stability

As stated before, power systems are nonlinear. Therefore, their stability depends on both the initial conditions and the size of a disturbance. Consequently, angle and voltage stability can be divided into small and large-disturbance, i.e., transient stability. And since modern frequency control action is maintained by both the power plants and the Transmission System Operators (TSOs), the frequency stability problem can be assessed in two groups; namely, short time stability and long term stability.

When a disturbance occurs, firstly, the load flow in the system topology changes that results with voltage change and rotor oscillations in the first few seconds. Afterwards, in case of generation/consumption imbalance, system frequency deviations resulting with mechanical response of power plants. In addition to the previously mentioned chain of events, the oscillations in excitation circuits of synchronous machines caused by voltage regulators also affect the rotor angles of machines, which can also cause instability throughout the system. 
This work mainly focuses on a problem that is specific to a time frame of interest which is both assessed by small disturbance rotor angle stability and short term frequency stability.

\subsection{Power System Control}

As expressed in previous chapters, a power system can be treated as a collection of many nonlinear elements, working together to achieve a main goal. A properly designed and operated power system should meet the following fundamental requirements as discussed in [2]:

(i) The system must always be able to meet the continuously varying load demand both for active power and for reactive power. It is well known that large amount of electrical power can not be stored conveniently, unlike other types of energy. Therefore, sufficient "spinning reserve" of active and reactive power should always be maintained and controlled.

(ii) The system should supply power at minimum possible cost and without significant ecological impact.

(iii) The "quality" of power supply must meet some standards with regard to the constancy of frequency and voltage. Also level of reliability is another matter of interest while assessing power supply quality.

The above explained approach broadly determines the structure of power system controllers. Power system control problem is classified into smaller sub groups as illustrated in Fig. 2-4 to achieve practical and effective solutions. 


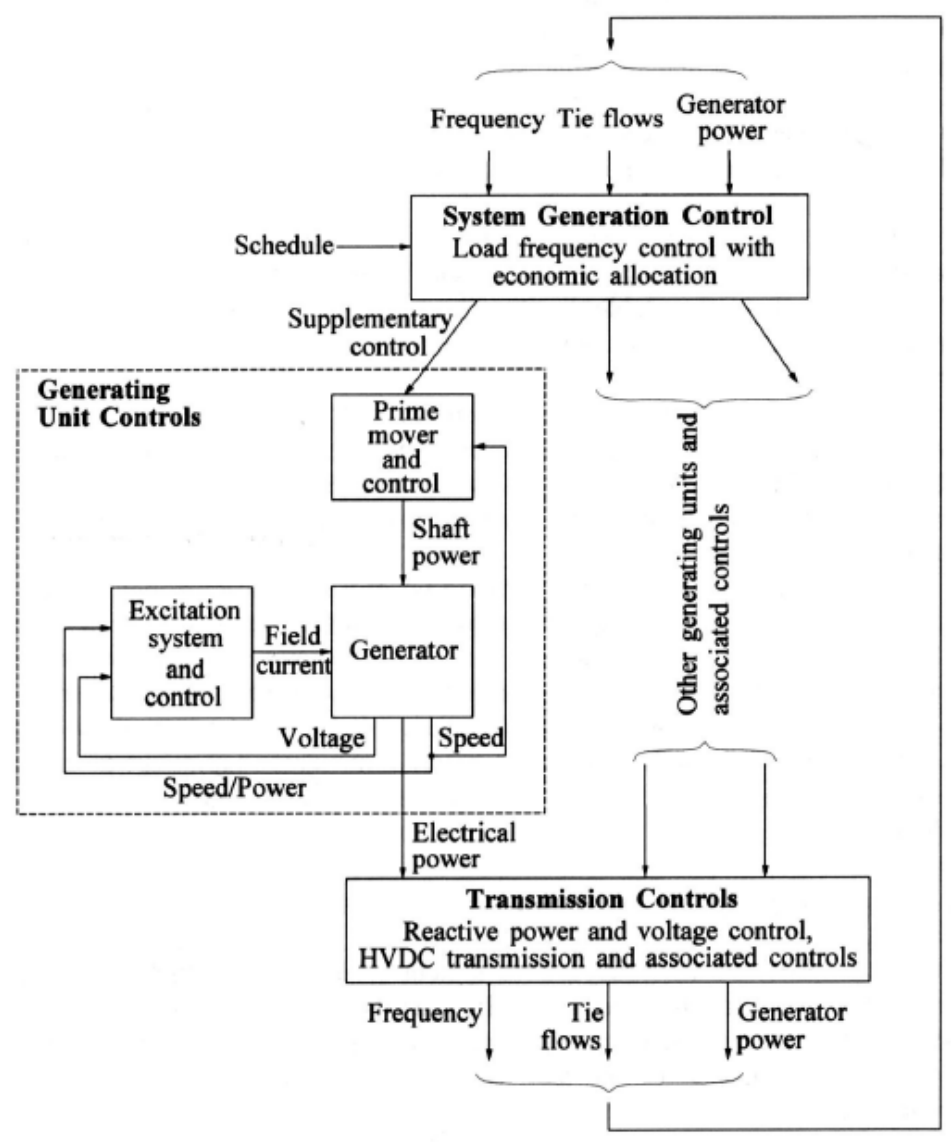

Fig. 2-4: Power System Control Structure [2]

The two basic stages of the approach are listed as:

(i) System level control

(ii) Generation level control

System level control can be treated as a central controller that is responsible from the two variables of interest; namely, voltage and frequency. Transmission utilities, widely known as Transmission System Operators (TSOs) are responsible from governing this level of control after the deregulation and vertical unbundling of the power system. 
In addition to constancy of those two parameters, system level control is also responsible from maintaining the required amount of spinning reserve and continuity of power supply (i.e., satisfying the demand) throughout the whole day, which is treated as unit commitment problem, an output of utility specific electricity market structure.

Generation level control is responsible from maintaining the amount of active power and reactive power to maintain the desired bus voltage at the specific node of interest without disregarding stability issues as illustrated in Fig. 2-5.

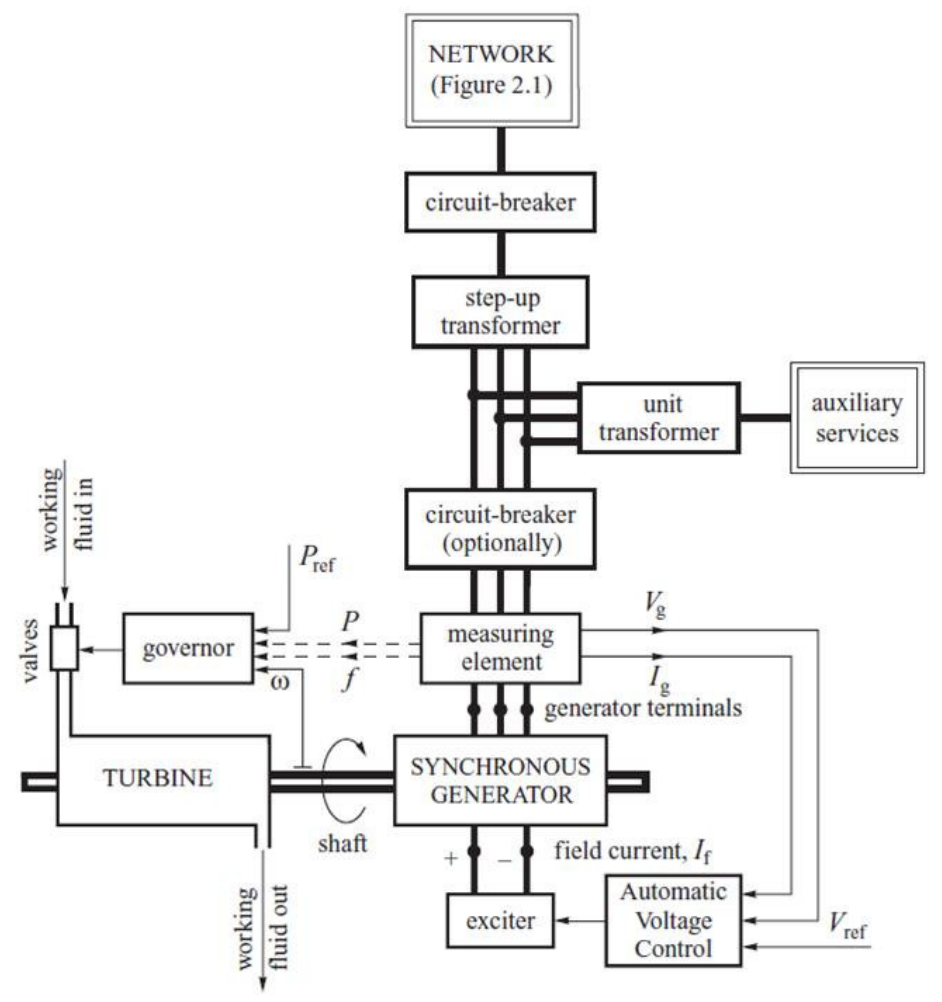

Fig. 2- 5: The Structure of a Synchronous Generator Unit [1] 


\subsubsection{Frequency Control}

In any power system, the total power demand varies continuously throughout the day in a foreseeable manner depending on historical statistical data and also regarding factors such as weather conditions, social factors, etc. In order to better understand the overall control philosophy, it is useful to explain the structure of AGC system which maintains the long term frequency control in large interconnected power systems. The large, slow changes in demand are met centrally by deciding at predefined intervals which generating units will be operating, shut down or in an intermediate hot reserve state. This process of unit commitment may be conducted once per day to give the daily operating schedule, while at shorter intervals, typically every $30 \mathrm{~min}$, economic dispatch determines the actual power output required from each of the committed generators. Smaller, but faster, load changes are dealt with by AGC so as to:

(i) maintain frequency at the scheduled value (frequency control)

(ii) maintain the net power interchanges with neighbouring control areas at their scheduled values (tie-line control)

(iii) maintain power allocation among the units in accordance with area dispatching needs (energy market, security or emergency)

In some systems the role of AGC may be restricted to one or two of the above objectives.

For example, tie-line power control is only used where a number of separate power systems are interconnected and operate under mutually beneficial contractual agreements [1]. 


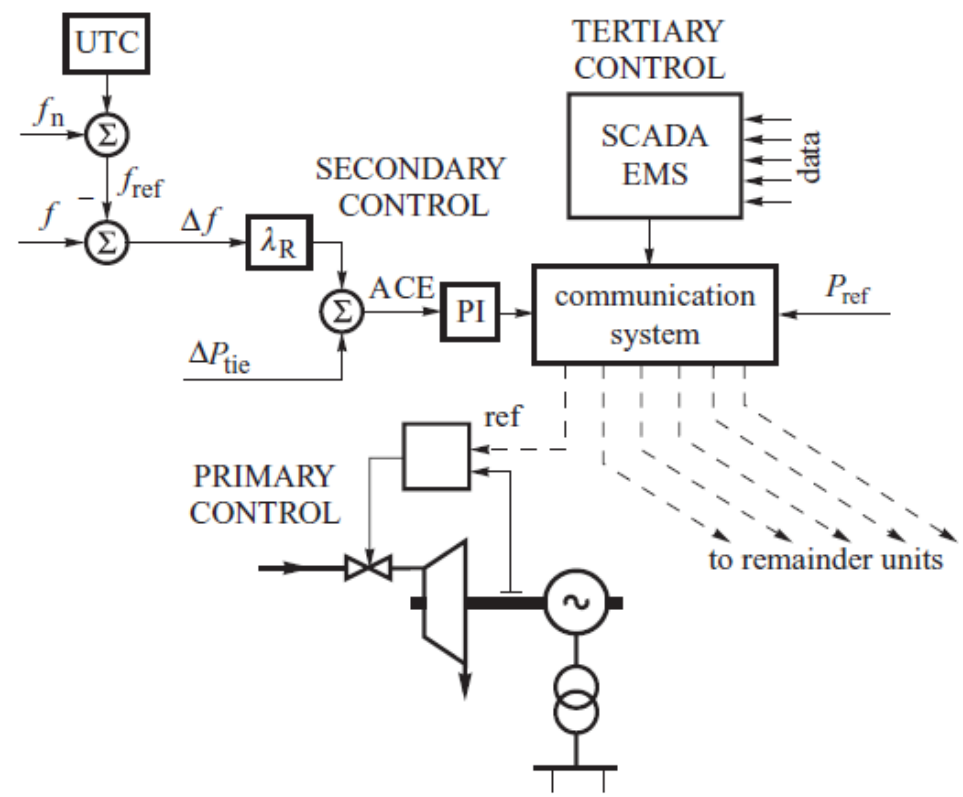

Fig. 2-6: The AGC System [1]

In addition to daily demand variation, power systems are also prone to faults. When a fault occurs in the power system, the generators experience an imbalance between electrical consumption and mechanical generation as a result of the time constants of mechanical quantities which are quite large with respect to electrical quantities. Such disturbances are caused by short circuits in the transmission network and are normally cleared in a few tens of milliseconds, without the need to reduce the generated or consumed power.

However, if a large power mismatch between mechanical power delivered by the turbines and electrical power consumed by the loads occurs in the system, such as connection (or disconnection) of load or disconnection of a generating unit by protection equipment. This imbalance is initially compensated from the kinetic energy of rotating rotors of turbines, generators and motors, which causes them to slow down or accelerate synchronously. Consequently, the frequency in the system will change. This frequency deviation can be divided into a number of stages, allowing the dynamics associated with each of these stages to be assessed separately. 
The electric frequency in the network is a measure for the rotation speed of the synchronized generators. By an increase in the total demand, the system frequency (speed of generators) will decrease and by a decrease in the demand, the system frequency will increase. Regulating units will then perform automatic primary control action via load/frequency control (speed governor) and the balance between demand and generation will be re-established. The frequency deviation is influenced by both the total inertia in the system, and the speed of prime mover. Under undisturbed conditions, the system frequency must be maintained within strict limits in order to ensure the full and rapid deployment of control facilities in response to a disturbance [4].

This part will focus on frequency control in an interconnected power system, using the European ENTSO-E (former UCTE) regulations as the benchmark. It should be noted here that the framework of frequency control in an islanded system, such as in the United Kingdom, may be different from the philosophy explained in this chapter. Also, the meaning of some of the terms may differ from country to country. The demand and generation equilibrium is satisfied by the control philosophy illustrated in Fig. 2-7.

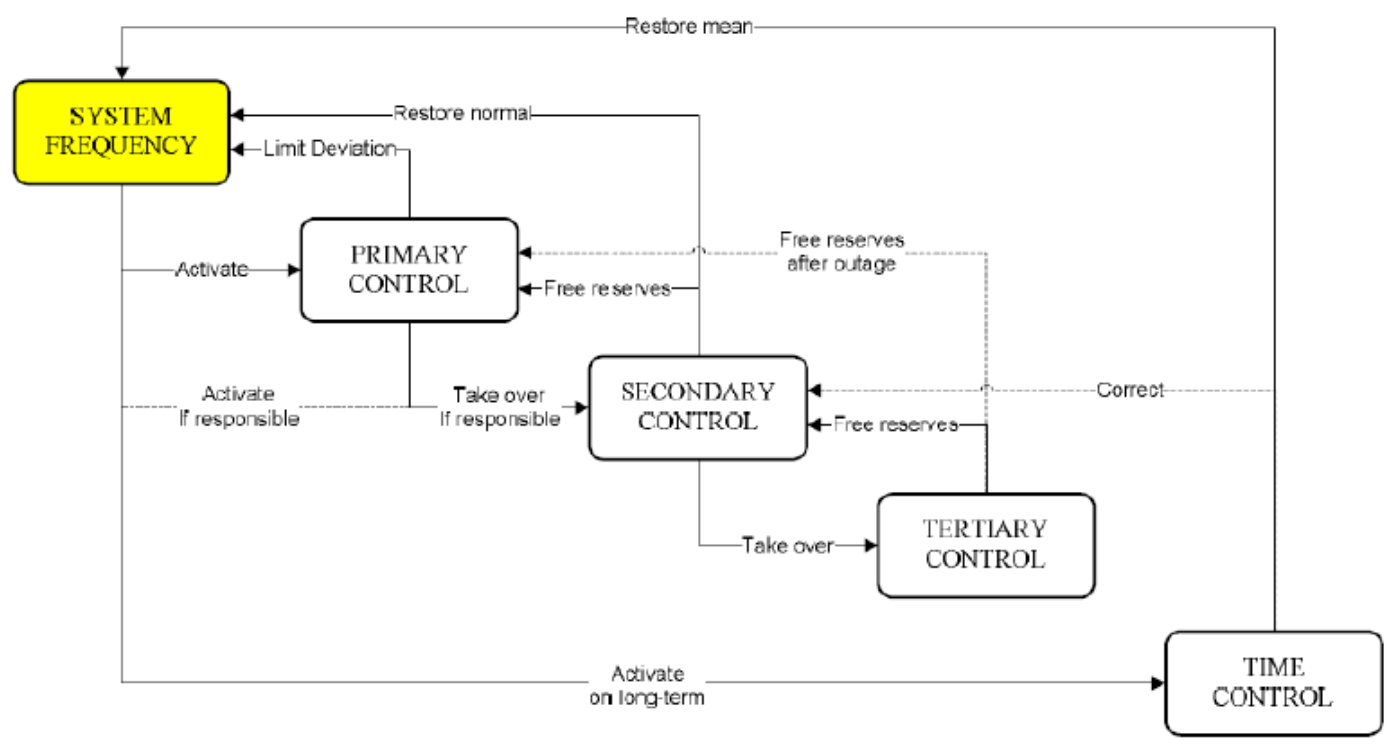

Fig. 2-7: Frequency Control Philosophy as Explained in UCTE Operational Handbook [4] 


\subsubsection{Primary Frequency Control}

In case of a large power mismatch between mechanical power delivered by the turbines and electrical power consumed by the loads occurs in the system, such as connection (or disconnection) of load or disconnection of a generating unit by protection equipment, the difference is compensated from the kinetic energy of rotating rotors of turbines, generators and motors, which causes them to synchronously slow down or accelerate. Consequently, the frequency of the system will change.

Since there are many generators supplying power into the system, some method must be provided to allocate change in active power to the generators. A speed governor on each generating unit provides the primary frequency control function by comparing the measured rotor speed with reference speed and responding in a proportional manner to the difference as illustrated in Fig. 2-8. It is important to note here that some governors operate so as to maintain machine speed constant. Such governors can be used in islanded systems, since a single machine can not change the system frequency.

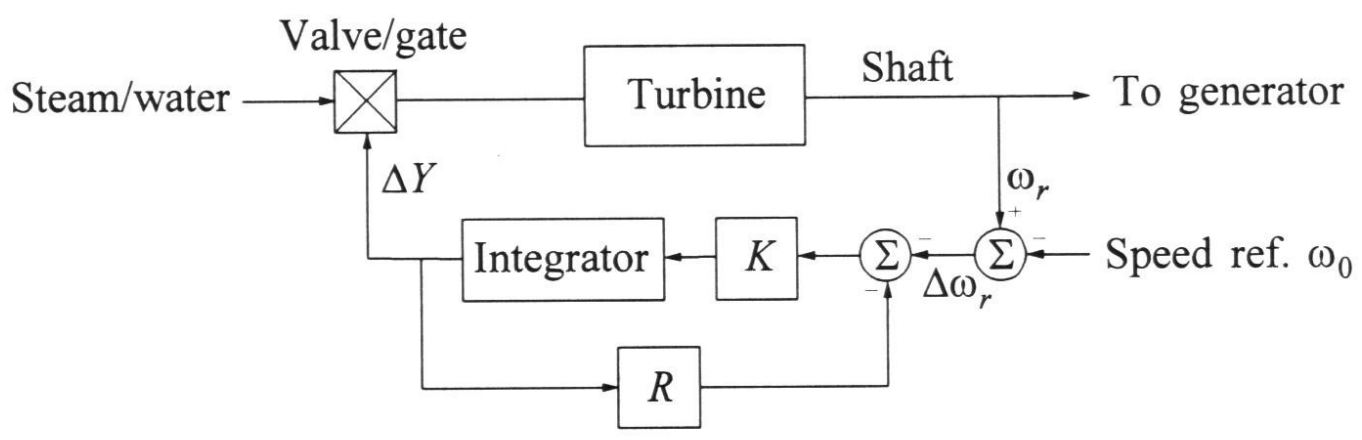

Fig. 2-8: Principle of Speed Governor Action [2]

According to the principle presented in Fig. 2-8, the value of $R$ determines the steady-state speed versus load characteristic of the generating unit. The ratio of speed 
deviation $\left(\Delta w_{r}\right)$ or frequency deviation $(\Delta f)$ to change in valve/gate position $(\Delta Y)$ or power output $(\Delta P)$ is equal to $R$. The parameter $R$ is referred to as speed regulation or droop. It can be expressed in percentage as in (2-1).

$$
R(\%)=\frac{\text { Percentage speed } \text { or frequency change }}{\text { Percentageload change }} \times 100
$$

The graphical representation of this phenomenon is illustrated in Fig. 2-9.

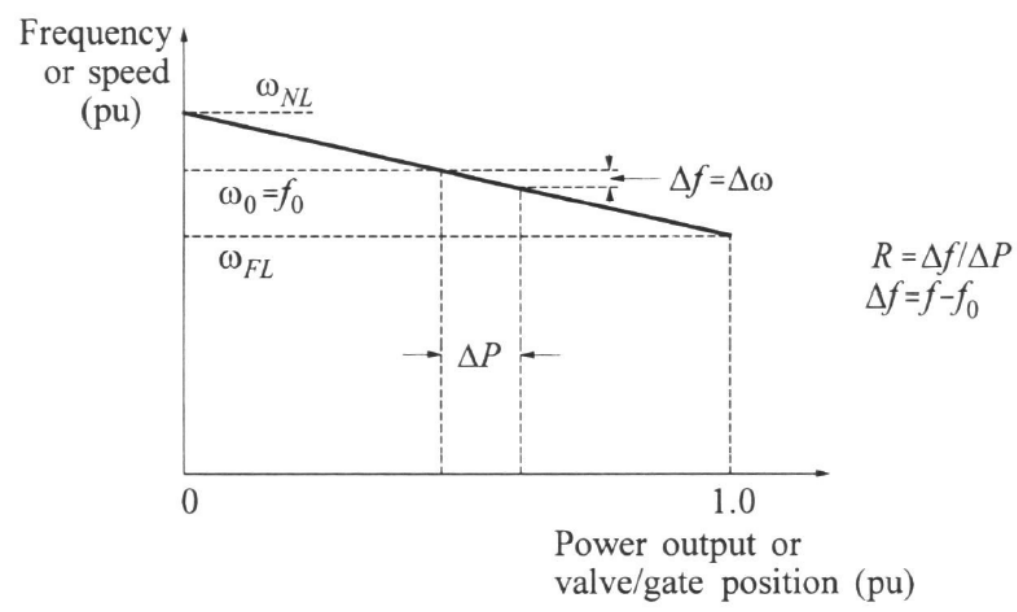

Fig. 2-9: Steady State Power versus Frequency Characteristic of a Governor with Droop Feedback

As stated before, there are many generators connected to a modern interconnected power system. The aim of each independent speed governor is to maintain a mechanical power response from the unit that is proportional to change in grid frequency. Therefore, the main philosophy of primary frequency control may be stated as; maintaining the active power balance between generation and demand, consequently causing the acceleration of the machines to stop which means that the system frequency will deviate from the rated value as illustrated in Fig. 2-10. 


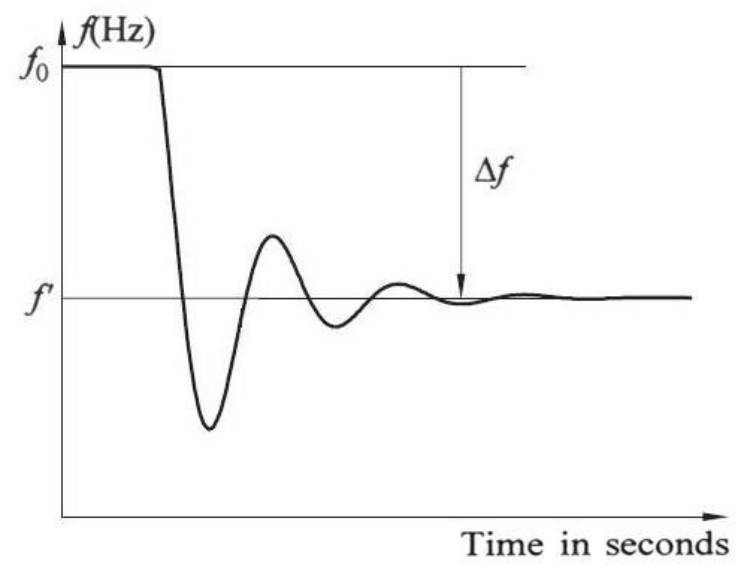

Fig. 2-10: Frequency of a System with Primary Control Effect after a Generation Loss Or Load Increase

All units that are contributing to primary frequency control responds to a frequency deviation such as the one illustrated in Fig. 2-10 in the system according to their speed-droop characteristics as illustrated in Fig. 2-11. The deviation in the system frequency will cause the speed governors of all generators contributing to primary control to respond within a few seconds. The total primary frequency response of the power system will correspond to sum of active power responses of individual units corresponding to the same frequency deviation as expressed by (2-2).

$$
\Delta P_{\text {total }}=\sum_{i=1}^{\substack{\text { number of unitscontributing to } \\ \text { primary frequencycontrol }}} \frac{1}{R_{i}} \Delta f
$$

The controllers increase the mechanical power delivered by the generators until a balance between power output and consumption is re-established. When the balance is re-established, the system frequency stabilizes and remains at a quasi-steady-state value that is different from the nominal frequency as seen in Fig. 2-10, because of the droop of the generators providing proportional type of action. 


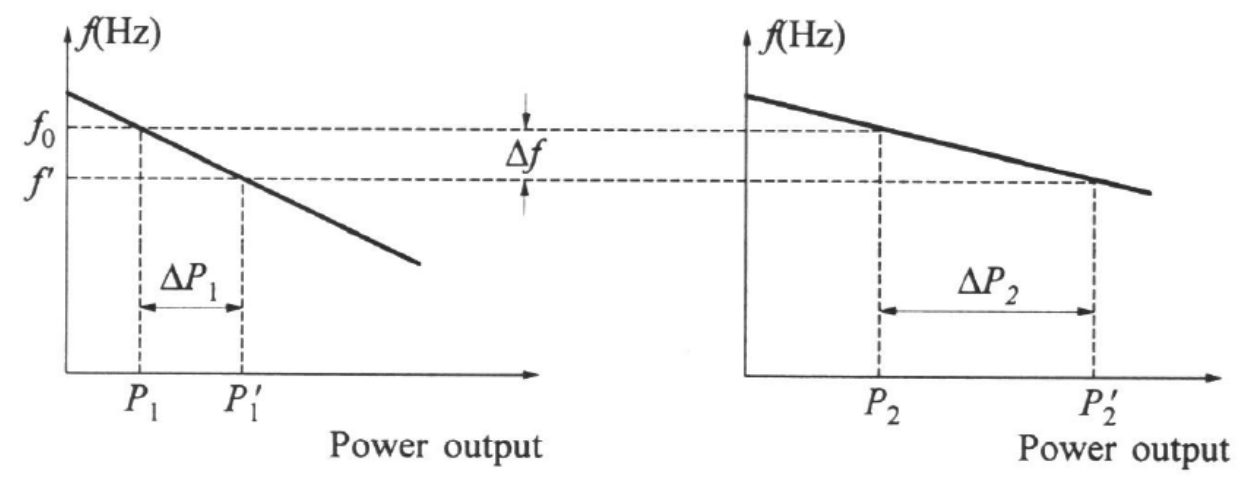

Unit 1

Unit 2

Fig. 2-11: Contribution of Individual Units to Primary Frequency Control

Another important matter of concern about primary frequency control is the amount of reserve. The primary frequency control reserve of a unit is defined as the amount of active power increase or decrease corresponding to $200 \mathrm{mHz}$ frequency deviations. A unit must operate at an output power which is an amount lower than its maximum rating corresponding to the contribution of the unit to a $-200 \mathrm{mHz}$ frequency disturbance and an amount higher than its minimum rating corresponding to the contribution of the unit to a $+200 \mathrm{mHz}$ frequency disturbance in order to effectively participate to primary frequency control. If a turbine is operating at its maximum power limit, then a decrease in the system frequency will not produce a corresponding increase in its power output, similarly if a turbine operates at its minimum power limit then an increase in the system frequency will not produce a corresponding decrease in its power output.

An interconnected system requires coordination. Therefore, the requirements regarding the primary frequency control are normally the subject of agreements between partners cooperating in a given interconnected network. For the European UCTE system, the requirements are defined in the document "UCTE - Ground Rules - Supervision of the application of rules concerning primary and secondary control of frequency and active power in UCTE" [1]. 
For the purposes of primary frequency control, each subsystem in the UCTE system has to ensure a sufficiently large spinning reserve proportional to a given subsystem's share in the overall UCTE energy production. This is referred to as the solidarity principle. It is required that the spinning reserve is uniformly located within each subsystem and the operating points of individual units providing the reserve are such that the whole reserve in the system is activated when the frequency deviation is not more than $200 \mathrm{mHz}$ [1]. The required time of activation of the reserve should not be longer than 30 seconds. To satisfy this condition, the units participating in the primary frequency control should be able to regulate power quickly within allocated percentage of their rated power.

Governors of units that do not participate in the primary frequency control have the first dead zone of the speed - droop characteristic set at $\pm 200 \mathrm{mHz}$, which means that those units do not activate their reserves in case of frequency deviations not exceeding $\pm 200 \mathrm{mHz}$. Those units form an additional primary reserve that activates only for large disturbances which is necessary to defend the system against blackouts.

\subsubsection{Secondary Frequency Control}

Considering the fact that speed governors operate according to the principle as explained in Section 2.3.1.1, then, it is clear that following a disturbance, the system will not be able to return to the nominal frequency on its own, without any additional action. In order to return to the initial frequency, the generation should be intentionally increased by updating active power set points to accelerate or decelerate the units so as to restore the nominal frequency and release the primary frequency control reserve. Such control action on the governing systems of individual turbines is referred to as secondary control.

In interconnected power systems as basically illustrated in Fig. 2-12, whenever an active power imbalance occurs, there may be a risk of unintended power exchanges 
in case of poor secondary control performance. Therefore, in interconnected power systems, each area, or subsystem, has its own central regulator, namely AGC system.

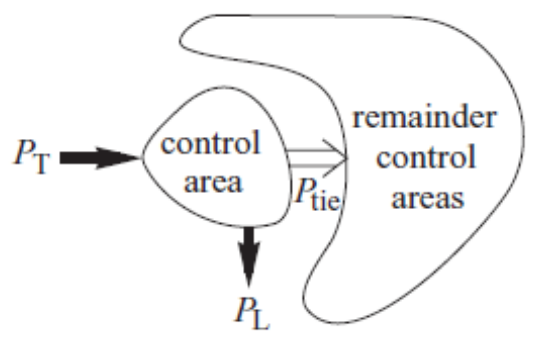

Fig. 2-12: Basic Representation of a Control Area [1]

The power system is in equilibrium if, for each area, the total power generation $\mathrm{P}_{\mathrm{T}}$, the total power demand $\mathrm{P}_{\mathrm{L}}$ and the net tie-line interchange power $\mathrm{P}_{\text {tie }}$ satisfy the condition expressed in (2-3) [1].

$$
P_{T}-\left(P_{L}+P_{t i e}\right)=0
$$

The aim of each area AGC is to maintain nominal frequency (frequency control) and to maintain net tie-line interchanges from the given area at the scheduled values (tie line control). If there is a large disturbance in one subsystem, then AGCs in each area should try to restore the frequency and net tie-line interchanges. This is achieved when the AGC in the area where the imbalance originated enforces an increase in generation equal to the power deficit. In other words, each area regulator should enforce an increased generation covering its own area power imbalance and maintain planned net tie-line interchanges. This is referred to as the non-intervention rule [1]. 


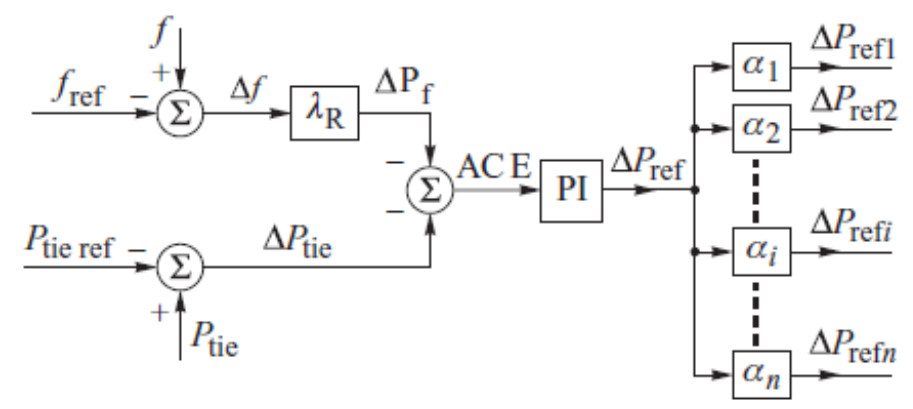

Fig. 2-13: Structure of Secondary Controller [1]

In interconnected systems, the AGCs aim to restore the frequency and maintain tie line flows at the desired amount of exchange. Before forming the area control error, the frequency difference is amplified with a factor called frequency bias $\left(\lambda_{R}\right)$ which represents the change in the generation power which must be enforced to the controlled area of interest in order to compensate for the frequency deviation resulting from the power imbalance in this area.

The value of the bias $\lambda_{R}$ can be assessed according to the frequency stiffness of the power system expressed in $\mathrm{MW} / \mathrm{Hz}$. In practice it is difficult to evaluate the exact value of the stiffness so that imprecise setting of the bias $\lambda_{R}$ in the central regulator may have some undesirable effects on the regulation process.

The exact determination of the actual stiffness in real time is a difficult task as the stiffness is continuously varying due to changes in the demand, its structure and the composition of power plants. In practice, the frequency bias factors $\lambda_{R}$ are set in central regulators in the European UCTE system using a simplified methodology. Each year, the share of a given control area in the total energy production is determined. Then the value of stiffness is estimated for the whole interconnected system. This estimate of stiffness is divided between the control areas in proportion to their annual energy share and that value is set to be the frequency bias factor for each area [1]. 
Area Control Error (ACE) is the sum of both frequency error and tie line flow errors as expressed in (2-4). ACE corresponds to the power by which the total area power generation must be changed in order to maintain both the frequency and the tie-line flows at their scheduled values [1].

$$
A C E=\Delta P_{\text {tie }}-\lambda_{R} \Delta f
$$

By investigating Fig. 2-13, one can easily write

$$
\Delta P_{r e f}=\beta_{R} A C E+\frac{1}{T_{R}} \int_{0}^{t}(A C E) d t
$$

where $\beta_{R}$ and $T_{R}$ are the parameters of the central PI controller.

Secondary frequency control is much slower than the primary frequency control. For the secondary frequency control, activation of the whole range of secondary reserve must be done within 15 min.

The speed of regulation also depends on the type of a unit. Typical values as discussed in [1] can be stated as:

(i) For gas or oil units: $8 \%$ of rated power per minute

(ii) For coal and lignite units: $2-4 \%$ of rated power per minute

(iii) For nuclear units: $1-5 \%$ of rated power per minute

(iv) For hydroelectric units: $30 \%$ of rated power per minute

The sum of regulation ranges for all the generating units active in secondary control is referred to as the bandwidth of secondary control. The positive value of the bandwidth, which is from the maximum to the actual operating point, forms the reserve of secondary control. In the European UCTE system, the required value of the secondary reserve for each control area is in the range of $1 \%$ of the power generated in the area. It is additionally required that the secondary reserve is equal at least to the size of the largest unit operating in the area. This requirement is due to 
the non-intervention rule if the largest unit is suddenly lost. If that happens, secondary control in the area must quickly, in no longer than $15 \mathrm{~min}$, increase the power generated in the area by the value of the lost power [1].

Quality of secondary control is assessed by $\sigma_{90}$ and $\sigma_{99}$ indices which are dependent on standard deviation formulas in daily operation. To assess the quality of secondary control for large disturbances, trumpet curves which are formed by a specific formula regarding the severeness of the disturbance are utilized. The system frequency should stay in an envelope formed by the trumpet curve in case of incidences as illustrated in Fig. 2-14.

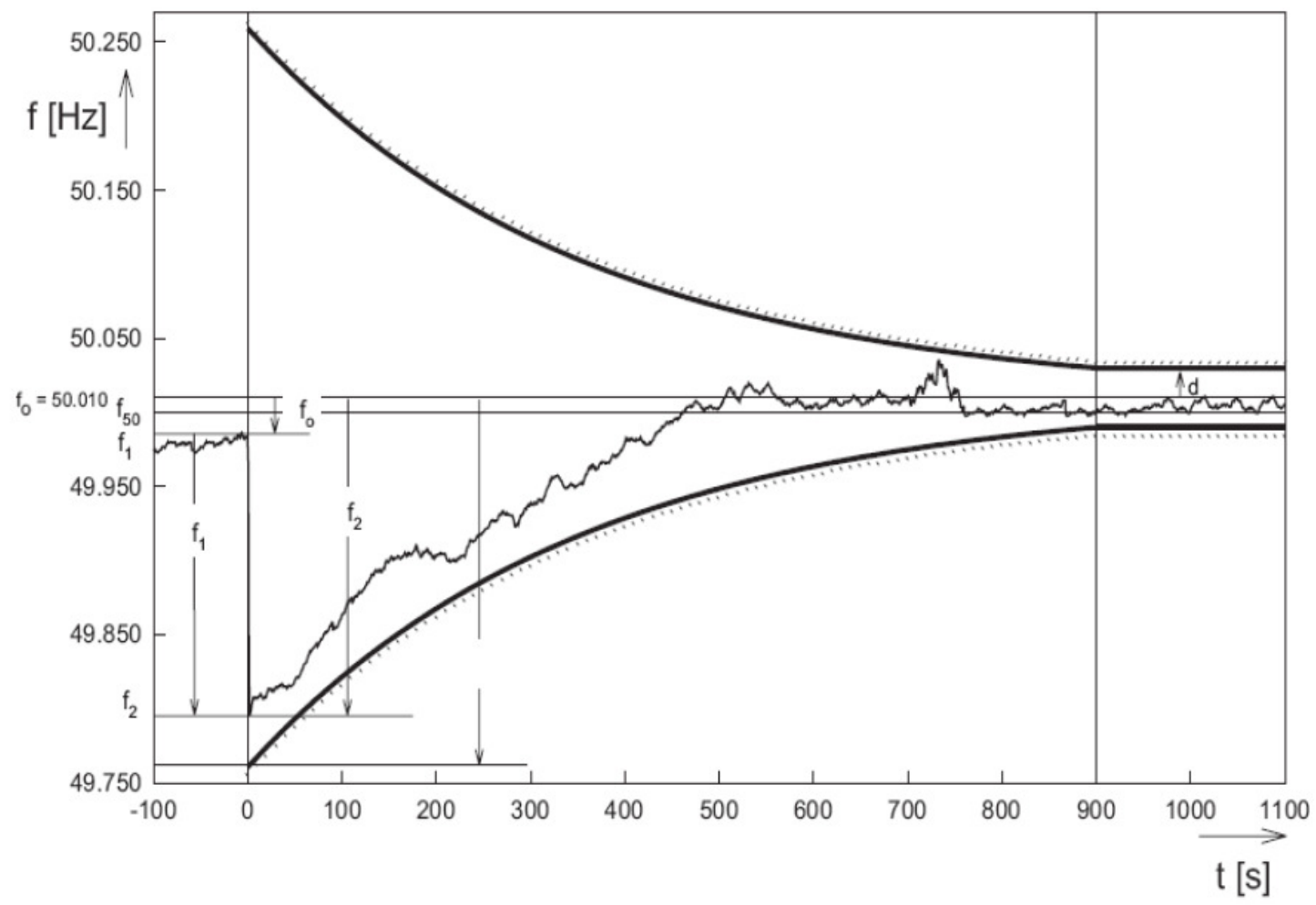

Fig. 2-14: Typical Behavior of System Frequency with Secondary Control [4] 


\subsubsection{Tertiary Frequency Control}

Tertiary control is any automatic or manual change in the working points of generators or loads participating in secondary control, in order to:

(i) guarantee the provision of an adequate secondary control reserve at the right time,

(ii) distribute the secondary control power to the various generators in the best possible way, in terms of economic considerations.

Changes may be achieved by:

(i) connection and tripping of power (gas turbines, reservoir and pumped storage power stations, increasing or reducing the output of generators in service)

(ii) redistributing the output from generators participating in secondary control

(iii) changing the power interchange program between interconnected undertakings

(iv) redistributing the output from generators participating in secondary control

(v) changing the power interchange program between interconnected undertakings

(vi) load control (e.g. centralized telecontrol or controlled load-shedding) [4].

\subsubsection{Time Control}

According to UCTE regulations, the discrepancy between synchronous time and universal coordinated time must not exceed $30 \mathrm{~s}$. The Laufenbourg Control Centre in Switzerland is responsible for the calculation of synchronous time and the organisation of its correction. Correction involves the setting of the set-point 
frequency for secondary control in each area at $49.99 \mathrm{~Hz}$ or $50.01 \mathrm{~Hz}$, depending upon the direction of correction, for periods of one day (from 0 to 24 hours) [4].

\subsubsection{Voltage Control}

Voltage stability is concerned with the ability of a power system to maintain steady voltages at all buses in the system after being subjected to a disturbance from a given initial operating condition. Instability that may result occurs in the form of a progressive fall or rise of voltages of some buses. A possible outcome of voltage instability is loss of load in an area, or tripping of transmission lines and other elements by their protection equipment leading to cascading outages [20]. Similar to frequency control, voltage control can also be assesed as primary voltage control and secondary voltage control.

The primary voltage control is governed by Automatic Voltage Regulators (AVRs) those are equipped in all generators which control the excitation system of the individual generators in such a way to control the voltage at the generator terminal busbar. Primary voltage control is very fast in nature to ensure voltage stability since it is related to electrical quantities.

Primary voltage control will cause the generator voltage to come close to its operating limits since it may cause the machine to operate close to either its under excitation limit which concerns stability of the generator or over excitation limit which considers thermal loading of the rotor circuit. In any case, the AVR of the machine will not be able to react against a consecutive disturbance. Therefore, similar to frequency control philosophy, secondary voltage control is utilized which concerns controlling of voltages at some specific "target" busbars via relatively slow measures such as shunt capacitors or reactors, tap changing transformers or utilization of other generators to support the voltage at the target busbar. Secondary voltage control will result with support of terminal voltage at the problematic generator busbar. This supportive action will end up with an operating point that is 
away from the capability limit at the stressed generator which has responded in a manner to maintain its terminal voltage at the specified set value.

\subsection{Rotor Angle Stability}

As explained in Classification of Power System Stability part, power system stability problem is categorized as:

(i) Frequency stability is concerned with the ability of a power system to maintain steady frequency within a nominal range following a severe system upset resulting in a significant imbalance between generation and load. It depends on the ability to restore balance between system generation and consumption, with minimum loss of load. Instability occurs in the form of sustained frequency swings leading to tripping of generating units and/or loads.

(ii) Voltage stability is concerned with the ability of a power system to maintain steady voltages at all buses in the system after being subjected to a disturbance from a given initial operating condition. Instability occurs in the form of a progressive fall or rise of voltages of some buses. Possible consequences of voltage instability are loss of load in an area, or tripping of transmission lines or other elements by over voltage protection equipment that may cause cascaded problems.

(iii) Rotor angle stability is concerned with the ability of interconnected synchronous machines of a power system to remain in synchronism after being subjected to a disturbance from a given initial operating condition. It depends on the ability to maintain equilibrium between electromagnetic torque and mechanical torque of each synchronous machine in the system. Instability occurs in the form of growing angular swings of some generators leading to their loss of synchronism with other generators.

Since this work focuses on oscillatory stability (i.e., small signal stability), although frequency stability and voltage stability are quite important in the overall power 
system stability problem, rotor angle stability problem will be the main problem assessed in this study. Therefore, frequency and voltage stability topics are explained briefly to a limited extent just for the sake of completeness.

For the stability analysis, a broadly used engineering approach is to assume the generating unit as connected to a very large subsystem, hence an infinite bus of constant frequency and voltage as illustrated in Fig. 2-15.

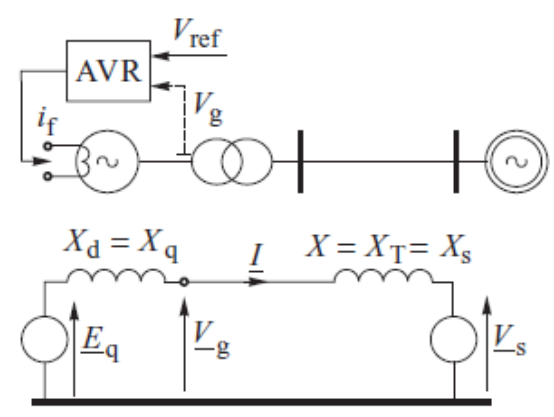

Fig. 2-15: Generator (Round Rotor) Connected to an Infinite Bus [1]

where,

$\mathrm{V}_{\mathrm{s}}$ corresponds to the phasor of the voltage of the infinite bus (the magnitude of which is generally taken as $1 \mathrm{pu}$ and the phase of which is taken as 0 radians) $\mathrm{V}_{\mathrm{g}}$ corresponds to the phasor of the voltage at the generator terminal busbar $\mathrm{E}_{\mathrm{q}}{ }^{\prime}$ corresponds to the phasor of transient internal EMF of the machine I corresponds to the armature current of the machine $\mathrm{X}_{\mathrm{d}}$ corresponds to the $\mathrm{d}$ axis reactance of the machine, which is also referred to as the synchronous reactance of the machine $\mathrm{X}_{\mathrm{T}}$ corresponds to the positive sequence reactance of the unit transformer The power transfer equation of such a basic network is formulated as follows: 


$$
P_{e}=\frac{E_{q}^{\prime} V_{s}}{X_{d}^{\prime}+X_{T}} \sin \delta
$$

This equation is illustrated with the dotted line in Fig. 2-16 as a function of load angle $\delta$, which is the angle difference between the phasors of infinite bus voltage and generator transient internal EMF, namely $\mathrm{E}_{\mathrm{q}}{ }{ }^{[1]}$.

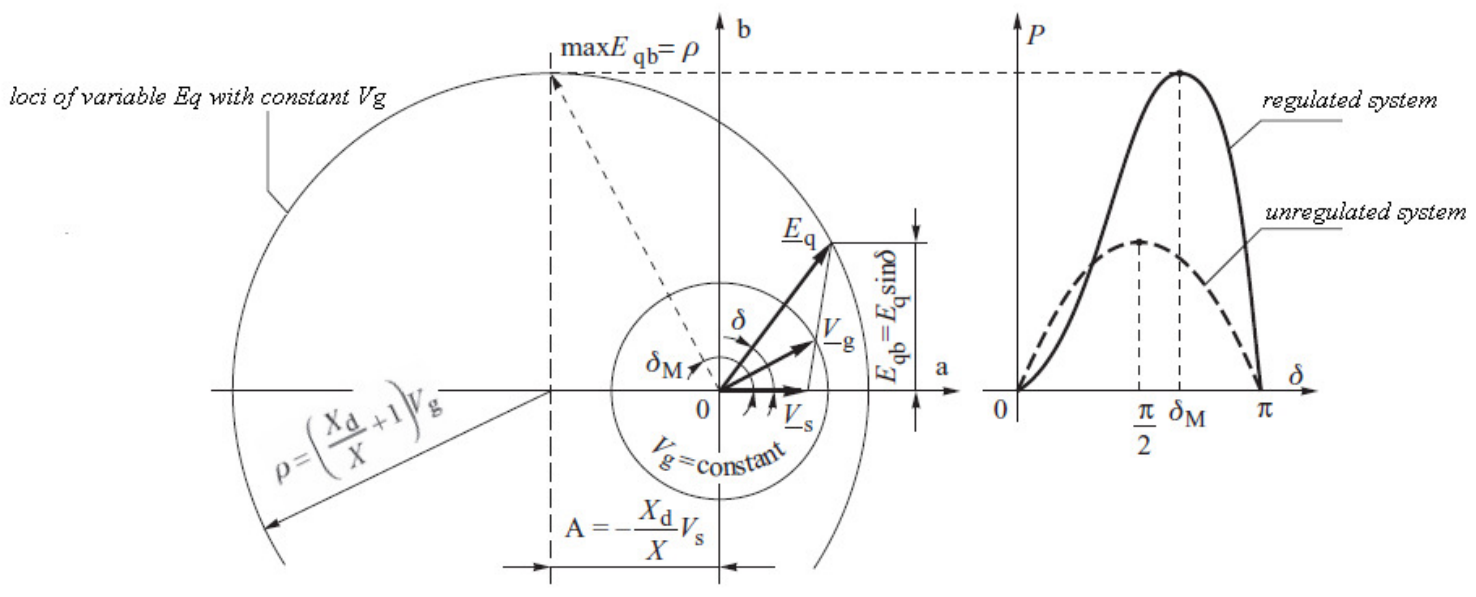

Fig. 2-16: Power vs. Load Angle Curves of Unregulated Generator (Dashed Line) and Regulated Generator (Solid Line) [1]

\subsubsection{Transient Stability}

Transient stability is commonly referred to as "first swing stability". Therefore, as the name implies, transient stability studies concentrate on immediate effect of transmission line disturbances on generator "synchronism". Whenever a fault occurs in the system, the typical behavior of the machine is as illustrated in Fig. 2-17 where $\mathrm{P}_{\mathrm{M}}$ denotes the mechanical power input to the generator by the turbine. 


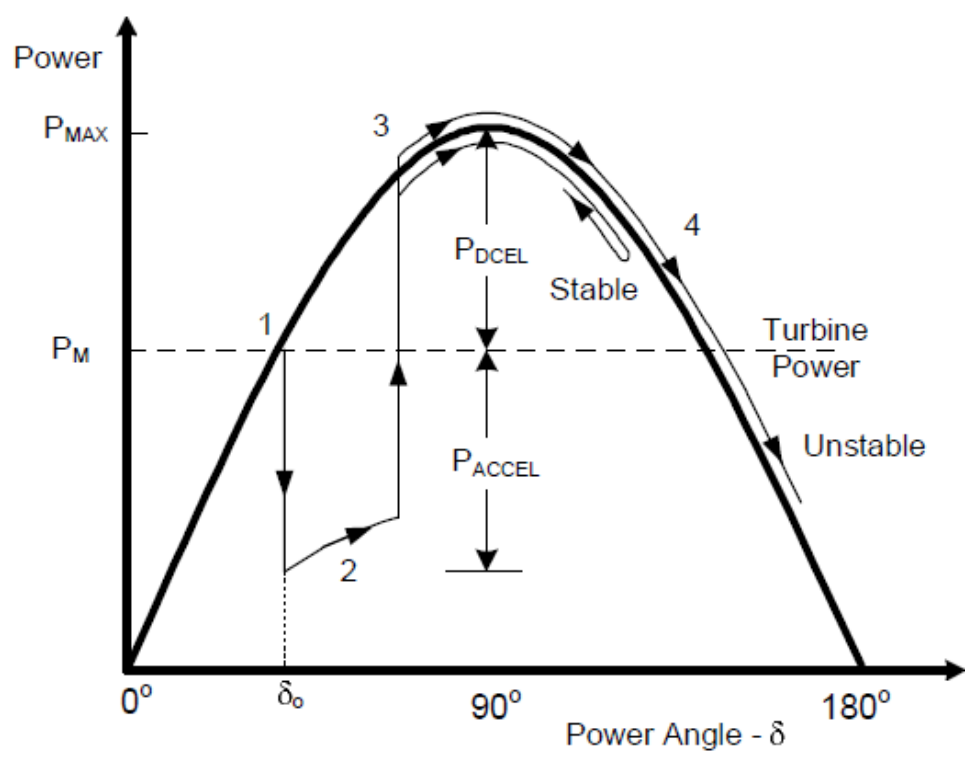

Fig. 2-17: Power vs. Load Angle Curve Illustrating Transient Stability [20]

In order to further assess the phenomenon explained in Fig. 2-17, it is informative to follow the locus illustrated by the solid thin line with arrows. It is assumed that, initially the electrical power and mechanical power of the machine are equal and the load angle is equal to $\delta_{0}$. When a fault in a close location to the generator occurs, the electrical power (i.e. loading) of the machine drastically reduces to the loci numerated by 2 . Since the time frame of interest is the first few electrical swings, the mechanical power of the unit can be assumed to be constant. Therefore, since mechanical power of the unit is greater than electrical power of the machine, the generator rotor will accelerate; hence the load angle will increase until the fault is cleared. When the fault is cleared, assuming the system returns to its initial powerangle characteristics after the fault clearance, electrical power is restored to the corresponding point on the power angle curve (point 3), which is greater than the mechanical power. In this case, the unit will start decelerating (point 4); however since the rotor speed is higher than the grid frequency, the load angle will continue to increase. In case there is enough retarding torque after fault clearing to make up for the acceleration during the fault, the unit will be transiently stable in the first swing and eventually move back to its initial operating point. 
The above explained motion can be mathematically modeled by Newton's second law as (ignoring the machine losses, power in per unit):

$$
P_{m}-P_{e}=2 \frac{H}{w_{s}} \frac{d w}{d t}=2 \frac{H}{w_{s}} \frac{d^{2} \delta}{d t^{2}}
$$

The equation in (2-7) is commonly referred to as "swing equation", where,

$\mathrm{P}_{\mathrm{m}}$ denotes the mechanical power input from the turbine expressed in per unit

$\mathrm{P}_{\mathrm{e}}$ denotes the electrical power of the machine expressed in per unit

$\mathrm{H}$ denotes the inertia constant of the machine (MWsec/MVA)

$\mathrm{w}_{\mathrm{s}}$ denotes the synchronous speed (radians/sec)

w denotes rotor magnetic field frequency (radians/sec)

$\delta$ denotes load angle (radians).

The method used for transient stability assessment is called "equal area criterion", which stems from the fact that the energy that the machine has gained through acceleration should be equal to the energy to be dissipated through deceleration and can be stated as:

$$
\int_{\delta_{0}}^{\delta_{3}}\left(P_{m}-P_{e}\right) d \delta=\int_{\delta_{3}}^{\delta_{4}}\left(P_{e}-P_{m}\right) d \delta
$$

where $\delta_{3}$ is determined by the fault clearing time utilizing the swing equation and the boundary of stability is where $\delta_{4}$ equals to the angle corresponding to $\mathrm{P}_{\mathrm{M}}$, which eventually turns out to be the solution of the power transfer equation considering the second quadrant of the sine function. In this case, $\delta_{3}$ is called the critical clearing angle and the acceleration time corresponding to such an angle change is called critical fault clearing time. It is clear that critical clearing time depends on the initial loading of the machine.

Since the terminal voltage will decrease below the reference value, excitation system forcing to maintain terminal voltage at the reference value during and following the 
fault attempts to increase the electrical power output by raising the generator internal voltage $\mathrm{E}_{\mathrm{q}}$, thus increasing $\mathrm{P}_{\mathrm{MAX}}$. Fast acting excitation systems (i.e., static excitation systems) thus enhance transient stability, although the effect is limited due to the large field inductance of the rotor circuit which prevents sudden increase in $\mathrm{Eq}$ '.

The steady-state stability refers to the ability of a power system to maintain synchronism at all points for incremental slow-moving changes in power output of units or power transmission facilities [20]. Steady-state stability which is a small signal phenomenon is governed by the synchronizing torque similar to transient stability which is a large signal phenomenon. Since a fast acting AVR, as can be readily seen by examination of Fig. 2-16, increases the synchronizing coefficient, it may decrease the damping coefficient due to the mutual coupling of the excitation circuit and the damper circuit. Therefore, it can be stated that a high gain AVR enhances the steady state and transient stability of the machine whereas it reduces the oscillatory stability.

\subsubsection{Oscillatory Stability}

As the short description of transient stability "i.e., first swing stability" implies, a machine being transiently stable on the first swing does not guarantee that it will return to its steady state operating point in a well damped manner and be stable in an oscillatory mode.

After events such as sudden load changes, short circuits, and transmission line switching operations, transient disturbances on machines occur. However, in general, such events give rise to less stable and stressed operating conditions for the grid eventually, which means that the resulting system is in general much weaker than the previous case leading to oscillatory instability.

The most basic solution to improve the dynamic performance of such a weak transmission system is installation of parallel transmission lines, thus lowering the 
equivalent reactance between generation and load. However, such a counter measure is too costly to be implemented; therefore, the damping of the large scale power systems is maintained via control means such as a power system stabilizer (PSS). Utilization and proper implementation of PSSs can significantly improve oscillatory behavior of the system.

\subsubsection{Oscillatory Stability Phenomenon}

As a result of a system disturbance, whether a small disturbance or a large disturbance, a generating unit tends to oscillate around its operating point until it reaches steady state again. The nature of those oscillations is necessarily analogous to the motion of spring mass systems. Such a relationship is expressed by the small signal representation of the "swing equation", which has the same nature as that of spring mass system proposed in the famous paper of De Mello and Concordia [15] as:

$$
2 \frac{H}{w_{s}} \frac{d^{2} \Delta \delta}{d t^{2}}+\frac{D}{w_{s}} \frac{d \Delta \delta}{d t}+K_{1} \Delta \delta+K_{2} \Delta E_{q}^{\prime}=0
$$

where

$\mathrm{H}$ denotes the inertia constant of the machine (MW sec/MVA)

$\mathrm{W}_{\mathrm{s}}$ denotes the synchronous speed (radians/sec)

$\Delta \delta$ denotes small changes in load angle (radians)

D denotes the damping coefficient of the unit (p.u. power change/p.u. speed change)

$\mathrm{K}_{1}$ denotes the synchronizing coefficient of the machine (p.u. $\Delta \mathrm{P} /$ radian)

$\mathrm{K}_{2}$ denotes the effect of transient voltage changes on electrical power (p.u. power change/p.u. internal transient emf change)

$\Delta \mathrm{E}_{\mathrm{q}}$ ' denotes the small changes in generator internal emf behind transient reactance expressed in per unit 
Mathematically speaking, the coefficients $\mathrm{K}_{1}$ and $\mathrm{K}_{2}$ correspond to $\left.\frac{\partial P_{e}}{\partial \delta}\right|_{\delta_{0}}$ and $\left.\frac{\partial P_{e}}{\partial E_{q}^{\prime}}\right|_{E_{q 0}^{\prime}}$ respectively, where $\mathrm{P}_{\mathrm{e}}$ denotes the electrical power, namely the air gap power and they can be obtained by differentiating the electrical power transfer equation. Therefore;

$$
\begin{gathered}
\mathrm{K}_{1}=\left.\frac{\partial P_{E}}{\partial \delta}\right|_{\delta_{0}}=\frac{E_{q}^{\prime} V_{s}}{X_{d}^{\prime}+X_{T}} \cos \delta_{0} \\
\mathrm{~K}_{2}=\left.\frac{\partial P_{E}}{\partial E_{q}^{\prime}}\right|_{E_{q 0}^{\prime}}
\end{gathered}
$$

It is evident from the above equations that, for an unregulated machine, as the initial load angle of the unit increases, the synchronizing coefficient decreases, whereas the damping coefficient increases. Fig. 2-18 illustrates the nature of load angle swings for several operating points.

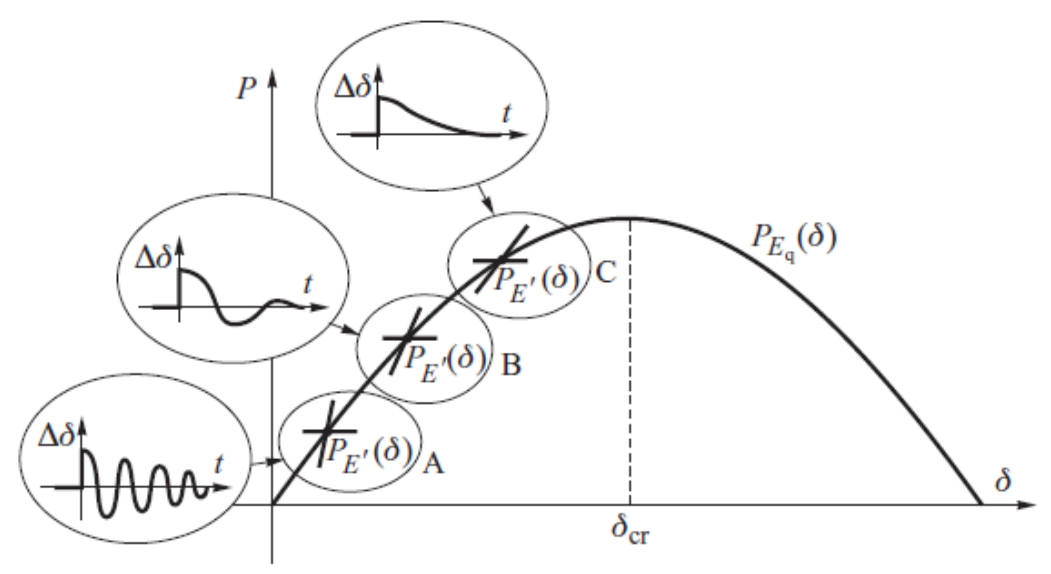

Fig. 2-18: The Nature of Load Angle Oscillations with respect to the Operating Point [1] 
The phasor representation of the synchronous and damping components of electrical power and the resulting unit output power response of the unit to small disturbances is illustrated in Fig. 2-19. As can be seen, the unregulated system has damping power mostly due to the effect of damper windings.

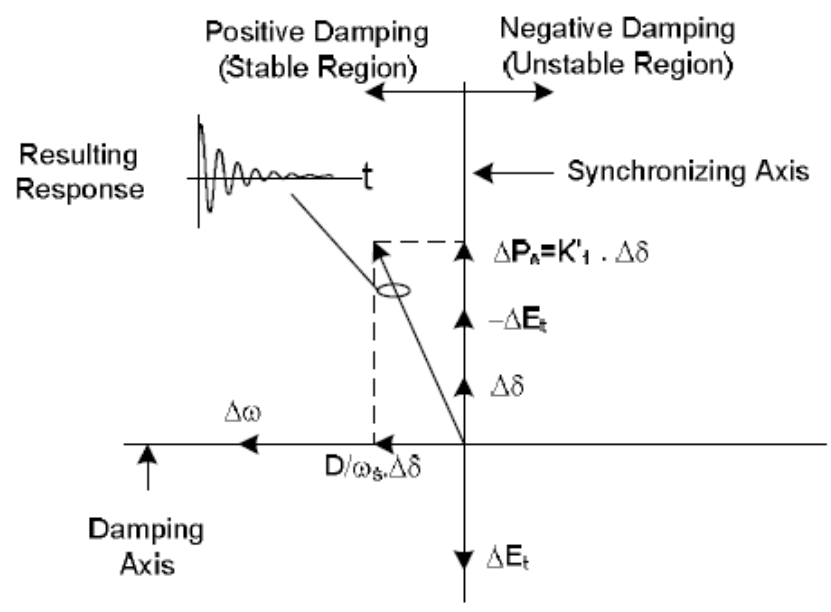

Fig. 2-19: The Phasor Representation of the Components of Electrical Power [20]

\subsubsection{Local Oscillations and Inter Area Oscillations}

There are mainly two distinct types of dynamic oscillations that have introduced major problems on power systems. The first type of oscillations occurs when a generating unit (or group of units) swings with respect to the rest of the system. Such oscillations are called "local oscillations". This kind of oscillations is commonly referred to as "local" since the rest of the power system experiences much less of the oscillation, thus behaving like an infinite bus whereas the specific plant oscillates. Local oscillations tend to occur in case a weak transmission line exists between a generator and the grid, i.e., an isolated power station that transmits power across a single long transmission line. Those modes of oscillation can usually be accurately modeled by the well known single machine and infinite bus model. 
The oscillation frequency of the local modes is characteristically in the 1-2 Hz band, depending mainly on the impedance of the transmission system. Strong transmission systems generally have higher local mode frequencies resulting with less of a tendency toward spontaneous or undamped oscillations.

The second type of oscillations, commonly referred to as "inter area oscillations", are more complex to analyze since combinations of coherent machines on one part of a system swings against machines on another part of the system. Inter area oscillations are mostly related to the undamped or poorly damped modes in the rotor circuits of the machines. The main reasons of inter area modes are the negative effect introduced by fast excitation systems and weak transmission capability of the grid.

\subsubsection{The Effect of Rotor Flux Linkage Variation}

It is evident that the changes in generator internal emf may be both due to load angle

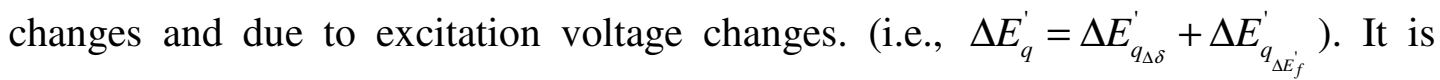
important to note here that both components of $\Delta \mathrm{E}_{\mathrm{q}}$ ' effect the internal emf behind transient reactance via the excitation system and the generator rotor circuit as illustrated in Fig. 2-20. Mathematically speaking;

$$
\Delta E_{q_{\Delta \delta}^{\prime}}^{\prime}=-\frac{A B}{1+B T_{d 0}^{\prime} s} \Delta \delta \text { and } \Delta E_{\Delta E_{f}^{\prime}}^{\prime}=\frac{B}{1+B T_{d 0}^{\prime} s} \Delta E_{f}
$$

where,

$$
A=\left(\frac{1-B}{B}\right) V_{s} \sin \delta_{0} \text { and } B=\frac{\left(X_{d}^{\prime}+X\right)}{\left(X_{d}+X\right)}
$$




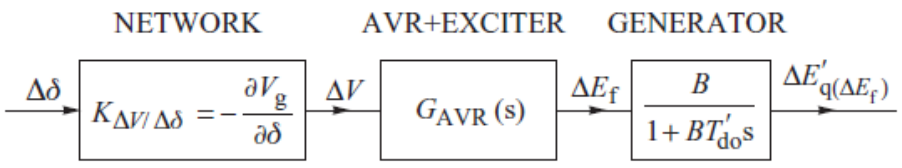

Fig. 2 20: Components Determining the Phase Shift Between $\Delta \delta$ and $\Delta \mathrm{Eq}^{\prime}{ }_{\Delta \mathrm{Ef}}{ }^{\prime}$

Since most of the modern machines are equipped with static exciters, it can be claimed that the transfer function of the AVR introduces a negligible phase lag between $\Delta \mathrm{V}$ and $\Delta \mathrm{E}_{\mathrm{f}}$, hence they may be assumed to be in phase. Therefore, the component of $\mathrm{E}_{\mathrm{q}}{ }^{\prime}$ as effected by $\Delta \delta$ enhances the damping whereas the component $\mathrm{E}_{\mathrm{q}}{ }^{\prime}$ as effected by $\Delta \mathrm{E}_{\mathrm{f}}$ deteriorates damping via the field circuit as illustrated in Fig. $2-21$.

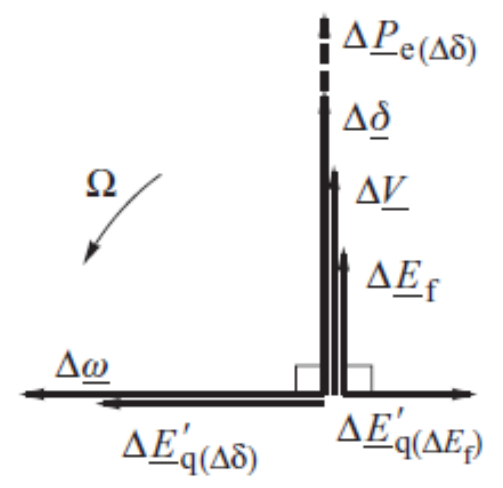

Fig. 2-21: The Phasor Representation of the Effect of $\Delta \delta$ on Damping Torque [1]

\subsubsection{The Effect of AVR on Damper Windings}

As stated before, the main damping torque of the machine is a result of the mutual flux linkage between damper windings and field winding. Similar to a spring mass system, a generator contains inherent damping components that tend to damp rotor oscillations. Therefore, it can be stated that since any system with a dissipative 
element is stable by definition, the natural damping of the system will prevent undamped oscillations unless the damping of the system is deteriorated by external effects.

It has been observed that control actions of voltage regulators and speed governors may constitute sources of negative damping, which can cause undamped electromechanical oscillations. Direct evidence of this has been seen by the fact that sustained oscillations on power systems have been stopped simply by switching voltage regulators from automatic to manual control [20]. However, it is clear that the previously mentioned controllers are crucial for voltage and frequency stability respectively.

The phenomenon of the negative damping introduced by the controllers is illustrated in Fig. 2-22 and Fig. 2-23. As illustrated in Fig. 2-22, the changes in speed create rotating magnetic field hence induced emf on damper windings. Due to the large resistance of damper windings, the induced current in the damper windings lags the induced voltage by an angle less than $\pi / 2$ radians, hence constituting damping torque as well as synchronizing torque. However, the effect of a fast AVR, which slightly lags $\Delta \mathrm{V}$ causes, induced currents in the damper windings via the mutual flux linkage between the field circuit and the damper windings. This component of current induced on damper windings deteriorates the damping torque and may cause instability as illustrated in Fig. 2-23. This component is large enough to cause undamped oscillations when the unit is under stressed conditions that cause large power angles. 

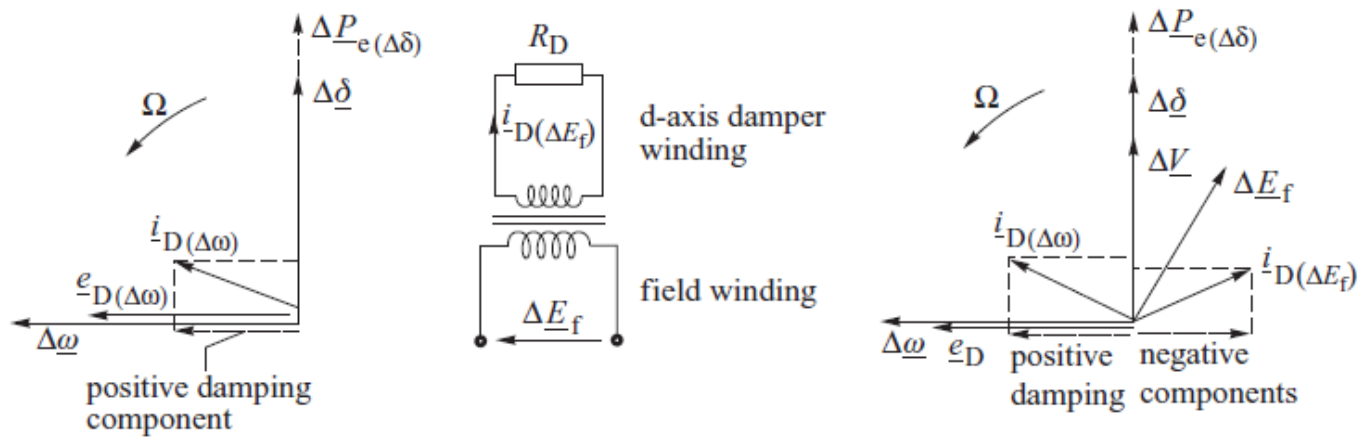

Fig. 2-22: The Negative Effect of AVR Action on Damping Torque [1]

With a slightly lower phase lag in the excitation system, the contribution due to regulator action is more directly in line with the negative damping axis, and has a larger magnitude because of the higher effective gain. The result is more of a tendency toward oscillatory instability with high response and high initial response excitation systems, and generally a greater need for, supplementary stabilizing devices [20].

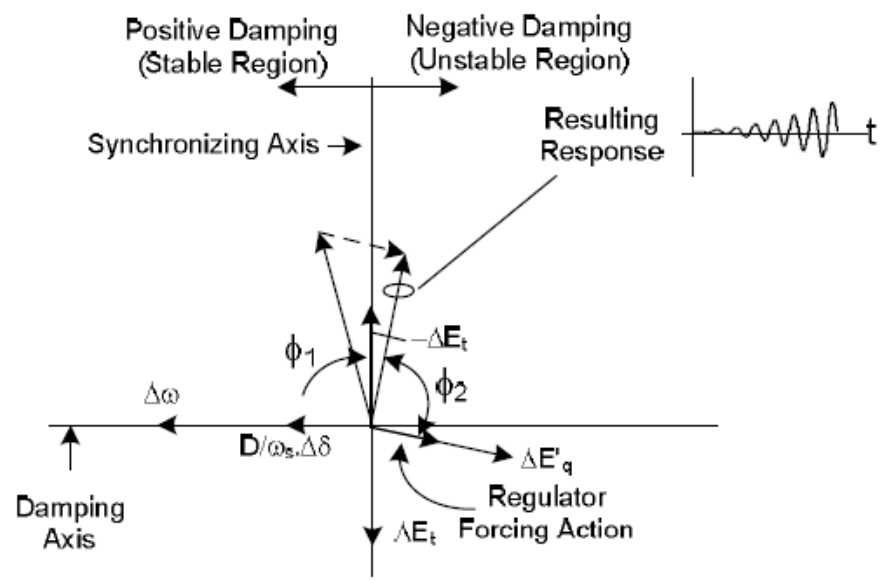

Fig. 2- 23: The Phasor Representation of the Negative Effect Introduced by the Fast Acting AVR [20] 


\subsubsection{Power System Stabilizers}

Since the AVR affects the damping torque via air gap power, it is quite reasonable to cancel this negative effect utilizing supplementary controllers. This supplementary controller is commonly referred to as a power system stabilizer (PSS). PSS is widely used in the United States, Canada and Western Europe. Another solution, preferred in the former Soviet Union, consists of voltage controllers with in-built feedback loops reacting to the time derivative of the voltage error and other quantities [1].

The basic principle of PSS is to introduce a component that is in phase with $\Delta \mathrm{w}$, which is illustrated in Fig. 2-24. To achieve this, a PSS takes feedback from the electrical power, machine speed or both. Afterwards, the signal is filtered to block the low frequency components. Then, the filtered signal is input to the lead lag filter which can be considered as the hearth of the PSS. The signal is filtered so as to maintain enough phase lead to compensate for the lag in the field network. Afterwards, the amplified signal is input to the summing junction in the AVR, which eventually cancels the negative damping component introduced by the high gain AVR.

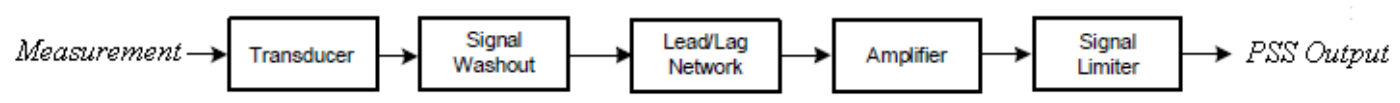

Fig. 2-24: The Operating Principle of a PSS

The phasor representation of the above explained phenomenon is illustrated in Fig. 225. As can be seen, a well tuned PSS cancels the negative effect introduced by the high gain AVR and significantly enhances damping. 


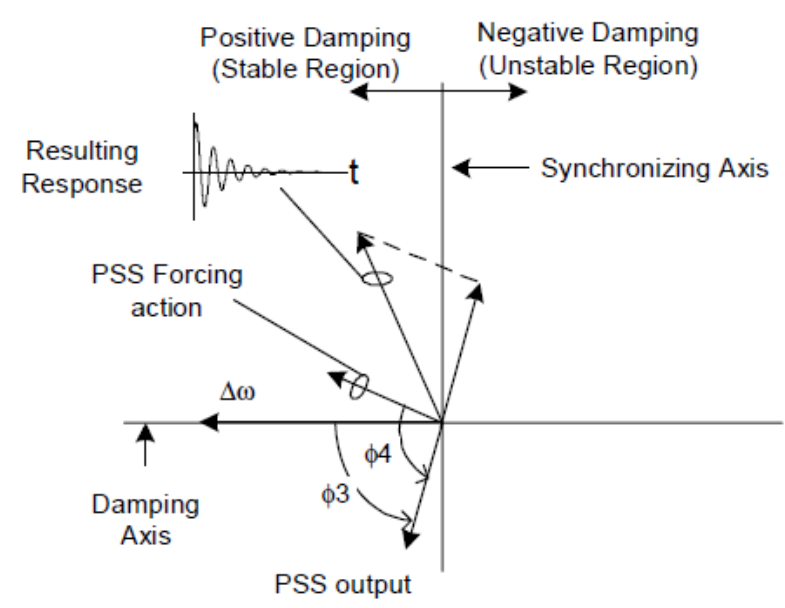

Fig. 2-25: The Phasor Representation of PSS Effect on Damping Torque [20]

\subsection{Problem Definition}

\subsubsection{The Current Practice of Frequency and Voltage Control in Turkish Power System}

At present, the frequency control and voltage control actions in Turkish Power System are being governed according to the Turkish Electricity Market Grid Regulation that has been published on 22/01/2003 which is commonly referred to as the "Grid Code".

According to this regulation, frequency control is performed by primary frequency control (automatically via the speed governors of eligible units), secondary frequency control (via Automatic Generation Control (AGC) System, according to balancing market prices) and tertiary frequency control (manually via instructions given by National Load Dispatch Center (NLDC)) actions.

The requirements regarding participation of generating units to frequency control is defined as follows in the Grid Code: 
- All generation facilities with unit capacities of $50 \mathrm{MW}$ and above or total installed capacity of $100 \mathrm{MW}$ and above except renewable energy resources shall be obligated to participate in primary frequency control; secondary control is also performed via those facilities according to the commercial ancillary services contract. The units with less capacity than the above mentioned can participate in primary and secondary frequency control in case they submit their proposals to TEIAS and the proposal is accepted by TEIAS (Turkish TSO) [19].

According to Grid Code, voltage control is maintained by primary voltage control (automatically via Automatic Voltage Regulators of eligible units), secondary voltage control (manually via instructions given by Regional Load Dispatch Center (RLDC)) actions.

- All generation facilities with unit capacities of $50 \mathrm{MW}$ and above or total installed capacity of $100 \mathrm{MW}$ and above except renewable energy resources should be equipped by Automatic Voltage Regulators.

- The AVRs should be equipped with a PSS, which is tuned so as to enhance damping between the frequency range from $\sim 0 \mathrm{~Hz}$ to $5 \mathrm{~Hz}$ [19].

\subsubsection{The Expected Mode of Inter Area Oscillations after Interconnected Operation and Expected Problems}

Inter area oscillations is an inevitable problem of large scale synchronous interconnected power systems, since utilization of high gain AVRs is inevitable for voltage stability and huge amount of power is transmitted between countries through long transmission lines as a result of electricity trading. The current mode shapes of the inherent inter area oscillations in ENTSO-E CESA (former UCTE) System are illustrated in Fig. 2-26. The length of the vector represents the magnitude, whereas the direction of the vector represents the phase of the rotor angle swing observed in the specified machine. Fig. 2-27 represents a frequency measurement taken from several countries when such inter area swings were observed [16]. 


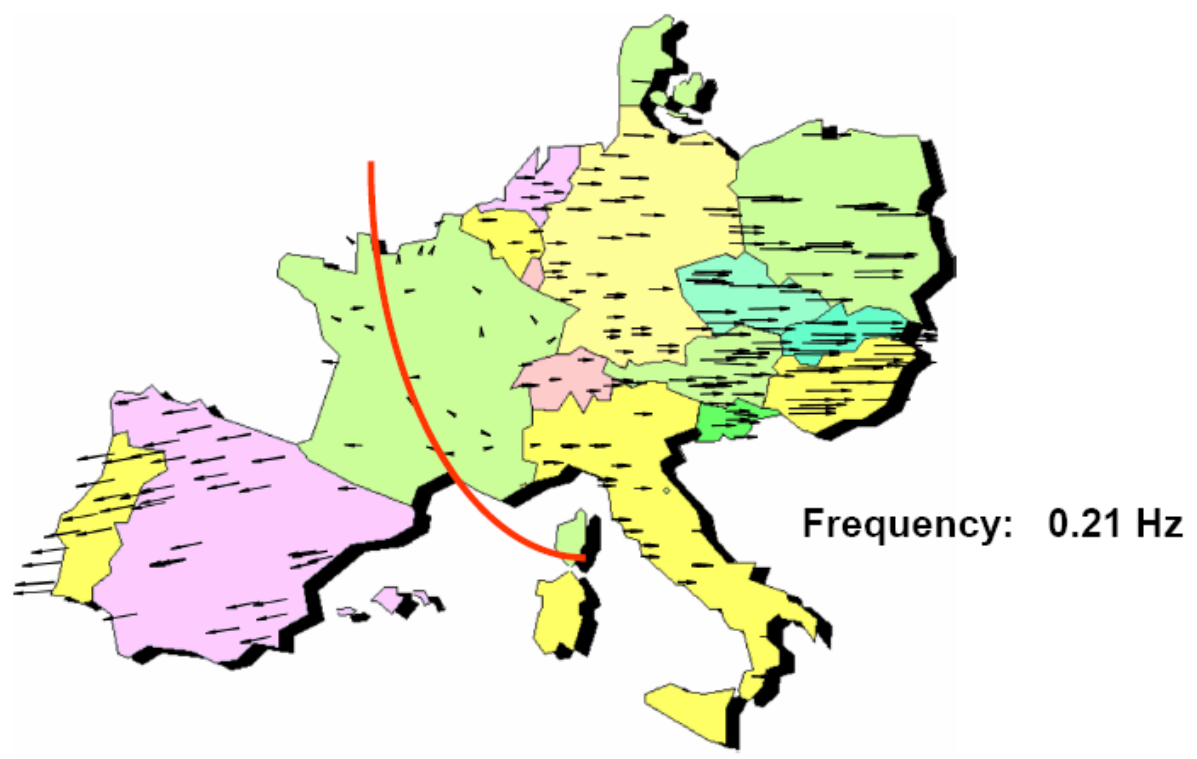

Fig. 2-26: The Mode Shape of Inter Area Oscillations in

Current ENTSO-E CESA System $(\sim 0.2 \mathrm{~Hz})[16]$

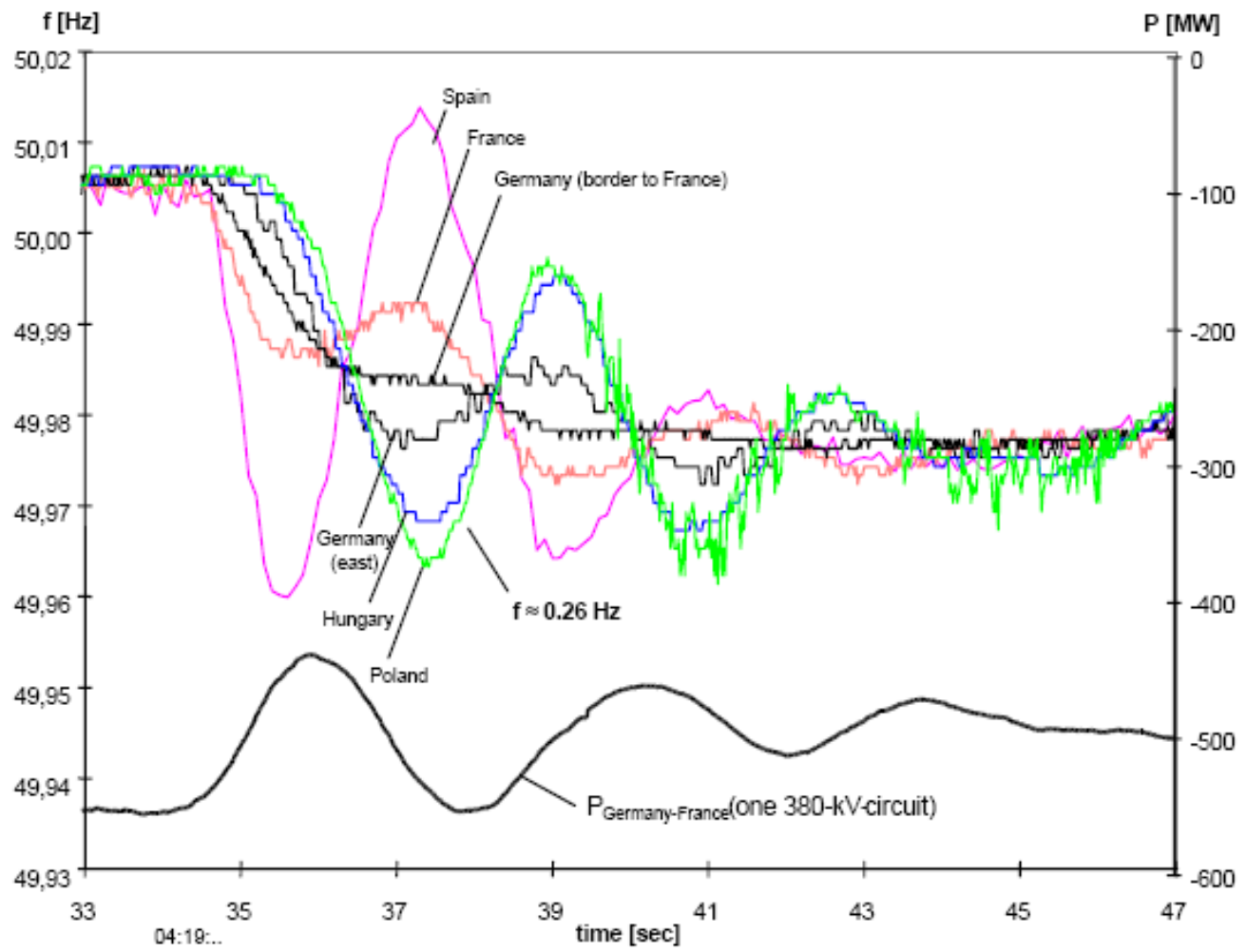

Fig. 2-27: The Frequency Records of Several Areas in case of Inter Area Oscillations [16] 
After the synchronous interconnection of Turkish Power System with ENTSO-E CESA (former UCTE) System, it is expected that inter area oscillations within a frequency neighborhood of $0.15 \mathrm{~Hz}$ (dependent on system conditions) are prone to occur, especially in severe loading scenarios according to the simulation studies. Since the frequency of oscillations is too low, so as to be comparable with load frequency control band, it should be made sure that the contribution of speed governors to that phenomenon is prevented in order to improve, at least not to disturb the damping of the machines, in addition to modified PSS settings, since the expected mode of oscillation is in the control bandwidth of both the PSSs and the governors. The expected mode shape after interconnected operation is illustrated in Fig. 2-28.

Therefore, the Turkish TSO, namely TEIAS, is expected to satisfy the stability criterions for expected inter area modes to guarantee the stable operation of the grid before the interconnection of two systems.

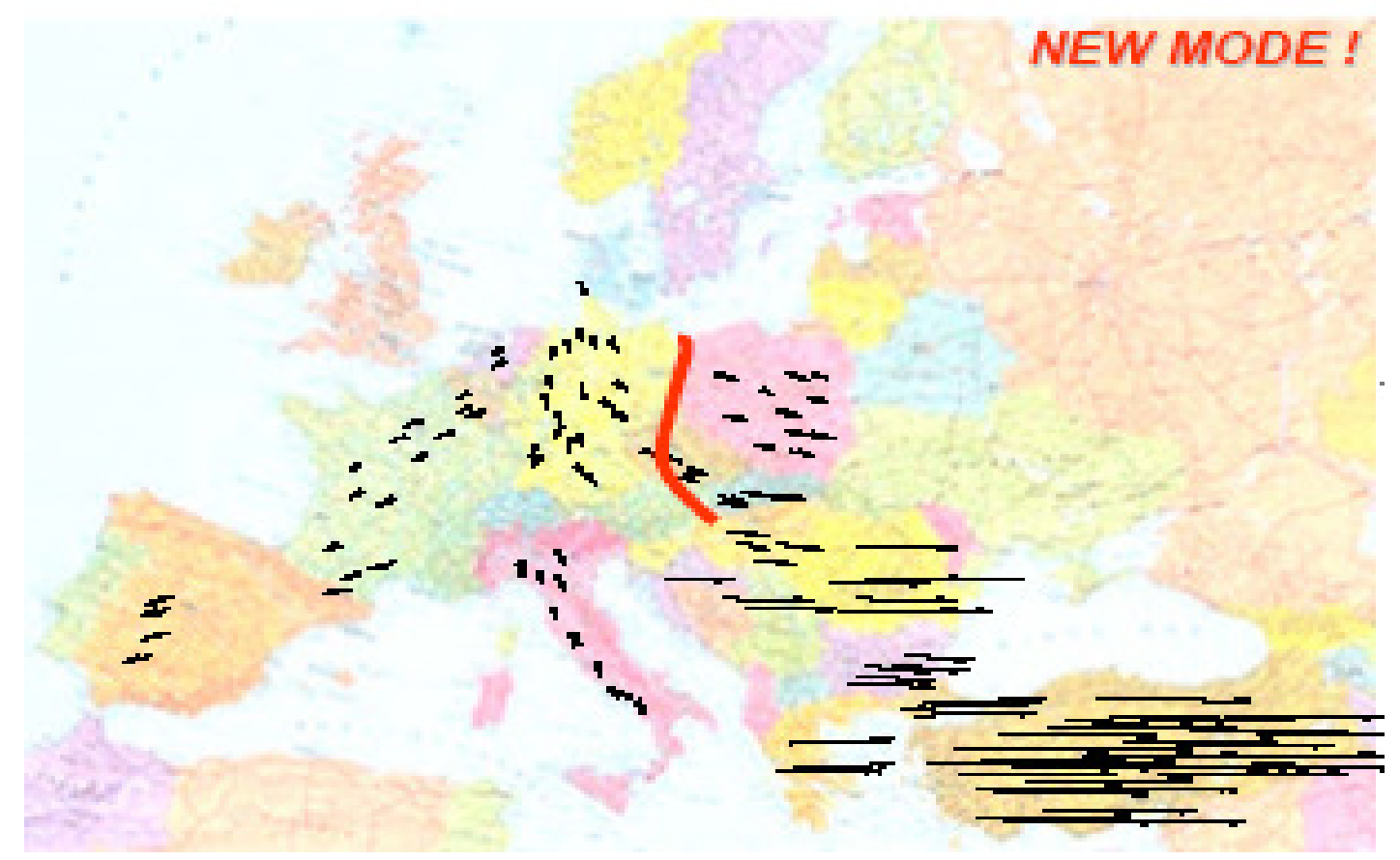

Fig. 2-28: The Expected Mode Shape of Inter Area Oscillations in ENTSO-E CESA System after Synchronous Interconnection of Turkey $(\sim 0.15 \mathrm{~Hz})[16]$ 
As a part of the "Rehabilitation of Frequency Control Performance of Turkish Power System" project, in order to investigate the dynamical behavior of the interconnected grid, nonlinear dynamic simulations have been performed by using the ENTSO-E CESA System and Turkish Power System dynamical data provided by the University of Rostock, the Power Systems Department of TUBITAK UZAY and partially by TEIAS, respectively. Fig. 2-29 illustrates the simulated frequencies after an event, at several busbars of Turkish Power System, in case the two power systems are interconnected without taking any precautions. Eigenvalue (linear) analysis is also performed on the previously mentioned model and the resultant pole-zero map related to eigenvalues of the system related to rotor speed is illustrated in Fig. 2-30, in complex domain. It can be seen that there exists an undamped mode at a frequency of $\sim 0.13 \mathrm{~Hz}$ in the linearized model, which causes expected undamped growing oscillations. This expectation is also verified by dynamical time domain simulation behavior of the nonlinear system model as illustrated in Fig. 2-30. The expected most poorly damped modes are tabulated in Table 2-1.

Table 2-1: The List of Most Poorly Damped Modes Expected after Interconnected Operation

\begin{tabular}{|c|c|c|c|c|c|c|}
\hline & Real Part & $\begin{array}{c}\text { Imaginary } \\
\text { Part }\end{array}$ & Magnitude & $\begin{array}{c}\text { Angle } \\
\text { (Degrees) }\end{array}$ & $\begin{array}{c}\text { Damped } \\
\text { Frequency }(\mathrm{Hz})\end{array}$ & $\begin{array}{c}\text { Damping } \\
\text { Ratio }\end{array}$ \\
\hline Mode 1 & -0.08605422 & 0.8066736 & 0.8112507 & 96.09 & 0.1283861 & 0.106076 \\
\hline Mode 2 & 0.2566212 & 0.7696678 & 0.8113217 & 71.56 & 0.1224964 & -0.3163002 \\
\hline Mode 3 & -0.2184997 & 0.7398701 & 0.7714596 & 106.453 & 0.117754 & 0.283229 \\
\hline Mode 4 & -0.3210125 & 0.7597677 & 0.8248006 & 112.91 & 0.1209208 & 0.3892001 \\
\hline Mode 5 & -0.2 & 0.6 & 0.6324555 & 108.44 & 0.09549297 & 0.3162278 \\
\hline Mode 6 & -0.2490904 & 0.6250772 & 0.6728801 & 111.73 & 0.09948413 & 0.3701855 \\
\hline Mode 7 & -0.2484702 & 0.6244376 & 0.6720563 & 111.70 & 0.09938233 & 0.3697163 \\
\hline
\end{tabular}




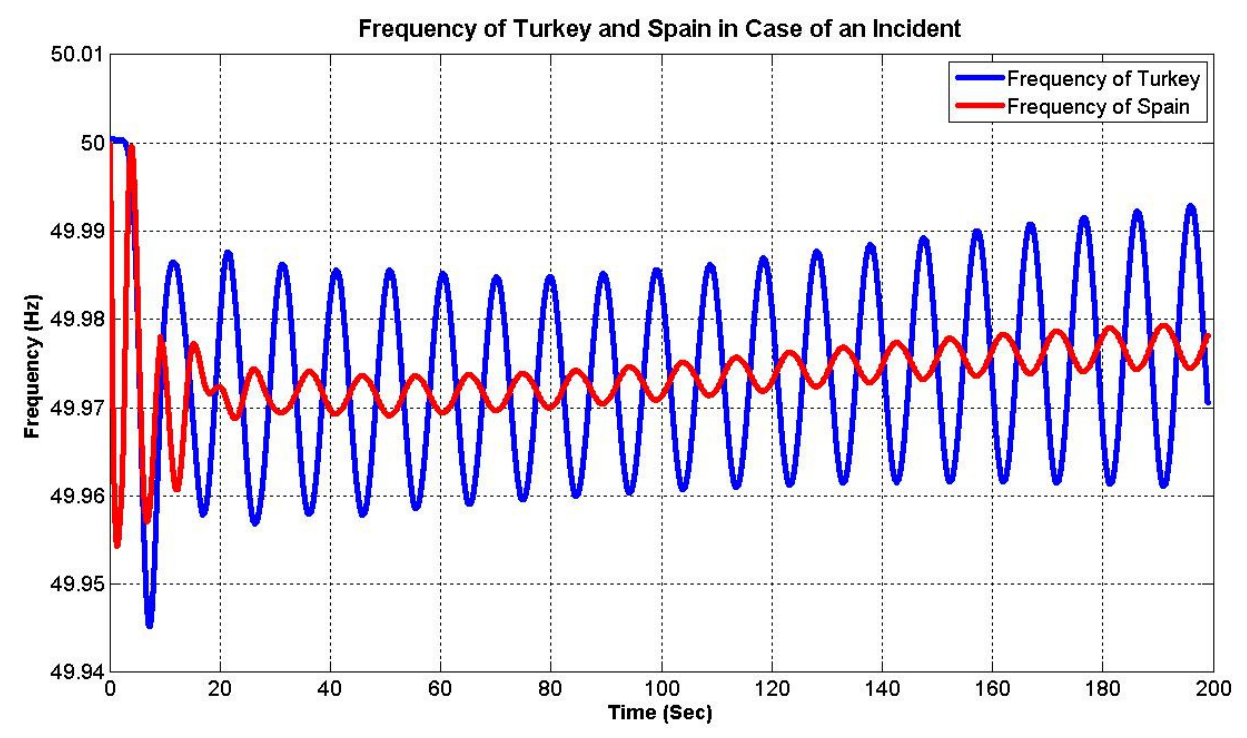

Fig. 2-29: The Expected Undamped Inter Area Oscillations After Interconnected Operation of Turkish Power System

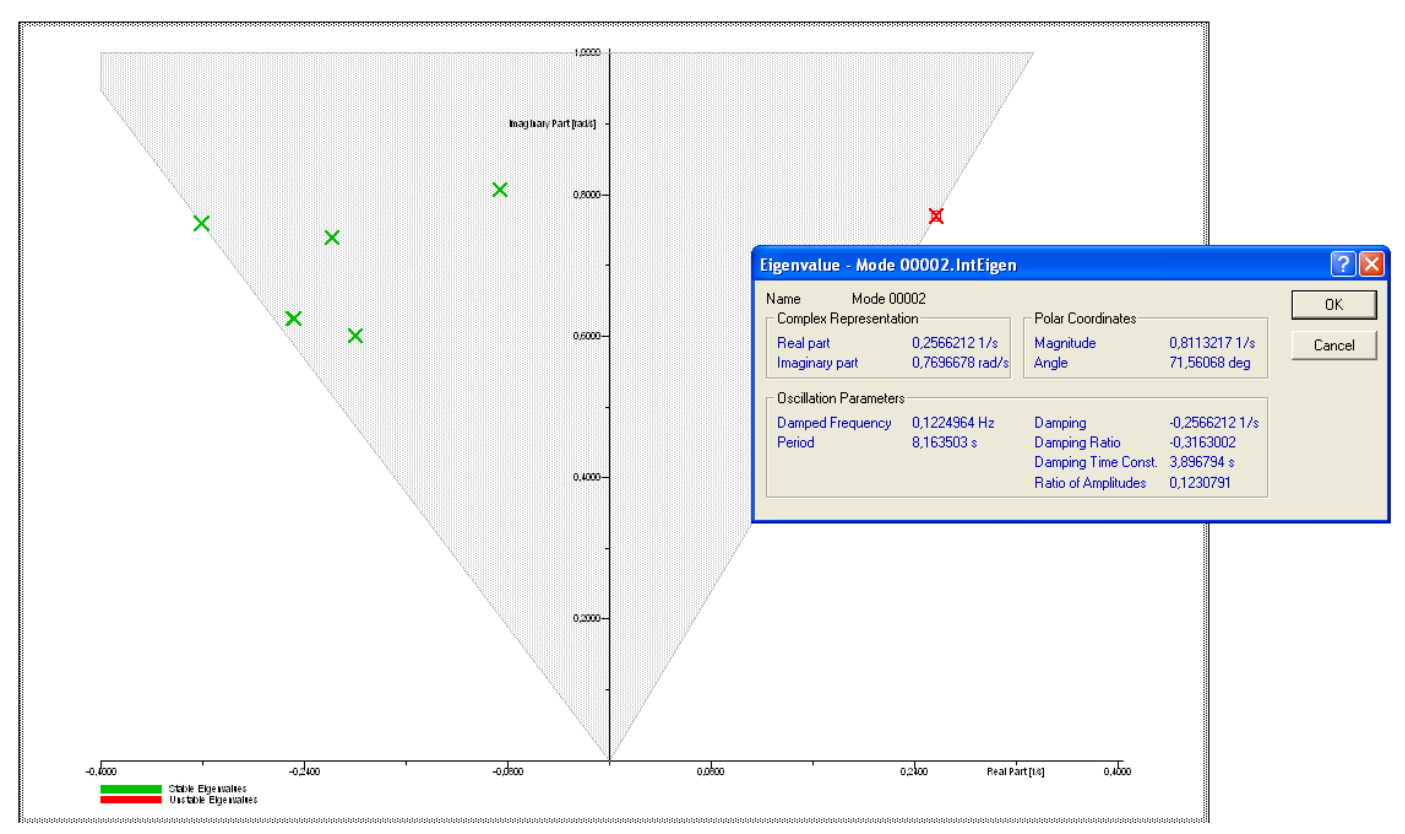

Fig. 2-30: Eigenvalues of the System Related to Rotor Speed 


\subsubsection{The Counter Measures against the Risk of Undamped Oscillations}

It is foreseen that the undamped oscillations as a result of negative damping introduced by the AVRs and unit governors may cause power swings which may cause separation of the two systems as shown by the simulation results in the previous part. Therefore, to preserve the rotor angle stability of the system, following precautions as illustrated in Fig. 2-31 have been recommended to TEIAS by UCTE working committee, in addition to retuning of the governors of hydroelectric power plants in order to remove the sources of negative damping, which is the focus of this study:

- Retuning of PSSs so as to damp $0.15 \mathrm{~Hz}$ inter area oscillations

- Modification of some STATCOMs to enhance damping

- Modification of some SVCs to enhance damping

- Installation of braking resistors as a back up to enhance damping

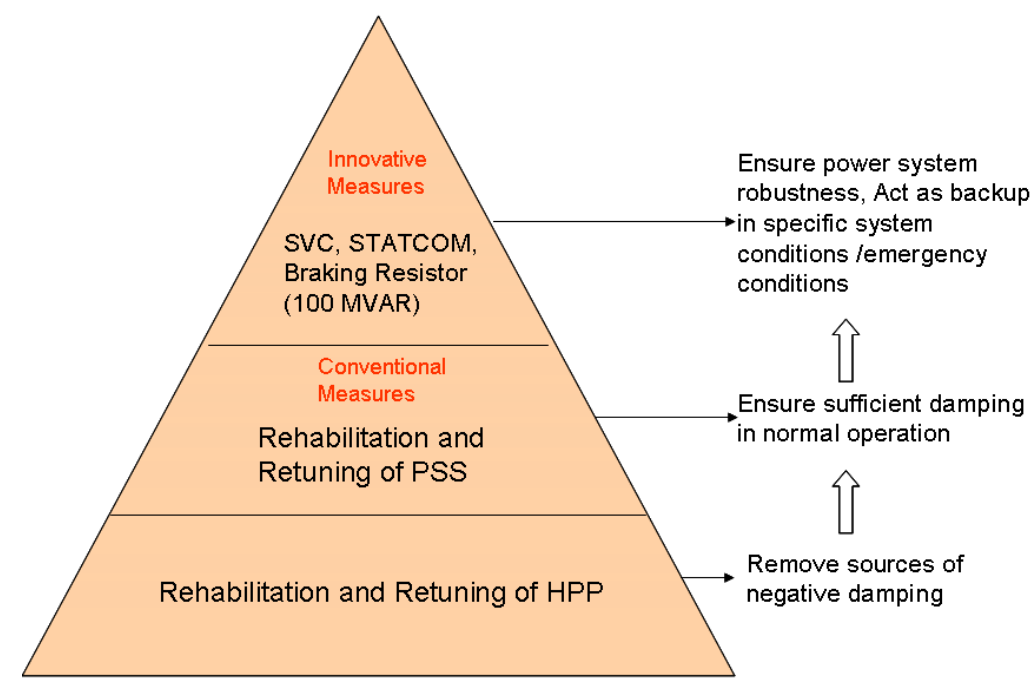

Fig. 2- 31: The Road Map to Preserve Rotor Angle Stability of the Interconnected System

The relative effect of the above mentioned damping measures was expected to be verified via simulation studies. 


\subsection{Contribution to the Problem Solution}

The main motivation of this study is to assess governor settings for hydroelectric power plants so as not to disturb -if possible- the damping of the machines and cause instability prior to interconnected operation. Afterwards, recommendations for operation of hydroelectric power plants prior to interconnected operation will be made.

In order to prepare a representative model for the Turkish Power System, a priority list of major power plants is formed [7]. Since the generation characteristic of the overall system is mainly determined by the major plants and it is infeasible to retune all installed hydroelectric power plants, it is assumed that retuning of governors of major hydroelectric power plants will ensure stable operation in addition to other counter measures listed in the previous part. The priority list of hydroelectric power plants is given in Table 2-2.

Table 2-2: The Priority List of Hydroelectric Power Plants

\begin{tabular}{|c|c|c|}
\hline Plant Name & Unit & Rating (MW) \\
\hline Atatürk & All & $8 \times 300$ \\
\hline Karakaya & All & $6 \times 300$ \\
\hline Birecik & All & $6 \times 126$ \\
\hline \multirow{2}{*}{ Keban } & $1-4$ & $4 \times 157$ \\
\cline { 2 - 3 } & $5-8$ & $4 \times 180$ \\
\hline Altınkaya & All & $4 \times 175$ \\
\hline Oymapınar & All & $4 \times 135$ \\
\hline Berke & All & $3 \times 175$ \\
\hline Hasan Uğurlu & All & $4 \times 125$ \\
\hline
\end{tabular}


In order to complete the retuning study, all hydroelectric power plants in the priority list are visited (except Keban which is in the scope of rehabilitation program hence does not contribute to frequency control) and site tests are performed, which are step response tests, frequency response tests and isolated operation tests. Since the formed models are verified via field test results, it has been accepted that the power plants are modeled accurately. Note that, some tests (frequency response tests and isolated operation tests) could not be performed on site in most of the power plants due to lack of test equipment. However, since the formed models are quite accurate, it has been accepted that simulation studies gave more than a rough idea about the frequency response tests and isolated operation tests.

For modeling hydroelectric power plants, a nonlinear version of IEEE HYGOV model in [12], is used and the control structure of governors are obtained from the manufacturers. Assumptions and methodology used in the modeling and validation process will be described in further chapters.

After obtaining a set of parameters of governor settings for a sample hydroelectric power plant via a single machine infinite bus model, the effect of the settings in multi machine systems is first verified via a modified four machine test system proposed by Kundur [14]. Afterwards, the list of governor settings of all priority list hydroelectric power plants is obtained and overall effect of the retuned governors of the priority list hydroelectric power plants is assessed using the ENTSO-E CESA System and Turkish Power System dynamical data provided by The University of Rostock, the Power Systems Department of TUBITAK UZAY and partially by TEIAS respectively, via time domain simulations and eigenvalue analysis.

After studying the effects of retuned hydro power plants' governor settings on the system damping, other counter measures that may have positive effects on damping of frequency oscillations are introduced as future studies, in addition, sample simulation studies are presented to have an idea about possible effects of these counter measures. 


\section{CHAPTER 3}

\section{MODELING OF THE HYDROELECTRIC UNITS AND THE TRANSMISSION NETWORK}

\subsection{Introduction}

The most crucial stage of engineering work in analysis of any system is the mathematical modeling process. The assumptions made throughout the mathematical modeling study determine the application range and reliability of the model. A very complicated system in practice may easily be modeled in a very simple and effective manner by making reasonable engineering assumptions. For example, a very common method for nonlinear system analysis is linearizing the system around an operating point, in case certain conditions are met. Although this method is successful for analyzing the effect of small perturbations around an operating point, it is far away from giving even a rough idea about the behavior of the system for large disturbances.

Almost any practical system is nonlinear in nature. Therefore, nonlinear system analysis knowledge and numerical simulation tools is essential in order to make reasonable engineering assumptions and effective mathematical modeling studies.

The main motivation of this study is to effectively model a hydroelectric unit so as to analyze and determine its behavior in case of dynamically varying system conditions. In order to achieve this goal, first the engineering assumptions regarding the modeling study of a hydroelectric power plant are justified. Afterwards, the mathematical model of the unit is formed according to the related dynamical data 
that has been surveyed and the model is verified by site measurements as an integral part of the study, using nonlinear simulation tools such as MATLAB Simulink. It is important to note here that every hydroelectric power plant is designed according to the specific geographical structure in the region of interest; hence no off the shelf universal nonlinear mathematical model satisfactorily representing the dynamical behavior of the plant is available. Therefore, the model validation study turns out to be a key part of the modeling process.

After a suitable set of parameters is achieved using a single machine infinite busbar model, the effectiveness of the proposed parameter set is assessed for multi machine case using Kundur's well known four machine, two area network [14]. Note that the two area multi machine model is simple enough to observe the effect of a control variable of a single machine in a multi machine power system without performing complicated sensitivity analysis and yet powerful enough to get a rough idea about the behavior of the plant by time domain simulations under dynamically varying (i.e., oscillatory) system conditions. By the results of this study, the locally optimized parameter set is concluded to be suitable.

Finally, the overall effect of the retuned hydroelectric power plants in the priority list is shown by eigenvalue and time domain analysis using the dynamical data given by the University of Rostock, which is comprised of the representative dynamical model data of ENTSO-E CESA network and complete dynamical data of Turkish Power System.

In this chapter of the thesis, related mathematical background and set of related differential equations will be formed in order to realize the above mentioned nonlinear modeling process and interpret the simulation results.

\subsection{Modeling Assumptions and Mathematical Preliminaries}

In order to successfully analyze a system, either experiments should be performed or detailed modeling process, which is essentially based on formation of differential 
equations regarding the system dynamics, should be carried out. In recent studies, with improved computer and software technology, the numerical simulation tools became an essential part of any scientific study.

In engineering, it is not possible to perform a study based solely on experiments due to time constraints and economical reasons. Especially in power systems engineering, it is reasonable to carry out a detailed modeling study as the first phase of each analysis or planning study.

As explained in Chapter 2, the power system dynamics are classified according to the time frame of interest. For example, in wave propagation studies, the time frame of interest is in the order of microseconds; therefore, it is useless to consider the effect of voltage controllers, the time frame of which is in the order of hundreds of milliseconds. This study is focused on assessment of the effect of governor parameters of hydroelectric power plants on sustained low frequency inter area oscillations. As stated in Chapter 2, after the synchronous interconnection of Turkish Power System with ENTSO-E CESA (former UCTE) System, it is expected that inter area oscillations within a frequency neighborhood of $0.15 \mathrm{~Hz}$ (dependent on system conditions) are prone to occur. Since the frequency of oscillations is too low as compared with the recorded ones in literature due to the large inertia of the overall system (generally such oscillations are recorded to be between the frequency range varying from $0.25 \mathrm{~Hz}$ to $0.8 \mathrm{~Hz}$ ), the frequency of the expected inter area oscillations turn out to be comparable with load frequency control band.

The expected mode of oscillation is in the control bandwidth of both the PSSs and the governors; therefore, in addition to modified PSS settings, it should be made sure that the contribution of speed governors together with the mechanical parts of the power plants to that phenomenon is prevented in order to improve, at least not to disturb the natural damping of the machines.

Apparently, the most important assumption made in this study is the time frame of interest. Since mechanical phenomena will be investigated, the time frame of interest 
is assumed to be in the order of seconds. This assumption will be justified by the measurements taken from the site tests in the preceding parts of the study.

A hydroelectric power plant is a nonlinear system which requires a wide variety of different disciplines of engineering from fluid mechanics to electromechanical energy conversion to model.

In governor tuning studies, the generator is not modeled since the electrical dynamics are so fast. It is assumed that the dynamics in the mechanical power of the turbine is observed from the electrical power that is measured from the generator terminals, after comparatively fast dynamics in load angle. In other words, comparatively fast electrical transients inherent in the generator are ignored in mechanical system modeling. However, in multi machine case simulations and in overall system studies including eigenvalue analysis, the electrical transients in the generators are also modeled together with the mechanical systems.

\subsubsection{Solution of Matrix Differential Equations}

Any power system is necessarily described by a high number of nonlinear differential equations. This part of the study will focus on the analysis of the oscillatory stability of such a large dynamic system using eigenvalue analysis. The main usage of eigenvalue analysis in power system studies is to simplify the analysis of a large dynamic system around an operating point by representing the system response to a disturbance as the linear combination of uncoupled aperiodic and oscillatory (periodic) responses, which are commonly referred to as the modes of the system.

A linear system, that has $\mathrm{n}$ inputs and $\mathrm{m}$ outputs, which is modeled by a set of differential equations, is well expressed with the set of matrix equations as in (3-1).

$$
\begin{aligned}
& \dot{x}=A x+B u \\
& y=C x+D u
\end{aligned}
$$


where,

$x$ denotes the (nx1) state vector comprised of the state variables of the system,

$A$ denotes the (nxn) matrix which is comprised of the coefficients of the state variables in differential equations modeling the system,

$B$ denotes the (nxn) matrix which is comprised of the coefficients of the input variables in differential equations modeling the system,

$u$ denotes the (nx1) vector comprised of the input variables of the system,

$y$ denotes the (mx1) output vector comprised of the output variables of the system,

$C$ denotes the (mxn) matrix which is comprised of the coefficients of the state variables in the equations expressing the output vector,

$D$ denotes the (mxn) matrix which is comprised of the coefficients of the input variables in the equations expressing the output vector.

The power system of interest is also going to be modeled with a set of nonlinear differential equations as will be discussed in preceding parts of this chapter. The system is going to be modeled around an operating point via linearization of the set of nonlinear differential equations that will have the same format as in (3-1). Therefore, the methods utilized in analyzing the general form of linear differential equations constitute a strong basis on system studies.

The set of differential equations is solved by first decoupling the A matrix by a transformation, then solving the decoupled system and finally expressing the state variables of interest by back transformation.

\subsubsection{Eigenvalues and Eigenvectors}

A number $\lambda$ is referred to as an eigenvalue of matrix $A$ if there is a nonzero column vector $x$ satisfying (3-2).

$$
A x=\lambda x
$$


The nonzero column vector in (3-2) is called the right eigenvector associated with eigenvalue $\lambda$. One can directly see that eigenvectors are not unique since the multiplication of any eigenvector with a constant also satisfies (3-2). Therefore, in practice eigenvectors are normalized so as to equal their magnitude to unity. The equation (3-2) can be ordered as in (3-3).

$$
(A-\lambda I) x=0
$$

The equation (3-3) has a nontrivial solution if and only if (3-4)is satisfied.

$$
\operatorname{det}(A-\lambda I)=0
$$

The equation (3-4) is called the characteristic equation of the system. The characteristic polynomial of an nth order system is of nth degree; therefore it has $n$ roots, $\lambda_{1}, \lambda_{2}, \ldots, \lambda_{\mathrm{n}}$, which are called the eigenvalues of the system.

The eigenvalues of a matrix may be real or complex or a combination of both.

Note that the equation (3-2) can also be as expressed as in (3-5). In this case, it is obvious that the eigenvalues will be the same. However, the eigenvectors will be different.

$$
w A=\lambda w
$$

The nonzero row vectors satisfying (3-5) are called the left eigenvectors of matrix $A$. Actually, if $P$ denotes the nxn matrix, the columns of which are formed by the right eigenvectors of the matrix $A$, namely $x_{1}, x_{2}, \ldots, x_{\mathrm{n}}$, then the nxn matrix $W=P^{-1}$ will be nothing but the matrix, the columns of which are formed by the left eigenvectors of the matrix $A$, namely $w_{1}, w_{2}, \ldots, w_{\mathrm{n}}$.

One important property of eigenvectors is that the right eigenvectors carry information about the observability of a mode (i.e., the observability of the modal 
variable on the state variable) whereas the left eigenvectors carry information about the controllability of a mode (i.e., the controllability of the modal variable using the state variable). It should also be noted that the left eigenvector of matrix $A$ is the right eigenvector of matrix $A^{\mathrm{T}}$. The participation factor of a state variable on an eigenvalue is the multiplication of its corresponding entry in the right eigenvector (i.e., the observability, commonly referred to as the mode shape) and the left eigenvector (i.e., the controllability). Participation factor is the sign of dependency of a mode on state variables and it is an important analysis tool on determination of problematic state variables (i.e., addressing the state variables causing instability) in large scale systems through sensitivity analysis.

\subsubsection{Solution of Matrix Differential Equations}

It is clear that from the equation (3-2), if the nxn matrix $P$, the columns of which is comprised of the right eigenvectors of matrix $A$, the following equation in (3-6) holds, where $\lambda$ denotes the diagonal matrix the elements of which are the eigenvalues of matrix $A$, respectively.

$$
A P=P \lambda
$$

It can easily be shown that, by directly multiplying both sides by $P^{-1}$, (3-7) is obtained.

$$
P^{-1} A P=\lambda
$$

Hence by defining the transformation $x=P z$, where $x$ denotes the state vector and $z$ denotes the modal vector, the coupled differential equation $\dot{z}=\lambda z$, can be solved in the specific decoupled vector space which is spanned by the eigenvectors of matrix $A$, and then the solution can be finalized by backward transformation. 


\subsubsection{Nonlinear Systems}

A nonlinear dynamic system is generally described by the differential matrix equation as in (3-8).

$$
\dot{x}=F(x, t)
$$

The equilibrium points $\hat{x}$ are those points where the system is at steady state, that is where all the state variables are constant, explicitly that $\mathrm{F}(\hat{x})=0$. Therefore, expanding $\mathrm{F}(\mathrm{x})$ in Taylor Series, the linear approximation of the nonlinear system can be made as in (3-9), in case, $\lim _{x \rightarrow 0}\left(\sup \frac{\left\|F(x, t)-\left.\frac{\partial F(x, t)}{\partial x}\right|_{\hat{x}} x\right\|}{\|x\|}\right)=0$ and the Jacobian $\left.\frac{\partial F(x, t)}{\partial x}\right|_{\hat{x}}$ is a bounded matrix.

$$
\Delta \dot{x}=\left.\frac{\partial F(x, t)}{\partial x}\right|_{\hat{x}} \Delta x
$$

By utilizing the indirect method of Lyapunov, if $\hat{0}$ is a uniformly asymptotically stable equilibrium point of (3-9) (i.e., if all eigenvalues of the matrix have negative real parts); then it is a uniformly asymptotically equilibrium point of (3-8).

It is important to note here that Lyapunov's Indirect Method does not arrive at any conclusions regarding the instability of the system. In order to assess the instability of the system, Lyapunov's instability theorem may be utilized. However, the applicability of the theorem is limited.

The above statement supports the weakness of eigenvalue analysis in concluding the instability of a nonlinear system as will be stated in the preceding parts of the study. Hence, the best way to analyze the nonlinear system case turns out to be the numerical simulation studies. 


\subsubsection{Periodic Solutions of Nonlinear Systems with Informal Arguments}

In nonlinear system analysis, it is a very common practice to express a system using the system configuration with a dominant nonlinearity as illustrated in Fig. 3-1.

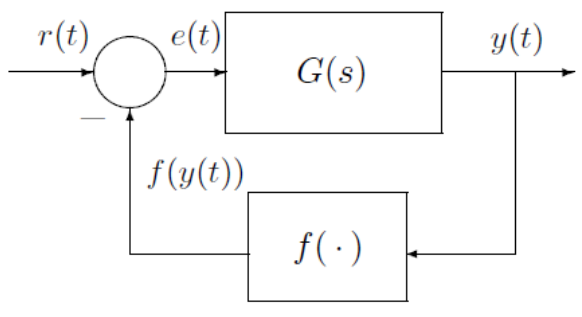

Fig. 3-1: Nonlinear System Configuration with a Dominant Nonlinearity

In Fig. 3-1, the system denoted by $\mathrm{G}$ is a Linear Time Invariant (LTI) system, whereas $\mathrm{f}$ is a nonlinear system with the assumptions that,

(i) $\mathrm{G}$ is Bounded Input Bounded Output Stable (BIBO Stable),

(ii) Gr has finite average power as long as $r$ has finite average power,

(iii) The nonlinear system $f$ maps $F$ into itself,

(iv) The nonlinear system $f$ has a describing function $\eta(a, w)$, where a corresponds to the magnitude and $\mathrm{w}$ corresponds to the frequency of the input.

Note that a describing function is an optimal quasi linearization (i.e., the best linear approximation) of the nonlinear system with respect to a sinusoidal input, such that the steady state component of the representative linear system is equal to the first harmonic of the nonlinear system output.

In physical systems, $\mathrm{G}$ behaves like a low pass filter which eliminates higher order harmonics created by the nonlinearity; hence the output of the linear system is essentially close to a pure sinusoid. 
In phasor domain, the above explained phenomenon is expressed as in (3-10).

$$
1+\hat{g}\left(j w_{0}\right) \eta\left(a, w_{0}\right)=0
$$

The equation (3-10) is commonly referred to as the principle of harmonic balance.

Therefore, it can be concluded that a given nonlinear system can be approximated by a Linear Time Invariant System. The optimal way of representing nonlinearity is utilizing its describing function, which is the optimal quasi linearization of the nonlinearity (i.e., the best approximating linear system to the nonlinear system). Moreover, due to the low pass nature of practical physical systems, the first harmonic of the response of the nonlinear system can be considered as the response of the overall system to the sinusoidal input.

\subsection{Modeling Hydroelectric Units}

Regardless of the type of engineering work to be performed, (i.e., whether practical site work or planning studies), a detailed analysis study should be performed in advance. As stated before, the most crucial stage of engineering work in analysis study of any system is the mathematical modeling process. The assumptions made throughout the mathematical modeling study determine the application range and reliability of the model or experiment. A very complicated system in practice may easily be modeled in a very simple and effective manner by making reasonable engineering assumptions therefore significantly increasing the efficiency of the further work. Moreover, as stated before, in engineering, it is not possible to perform a study based solely on experiments due to time constraints and economical reasons. Especially in power systems engineering, with developing computer and software technology, it has become reasonable to carry out a detailed modeling study as the first phase of each analysis or planning study.

The main motivation of this part of the study is to achieve a set of differential equations in order to develop an effective dynamic mathematical model of a hydroelectric power plant so as to analyze and determine its behavior in case of 
varying system conditions or plant specific disturbances. It is important to note here that every hydroelectric power plant is designed according to the specific geographical structure in the region of interest; hence no off the shelf universal nonlinear mathematical model satisfactorily representing the dynamical behavior of the plant is available, which introduce the essence of site tests in model validation process.

\subsubsection{The Structure of a Hydroelectric Power Plant}

Basically, a hydroelectric power plant utilizes the energy of a river to generate electricity via driving the water turbine. Therefore, either the potential energy of the river or the kinetic energy of the river should be utilized to generate electricity which constitutes the basic principle of all kinds of hydroelectric power plants.

A hydroelectric power plant may be dam type where the water is stored at a reservoir behind a huge dam and the electricity is produced using the potential energy of the dammed water. In this case, the energy extracted from the water depends on the flow and on the difference in height between the head water level and the tail water level of the water. This height difference is called the hydraulic "head" available. The amount of potential energy in water is proportional to the head. To deliver water to a turbine while maintaining pressure arising from the head, a large pipe called a penstock may be used as illustrated in Fig. 3-2 [30].

A hydroelectric power plant may also have no reservoir capacity which are called run of the river type, therefore it is not possible to store water in such kind of hydroelectric power plants.

Pumped storage type hydroelectric power plants generate electricity to supply high peak demands by moving water between reservoirs at different elevations. At times of low electrical demand, excess generation capacity is used to pump water into the higher reservoir. When there is higher demand, water is released back into the lower reservoir through a turbine [30]. 


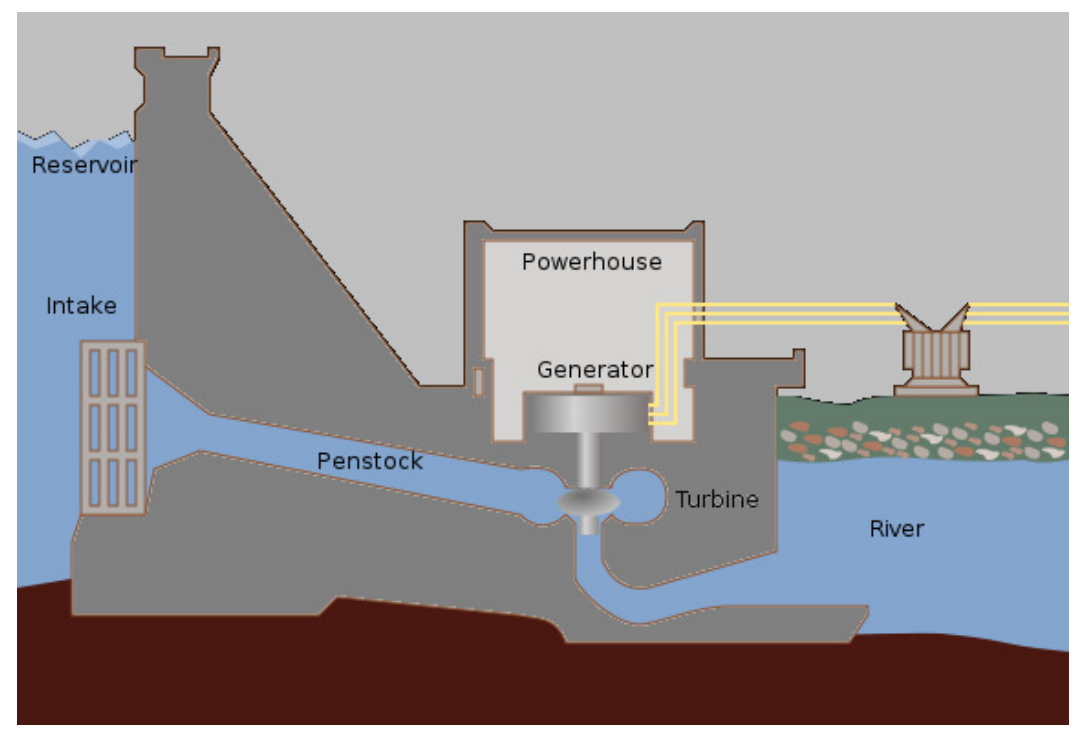

Fig. 3-2: The Structure of a Dam Type Hydroelectric Power Plant [30]

A tidal hydroelectric power plant makes use of the daily rise and fall of the water due to tides. Such plants can also be dispatchable if it is possible to construct reservoirs to the specific site of interest.

Channel type hydroelectric power plants use the kinetic energy of a river to generate electricity. Such plants have a very limited reservoir capacity. Therefore, channel type hydroelectric power plants are commonly referred to as a regulator.

\subsubsection{Electromechanical System Modeling}

\subsubsection{Turbine and Penstock Modeling}

Water turbines constitute one of the oldest means of mechanical energy conversion devices. The basic principle of hydraulic turbines is to change the tangential momentum of the water passing through the blades of a runner so as to create a tangential force on the runner; hence transferring the energy of water through the shaft to the generator which realizes the electromechanical energy conversion. The water is then discharged with reduced energy. 
The two basic types of water turbines are the impulse and the reaction type of hydraulic turbines. The reaction turbines are acted on by water, which changes pressure as it moves through the turbine and gives up its energy; whereas, the impulse turbines change the velocity of a water jet by a nozzle. The pressurized water impinges on the turbine's curved blades changing the direction of the flow, therefore creating a change in momentum (impulse), hence a force on the turbine blades causing mechanical energy conversion. The reaction turbines are utilized in case of medium $(30 \mathrm{~m}<\mathrm{h}<300 \mathrm{~m})$ or low $(\mathrm{h}<30 \mathrm{~m})$ head; whereas the impulse turbines are utilized in case of high head $(\mathrm{h}>300 \mathrm{~m})$ conditions. The turbine application chart containing the application range of various types of turbines is illustrated in Fig. 3-3 [30].

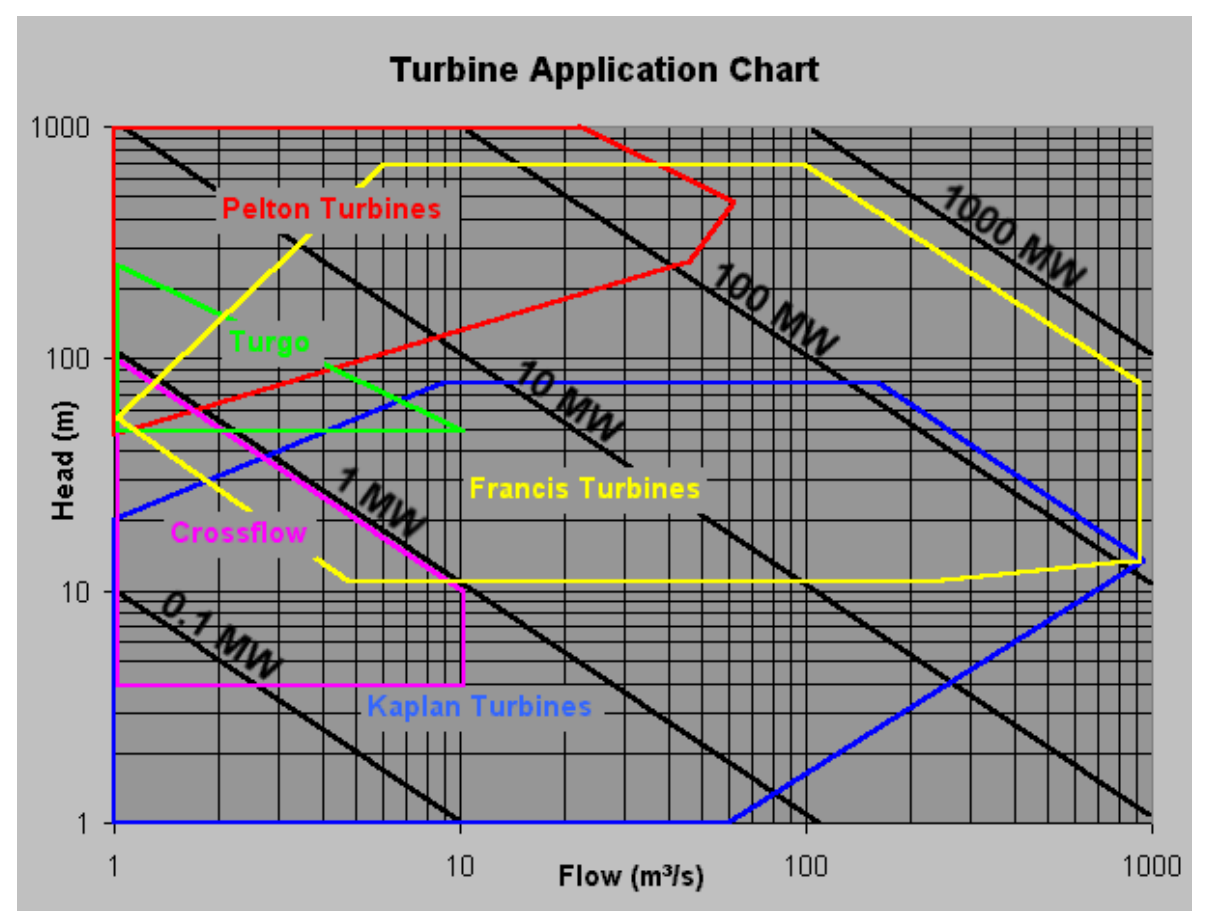

Fig. 3-3: Turbine Application Chart [30]

As stated in the previous part, there are many practical types of hydroelectric power plant applications. However, since the scope of this study is the retuning of the hydroelectric power plants in the priority list so as not to deteriorate system damping, 
only the detailed modeling of Francis Type of turbines will be investigated since all hydroelectric power plants in the priority list are equipped with Francis Turbines.

The general structure of a vertical generating unit equipped with a Francis Turbine is illustrated in Fig. 3-4 and the elements of a Francis turbine are illustrated in Fig. 3-5.

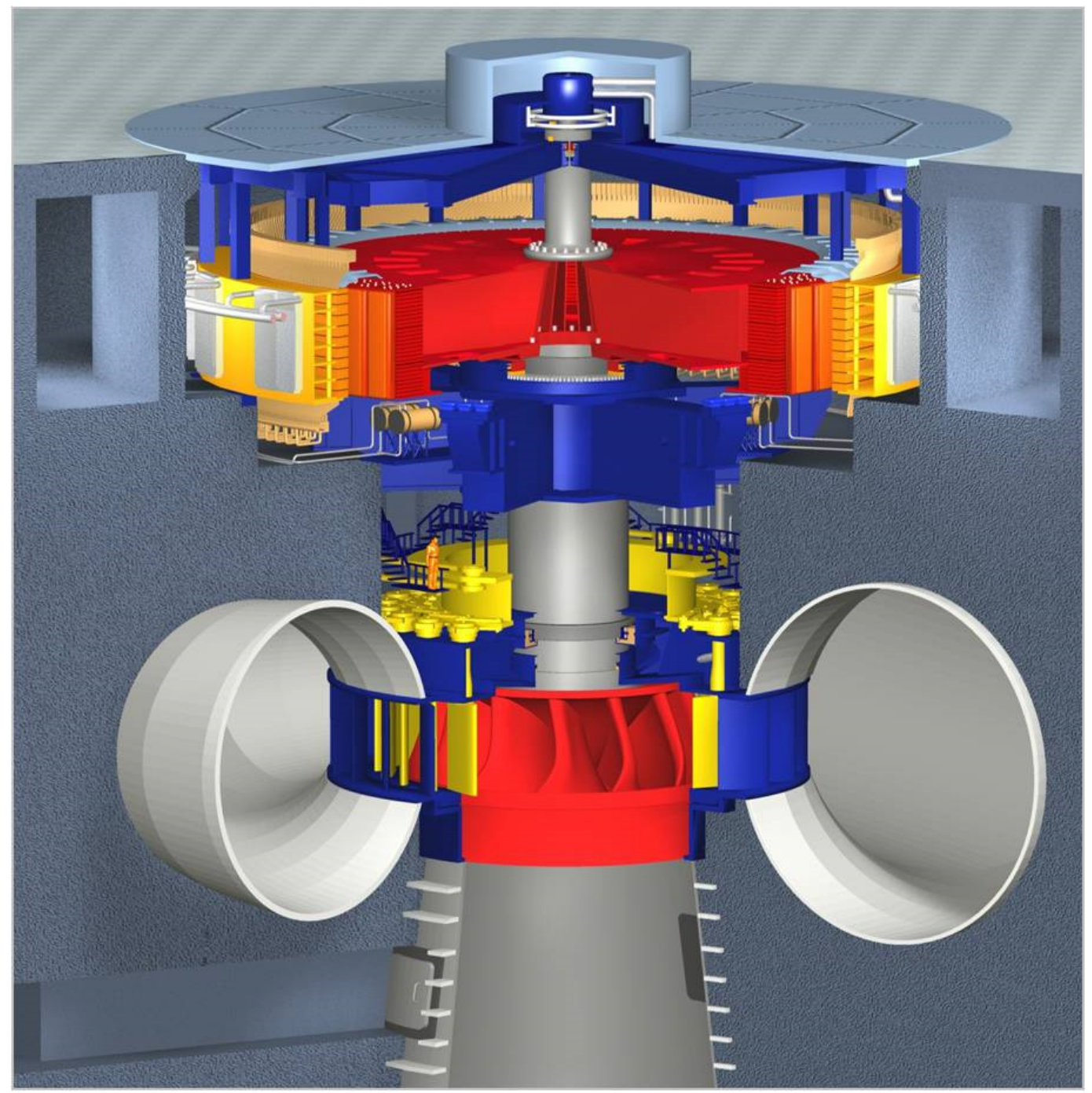

Fig. 3-4: The Structure of a Vertical Generating Unit Equipped with a Francis Turbine [37] 


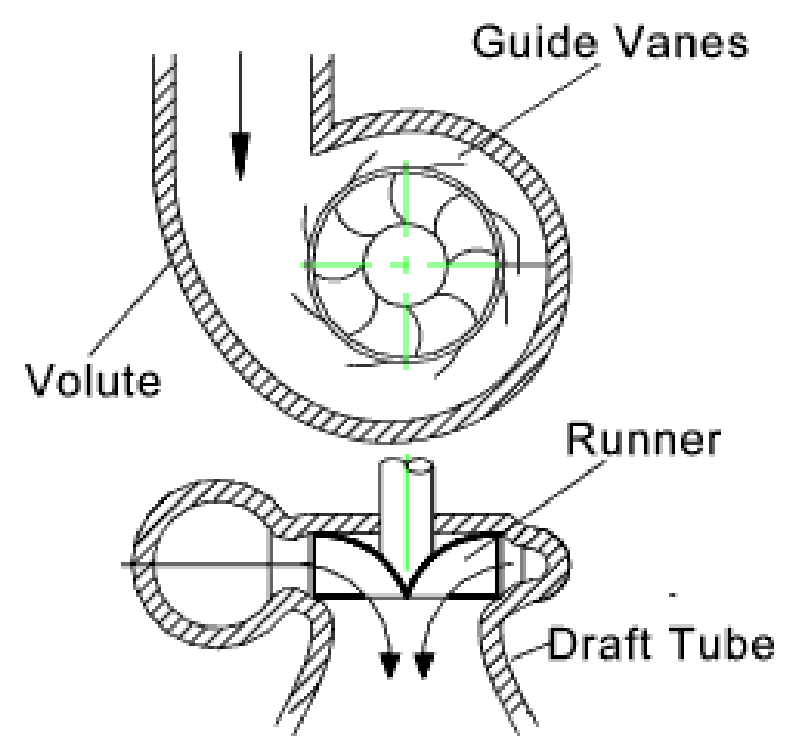

Fig. 3-5: The Most Basic Elements of a Francis Turbine

The turbine and penstock characteristics are determined by four basic relations between the turbine mechanical power, velocity of water in the penstock and turbine inlet (in per unit notation velocity also corresponds to flow rate) and the acceleration of the water column.

The mechanical power that can be transferred to the generator shaft from the Francis Turbine is a nonlinear function related to the flow rate (q) and hydraulic pressure which is strongly dependent on hydraulic head available (h). The nonlinear relationship is expressed via an efficiency term as a function of head and flow rate and the mechanical power of a turbine is expressed as in (3-11).

$$
P_{m}(q, h)=\eta(q, h) q \rho g_{a} h
$$

where,

$\mathrm{P}_{\mathrm{m}}$ denotes the mechanical power of the turbine (W)

$\eta$ denotes the efficiency factor

q denotes the flow rate $\left(\mathrm{m}^{3} / \mathrm{sec}\right)$

$\rho$ denotes the density of water $\left(\mathrm{g} / \mathrm{m}^{3}\right)$ 
$\mathrm{g}_{\mathrm{a}}$ denotes the gravitational acceleration constant $\left(\mathrm{m} / \mathrm{s}^{2}\right)$

$\mathrm{h}$ denotes the hydraulic head $(\mathrm{m})$.

The output power of the Francis turbine is adjusted by changing the opening of wicket gates, hence the amount of water flowing into the runner blades. As the opening of the wicket gate changes, the effective flow area of the water changes; therefore, the inlet water velocity in the penstock, hence the inlet water flow to the turbine runner changes. This relationship in per unit is as expressed in (3-12).

$$
q=A \sqrt{h}
$$

where,

q denotes the water flow (per unit)

A denotes the effective flow area (per unit)

$\mathrm{h}$ denotes the hydraulic head available (per unit)

There are two dominant nonlinearities in the hydraulic turbine model proposed in [8], which is utilized throughout this study. The remaining equations regarding the hydraulic turbine model are nothing but a restatement of the well known HYGOV model [12], [13].

The first nonlinearity in the model is the relationship between the actual gate opening and effective flow area. The no load losses of the turbine is included in the modeling process via subtracting the gate opening corresponding to no load flow from the actual gate opening and also the nonlinear relationship between gate opening and effective flow area due to geometry of the turbine is included in the nonlinear function expressed in (3-13).

$$
A=f(G)
$$

where,

A denotes the effective flow area (per unit) 
$\mathrm{f}$ denotes the nonlinear function relating gate opening and effective flow area $\mathrm{G}$ denotes the wicket gate opening (per unit)

A sample graph representing the nonlinear relationship between gate opening and effective flow area is illustrated in Fig. 3-6.

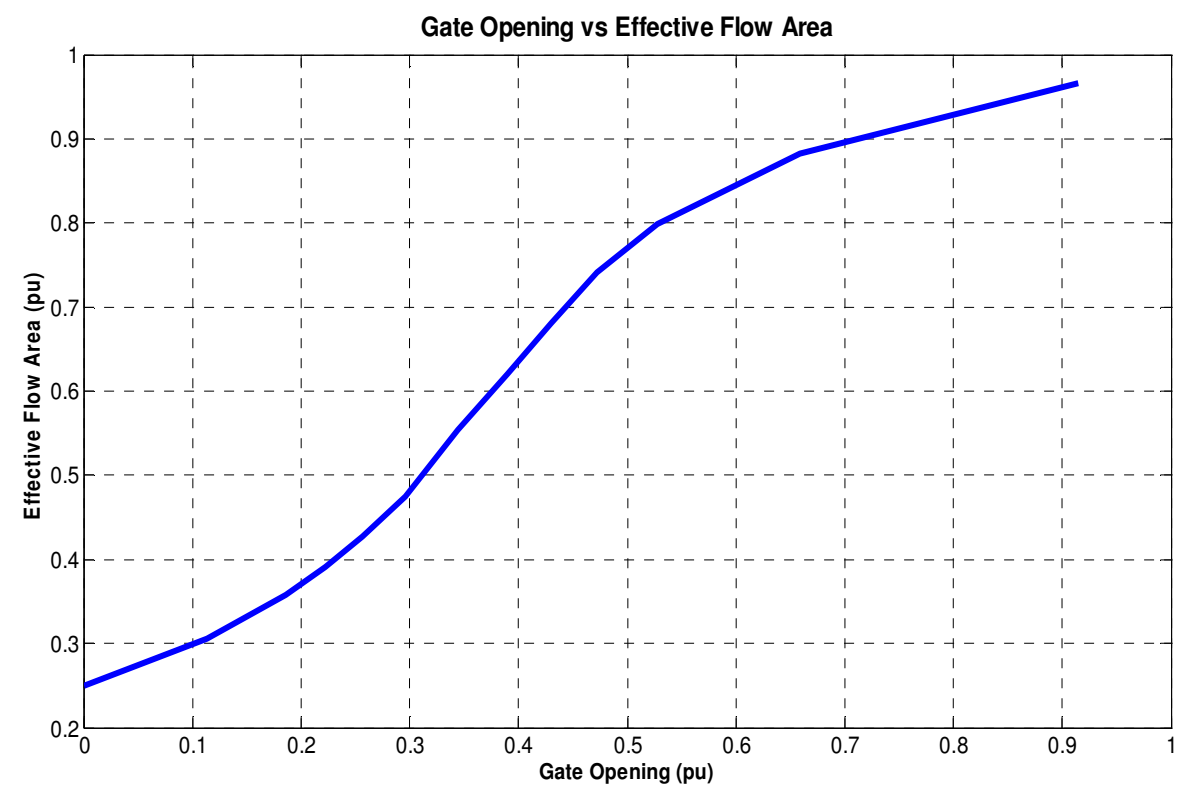

Fig. 3-6: A Sample Gate Opening and Effective Flow Area Relationship

The second nonlinearity in the turbine model is the nonlinear relationship between the mechanical power, the water flow and the hydraulic head. This nonlinearity is expressed by using the "Shell Curve" (a.k.a. Hill Chart, Performance Chart or Efficiency Curve), which expresses the output mechanical power of the turbine as a function of the head and the water flow as illustrated in Fig. 3-7.

Shell curves are obtained in the model prototype tests of hydraulic turbines in specific laboratories. In the shell curve, the solid lines correspond to the loci of operating points with equivalent efficiency, whereas the dashed lines correspond to the loci of operating points corresponding to equivalent mechanical power. The operational limits of the hydraulic turbine are also illustrated in the shell curves. 
In this study, the shell curve will be utilized to represent the nonlinear relation between output power, hydraulic head and water flow rate. Specific points will be selected from different regions of the shell curve and the relation for the remaining operating points will be found by piecewise linear interpolation between the selected points. Hence the accuracy of the nonlinear surface in representing the nonlinear relation between output power, hydraulic head and water flow rate is strongly dependent on the number and spectrum of the selected operation points from the shell curve.

The nonlinear relationship relating mechanical power output with water flow and hydraulic head is as expressed in (3-14).

$$
P_{m}=g(q, h)
$$

where,

$\mathrm{P}_{\mathrm{m}}$ denotes the mechanical power of the turbine (per unit)

$\mathrm{g}$ denotes the nonlinear function relating mechanical power to water flow and hydraulic head, as expressed in (3-11)

$\mathrm{h}$ denotes the hydraulic head (per unit)

A sample graph representing the nonlinear relationship relating mechanical power to water flow and hydraulic head obtained from the shell curve is illustrated in Fig. 3-8. 


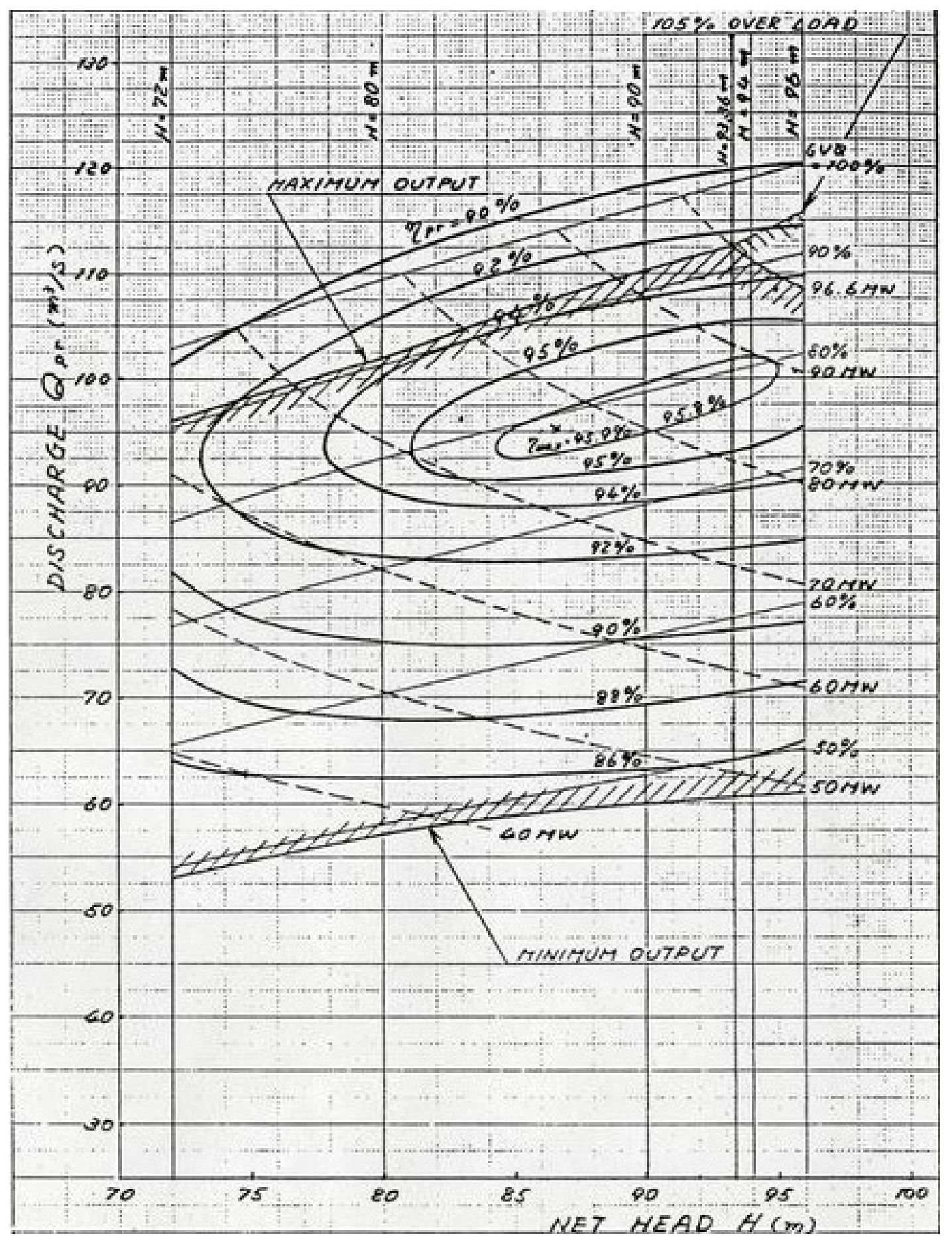

Fig. 3-7: A Sample Shell Curve 


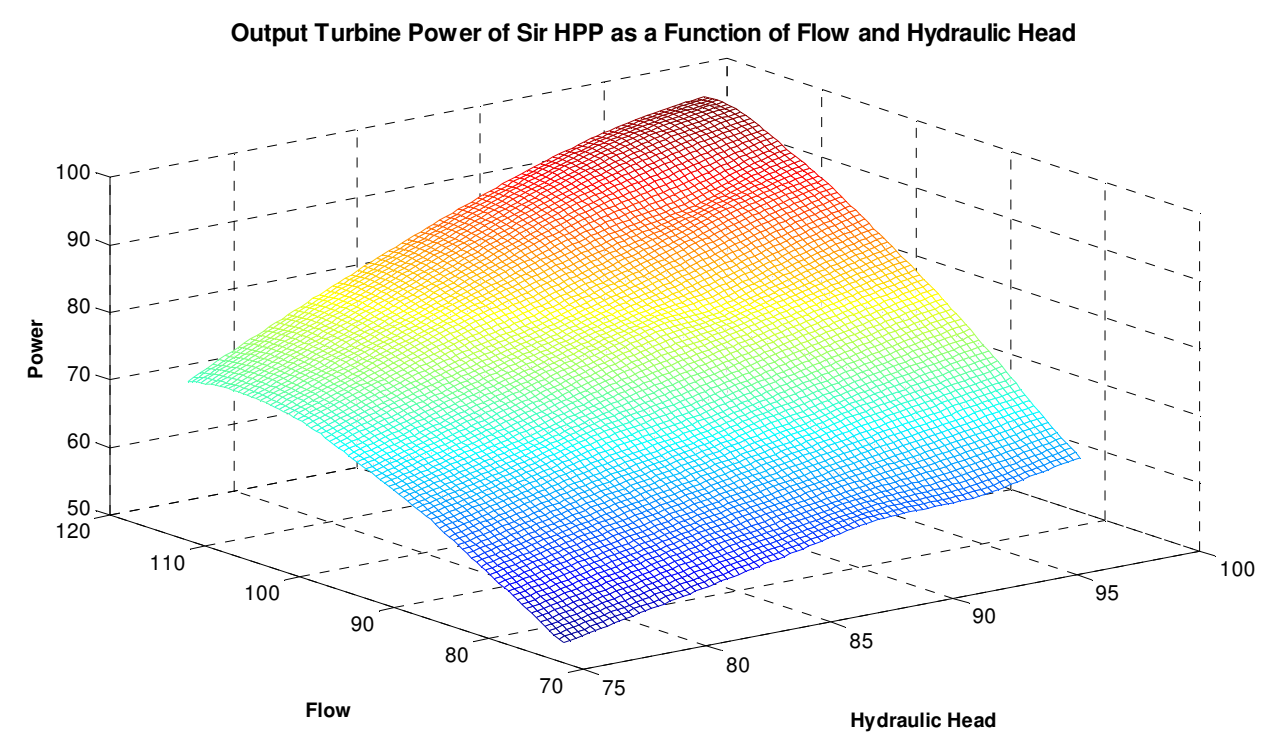

Fig. 3-8: A Sample Graph of Mechanical Power as a Function of Flow and Hydraulic Head

The third equation regarding the nonlinear turbine model is related to the acceleration of the water column. The characteristics of the water column in the penstock feeding the turbine carry great importance in representing the dynamical behavior of the hydraulic turbine.

The most important parameter in representing the characteristics of the water column is the inertia of water in the penstock. This inertia causes a great amount of lag for changes in mechanical power against changes in wicket gate opening. In fact, the power has a transient response which is initially in the opposite sense to that intended by changing the guide vane position. Although the turbine guide vane opening may change rapidly, the water column inertia prevents the flow from changing as rapidly. Consequently, after a rapid increase in guide vane opening, and before the flow has had time to change appreciably, the velocity of water into the wheel drops because of the increased area of the guide vane opening. The power transfer to the wheel actually drops before it increases to its required steady state value. This is the most prominent factor, which makes a hydraulic turbine such an uncooperative component in a speed control system [9]. 
The most basic water column model as expressed in [2] represents a single penstock with a very large or no surge tank so that no changes in reservoir head occur in response to flow changes. A hydraulic turbine model developed for a hydroelectric power plant containing a surge tank can be investigated from [23]. While developing the penstock model, it is assumed that the water acts as incompressible fluid so that here the water hammer effect may be neglected.

The penstock head losses due to the friction of water against the penstock wall are proportional to flow squared as expressed in (3-15).

$$
h_{f}=r q^{2}
$$

where,

$\mathrm{h}_{\mathrm{f}}$ denotes the head loss due to friction (per unit)

$\mathrm{r}$ denotes the friction coefficient

q denotes the flow through the penstock (per unit)

Considering the water column in the penstock as a solid mass, the rate of change of flow in the penstock is related to the pressure, hence the hydraulic head available, using Newton's $2^{\text {nd }}$ law of motion as in (3-16).

$$
\left(h_{0}-h-h_{f}\right) \rho g_{a} A_{p}=l A_{p} \rho \frac{d v}{d t}
$$

where,

$\mathrm{h}_{0}$ denotes the gross head $(\mathrm{m})$

$\mathrm{h}$ denotes the head at the turbine admission (m)

$\mathrm{h}_{\mathrm{f}}$ denotes the head loss due to friction $(\mathrm{m})$

$\rho$ denotes the density of water $\left(\mathrm{g} / \mathrm{m}^{3}\right)$

$\mathrm{g}_{\mathrm{a}}$ denotes the gravitational acceleration constant $\left(\mathrm{m} / \mathrm{s}^{2}\right)$

$A_{p}$ denotes the cross sectional area of the penstock $\left(\mathrm{m}^{2}\right)$

1 denotes the length of the penstock $(\mathrm{m})$

$\mathrm{v}$ denotes the speed of the water column in the penstock $(\mathrm{m} / \mathrm{s})$ 
Since the area of the penstock is constant and it is assumed that the flow of water is laminant (i.e., turbulance does not occur), the multiplication of the speed of the water column with the area of the penstock gives the rate of flow of water in the penstock. Hence, (3-16) can also be expressed as in (3-17).

$$
\frac{d q}{d t}=\left(h_{0}-h-h_{f}\right) \frac{g_{a} A_{p}}{l}
$$

In per unit notation, (3-17) is expressed as in (3-18) and in (3-19).

$$
\begin{aligned}
& \frac{d \bar{q}}{d t}=\left(1-\bar{h}-\bar{h}_{f}\right) \frac{h_{b a s e} g_{a} A_{p}}{l q_{b a s e}} \\
& \frac{d \bar{q}}{d t}=\frac{\left(1-\bar{h}-\bar{h}_{f}\right)}{T_{w}}
\end{aligned}
$$

where, $T_{w}=\frac{l q_{\text {base }}}{g_{a} A_{p} h_{\text {base }}}=\frac{l v_{\text {base }}}{g_{a} h_{\text {base }}}$ is the water starting time at rated load. It is important to note here that the water starting time varies with load. The water starting time represents the time required for a head $h_{\text {base }}$ to accelerate the water in the penstock from standstill to the velocity $\mathrm{v}_{\text {base. }}$ This is calculated between turbine inlet and the forebay or the surge tank if a large one exists [12].

The equation (3-19) constitutes an important characteristic of the mechanical power response of a hydroelectric power plant against wicket gate opening changes. If the wicket gate is closed for a certain amount, a back pressure will arise causing the water to decelerate. That is, if there is a positive pressure change, there will be a negative acceleration change. Similarly, a negative pressure change will cause a positive acceleration change.

Water starting time at any loading is related to the water starting time at rated load by the equation (3-20). 


$$
T_{w}=\frac{q_{0} h_{r}}{q_{r} h_{0}} T_{w_{\text {rated }}}
$$

While developing the penstock model, it was assumed that the water acted as an incompressible fluid so that the water hammer effect could be neglected. However, for some power system studies, it may be necessary to express the dynamic relation between the hydraulic system and the power system. Considering the water compressibility and pipe elasticity, the water dynamics can be written in per unit notation as in (3-21) and in (3-22) for a hydroelectric power plant with a large reservoir or a very large surge tank as illustrated in Fig. 3-9. It is important to note here that the term "s" in (3-21) and in (3-22) correspond to the Laplacian operator. The equations (3-21) and (3-22) are utilized to analyze the effect of travelling waves with typical frequency range around $1 \mathrm{~Hz}$ in the penstock and hence very similar to the long transmission line equations used in analyzing wave phenomena in power systems.

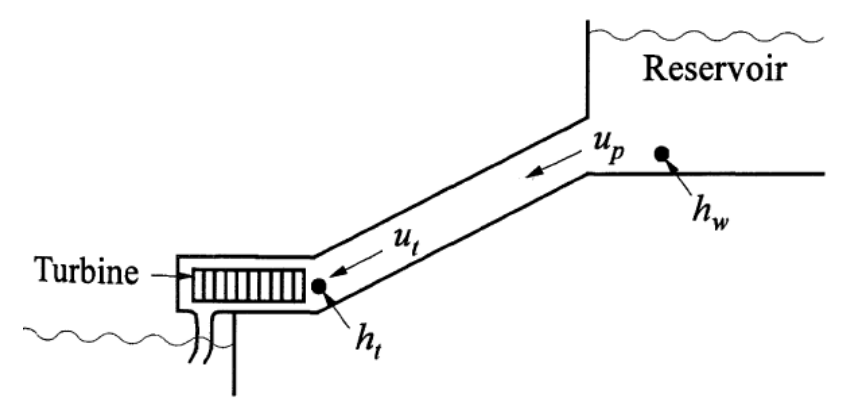

Fig. 3-9: The Schematic of a Hydroelectric Power Plant with a Reservoir

$$
\begin{aligned}
& \bar{h}_{t}=\bar{h}_{w} \operatorname{sech}\left(T_{e} s\right)-Z_{n} \bar{q}_{t} \tanh \left(T_{e} s\right)-r \bar{q}_{t}^{2} \\
& \bar{q}_{p}=\bar{q}_{t} \cosh \left(T_{e} s\right)+\frac{1}{Z_{n}} \bar{h}_{t} \sinh \left(T_{e} s\right)
\end{aligned}
$$

where, 
$\mathrm{h}_{\mathrm{t}}$ corresponds to the head at turbine admission (per unit)

$\mathrm{h}_{\mathrm{w}}$ corresponds to the gross head (per unit)

$\mathrm{T}_{\mathrm{e}}$ corresponds to the elastic time of water (s)

$\mathrm{Z}_{\mathrm{n}}$ corresponds to the hydraulic impedance of the penstock (per unit)

$\mathrm{q}_{\mathrm{t}}$ corresponds to the flow rate in the turbine inlet (per unit)

$\mathrm{q}_{\mathrm{p}}$ corresponds to the flow rate in the penstock (per unit).

Also it should be noted that, $T_{e}=\frac{l}{\text { wave velocity }}$ and $Z_{n}=\frac{q_{\text {rated }}}{h_{\text {rated }}} \frac{\text { wave velocity }}{A_{p} g_{a}}$, where typical values for wave velocity of water is $1220 \mathrm{~m} / \mathrm{s}$ for steel penstocks and 1420 $\mathrm{m} / \mathrm{s}$ for rock penstocks and 1 is the length of the penstock.

Taking the first two terms of the Taylor series expansion of the equations (3-21) and (3-22) ends up with the final equation regarding the penstock dynamics expressed in frequency domain as (3-23).

$$
h_{t}=\frac{2 T_{w}}{T_{e}^{2} s}\left(q_{p}-q_{t}\right)
$$

Hence, the combined model of turbine and penstock is illustrated in Fig. 3-10.

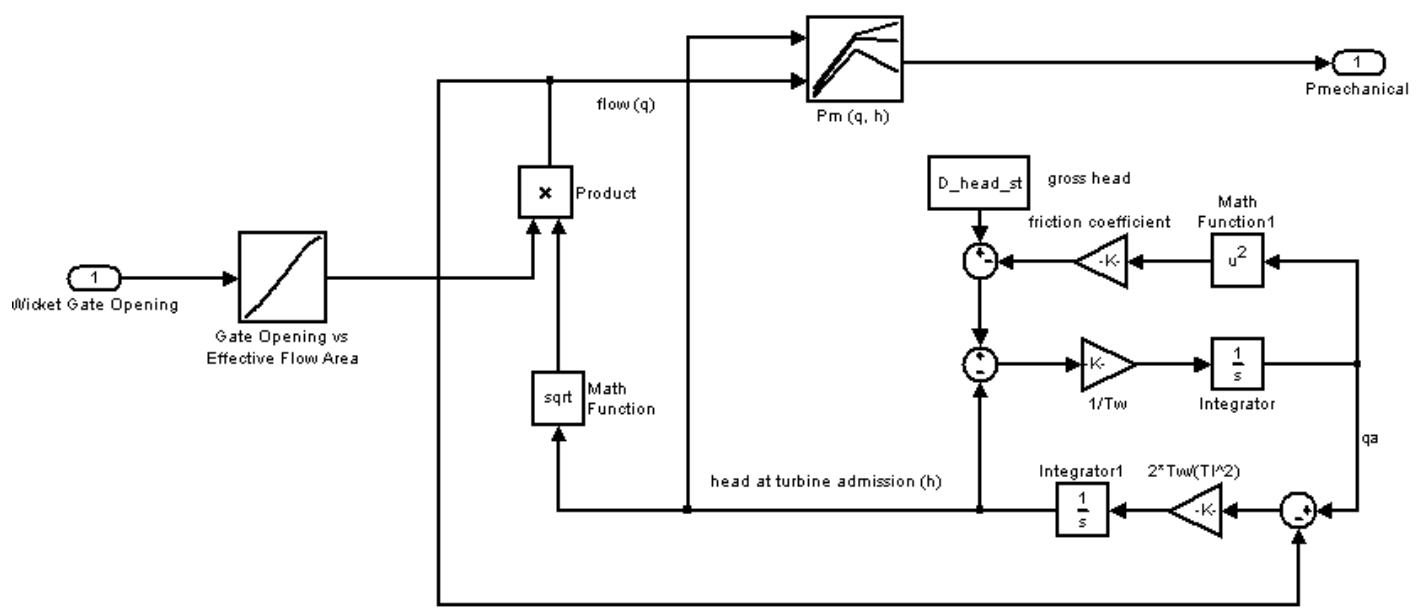

Fig. 3-10: The Combined Turbine and Penstock Dynamical Model 


\subsubsection{Governor Modeling}

The basic function of a governor is to control load (and/or speed for some specific cases) of a synchronous machine according to the principles of Load Frequency Control (LFC) explained in 2.3.1.

The primary aim of the governor concerning the LFC operation is the realization of primary frequency control. Primary frequency control involves responding of generating units against grid frequency deviations, hence controlling gate (or vane) positions by feeding back speed error. Therefore, in modern power systems, in order to maintain satisfactory and stable parallel operation of multiple units, the droop characteristic, which is explained in Section 2.3.1.1. in detail, is necessary to ensure equivalent load sharing between generating units. Moreover, such a primary frequency control philosophy is inevitable in bulk power systems, where a single unit (or plant) is insufficient to regulate the frequency of the whole system in addition to the high probability of facing with transmission bottleneck situations after some outage scenarios those will eventually end up with cascaded outages.

Therefore, a proportional output power response against frequency deviations, determined by the droop characteristics of the individual unit is expected from the individual generating units. However, such a control structure is inappropriate for a hydroelectric power plant due to the peculiar transient response of the unit against fast gate opening changes. As shown in (3-19), if there is a positive pressure change, there will be an initially negative acceleration change. Similarly, a negative pressure change will cause an initial positive acceleration change.

This false initial action may cause instabilities in case it is not compensated as explained in [5]. The compensation is provided by rate feedback, commonly referred to as transient gain reduction, thus retarding fast gate movements proportional to frequency deviations as a result of droop characteristics. Hence, for stable control performance, a large transient droop with long resetting time is essential. Therefore, the basic control strategy for a hydroelectric unit is high droop (low gain) operation 
for fast speed deviations and low droop (high gain) in steady state operation as explained in detail in [5]. This phenomenon is as shown in Fig. 3-11 for a typical hydroelectric unit equipped with a governor with permanent droop and transient gain reduction characteristics.

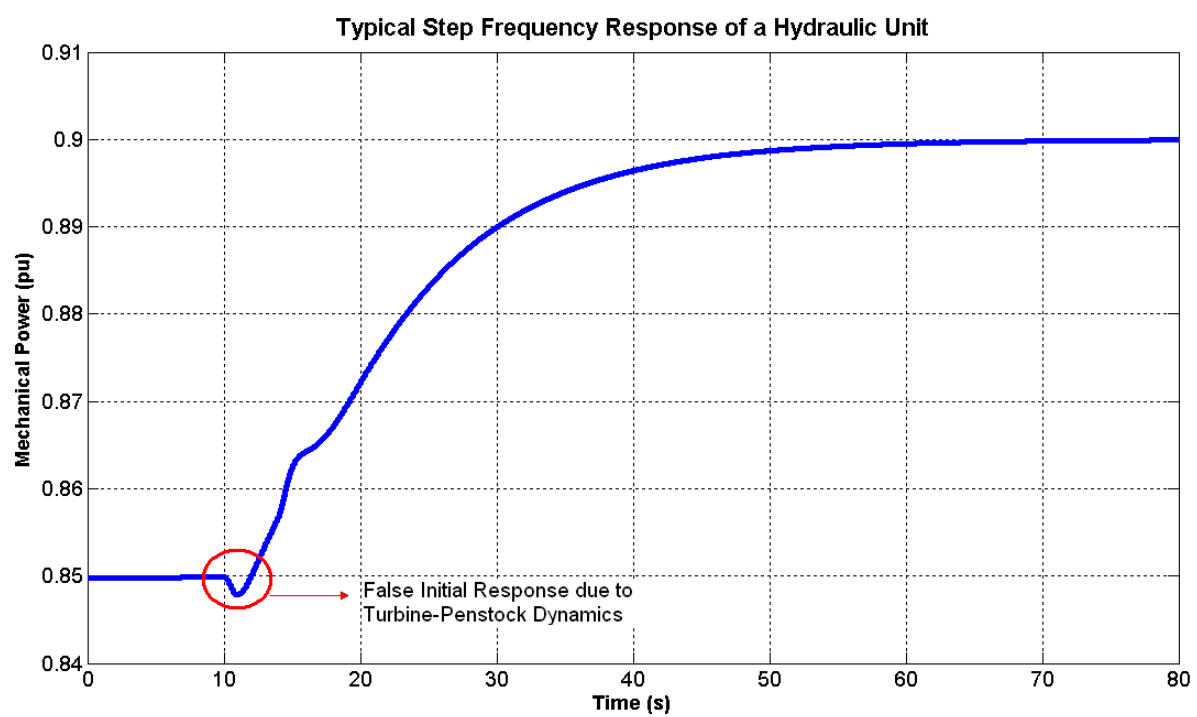

Fig. 3-11: Typical Step Frequency Response of a Hydroelectric Unit

The above explanations clearly show that the hydraulic turbines have some special requirements in terms of both the theoretical and the physical realization of governors. In addition to the controller of the governor, there is also the electrohydraulic system, that maintains the pneumatic operation of the gates and servomotors which realize the actual response of the mechanical system, hence causing changes in wicket gate opening with respect to the movement of pilot valves controlled by actuators. For the scope of this study, it is sufficient to model the electrohydraulic system with first order delays and integrators with appropriate time constants and limiters.

There are mainly two mainstream control algorithms for hydraulic governors in literature. Although there are many applications in practice, the basic philosophy of 
control is the same as the ones in literature. The most common speed governor is commonly referred to as a PD governor which maintains proportional control with transient droop as illustrated in Fig. 3-12. The other type of hydraulic speed governors is called a PID governor as illustrated Fig. 3-13. The speed governors of all hydroelectric power plants in the previously mentioned priority list [7] are variations of PD type hydraulic governors. Hence this type of hydraulic governor will be of concern throughout this study.

In the modern speed governors, there are several modes of operation regarding different operational concerns. However, within the scope of this study, the operation of hydroelectric power plants in network operation is of interest, which is the power control mode with frequency response. In this mode, the output power of the unit is adjusted so as to satisfy the set value in the steady state. Additionally, the speed governor responds against frequency deviations as shown in the block diagram illustrated in Fig. 3-12.

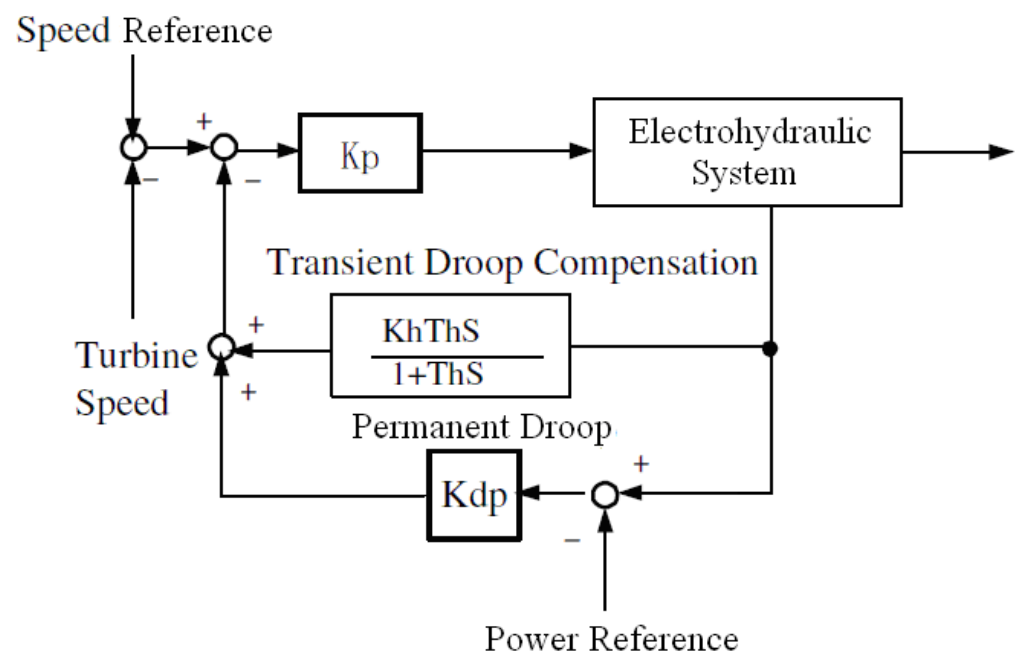

Fig. 3-12: The Block Diagram Representation of a PD governor 


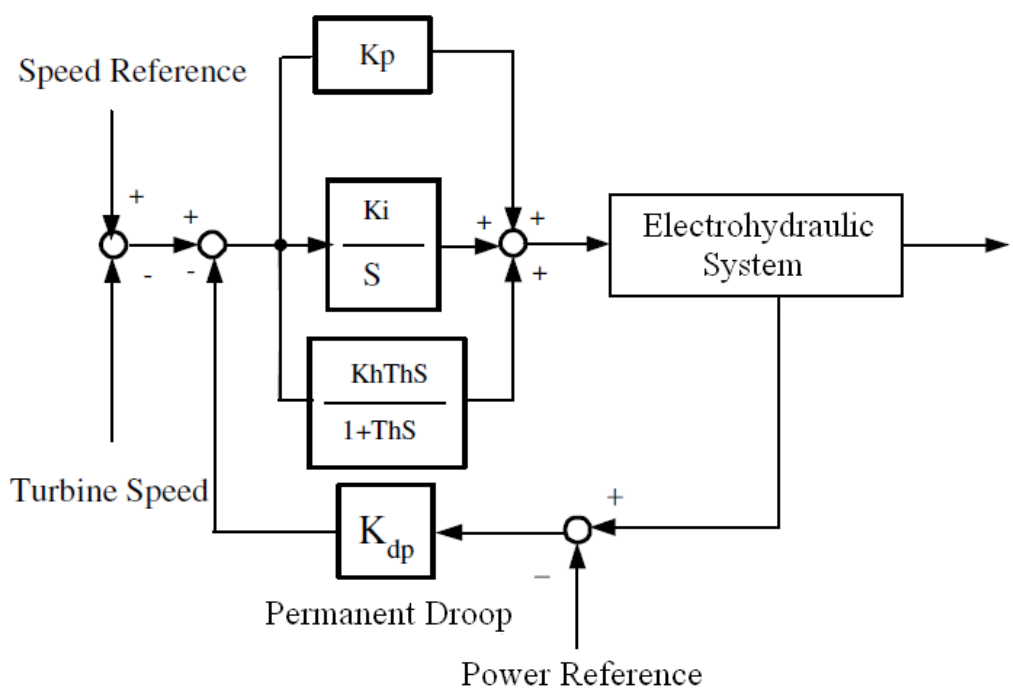

Fig. 3-13: The Block Diagram Representation of a PID Governor

\subsubsection{Generator and Excitation System Modeling}

As stated in Chapter 2, power system swings are classified according to the time frame of interest, in order to simplify the modeling and analysis studies. In traditional power system analysis procedures, the problems regarding the turbine governor systems (i.e. the mechanical part) and the problems regarding the generator, the network and the excitation system (i.e. the electrical part) are dealt separately. However, the specific problem that this study is concentrated on concerns the time frame that is in the overlapping zone of the problems regarding both the mechanical system and the electrical system. In other words, in order to effectively analyze the effects of turbine governors on low frequency inter area oscillations, the electrical part of the generator should also be modeled in adequate accuracy in addition to the mechanical part. 


\subsubsection{Generator Modeling}

\subsection{Park's Transformation and Voltage Equations}

Unlike most of the thermal power plants where the rotor is typically driven at high speed, in most hydroelectric power plants, rotor speed is rather slow due to the big mass of the hydraulic turbines. Hence, the generators utilized in hydroelectric power plants are generally salient pole synchronous generators. Therefore, this type of generators is going to be analyzed throughout this study.

A schematic cross-section of a generator is illustrated in Fig. 3-14. The notation followed in Fig. 3-14 follows the IEEE convention (IEEE Committee Report, 1969). The generator is assumed to have a three-phase stator armature winding (denoted by the letters $\mathrm{A}, \mathrm{B}, \mathrm{C}$ ), a rotor field winding (denoted by F) and two rotor damper windings, one in the d-axis (denoted by $\mathrm{D}$ ) and the other one in the q-axis (denoted by Q). The relative position of the windings and their axes are shown by taking the centre of phase A as the reference.

The following assumptions are made throughout the modeling study [1]:

(i) The three-phase stator winding is symmetrical.

(ii) The capacitance of all the windings can be neglected.

(iii) Each of the distributed windings may be represented by a concentrated winding.

(iv) The change in the inductance of the stator windings due to rotor position is sinusoidal and does not contain higher harmonics.

(v) Hysteresis loss is negligible but the influence of eddy currents can be included in the model of the damper windings.

(vi) In the transient and subtransient states the rotor speed is near synchronous speed $\left(\omega \approx \omega_{\mathrm{s}}\right)$.

(vii) The magnetic circuits are linear (not saturated) and the inductance values do not depend on the current. 

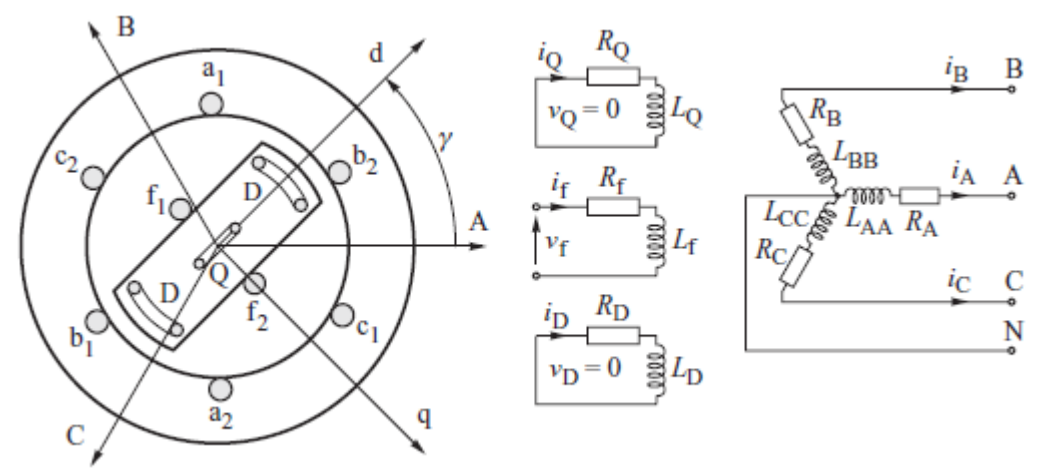

Fig. 3-14: A Schematic Cross Section of a Generator [1]

As can be seen from Fig. 3-14, all generator windings are magnetically coupled which means that the flux induced in each winding depends on the currents in all the other windings [1]. Therefore, the flux linkage equation of a synchronous machine can be written as in (3-24).

$$
\left[\begin{array}{l}
\psi_{A} \\
\psi_{B} \\
\psi_{C} \\
\psi_{f} \\
\psi_{D} \\
\psi_{Q}
\end{array}\right]=\left[\begin{array}{llllll}
L_{A A} & L_{A B} & L_{A C} & L_{A f} & L_{A D} & L_{A Q} \\
L_{B A} & L_{B B} & L_{B C} & L_{B f} & L_{B D} & L_{B Q} \\
L_{C A} & L_{C B} & L_{C C} & L_{C f} & L_{C D} & L_{C Q} \\
L_{f A} & L_{f B} & L_{f C} & L_{f f} & L_{f D} & L_{f Q} \\
L_{D A} & L_{D B} & L_{D C} & L_{D f} & L_{D D} & L_{D Q} \\
L_{Q A} & L_{Q B} & L_{Q C} & L_{Q f} & L_{Q D} & L_{Q Q}
\end{array}\right]\left[\begin{array}{l}
i_{A} \\
i_{B} \\
i_{C} \\
i_{f} \\
i_{D} \\
i_{Q}
\end{array}\right]
$$

The inductance values expressed in (3-24) are not constant but rather a function of position between the stator and the rotor, denoted by $\gamma$.

From Fig. 3-14, it is clear that the self-inductance of each stator phase winding will reach a maximum value whenever the rotor d-axis aligns with the axis of the phase winding because, with the rotor in this position, the reluctance of the flux path is at minimum. This minimum reluctance condition occurs twice during each rotation of the rotor. 
As each of the stator windings is shifted in space relative to the others by $120^{\circ}$, the mutual inductance between each of the stator windings is negative. The magnitude of the inductance is a maximum when the rotor d-axis is midway between the axes of two of the windings.

The mutual inductances between the stator and rotor windings change with rotor position and have a positive maximum value when the axes of a stator winding and the rotor winding align and have the same positive flux direction. When the flux directions are in opposition the value of the inductance is a negative minimum and when the axes are perpendicular the inductance is zero.

The self- and mutual inductances of the rotor windings are constant and do not depend on rotor position. As the $\mathrm{d}$ - and q-axis windings are perpendicular to each other, their mutual inductances are essentially equal to zero [1].

The above mentioned values are generally supplied by generator manufacturers and can be re-calculated using the equations in [1] in extreme cases.

Since the above equations are derived using the flux in the air gap of the generator, it is possible to transform each phasor quantity in the stator reference frame $(\mathrm{A}, \mathrm{B}, \mathrm{C})$ into the $(\mathrm{d}, \mathrm{q})$ reference frame in phasor domain by using simple geometric projections utilizing trigonometric functions of the angle $\gamma$ as in (3-25), so as to maintain the resultant air gap flux constant.

$$
\left[\begin{array}{c}
i_{0 d q} \\
i_{f D Q}
\end{array}\right]=\left[\begin{array}{cc}
W & 0 \\
0 & 1
\end{array}\right]\left[\begin{array}{l}
i_{A B C} \\
i_{f D Q}
\end{array}\right]
$$

where,

$\mathrm{i}_{0 \mathrm{dq}}$ denotes the $3 \mathrm{x} 1$ matrix corresponding to the currents in $0, \mathrm{~d}$, $\mathrm{q}$ axis

$\mathrm{i}_{\mathrm{fDQ}}$ denotes the $3 \times 1$ matrix corresponding to the currents in $\mathrm{f}, \mathrm{D}, \mathrm{Q}$ axis

$\mathrm{W}$ denotes the $3 \times 3$ transformation matrix as in (3-26)

1 denotes the $3 \times 3$ identity matrix 
$\mathrm{i}_{\mathrm{ABC}}$ denotes the $3 \times 1$ matrix corresponding to the currents in $\mathrm{A}, \mathrm{B}, \mathrm{C}$ axis $\mathrm{i}_{\mathrm{fDQ}}$ denotes the $3 \times 1$ matrix corresponding to the currents in $\mathrm{f}, \mathrm{D}, \mathrm{Q}$ axis.

$$
W=\sqrt{\frac{2}{3}}\left[\begin{array}{ccc}
\frac{1}{\sqrt{2}} & \frac{1}{\sqrt{2}} & \frac{1}{\sqrt{2}} \\
\cos \gamma & \cos \left(\gamma-\frac{2}{3} \pi\right) & \cos \left(\gamma+\frac{2}{3} \pi\right) \\
\sin \gamma & \sin \left(\gamma-\frac{2}{3} \pi\right) & \sin \left(\gamma+\frac{2}{3} \pi\right)
\end{array}\right]
$$

It is important to note here that, with this choice of transformation coefficients $\mathrm{W}^{-1}=$ $\mathrm{W}^{\mathrm{T}}$ and a matrix that satisfies this condition is said to be orthogonal. Such an orthogonal transformation between frames is necessary in order to ensure that the power calculated in the $(\mathrm{A}, \mathrm{B}, \mathrm{C})$ and in the $(\mathrm{d}, \mathrm{q})$ reference frames is identical. In this case, the transformation is said to be power invariant which is an important property.

The resultant fictitious perpendicular windings representing the synchronous generator in (dq0) frame is illustrated in Fig. 3-15.

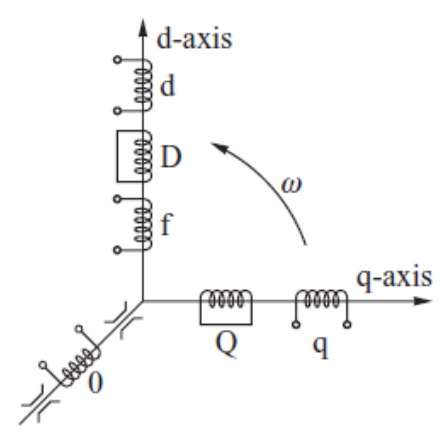

Fig. 3-15: The Representation of the Synchronous Generator in dq0 Frame

The voltage equations of the generator illustrated in Fig. 3-15 can be derived by the basic Kirchoff's Voltage Law, considering both the inductances and the resistances of the windings. 
In order to derive the fluxes through the windings, the inverse transformation of (325 ) is used and (3-27) is found, where both the transformation matrix W (since the position of the rotor with respect to the stator, that is $\gamma$, varies with time) and the currents are functions of time.

$$
\left[\begin{array}{c}
\psi_{0 d q} \\
\psi_{f D Q}
\end{array}\right]=\left[\begin{array}{cc}
W L_{S} W^{-1} & W L_{S R} \\
L_{S R}{ }^{T} W^{-1} & L_{R}
\end{array}\right]\left[\begin{array}{c}
i_{0 d q} \\
i_{f D Q}
\end{array}\right]
$$

Explicitly, (3-27) can be written as in (3-28) where $k=\sqrt{\frac{3}{2}}$. In (3-28), the rotor and stator axis are stationary with respect to each other and hence although the fluxes and currents are functions of time, the inductances are constant. (3-28) is called Park's Equation and it is widely used in dynamical studies regarding synchronous machines.

$$
\left[\begin{array}{l}
\psi_{0} \\
\psi_{d} \\
\psi_{q} \\
\psi_{f} \\
\psi_{D} \\
\psi_{Q}
\end{array}\right]=\left[\begin{array}{cccccc}
L_{0} & & & & & \\
& L_{d} & & k M_{f} & k M_{D} & \\
& & L_{q} & & & k M_{Q} \\
& k M_{f} & & L_{f} & L_{f D} & \\
& k M_{D} & & L_{f D} & L_{D} & \\
& & k M_{Q} & & & L_{Q}
\end{array}\right]\left[\begin{array}{c}
i_{0} \\
i_{d} \\
i_{q} \\
i_{f} \\
i_{D} \\
i_{Q}
\end{array}\right]
$$

By writing the KCL in the generator circuit illustrated in Fig. 3-15, (3-29) is achieved.

$$
\left[\begin{array}{c}
v_{0 d q} \\
v_{f D Q}
\end{array}\right]=-\left[\begin{array}{cc}
W & 0 \\
0 & I
\end{array}\right]\left[\begin{array}{cc}
R_{A B C} & 0 \\
0 & R_{f D Q}
\end{array}\right]\left[\begin{array}{cc}
W^{-1} & 0 \\
0 & I
\end{array}\right]\left[\begin{array}{c}
i_{0 d q} \\
i_{f D Q}
\end{array}\right]-\left[\begin{array}{cc}
W & 0 \\
0 & I
\end{array}\right] \frac{d}{d t}\left\{\left[\begin{array}{cc}
W^{-1} & 0 \\
0 & I
\end{array}\right]\left[\begin{array}{l}
\psi_{0 d q} \\
\psi_{f D Q}
\end{array}\right]\right\}
$$

Since both the transformation matrix $\mathrm{W}$ and the flux linkages are functions of time, two components of induced voltage on windings is achieved; namely the rotational emfs (the terms multiplied by machine speed $\mathrm{w}$ in (3-30))and the transformer emfs 
(the $\dot{\psi}$ terms in (3-30)). It is important to note here that the d-axis rotational emf is induced by the q-axis flux, whereas the q-axis rotational emf is induced by the d-axis flux. The signs are a result of the assumed direction. The armature emfs proportional to the rate of change of the flux, are referred to as the transformer emfs. Those components of voltage are due to the time variation of currents in coils on the same axis as the one where the voltage is being induced.

$$
\begin{aligned}
& v_{0}=-R i_{0}-\dot{\psi}_{0} \\
& v_{d}=-R i_{d}-\dot{\psi}_{d}-w \psi_{q} \\
& v_{q}=-R i_{q}-\dot{\psi}_{q}-w \psi_{d} \\
& v_{f}=R_{f} i_{f}+\dot{\psi}_{f} \\
& 0=R_{D} i_{D}+\dot{\psi}_{D} \\
& 0=R_{Q} i_{Q}+\dot{\psi}_{Q}
\end{aligned}
$$

\subsection{Analysis Assumptions and Synchronous Generator Equations}

As stated before, in power system analysis, the specific problems accociated with different time frames are analyzed by making the reasonable assumptions regarding the time frame of interest. Similarly, while analyzing the dynamic behaviour of a synchronous generator, several assumptions are made to simplify the analysis. The dynamic behaviour of the machine is analyzed through three time frames those are the steady state, the transient state and the subtransient state.

In the steady state, it is assumed that the armature flux penetrates through the rotor circuit, which means that no flux change occurs in the rotor circuit and no opposing current in the field winding and in the damper windings is induced (i.e. all transient currents in the field winding and in the damper windings have died out). Therefore, the field and damper winding currents are constant and the armature is simply modeled by the synchronous inductance $\mathrm{L}_{\mathrm{d}}$ in the direct (d) axis and $\mathrm{L}_{\mathrm{q}}$ in the quadrature (q) axis. The generator is modeled by the equations in (3-31). 


$$
\begin{aligned}
& v_{d}=-R_{d} i_{d}-X_{q} i_{q} \\
& v_{q}=-R_{q} i_{q}+X_{d} i_{d}+w k M_{f} i_{f}
\end{aligned}
$$

In the transient state, it is assumed that the armature flux penetrates through the damper circuits which means that no opposing current in the damper windings is induced (i.e. all transient currents in the damper windings have died out). However, the field winding screens the rotor body from the armature flux via induced opposing currents. Hence, the damper circuits are no longer effective and can be removed from the model, while the field winding is modelled by short-circuiting the field winding and setting its resistance to zero. This effectively represents the current changes that would occur in the field winding in order to maintain the flux linkage of this winding constant, the definition of the transient state [1].

Since the dampers are ineffective, the synchronous inductances and impedances seen from the armature are as in (3-32).

$$
\begin{aligned}
& L_{d}^{\prime}=L_{d}-\frac{L_{f d}{ }^{2}}{L_{f}} \\
& X_{d}^{\prime}=w L_{d}^{\prime} \\
& L_{q}^{\prime}=L_{q} \\
& X_{q}^{\prime}=w L_{q}^{\prime}=w L_{q}
\end{aligned}
$$

Mostly the synchronous generators are modeled in transient state by the decay time constants of the induced field current since those time constants are practically easy to determine via tests by the generator manufacturers. These time constants depend on whether the d-axis armature coil is open circuit or short circuit. Therefore, these time constants are named according to the condition of the armature coil as $T_{d o}{ }^{\prime}(\mathrm{d}$ axis transient open circuit time constant) and as $T_{d}^{\prime}$ (d axis transient short circuit time constant). As the name implies, these time constants are determined by open circuiting and short circuiting the armature winding as formulated in (3-33). 


$$
\begin{aligned}
& T_{d o}{ }^{\prime}=\frac{L_{f}}{R_{f}} \\
& T_{d}{ }^{\prime}=\left(L_{f}-\frac{L_{f d}^{2}}{L_{d}}\right) \frac{1}{R_{f}}=T_{d o} \cdot \frac{L_{d}{ }^{\prime}}{L_{d}}
\end{aligned}
$$

The generator is modeled by the equations as in (3-34).

$$
\begin{aligned}
& v_{d}=-R_{d} i_{d}-X_{q}^{\prime} i_{q} \\
& v_{q}=-R_{q} i_{q}+X_{d}^{\prime} i_{d}+e_{q}^{\prime} \\
& v_{f}=\dot{\psi}_{f}+\frac{R_{f}}{L_{f}} \psi_{f}-R_{f} \frac{k M_{f}}{L_{f}} i_{d}
\end{aligned}
$$

where $e_{q}^{\prime}=\frac{k M_{f}}{L_{f}} \psi_{f}$ is called the quadrature axis transient emf since $\psi_{\mathrm{f}}$ is no longer equal to the q axis steady state emf.

In the subtransient state, the rotor damper coils screens both the field winding and the rotor body from changes in the armature flux. Since the subtransient period is quite small, it is assumed that the field flux linkages $\psi_{\mathrm{f}}$ remain constant during this period while the damper winding flux linkages are constant immediately after the fault or disturbance. However, due to the small time constant of the damper windings, the subtransient currents decay with time as the generator moves towards the transient state [1]. The equations modeling the generator in the subtransient state are as in (3$35)$.

$$
\begin{aligned}
& v_{q}=-R i_{q}+X_{d}^{\prime \prime} i_{d}+e_{q}^{\prime \prime} \\
& v_{d}=-R i_{d}-X_{q}^{\prime \prime} i_{q}+e_{d}^{\prime \prime}
\end{aligned}
$$

where, $e_{q}^{\prime \prime}=w\left(\left(\frac{k M_{f} L_{D}-k M_{D} L_{f D}}{L_{f} L_{D}-L_{f D}^{2}}\right) \psi_{f}+\left(\frac{k M_{D} L_{f}-k M_{f} L_{f D}}{L_{f} L_{D}-L_{f D}^{2}}\right) \psi_{D}\right)$. 
In this study, in addition to the equations listed from (3-24) to (3-35), the swing equation and the electrical power equations are also included in the generator model. Hence the set of differential equations in $(d, q)$ reference frame in per unit quantities are as listed in (3-36). The quantities can be converted to (ABC) reference frame by inverse transformation. However, since the transformation is power invariant, the mentioned inverse transformation is applied seldomly.

$$
\begin{aligned}
& {\left[\begin{array}{l}
v_{d} \\
v_{q}
\end{array}\right]=\left[\begin{array}{l}
e_{d}^{\prime \prime} \\
e_{q}^{\prime \prime}
\end{array}\right]-\left[\begin{array}{cc}
R_{d} & X_{q}^{\prime \prime} \\
-X_{d}^{\prime \prime} & R_{d}
\end{array}\right]\left[\begin{array}{l}
i_{d} \\
i_{q}
\end{array}\right]} \\
& P_{e}=e_{q}^{\prime \prime} i_{q}+e_{d}^{\prime \prime} i_{d}+\left(X_{d}^{\prime \prime}-X_{q}^{\prime \prime}\right) i_{d} i_{q} \\
& 2 H \Delta \dot{w}=P_{m}-P_{e}-D \Delta w \\
& \dot{\delta}=\Delta w \\
& T_{d o}^{\prime} \ddot{e}_{q}^{\prime}=e_{f}-\dot{e}_{q}+i_{d}\left(X_{d}-X_{d}^{\prime \prime}\right) \\
& T_{d o}^{\prime \prime} \dot{e}_{q}^{\prime \prime}=e_{q}^{\prime}-e_{q}^{\prime \prime}+i_{d}\left(X_{d}^{\prime}-X_{d}^{\prime \prime}\right) \\
& T_{q o}^{\prime \prime} \dot{e}_{d}^{\prime}=e_{d}^{\prime}-e_{d}^{\prime \prime}-i_{q}\left(X_{q}^{\prime}-X_{q}^{\prime \prime}\right)
\end{aligned}
$$

It is important to note here that changes in $\mathrm{P}_{\mathrm{m}}$ should be calculated from the models describing the dynamical behavior of turbines and governors as discussed in 3.3.2. whereas the changes in ef should be calculated from the models describing the dynamical behaviour of excitation systems as discussed in sections 3.3.3.2. and 3.3.3.3.

The model used in (3-36) is a widely accepted model that is considered to be sufficiently accurate in analyzing electromechanical dynamics, which is the focus of this study. The assumptions made in this model are neglecting the effect of rotor body eddy currents on $\mathrm{q}$ axis, hence $X_{q}^{\prime}=X_{q}$ in the model, and ignoring the damping component of power due to friction and windage which is quite small as compared with the damping power arising from the induced currents in the damper windings. 
For small signal studies analyzing the oscillatory stability of the generator, the model proposed in [15] is also widely used, which is a linearization of the simplified version of the model in proposed in (3-36) at specific operating points.

For more detailed studies regarding the generator dynamics such as excitation system tuning, the saturation characteristics of the generator should also be taken into account; however, the details to this extend is beyond the scope of this study and hence will not be considered in the models.

\subsubsection{Excitation System Modeling}

The principal aim of an excitation system is to control the field current of a synchronous generator so as to control either the terminal voltage or the reactive power or the power factor of the generator at the specified value. In practice, a wide variety of excitation systems realizing the above objective are being utilized.

In particular, an excitation system consists of the Automatic Voltage Controller $(\mathrm{AVR})$ and the exciter which maintains the field current. In literature, there are three main types of excitation systems those are; DC exciters, AC rotating exciters and static exciters. Detailed information regarding different types of excitation systems can be found in IEEE Committee Reports on excitation systems (IEEE Committee Report 1968, 1973a, 1973b, 1973c, 1981, 1992). In modern excitation systems, static excitors, which are principally three phase controlled bridge rectifiers, are being utilized. Hence, this type of excitation systems will be considered in this study.

The block diagram of the computer model of IEEE Type ST1A excitation system model [31], representing the static excitation system utilized in this study is illustrated in Fig. 3-16. 


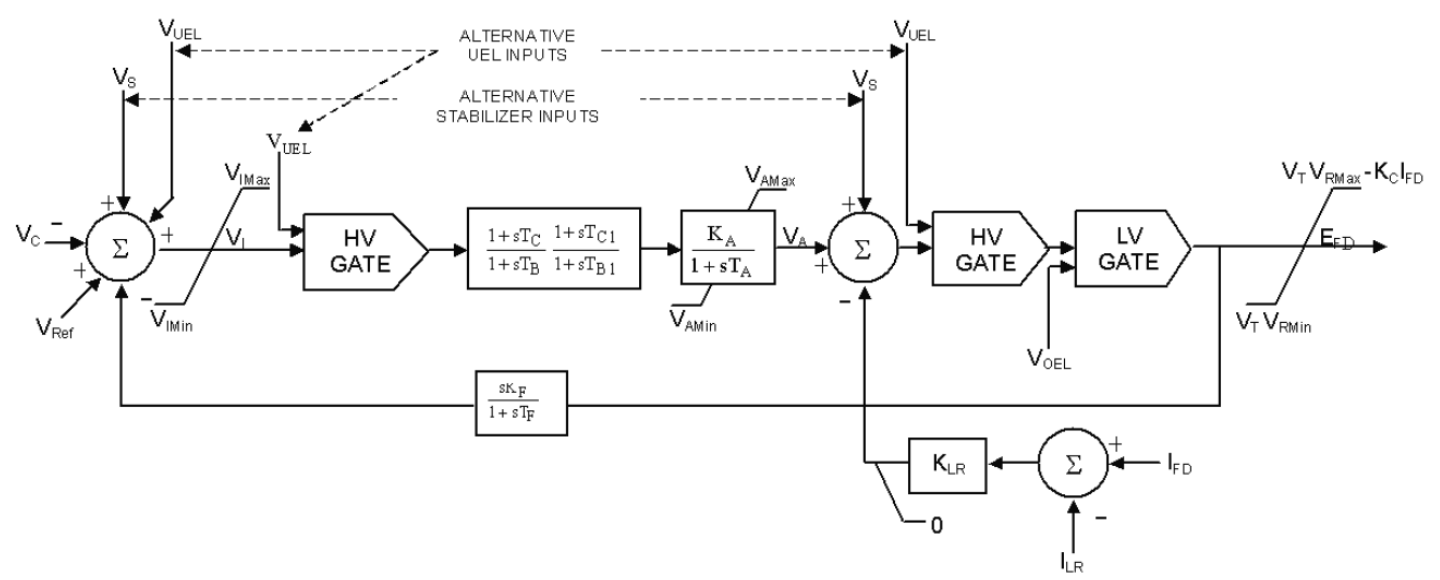

Fig. 3-16: IEEE Type ST1A Excitation System Model [31]

In the model, voltage regulator gain and any inherent excitation system time constants are represented by $\mathrm{K}_{\mathrm{A}}$ and $\mathrm{T}_{\mathrm{A}}$, respectively. Transient gain reduction can be implemented either in the forward path via time constants, $T_{B}$ and $T_{C}$ (in which case $\mathrm{K}_{\mathrm{F}}$ would normally be set to zero), or in the feedback path by suitable choice of rate feedback parameters, $\mathrm{K}_{\mathrm{F}}$ and $\mathrm{T}_{\mathrm{F}}[31]$.

As stated in 3.3.3.1., this excitation system model is interfaced with the dynamical model of the generator in dynamical studies.

\subsubsection{PSS Modeling}

The principal aim of a PSS is helping to damp generator rotor oscillations by providing an additional signal which produces a torque component that is in phase with the rotor speed deviations. As it is the case for excitation systems, in practice, a wide variety of excitation systems realizing the above objective are being utilized depending on the requirement of the specific generator.

In practice, there are basically three widely used types of PSSs; those are speed based stabilizers $(\mathrm{Dw})$, frequency based stabilizers $(\Delta \mathrm{f})$, power based stabilizers $(\Delta \mathrm{P})$ and 
integral of accelerating power stabilizers $(\Delta \mathrm{Pw})$. The first mentioned three types of of PSSs all have some drawbacks as discussed in [20] and the integral of accelerating power type stabilizers, examples of which are the IEEE Standard PSS2A and PSS2B, are the de-facto modern industrial applications. Since the scope of this study is investigating the effects of governor settings of hydroelectric power plants to low frequency inter area oscillations, the concerns about excitation systems regarding damping of inter area modes are not focused in this study and instead left as a future work. Therefore, a detailed PSS tuning study will not be performed in this study, however, a block diagram of IEEE Standard PSS2A is illustrated in Fig. 3-17, for the sake of completeness.

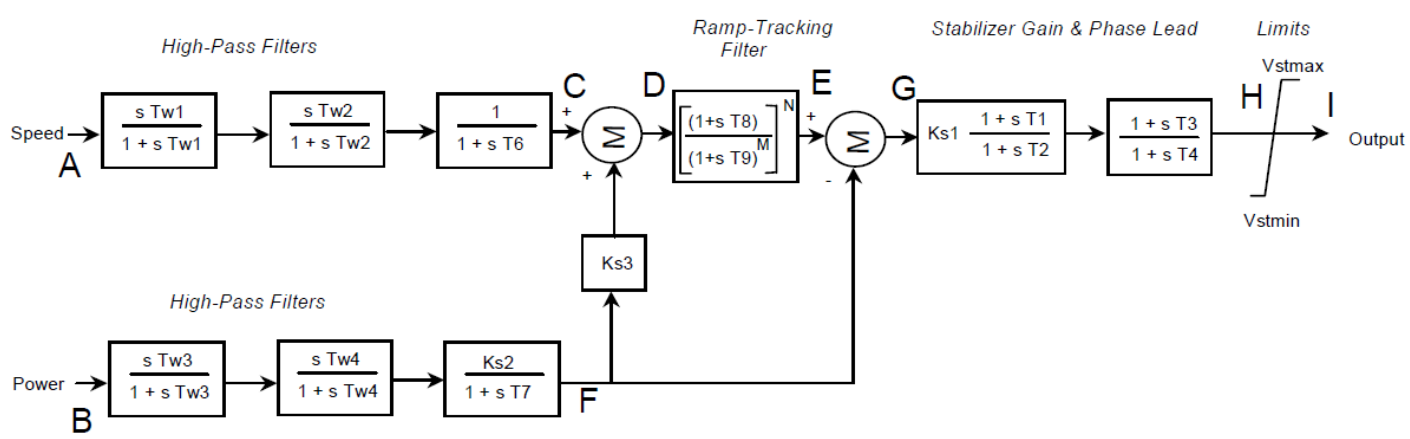

Fig. 3-17: The Block Diagram of IEEE Standard PSS2A Integral of Accelerating Power Stabilizer [20]

\subsection{Network Modeling for Oscillatory Stability Studies}

In order to assess the oscillatory stability of the general network rather than a single generator, a linearized network of the grid should be formed.

The power flow equations in the steady state of the system are well known and utilized. Similar derivation procedure of power flow equations is also valid in dynamical studies by just transforming the equations in (dq0) frame and utilizing the generator model as explained in 3.3.3.1. and expressed by the set of equations as in 
(3-36). A further assumption simplifying the analysis is to assume the loads, including also the ones in the generator terminals, as constant impedances and obtain an equivalent network consisting of generators and impedances.

Hence, the power injected to the equivalent network from the $\mathrm{i}^{\text {th }}$ generator is expressed as in (3-37).

$$
\begin{aligned}
P_{i}= & e_{d_{i}}^{\prime \prime} \sum_{j=1}^{n}\left[\left(B_{i j} \cos \delta_{i j}-G_{i j} \sin \delta_{i j}\right) e_{q_{j}}^{\prime \prime}+\left(B_{i j} \sin \delta_{i j}-G_{i j} \cos \delta_{i j}\right) e_{d_{j}}^{\prime \prime}\right]+ \\
& e_{q_{i}}^{\prime \prime} \sum_{j=1}^{n}\left[\left(B_{i j} \sin \delta_{i j}-G_{i j} \cos \delta_{i j}\right) e_{q_{j}}^{\prime \prime}-\left(B_{i j} \cos \delta_{i j}-G_{i j} \sin \delta_{i j}\right) e_{d_{j}}^{\prime \prime}\right]
\end{aligned}
$$

Therefore, linearizing (3-37) gives the network power flow equation in (3-38).

$$
[\Delta \boldsymbol{P}]=\left[\begin{array}{lll}
\frac{\partial \boldsymbol{P}}{\partial \boldsymbol{\delta}_{n-1}} & \frac{\partial \boldsymbol{P}}{\partial \mathbf{e}_{q}^{\prime \prime}} & \frac{\partial \boldsymbol{P}}{\partial \mathbf{e}_{d}^{\prime \prime}}
\end{array}\right]\left[\begin{array}{c}
\boldsymbol{\delta}_{n-1} \\
\mathbf{e}_{q}^{\prime \prime} \\
\mathbf{e}_{d}^{\prime \prime}
\end{array}\right]
$$

where,

$\Delta \mathbf{P}$ denotes the $(\mathrm{nx} 1)$ vector representing the power difference at the generator terminals

$\mathbf{P}$ denotes the (nx1) vector representing the injected power of each generator

$\boldsymbol{\delta}$ denotes the $((\mathrm{n}-1) \times 1)$ vector representing the load angle of each generator with respect to the reference machine

$\mathbf{e}_{\mathbf{q}}{ }^{\prime}$ denotes the (nx1) vector representing the subtransient emf of each generator in $\mathrm{q}$ axis

$\mathbf{e}_{\mathbf{d}}$ " denotes the (nx1) vector representing the subtransient emf of each generator in $\mathrm{d}$ axis

Similarly, the transformed voltage equations in the (dq0) reference frame can be written as in (3-39). 


$$
\begin{aligned}
& v_{q_{i}}=e_{q_{i}}^{\prime \prime}+X_{d_{i}}^{\prime \prime} \sum_{j=1}^{n}\left[\left(B_{i j} \cos \delta_{i j}-G_{i j} \sin \delta_{i j}\right) e_{q_{j}}^{\prime \prime}+\left(B_{i j} \sin \delta_{i j}+G_{i j} \cos \delta_{i j}\right) e_{d_{j}}^{\prime \prime}\right] \\
& v_{d_{i}}=e_{d_{i}}^{\prime \prime}-X_{q_{i}}^{\prime \prime} \sum_{j=1}^{n}\left[\left(B_{i j} \sin \delta_{i j}+G_{i j} \sin \delta_{i j}\right) e_{q_{j}}^{\prime \prime}-\left(B_{i j} \cos \delta_{i j}-G_{i j} \sin \delta_{i j}\right) e_{d_{j}}^{\prime \prime}\right] \\
& v_{i}=\sqrt{v_{q_{i}}{ }^{2}+v_{d_{i}}^{2}}
\end{aligned}
$$

Therefore, linearizing (3-39) gives the network voltage equation in (3-40).

$$
[\Delta \mathbf{V}]=\left[\begin{array}{lll}
\frac{\partial \mathbf{V}}{\partial \boldsymbol{\delta}_{n-1}} & \frac{\partial \mathbf{V}}{\partial \mathbf{e}_{q}^{\prime \prime}} & \frac{\partial \mathbf{V}}{\partial \mathbf{e}_{d}^{\prime \prime}}
\end{array}\right]\left[\begin{array}{c}
\boldsymbol{\delta}_{n-1} \\
\mathbf{e}_{q}^{\prime \prime} \\
\mathbf{e}_{d}^{\prime \prime}
\end{array}\right]
$$

where,

$\Delta \mathbf{V}$ denotes the (nx1) vector representing the voltage magnitude difference at the generator terminals

$\mathbf{V}$ denotes the (nx1) vector representing the magnitude of the terminal voltage of each generator

$\boldsymbol{\delta}$ denotes the $((\mathrm{n}-1) \times 1)$ vector representing the load angle of each generator with respect to the reference machine

$\mathbf{e}_{\mathbf{q}}$ " denotes the $(\mathrm{nx} 1)$ vector representing the subtransient emf of each generator in $\mathrm{q}$ axis

$\mathbf{e}_{\mathbf{d}}$ " denotes the (nx1) vector representing the subtransient emf of each generator in $\mathrm{d}$ axis

The linearization of (3-36) ends up with (3-41), i.e., the linearized generator differential equations to be used in the small signal model.

$$
\begin{aligned}
& \dot{\boldsymbol{\delta}}_{n-1}=\mathbf{I}_{n-1} \Delta \mathbf{w} \\
& 2 \mathbf{H} \Delta \dot{\mathbf{w}}=-\Delta \mathbf{P}-\mathbf{D} \Delta \mathbf{w} \\
& \mathbf{T}_{d o}^{\prime} \dot{\mathbf{e}}_{q}^{\prime}=-\Delta \mathbf{e}_{q}^{\prime}+\Delta \mathbf{X}_{d}^{\prime} \Delta \mathbf{i}_{d}+\Delta \mathbf{e}_{f} \\
& \mathbf{T}_{d o}^{\prime \prime} \dot{\mathbf{e}}_{q}^{\prime \prime}=-\Delta \mathbf{e}_{q}^{\prime}-\Delta \mathbf{e}_{q}^{\prime \prime}+\Delta \mathbf{X}_{d}^{\prime \prime} \Delta \mathbf{i}_{d} \\
& \mathbf{T}_{q o}^{\prime \prime} \dot{\mathbf{e}}_{d}^{\prime \prime}=-\Delta \mathbf{e}_{d}^{\prime \prime}-\Delta \mathbf{X}_{q}^{\prime \prime} \Delta \mathbf{i}_{q}
\end{aligned}
$$


where, $\Delta \mathbf{e}_{\mathrm{q}}{ }^{\prime}, \Delta \mathbf{e}_{\mathrm{q}}{ }^{\prime}, \Delta \mathbf{e}_{\mathrm{d}}{ }^{\prime}, \Delta \mathbf{i}_{\mathrm{q}}, \Delta \mathbf{P}, \Delta \mathbf{w}$ are the (nx1) vectors corresponding to the generator variable of interest, $\Delta \boldsymbol{\delta}_{\mathrm{n}-1}$ is the ((n-1)x1) vector corresponding to the vector of machine angles with respect to the reference generator, $\mathbf{T}_{\mathrm{qo}}{ }^{\prime}, \mathbf{T}_{\mathrm{do}}, \mathbf{H}, \mathbf{D}$, $\Delta \mathbf{X}_{\mathrm{d}}{ }^{\prime}, \Delta \mathbf{X}_{\mathrm{d}}{ }^{\prime}$ and $\Delta \mathbf{X}_{\mathrm{q}}{ }^{\prime}$ are (nxn) diagonal matrices such that $\Delta \mathbf{X}_{d}^{\prime}=\operatorname{diag}\left(X_{d i}-X_{d i}^{\prime}\right)$, $\Delta \mathbf{X}_{d}^{\prime \prime}=\operatorname{diag}\left(X_{d i}^{\prime}-X_{d i}^{\prime \prime}\right)$ and $\Delta \mathbf{X}_{q}^{\prime \prime}=\operatorname{diag}\left(X_{q i}^{\prime}-X_{q i}^{\prime \prime}\right)$.

Since the actual network is regulated, it is important to include the effects of voltage regulators and PSSs in the overall system equations. In order to simplify the analysis, the reduced AVR and PSS model proposed in [1] can be utilized as illustrated in Fig. $3-18$.

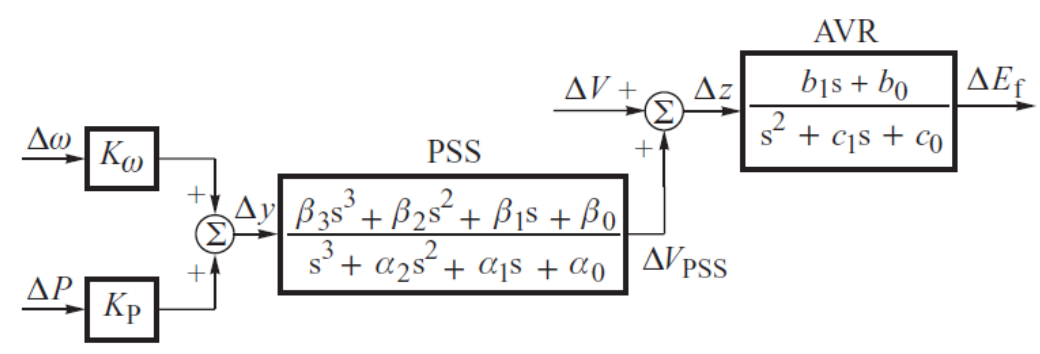

Fig. 3-18: The Reduced AVR and PSS Model to be Used in Network Small Signal Analysis [1]

The expression of the model proposed in Fig. 3-18 in state space, introduces five additional state variables denoted by $\mathrm{X}_{1}$ to $\mathrm{X}_{5}$, to the ones in previous equations from (3-37) to (3-41) and the linearized network model in (3-42) is achieved. 


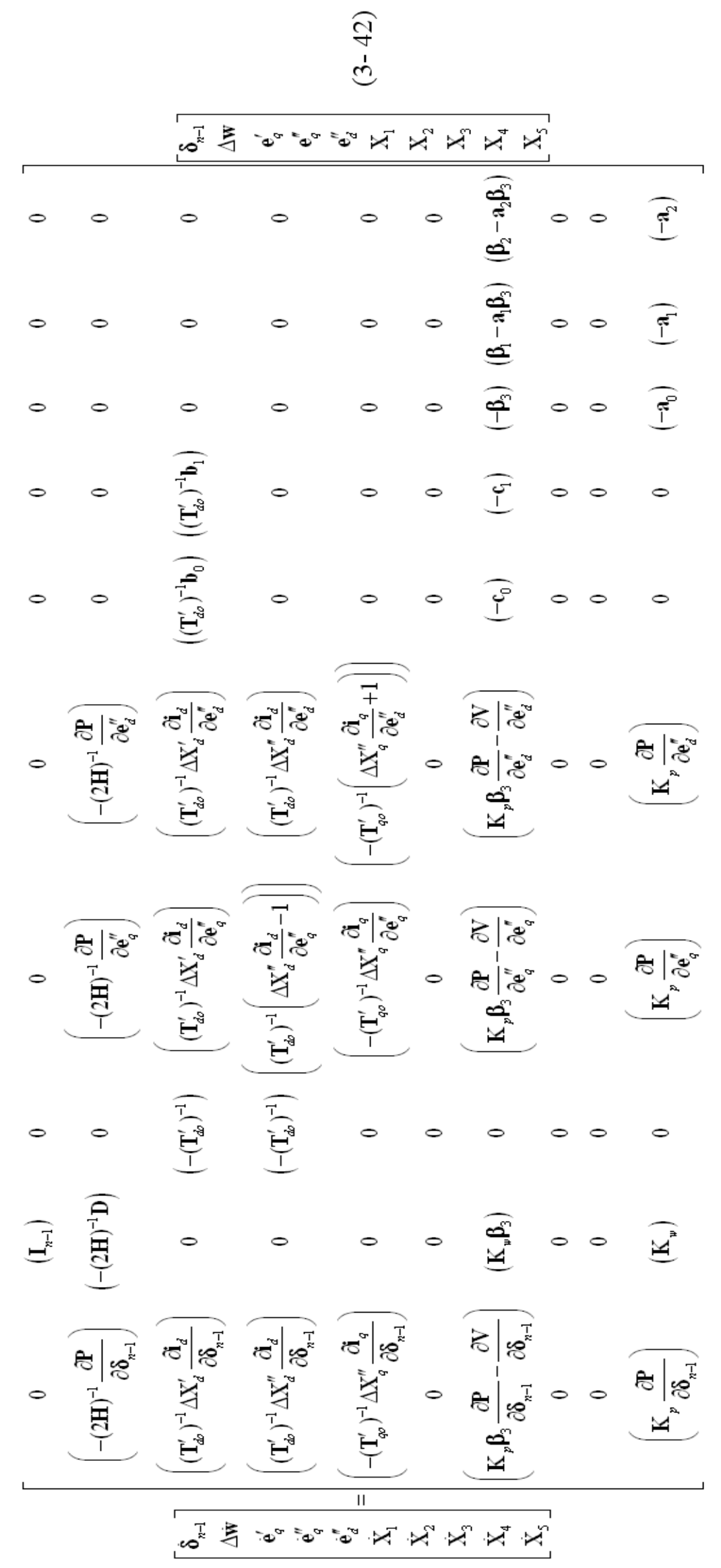




\section{CHAPTER 4}

\section{SIMULATION RESULTS}

\subsection{Introduction}

The main motivation of this part of the study is to propose controller settings for the mechanical speed-power control structures of the considerable size hydroelectric power plants those are mentioned in [6], via performing reliable simulations, the results of which could be applicable on site.

Therefore, in this chapter of the thesis, in order to effectively model a hydroelectric unit so as to analyze and determine its behavior in case of dynamically varying system conditions, first the mathematical model of the unit is formed as explained in Chapter 3, according to the related dynamical data of the hydroelectric power plants that has been surveyed by both TEIAS and the Power Systems Department of TUBITAK UZAY. As stated in Chapter 3, every hydroelectric power plant is designed according to the specific geographical structure of the site. Hence, no off the shelf universal nonlinear mathematical model satisfactorily representing the dynamical behavior of the plant is available which makes the model validation study a key part of the modeling process. The simulations mentioned throughout this chapter are performed by using MATLAB Simulink and DIgSILENT.

Once it is concluded that the computer simulation model satisfactorily represents the dynamical behavior of the hydroelectric unit by the model verification study, the model parameter tuning study is performed as will be explained in the proceeding 
parts of this chapter. As stated in Chapter 2, the main focus of the tuning study is on the effect of the speed control loop in the expected frequency range of possible inter area oscillations after the synchronous interconnection of Turkish Power System with ENTSO-E CESA (former UCTE) System. While performing the parameter tuning study, it is assumed that the single machine is connected to a very large power system which will be modeled by an infinite bus with constant frequency.

After a suitable set of parameters is achieved using a single machine infinite busbar model, the effectiveness of the proposed parameter set for network operation is assessed for multi machine case by using Kundur's well known four machine, two area network [14]. Note that the two area four machine model proposed in [14] is simple enough to observe the effect of a component of a single machine on the multi machine power system without performing simulations by utilizing a bulk power system data and yet powerful enough to get a rough idea about the behavior of the plant under dynamically varying (i.e., oscillatory) system conditions. By the results of both the tuning study and the multi machine simulation study, the locally optimized parameter set is concluded to be suitable for network operation. In addition, this approach significantly reduces the complexity of the global system simulations.

Finally, the overall effect of the retuned hydroelectric power plants in the priority list is shown by time domain analysis using the dynamical data provided by the University of Rostock, which is comprised of the representative dynamical model data of ENTSO-E CESA network and complete dynamical data of Turkish Power System, which has been surveyed by mostly the Power Systems Department of TUBITAK UZAY and partially by TEIAS. In this part of the study, DIgSILENT Power Factory program is used to simulate the entire system which also utilizes the state space representation of the system as expressed in (3-42). 


\subsection{Str Hydroelectric Power Plant Governor Retuning Study}

A successful dynamical hydroelectric unit model requires knowledge on a wide variety of different disciplines of engineering from fluid mechanics to electromechanical energy conversion. Hence, in the dynamical studies, some simplifying assumptions like the superposition principal for LTI systems should be made in order to analyze the effect of a specific phenomenon.

As stated before, the most important assumption made in this study is the time frame of interest. Since the dynamics caused by the excitation system decay so fast in comparison with those of the mechanical system, it is assumed in governor tuning studies that the generator and excitation system dynamics are ineffective which means that the dynamics regarding the mechanical power of the turbine can be observed from the electrical power output of the unit measured from the generator terminals. In other words, comparatively fast electrical transients inherent in the generator are ignored while performing mechanical system modeling. However, in multi machine case simulations and in global system studies including sensitivity analysis, the electrical transients in the generators are also modeled together with the mechanical systems.

\subsubsection{Governor Tuning Study}

Generally there are multiple identical units in a hydroelectric power plant. Similarly, in Sir Hydroelectric Power Plant, there are three Francis Type hydroelectric turbines each rated at 94.5 MW. Since the same construction and electromechanical equipment is utilized in all of the identical units, both the site tests and the parameter tuning study can be performed in a single unit. Afterwards, it will be assumed that the proposed settings are valid for all the identical units. 
The major system-wide constraints, those are taken into account while tuning considering the ENTSO-E CESA interconnection of Turkish Power System, are listed as follows:

(i) The stability of frequency control in case of islanding from ENTSO-ECESA, which may occur due to the action of the protection system, should be ensured.

(ii) The Turkish Power System as a control area, should be capable of activating the sufficient primary control reserve according to the ENTSOE-CESA requirements (approximately $300 \mathrm{MW}$ in $30 \mathrm{~s}$ in case of $200 \mathrm{mHz}$ frequency deviations), which means that the primary control reserve should not exceed the necessary value (i.e., the discrepancy in the tie line power should not exceed the Transmission Reliability Margin of Net Transfer Capacity should not be exceeded).

(iii) The speed governing structures should not have negative effects on the damping of inter-area oscillations.

The reflections of the above listed system-wide expectations regarding interconnected operation constitute the expectations from a considerable size individual hydroelectric unit from the viewpoint of the TSO, which can be stated as follows:

(i) As stated in [5], due to the high percentage of hydroelectric power plants in Turkey, the hydroelectric power plants should operate so as be stable in island mode even if they operate interconnected to the grid.

(ii) For the step response tests, a settling time comparable with 30 seconds should be achieved .

(iii) The turbine governor systems of the hydroelectric power plants should not deteriorate the damping performance of the system for low frequency inter area power swings. 


\subsubsection{Modeling of a Unit of Sur Hydroelectric Power Plant}

In general, any synchronous generating unit can be modeled by the superposition of two dependent sub-models, those are:

(i) The turbine-governor model, which concerns the mechanical dynamics of the generating unit, maintaining the mechanical power input to the generator shaft.

(ii) The generator and excitation system model maintaining the voltage at the generator terminals.

The mathematical preliminaries and set of differential equations concerning both sub-models are explained in Chapter 3 in detail. As explained in the part 3.3.2.1., the mechanical power output of the turbine is dependent on the hydraulic pressure and flow, which is provided by the turbine manufacturer as a family of curves, commonly referred to as the "Shell Curve" of the turbine. By utilizing the Shell Curve for the turbine of the related unit of Sir Hydroelectric Power Plant, the mechanical power of the unit is expressed as a function of hydraulic head and flow as illustrated in Fig. 38 in the previous chapter.

Another important nonlinear function utilized in the nonlinear turbine model proposed in the part 3.3.2.1., is the relation between the effective flow area and the guide vane (wicket gate) opening. This relation is generally not provided by the turbine manufacturers and hence can only be obtained by site tests by following the iterative procedure below.

(i) In the site tests, the governor of the turbine is set to "Constant Opening Control" mode which provides the guide vane position (the percentage opening of the wicket gates) at the set value regardless of the frequency deviations.

(ii) In the site tests, at several guide vane (wicket gate) opening values beginning with the no load opening, by steps of $10 \%$ guide vane position 
changes, at each operating point, the output power of the unit is recorded at the steady state.

(iii) The relationship relating mechanical power to flow and head is reexpressed by relating flow to mechanical power and head.

(iv) By iterating through the selected starting point on the function relating mechanical power to flow and head, the flow value corresponding to the mechanical power and hydraulic head is found.

(v) By using the relation (3-12), the effective flow area is found.

The effective flow area of a unit of Sir Hydroelectric Power Plant expressed as a function of guide vane opening is as illustrated in Fig. 3-6 in the previous chapter. The other time constants and differential equations regarding the nonlinear dynamical model are obtained in a straight forward manner by using the equations from (3-12) to (3-19) as explained in the previous chapter.

The governor of the unit is observed to be a variation of PD type governor that was illustrated in Fig. 3-12, the settings and the exact block diagram of which are surveyed on site.

In addition, the generator and excitation system models of the unit are formed according to the surveyed data as in equation (3-36), which is explained in the previous chapter.

The model verification study is realized by comparing the simulation outputs of the dynamical simulation model of the unit with the measurements taken in the site tests. In the site tests, one of the identical units is loaded and unloaded by step power set point changes, when the speed control loop of the governor is active. Afterwards, the wicket gate opening (guide vane position) signal and active power output signal of the unit are recorded. Finally, the measurements of the site tests and the corresponding simulation results are plotted on the same graph in time domain. Fig. 4-1 and Fig. 4-2 compare the model output and site measurements for active power output and wicket gate position. The model is concluded to be satisfactorily 
representing the system as a result of the correlation between the simulation output and site measurements.

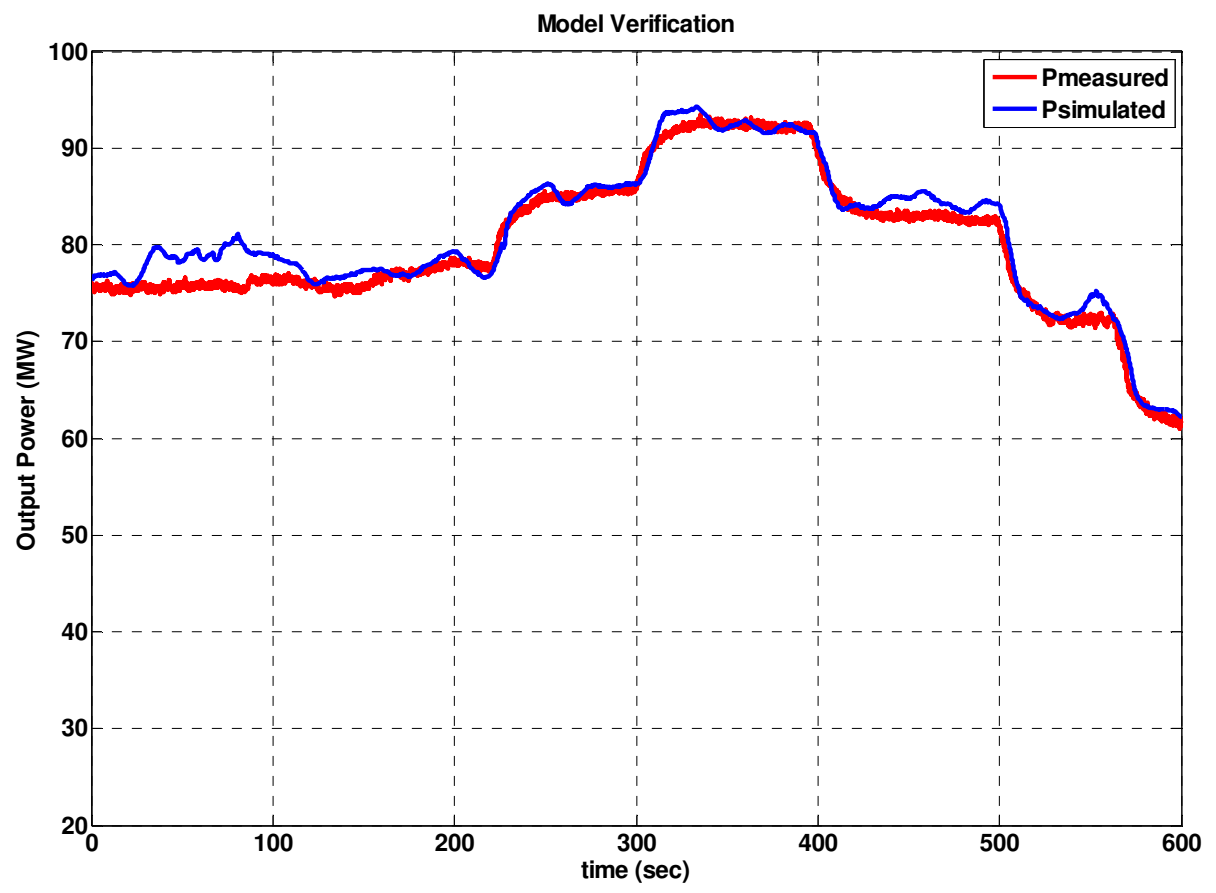

Fig. 4-1: The Graph Comparing the Site Measurements (Red) and Model Output (Blue) for Active Power 


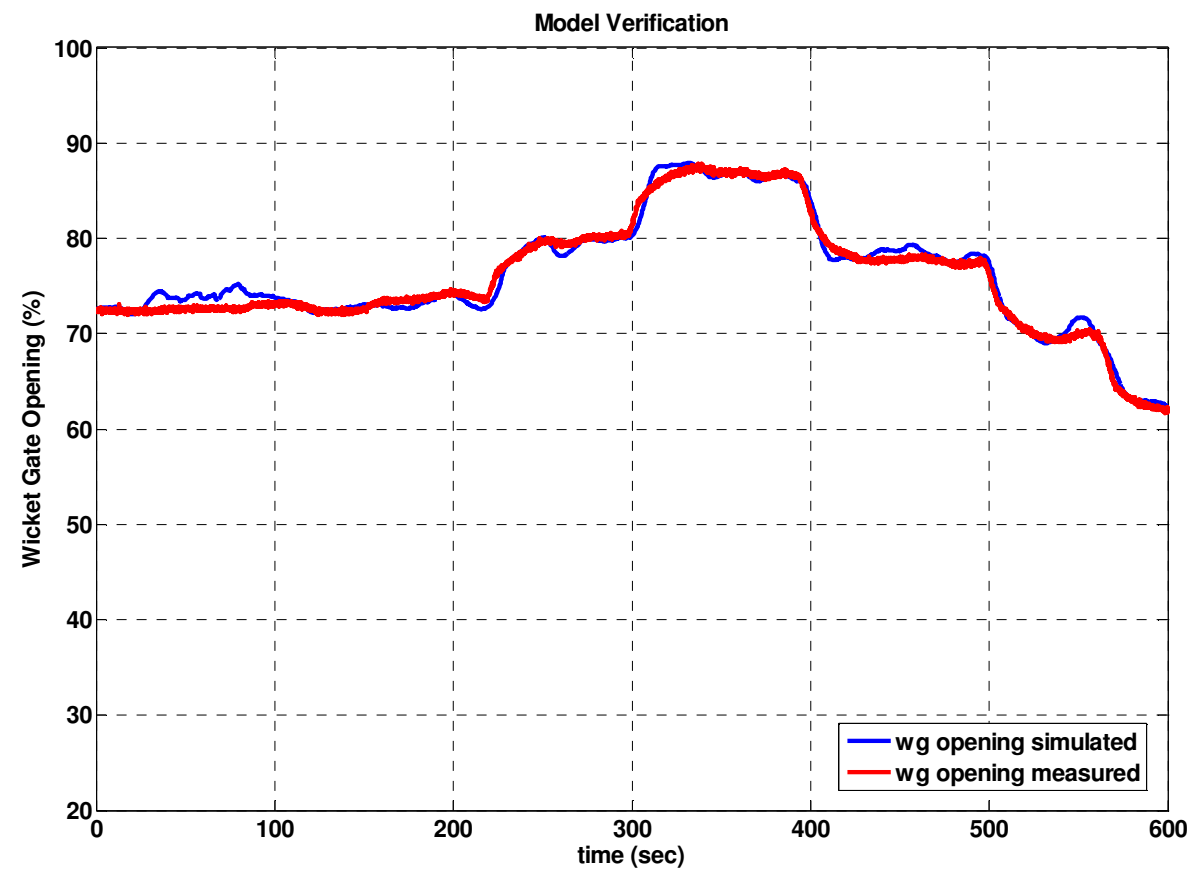

Fig. 4-2: The Graph Comparing the Site Measurements (Red) and Model Output (Blue) for Guide Vane Position

\subsubsection{Evaluation of the Existing Governor Settings}

In order to evaluate the primary frequency control performance of the hydroelectric units with respect to the previously explained expectations of TEIAS regarding the synchronous interconnected operation, site tests have been performed. It is expected to perform the following site tests:

(i) Island Mode Stability Test: The frequency measurement input of the governor is replaced by a fictitious signal. The fictitious signal is generated by specific software which takes the output power of the unit as the input and outputs the simulated frequency of the islanded grid after a change in demand. Note that the island mode simulation is realized by assuming that the only rotating element in the grid is the generator and all the loads are pure impedances. 
(ii) Step Response Tests: The frequency measurement input of the governor is replaced by a fictitious signal. The fictitious signal is a step signal with an offset which simulates a step grid frequency deviation of $200 \mathrm{mHz}$. The output power of the unit and the wicket gate opening signals are recorded in the site tests. The principal aim of these tests is to observe the expected behavior of the unit in case of serious generation outages causing deviations in the grid frequency.

(iii) Frequency Response Tests: The frequency measurement input of the governor is replaced by a fictitious signal. The fictitious signal is a sine signal with an offset which simulates sinusoidal grid frequency deviations of $100 \mathrm{mHz}$. The output power of the unit and the wicket gate opening signals are recorded in the site tests. The principal aim of these tests is to observe the expected behavior of the unit in case of sustained low frequency swings at specific oscillation frequencies.

Since the defacto mode of operation of the hydroelectric power plants when they are interconnected to the grid is the power control mode with frequency influence, this operational mode has been focused on in the simulation studies. The block diagram representation of this control mode is as illustrated in Fig. 3-1. It is important to note here that the acceleration path is an additional rate feedback block which introduces phase lead for the controller for high frequency components of the error signal.

It is important to note here that only the step response tests could be realized on site for this power plant due to compatibility problem of the test equipment and the corresponding software with the measurement and control equipment of the power plant. However, such tests are simulated with the parameters obtained from the speed governor, relying on the results of the model verification study and satisfactory results are obtained. The existing parameter set corresponding to the operational mode of the governor is illustrated in Table 4-1. 
Table 4-1: The Existing Parameter Set

\begin{tabular}{|c|c|c|c|c|c|c|}
\hline Description & $\begin{array}{c}\text { Permanent } \\
\text { Droop }\end{array}$ & $\begin{array}{c}\text { Transient } \\
\text { Droop }\end{array}$ & $\begin{array}{c}\text { Integration } \\
\text { Time Const. } \\
(\mathrm{sec})\end{array}$ & $\begin{array}{c}\text { Proportional } \\
\text { Gain }\end{array}$ & $\begin{array}{c}\text { Accelerating Path } \\
\text { Time Const. (sec) }\end{array}$ & $\begin{array}{c}\text { Accelerating Path } \\
\text { Gain }\end{array}$ \\
\hline Parameter & $\mathrm{K}_{\mathrm{dp}}$ & $\mathrm{K}_{\mathrm{h}}$ & $\mathrm{T}_{\mathrm{h}}$ & $\mathrm{K}_{\mathrm{p}}$ & $\mathrm{T}_{\mathrm{D}}$ for $\mathrm{K}_{\mathrm{D}}$ & $\mathrm{K}_{\mathrm{D}}$ \\
\hline Value & $6 \%$ & $300 \%$ & 1 & 0.1 & 0.2 & 1 \\
\hline
\end{tabular}

It is important to note here that all practical speed governor systems utilize dead band blocks for the frequency deviation signal. However, extensive usage of such blocks both significantly deteriorates the frequency control performance of the units and the frequency stability of the grid. Therefore, for the units with significant capacity, such nonlinear blocks should only be used under specific circumstances with the permission and awareness of the TSO.

As per the expectations of the TSO, the simulation studies are conducted for the following cases:

(i) Island Mode Simulations

(ii) Step Response Simulations

(iii) Frequency Response Simulations

- Island Mode Simulations (+/- 5\% Load Change)

As stated before, in the island mode simulations, it is assumed that the unit feeds an isolated region from the grid and the only rotating element in the islanded network is the unit itself. Hence, it is clear that the rotor speed of the unit determines the frequency of the isolated network. The behavior of the unit is investigated when there is a sudden significant demand change in the isolated network. It is expected that the unit should be capable of maintaining the frequency stability of the isolated network for such disturbances. 
In Fig. 4-3, the frequency of the isolated network is illustrated together with the output of the hydroelectric unit. It is clear from Fig. 4-3 and Fig. 4-4 that due to the peculiar initial response of the unit (i.e., the output power of the unit decreases initially as a result of the penstock dynamics, whereas it was expected to increase), the unit is unstable in island mode. The main reason of the initial false response is the fast proportional response of the governor which should be avoided. As stated in [2], a hydroelectric unit should have large transient gain and long resetting time in order to be stable in island mode.
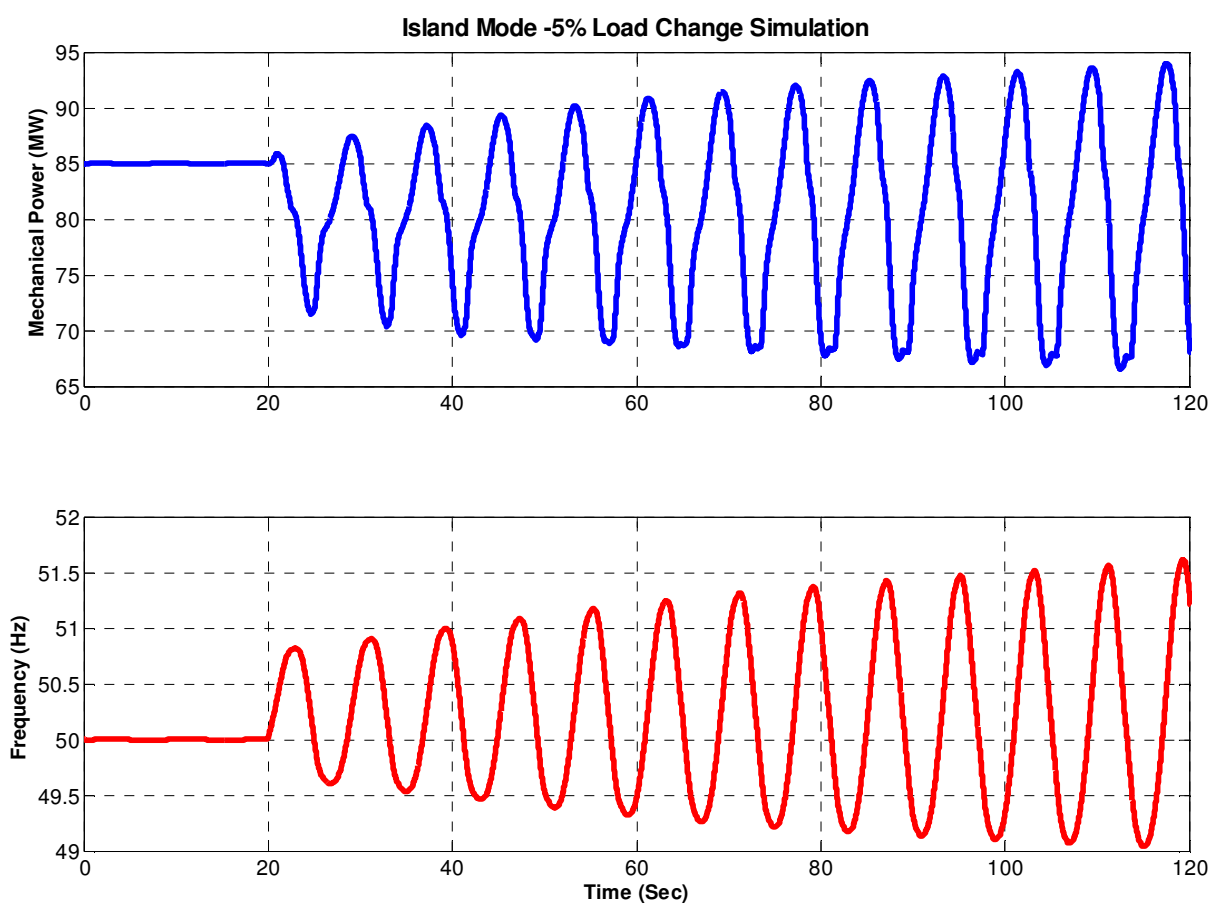

Fig. 4-3: Island Mode Simulation (-5\% Load Change) with the Existing Governor Settings 

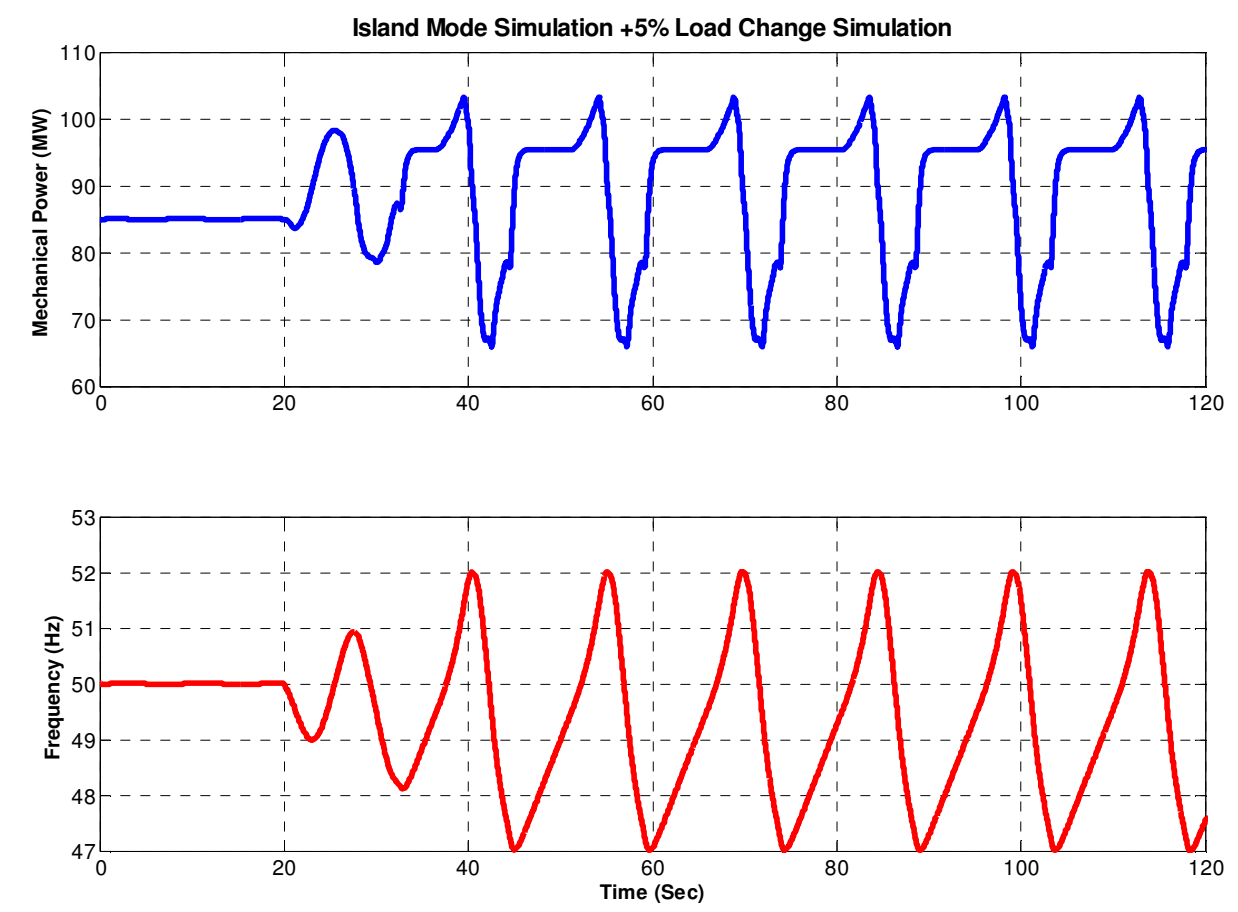

Fig. 4-4: Island Mode Simulation (+5\% Load Change) with the Existing Governor Settings

\section{- $200 \mathrm{mHz}$ Step Frequency Deviation Simulations}

As per the instructions of the UCTE ground rules [4], each control area should provide its share of the total operational reserve (which is defined as $3000 \mathrm{MW}$, that corresponds to the outage of the biggest nuclear electricity generation facility in ENTSO-E CESA) in approximately 30 seconds. Since the total share of hydroelectric power plants in overall electricity generation profile of Turkey is about 30\%, the frequency control performance of the Turkish Power System is significantly dependent on the performance of the considerable size hydroelectric power plants. It is clear that the requirements for stability of speed control under island mode of operation conflict with the requirements for the fast response of the hydroelectric unit. 
The simulation results regarding the output power response of the unit against step frequency deviations are illustrated in Fig. 4-5 and in Fig. 4-6. It can be seen from the simulation results that the settling time of the unit is about 15 seconds, which is well below the maximum limit of the activation of the primary frequency control reserve. UCTE ground rules state that, in addition to activating the primary frequency control reserve in 30 seconds, the unit should also be capable of maintaining its response for 15 minutes in case the frequency is not restored. Although this expectation is a serious concern for the thermal units due to the pressure changes as a result of the slow boiler control (note that those plants are operated in the "boiler follows the turbine" mode); for hydroelectric power plants with large reservoirs (i.e., for dam type hydroelectric power plants), this expectation is not a serious concern due to the large potential energy of the stored water in the reservoir. Since Sir HPP also has a considerable reservoir size, all units are capable of maintaining their response for 15 minutes, in case the frequency is not restored or the secondary controller orders them to operate in that fashion.
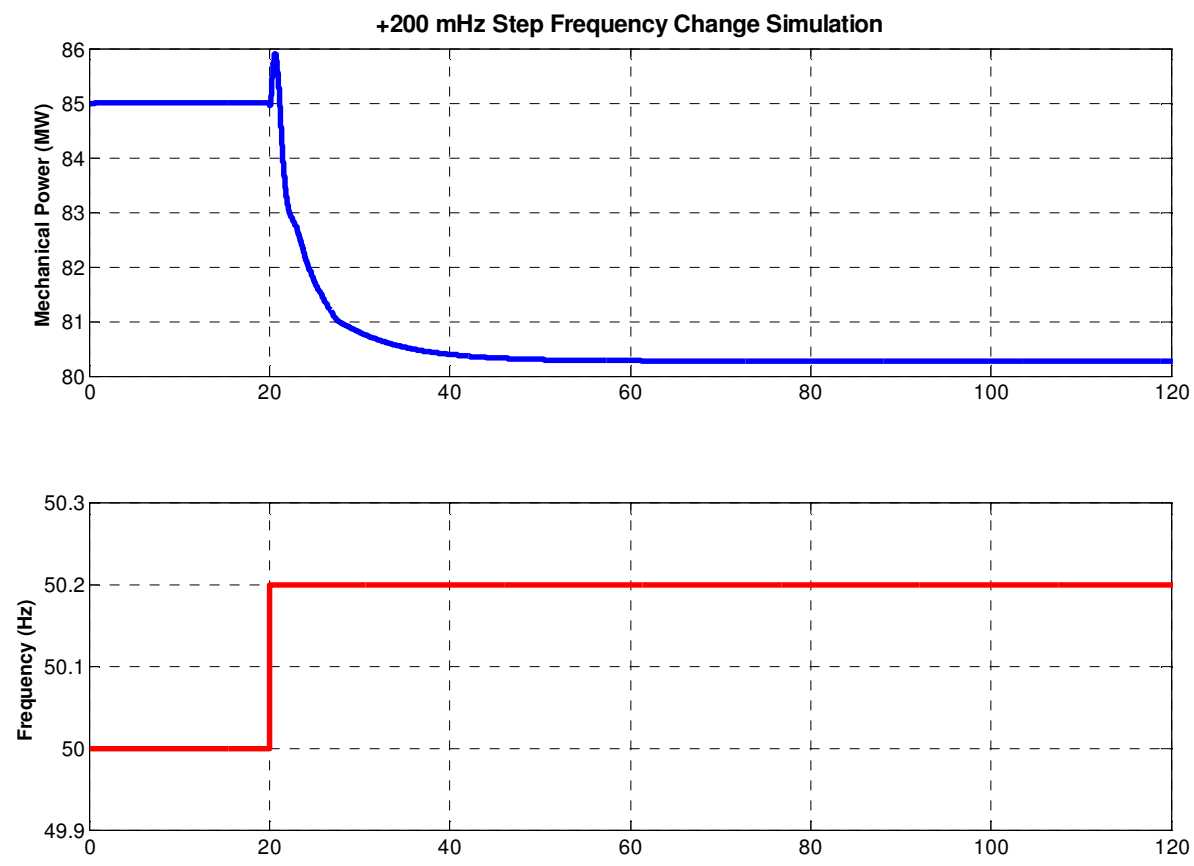

Fig. 4-5: +200mHz Step Frequency Deviation Simulation with the Existing Governor Settings 

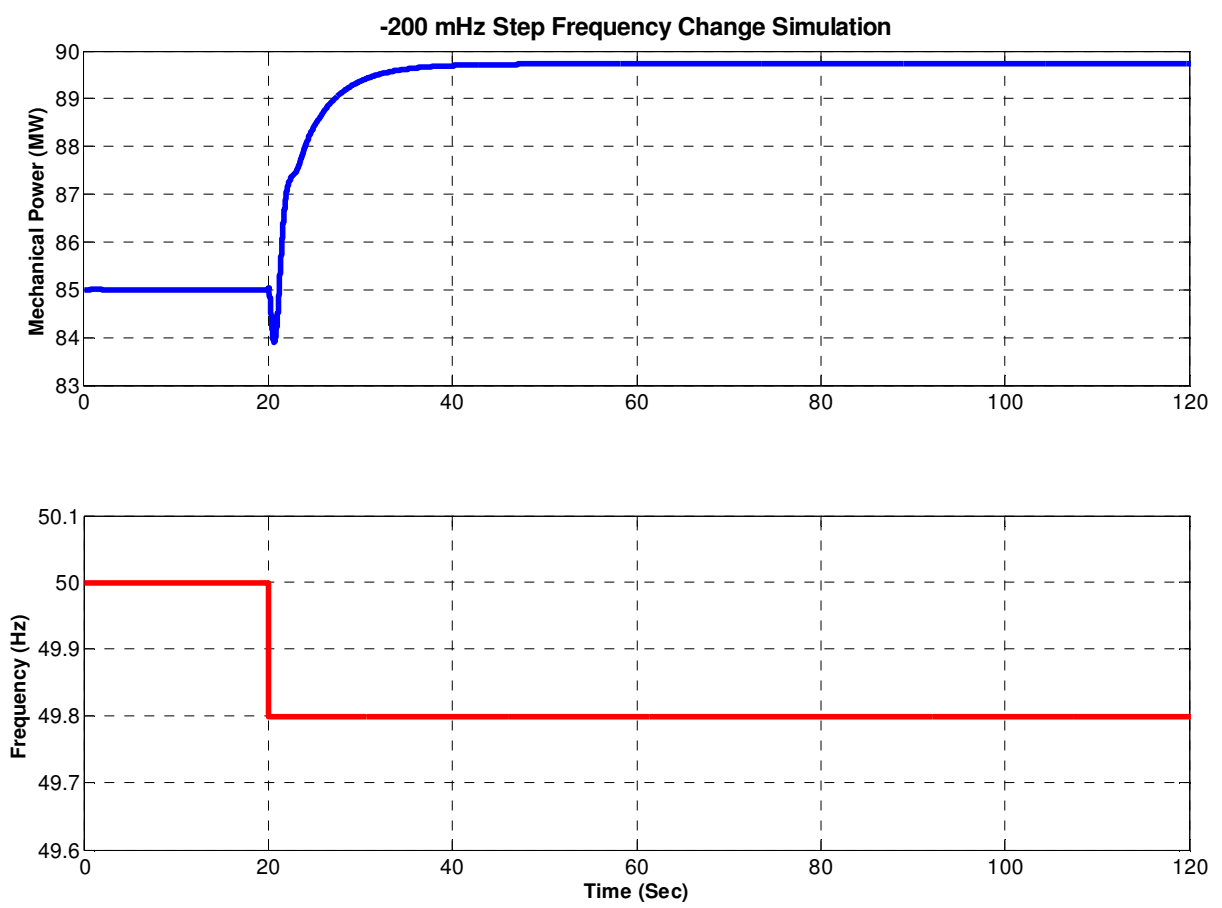

Fig. 4-6: -200mHz Step Frequency Deviation Simulation with the Existing Governor Settings

\section{- Frequency Response Simulations}

In large interconnected power systems like the ENTSO-E CESA network, possible negative effects of single units or power plant on the overall system performance are less observable. In other words, such undesired effects are negligible considering the size of the interconnected system and hence may be tolerated. However, such an approach is not admissible for the Turkish power system due to the considerations listed below.

(i) The installed capacity of the Turkish Power System is about 1/12 of that of the ENTSO-E CESA System

(ii) $1 / 3$ of total eectricity demand is supplied by hydroelectric power plants in the Turkish Power System in the peak hours. 
(iii) The system includes long transmission lines which also mean longitudinal system structure and relatively weak interconnection between east and west (Note that the operation of such long transmission lines can only be managed by series capacitors).

Therefore, prior to the synchronous interconnection of the Turkish Power System to ENTSO-E CESA System, it has to be ensured that each individual generation unit contributes to the stability of the overall system frequency by means of suitable control structures and parameters, also taking into account its specific dynamic characteristics.

In order to observe the effect of the governor settings on system damping in the frequency bandwidth of the possible inter area oscillations after the interconnection of the Turkish Power System with the ENTSO-E CESA system, the frequency response of the turbine governor system is investigated. The simulations are carried out by injecting a pure sinusoidal frequency signal with an offset value instead of the frequency measurement signal. The simulation results are evaluated by investigating the magnitude and phase of the first harmonic of the steady state output power. It is important to note here that although the actual system is nonlinear, the linear analysis tools result well as a result of the principle of harmonic balance as explained in the Section 3.2.2. The Bode Plot of the system (from output power to speed measurement) is illustrated in Fig. 4-7.

The ideal mechanical response from a generating unit against sinusoidal frequency oscillations is to provide a torque component that is 180 degrees out of phase with the frequency deviations, regarding the sytem damping for low frequency inter area oscillations.

Investigating Fig. 4-7, it can clearly be observed that once the frequency of the oscillations increases, the phase lag of the output power increases, which produces a torque component that is opposite to what is being desired. The simulation results of the system against $100 \mathrm{mHz}$ amplitude $0.15 \mathrm{~Hz}$ sinusoidal frequency oscillations is 
illustrated in time domain in Fig. 4-8. The phasor corresponding to the mechanical power of the machine is plotted in phasor domain in Fig. 4-9 where the $\mathrm{x}$ axis (i.e., the reference) is in phase with the damping torque component of the unit (i.e., in phase with $-\Delta \mathrm{w})$.
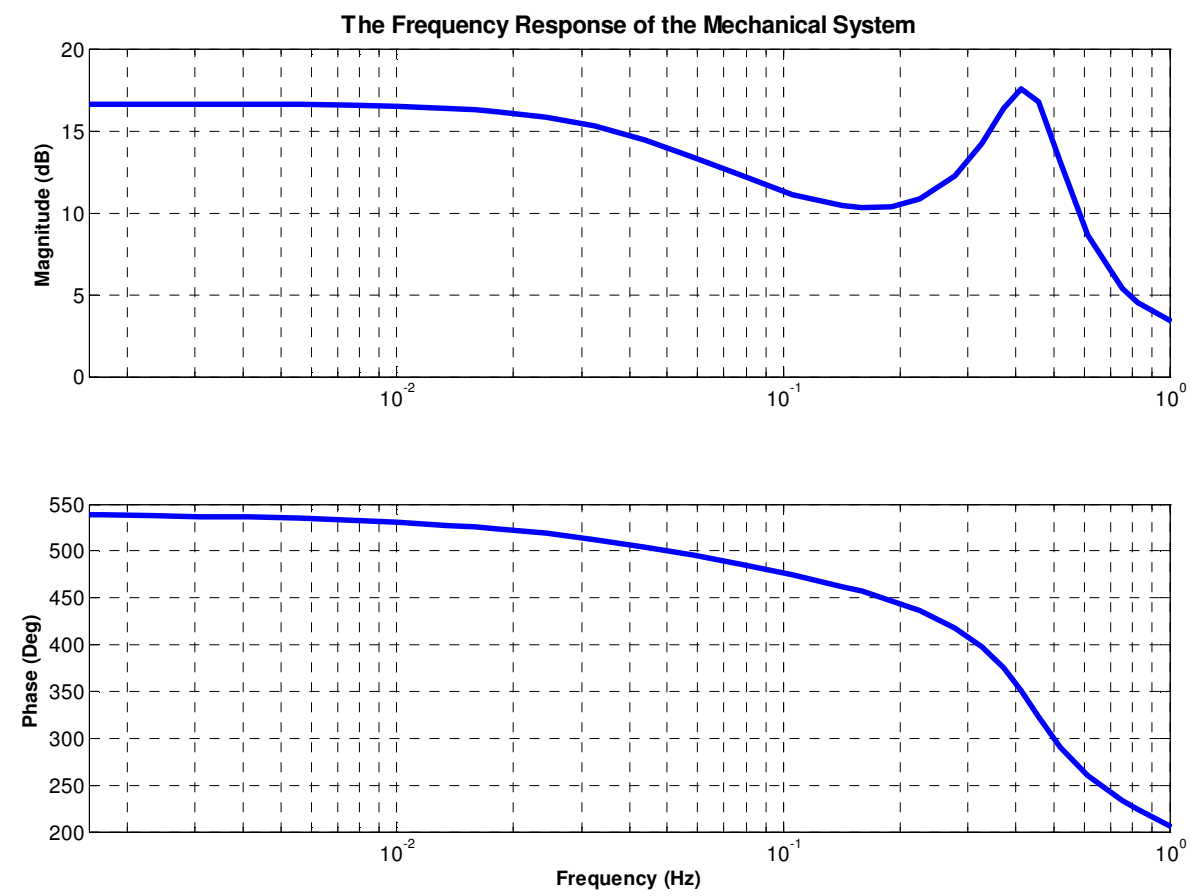

Fig. 4-7: The Frequency Response of the System with the Existing Governor Settings 

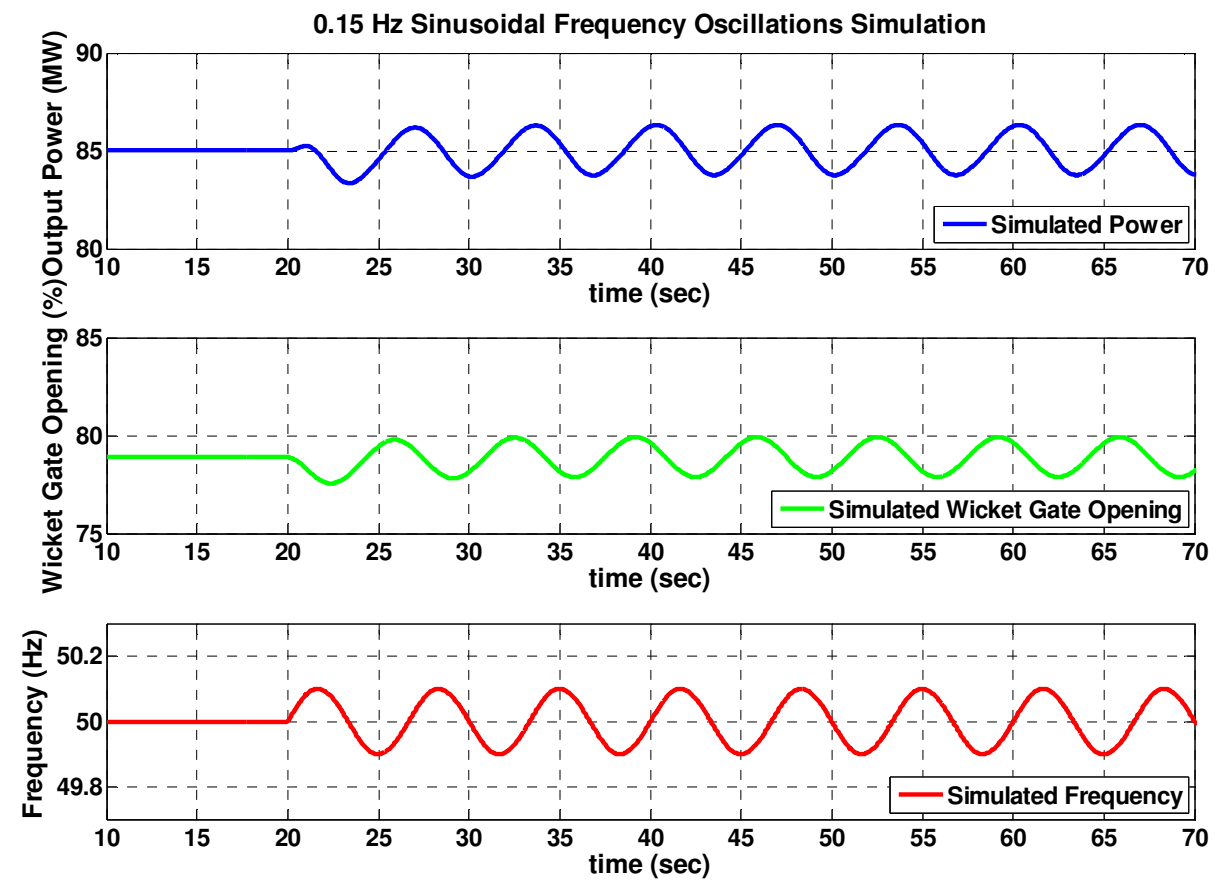

Fig. 4-8: 0.15 Hz Sinusoidal Frequency Oscillations Simulation with the Existing Governor Settings

As can directly be assessed by investigating the phase of the mechanical power response from Fig. 4-9, the turbine governor of the unit produces a torque component that constitutes a source of negative damping, hence threatening both the frequency stability of the system and the steady state stability of the system. 


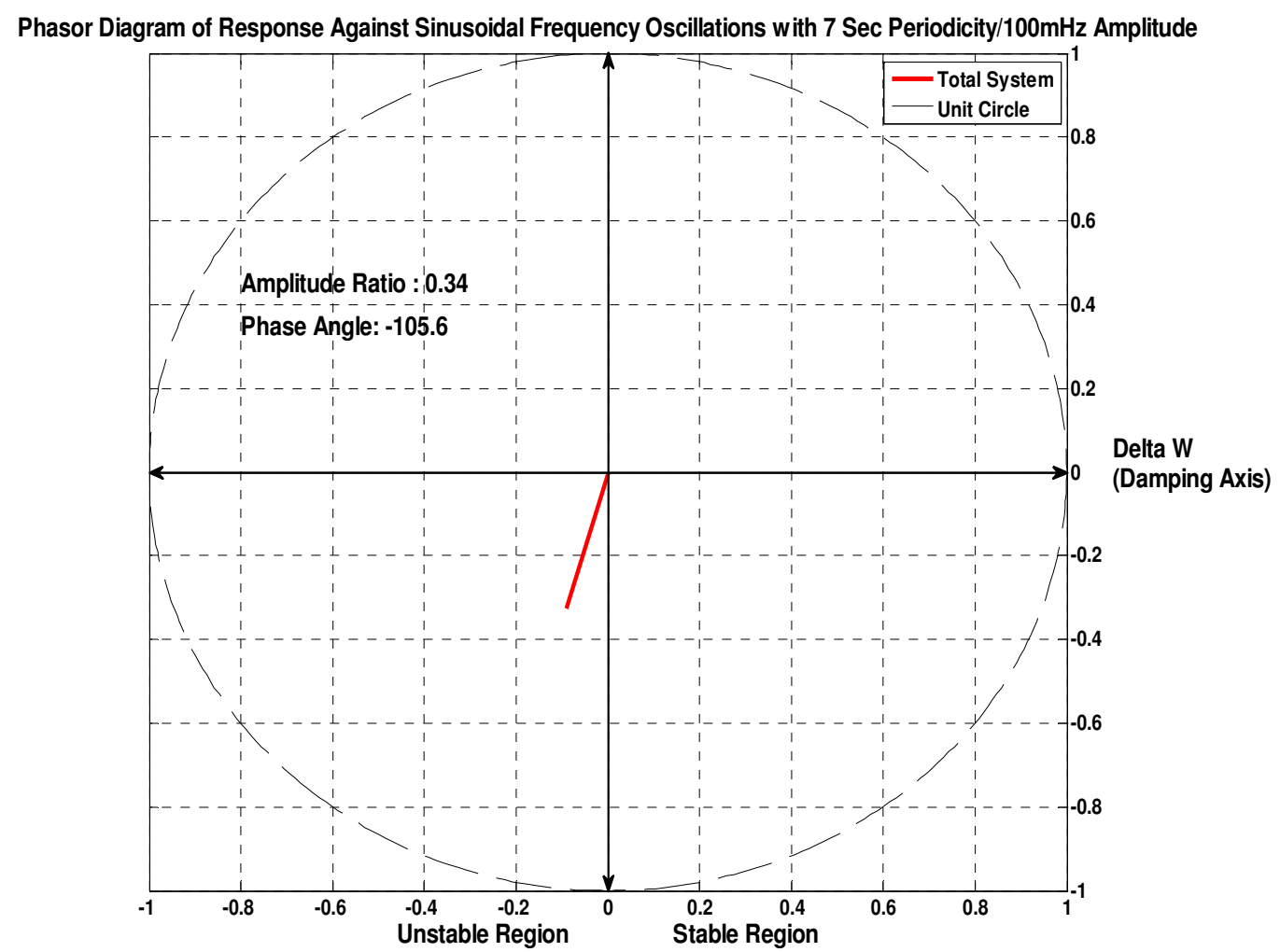

Fig. 4- 9: The Phasor Diagram of the Mechanical Power Response of the Unit against Sinusoidal Frequency Input with the Existing Governor Settings

Evaluating the primary frequency control performance of the sample unit, with the existing governor settings, from this point of view, it is observed that,

(i) The existing governor settings of the hydroelectric power plant are unstable in island mode. However there is automatic parameter changeover in case of an island detection during network operation.

(ii) The settling time of the mechanical power response against step frequency deviations for the hydroelectric unit was about 15 seconds.

(iii) The mechanical response of the unit would deteriorate the system damping in case of possible sustained (or growing) low frequency inter area oscillations $(\sim 0.15 \mathrm{~Hz})$ as explained in the part 2.5.2 (i.e., in Fig. 229). 


\subsubsection{The Proposed Governor Settings}

As per the expectations of the TSO, the simulation studies regarding the retuning of the governor are conducted with the following constraints:

(i) The unit should be stable in island mode as stated in [5].

(ii) The settling time of the unit against step frequency deviations should be comparable with 30 seconds (If possible together with (iii)).

(iii) The turbine governor of the unit should not deteriorate the system damping performance against inter area power swings (If this is not possible due to physical constraints, the smallest possible adverse effect should be ensured).

The tuning studies are carried out with the above explained constraints as will be explained in the proceeding parts. The proposed parameter set, corresponding to the operational mode of the governor and obtained from the interpretation of the simulation results, is tabulated in Table 4-2.

Table 4-2: The Proposed Parameter Set (1st Parameter Set)

\begin{tabular}{|c|c|c|c|c|c|c|}
\hline Description & $\begin{array}{c}\text { Permanent } \\
\text { Droop }\end{array}$ & $\begin{array}{c}\text { Transient } \\
\text { Droop }\end{array}$ & $\begin{array}{c}\text { Integration } \\
\text { Time Const. } \\
(\mathrm{sec})\end{array}$ & $\begin{array}{c}\text { Proportional } \\
\text { Gain }\end{array}$ & $\begin{array}{c}\text { Accelerating Path } \\
\text { Time Constant (sec) }\end{array}$ & $\begin{array}{c}\text { Accelerating Path } \\
\text { Gain }\end{array}$ \\
\hline Parameter & $\mathrm{K}_{\mathrm{dp}}$ & $\mathrm{K}_{\mathrm{h}}$ & $\mathrm{T}_{\mathrm{h}}$ & $\mathrm{K}_{\mathrm{p}}$ & $\mathrm{T}_{\mathrm{D}}$ for $\mathrm{K}_{\mathrm{D}}$ & $\mathrm{K}_{\mathrm{D}}$ \\
\hline Value & $\% 6$ & $\% 600$ & 3 & 0.1 & 0.2 & 1 \\
\hline
\end{tabular}

During the tuning study, since the basic motivation is to remove the component of the mechanical torque that deteriorates the system damping performance, the phase characteristics of the transfer function of the governor and turbine is focused on in the first step. Provision of phase lead from speed input to mechanical power of the unit helps the mechanical parts of the unit not to amplify the frequency oscillations. However, under certain circumstances such as the hydroelectric units with very long 
water starting times, the desired phase lead is impossible to be achieved. In such cases, the optimum parameter set turns out to be the one where the magnitude of the undesired torque component is minimized.

Secondly, considering the tradeoff between the fast transient response and system stability (both concerning the frequency stability of the Turkish Power System [5] and the damping performance of the interconnected system against possible inter area oscillations), the best settling time is achieved using a parameter set among the candidate parameter sets which do not cause any torque components deteriorating the damping torque of the unit.

Finally, the selected parameter set is checked for stability in island operation. In case this criterion is not satisfied, another parameter set satisfying the first criterion is assessed.

\section{- Frequency Response Simulations}

As stated before, a nonlinear hydroelectric turbine model is utilized in this study. However, since the effect of the dynamical system in a certain frequency range is investigated, analysis of the first harmonic of the mechanical power response provides sufficient information about the behavior of the dynamic system as it is the case for linear systems.

In order to have a rough idea about the effect of a single parameter in the phase characteristics of the system, a simplified linear turbine model as stated in [2] is utilized together with the governor model. Writing down the explicit transfer function for the simplified linear governor model, it can directly be seen that the desired phase lead can simply be achieved by increasing the time constant and the gain of the transient gain reduction feedback loop of the PD governor.

Afterwards, the effectiveness of the initial tuning study is checked through the simulations made by the nonlinear turbine-governor model as mentioned in sections 3.3.2.1. and 3.3.2.2. The frequency response of the first harmonic of the mechanical 
system is as plotted in Fig. 4-10. From Fig. 4-10, it can easily be seen that with the addition of phase lead (i.e., phase lag for mechanical power), the mechanical response of the turbine governor system will not deteriorate the natural damping of the machine for the frequency swings due to poorly damped low frequency inter area modes (around $0.15 \mathrm{~Hz}$ ). However, the tradeoff of this parameter set is the increased settling time due to the phase lag caused in the frequency range of load frequency control (i.e., $0.01 \mathrm{~Hz}$ to $0.1 \mathrm{~Hz}$ ), which means that the turbine governor will respond in a slower manner against frequency deviations those occur as a result of the generation-load imbalance, a matter of fact due to the demand variations or outages.
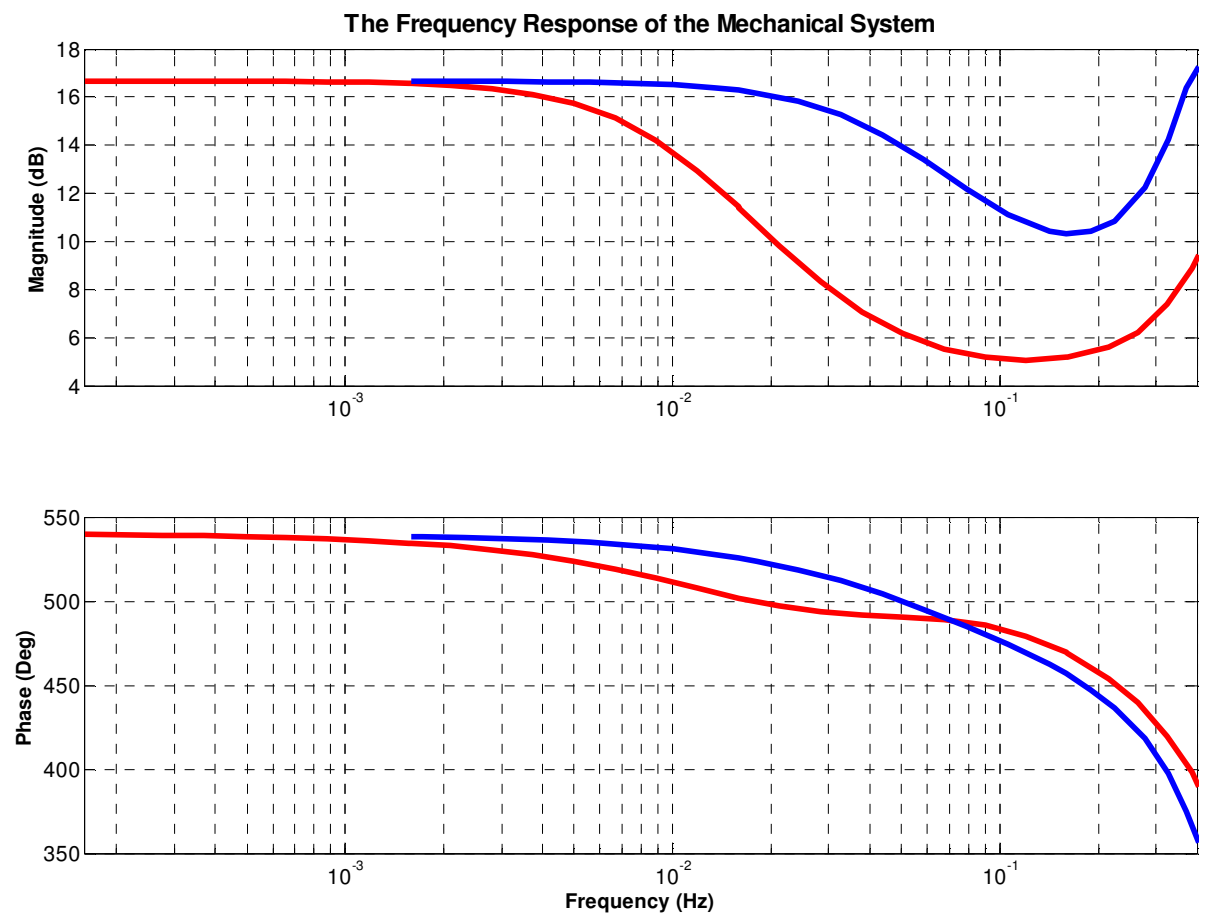

Fig. 4- 10: The Frequency Response Plots of the Unit with the Existing Governor Settings (Blue) and the Proposed Governor Settings (Red)

The simulation results of the system against $100 \mathrm{mHz}$ amplitude $0.15 \mathrm{~Hz}$ sinusoidal frequency oscillations is illustrated in time domain in Fig. 4-11. The phasor corresponding to the mechanical power of the machine is plotted in phasor domain in 
Fig. 4-12. As can directly be assessed by comparing Fig. 4-12 with Fig. 4-9, the turbine governor of the unit produces a torque component that constitutes a source of slightly positive damping, hence neither enhancing the damping performance of the system nor threatening the frequency stability and the steady state stability of the system.
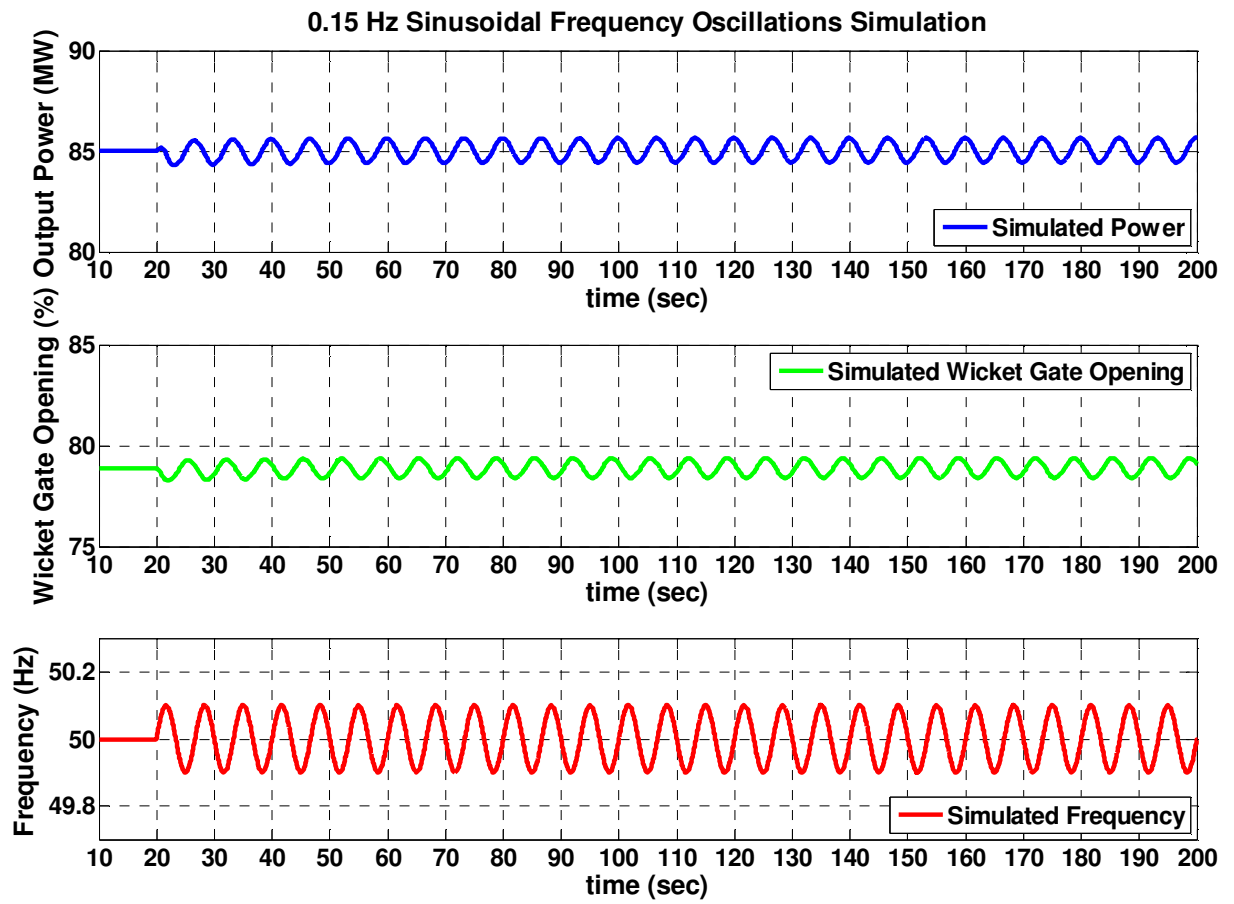

Fig. 4-11: 7 Sec Sinusoidal Frequency Oscillations Simulation with the Proposed Governor Settings 


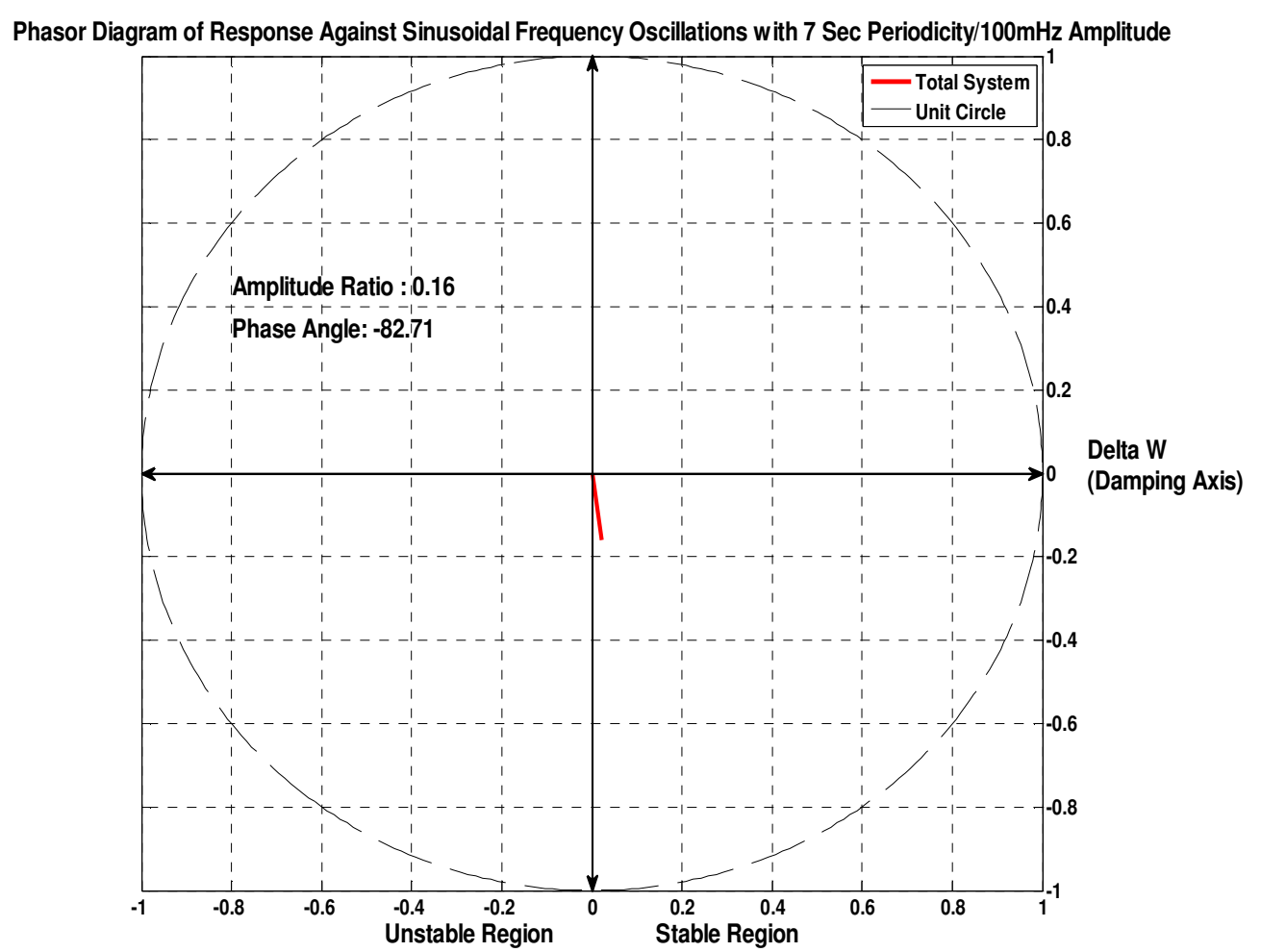

Fig. 4-12: The Phasor Diagram of the Mechanical Power Response of the Unit against Sinusoidal Frequency Input with the Proposed Governor Settings

- Island Mode Simulations (+/- 5\% Load Change)

After the required phase lead for the speed (i.e., lag for mechanical power) around $\sim 0.15 \mathrm{~Hz}$ is satisfied by trying several parameter sets, it should be checked that the proposed parameter set satisfies the island mode stability criterion as discussed in [5]. This simply stems from the fact that due to the significant share of hydroelectric power plants in the overall electricity generation profile, the island mode stability criterion of the considerable size hydroelectric units turn out to be a keystone for the "frequency stability" of the Turkish Power System as discussed in [5] and [7], regardless of the interconnection studies.

The island mode stability simulations are carried out in a similar manner to the ones 
in 4.2.1.2. and the simulation results are illustrated in Fig. 4-13 and Fig. 4-14. from the simulations, it is concluded that the unit is stable in island mode of operation.
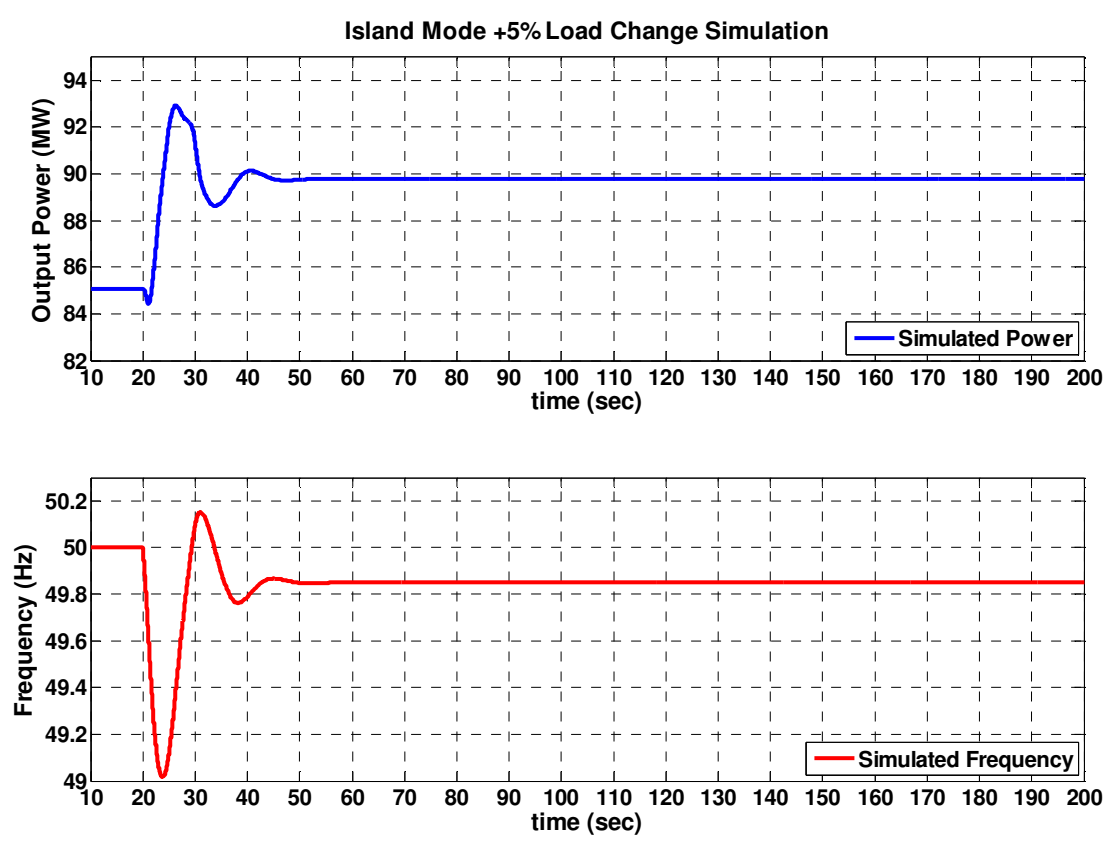

Fig. 4-13: Island Mode Simulation (+5\% Load Change) with the Proposed Governor Settings 

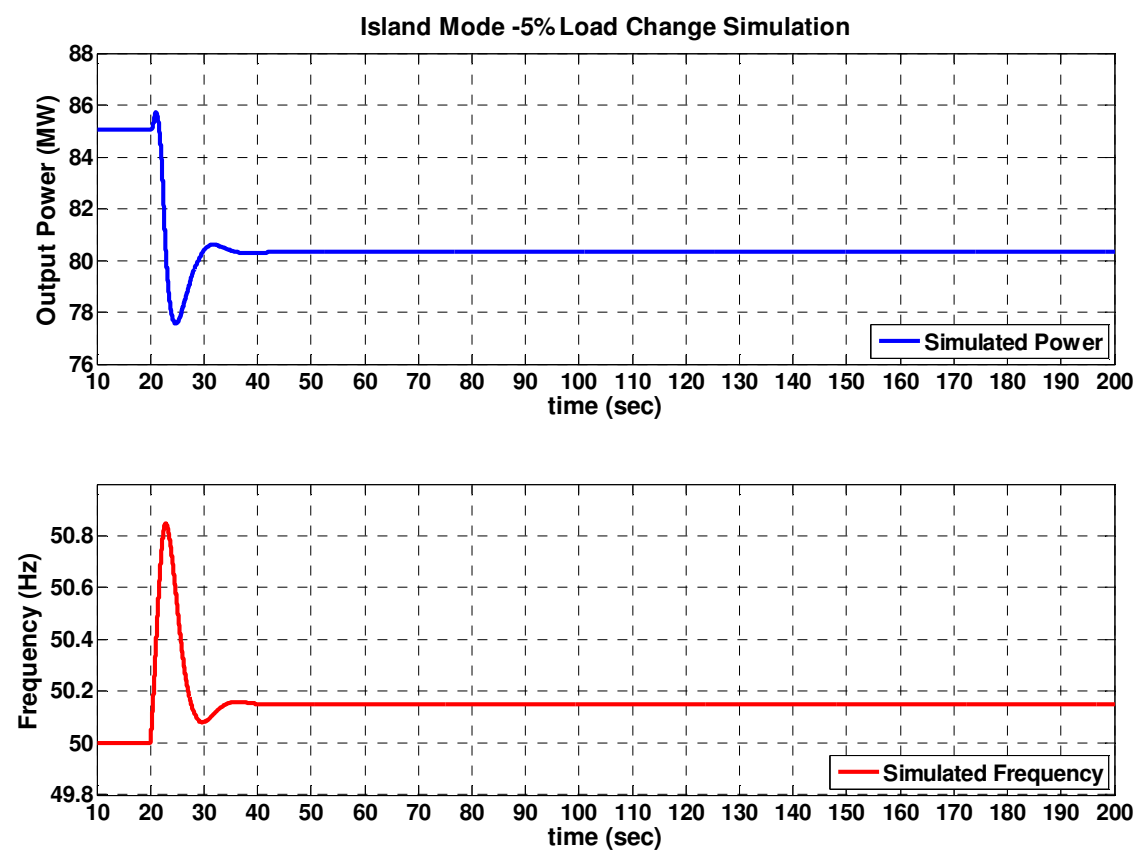

Fig. 4-14: Island Mode Simulation (-5\% Load Change) with the Proposed Governor Settings

\section{- $200 \mathrm{mHz}$ Step Frequency Deviation Simulations}

It is important to note here that the only consideration for the increased transient droop is the settling time expectation of the TSO, since with increased transient droop and time constant of the transient gain reduction feedback loop of the PD governor, the settling time of the mechanical response of the unit against fast frequency deviations significantly increases, which is an undesired behavior for the primary frequency control performance of the unit (hence the contingency handling capability of the system, i.e., the frequency stability). The UCTE ground rules determine the settling time of the control area against step frequency deviations as 30 seconds [2]. Therefore, it is expected that the settling time of the individual units against step frequency deviations should be comparable with 30 seconds.

In most cases, it is impossible to satisfy the expectations regarding the damping performance of the system and settling time, especially for large size hydroelectric 
units. In such a dilemma, for large size hydroelectric units, since system dynamic stability is inevitable, the tradeoff of the governor settings which do not deteriorate the system damping is the increased settling time, that may cause unintended power transfer to the ENTSO-E CESA System in case of severe incidences, since the frequency will not be restored in a short time interval causing the unit to continue responding. It is important to note here that this phenomenon will be compensated by the secondary control action and is not of primary concern as long as the total amount of primary frequency control reserve allocated to the hydroelectric power plants does not exceed a certain range throughout the system.

In Fig. 4-15, Fig. 4-16, the corresponding simulation results with the proposed governor parameters are plotted.
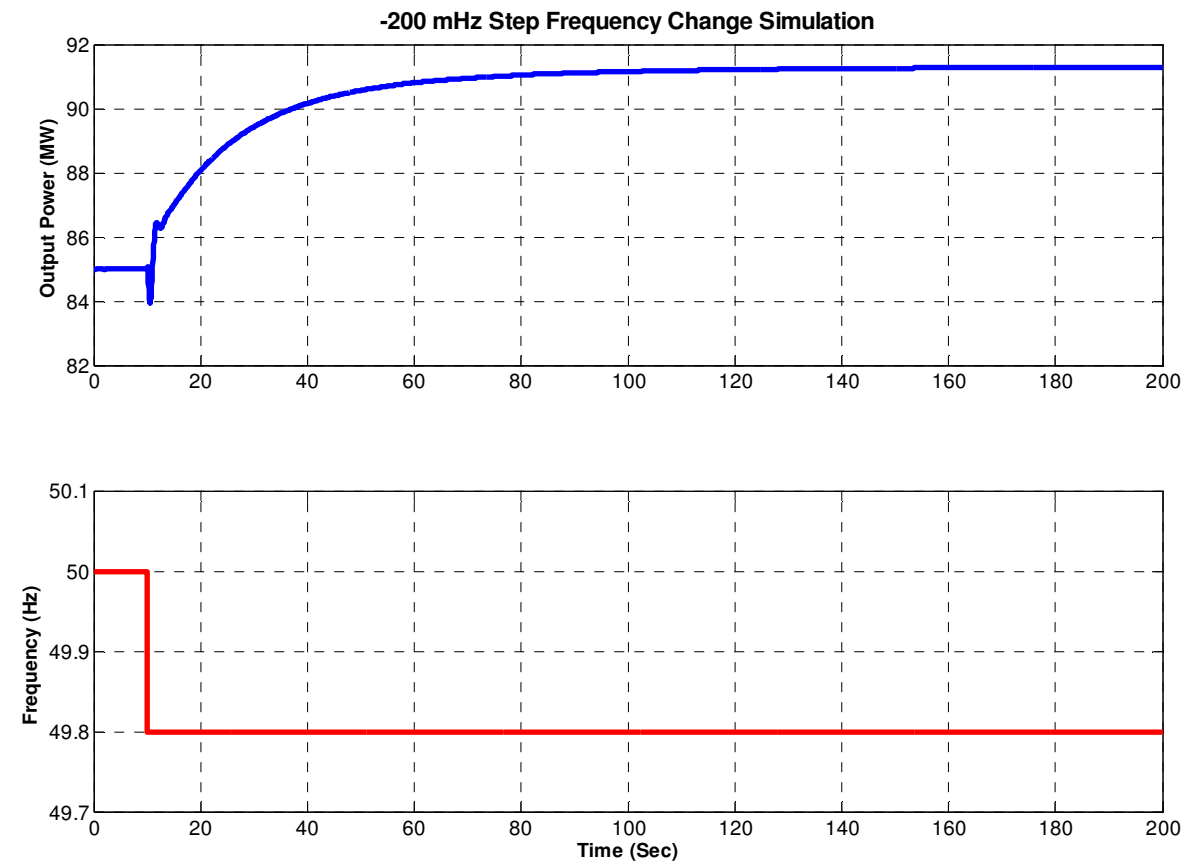

Fig. 4-15: The Response of Mechanical Power against Step Frequency Deviations with $6 \%$ Permanent Droop 

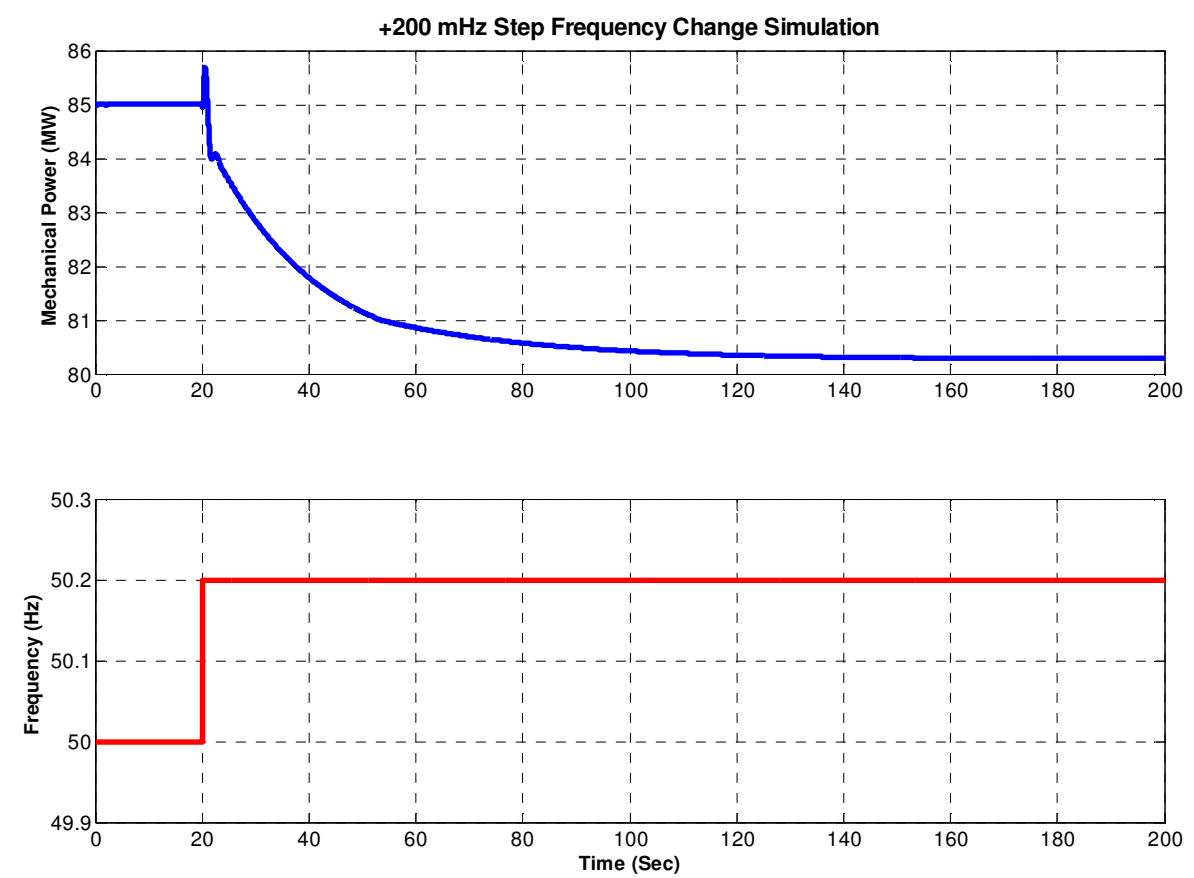

Fig. 4-16: The Response of Mechanical Power against Step Frequency Deviations with $6 \%$ Permanent Droop

Another method to overcome this undesired effect is to reduce the amount of response of the units to frequency deviations by increasing the value of the "Permanent Droop" of the units. By this system wide approach, both the settling time of the unit gets closer to 30 seconds with the parameter sets satisfying the stability criterions and the total amount of primary frequency control reserve allocated to hydroelectric power plants decreases which does not cause violation of the (n-1) rule for Turkish Power System in interconnected operation with ENTSO-E CESA System, due to the decreased reserve requirement of the system as a benefit of the interconnected operation. In Fig. 4-17, the corresponding simulation results with the proposed governor parameters are plotted. 

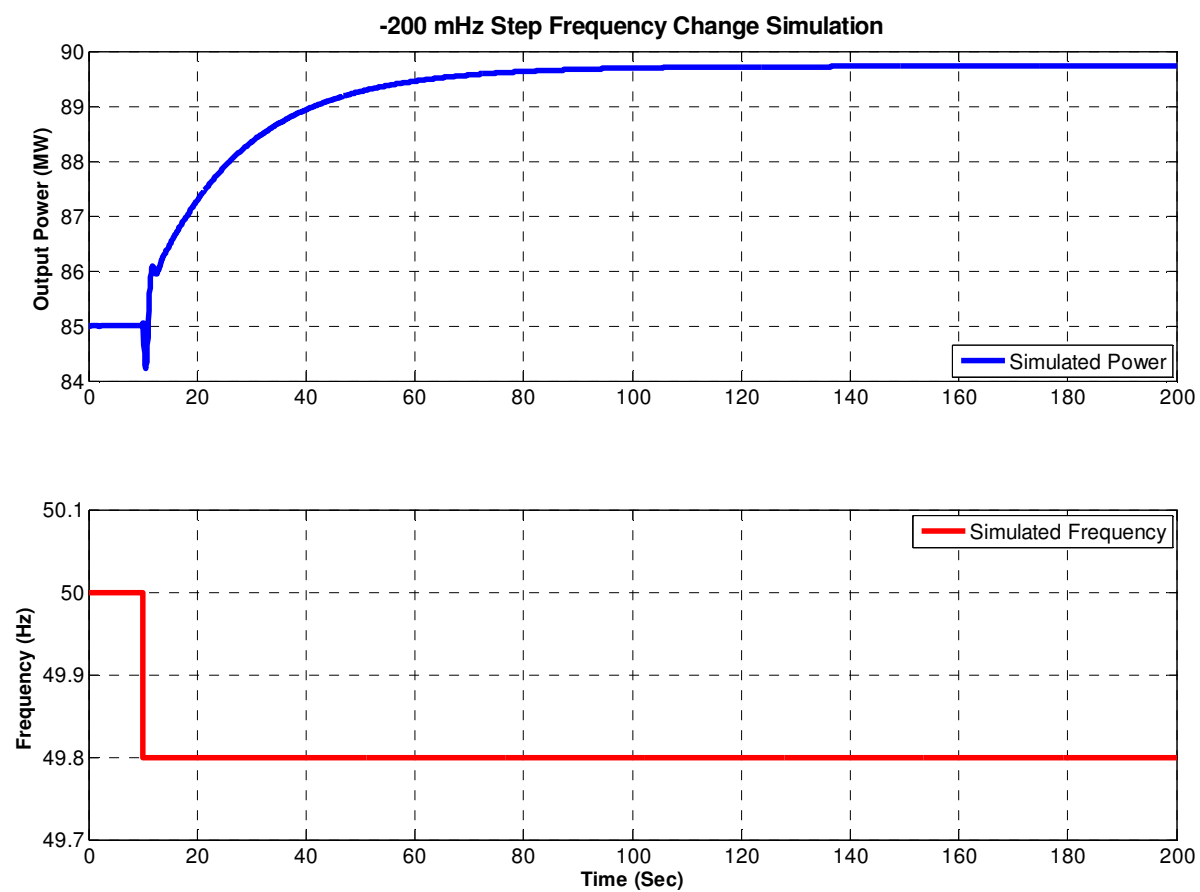

Fig. 4-17: The Response of Mechanical Power against Step Frequency Deviations with $8 \%$ Permanent Droop

Therefore, another suitable parameter set can be stated as tabulated in Table 4-3, by considering both the settling time, the frequency stability and the damping performance of the system.

Table 4-3: The Proposed Parameter Set (2nd Parameter Set)

\begin{tabular}{|c|c|c|c|c|c|c|}
\hline Description & $\begin{array}{c}\text { Permanent } \\
\text { Droop }\end{array}$ & $\begin{array}{c}\text { Transient } \\
\text { Droop }\end{array}$ & $\begin{array}{c}\text { Integration } \\
\text { Time Const. }\end{array}$ & $\begin{array}{c}\text { Proportional } \\
\text { Gain }\end{array}$ & $\begin{array}{c}\text { Accelerating Path } \\
\text { Time Constant }\end{array}$ & $\begin{array}{c}\text { Accelerating Path } \\
\text { Gain }\end{array}$ \\
\hline Parameter & $\mathrm{K}_{\mathrm{dp}}$ & $\mathrm{K}_{\mathrm{h}}$ & $\mathrm{T}_{\mathrm{h}}$ & $\mathrm{K}_{\mathrm{p}}$ & $\mathrm{T}_{\mathrm{D}}$ for $\mathrm{K}_{\mathrm{D}}$ & $\mathrm{K}_{\mathrm{D}}$ \\
\hline Value & $\% 8$ & $\% 600$ & 3 & 0.1 & 0.2 & 1 \\
\hline
\end{tabular}




\subsubsection{Assessment of the Effectiveness of the Proposed Governor Settings in Multi Machine Network}

The tuning study of the governor of the hydroelectric unit is performed in a single machine infinite busbar model. However, in the actual power system, the hydroelectric unit is operated together with other types of generation facilities. Therefore, the frequency stability of the power system is strongly dependent on the combination of the effect of different dynamical characteristics of different technologies. This fact makes multi machine simulations an integral part of the study before concluding about the effectiveness of the proposed governor settings. In addition, while performing the governor tuning studies, the generator and exciter of the unit are not modeled, which are the initiators of the possible poorly damped inter area oscillations. This very low frequency electromechanical phenomenon turns into a generation-load imbalance problem in case of sustained (or very poorly damped) inter area swings, as it is the case in this study.

Therefore, in order to demonstrate the effect of the retuned parameter set of the hydroelectric unit to the damping performance of the system against low frequency $(\sim 0.15 \mathrm{~Hz})$ inter area oscillations, Kundur's Two Area Power System in [14] is utilized. In the mentioned two area four machine power system in [14], two out of three transmission lines between the two areas are put in service. The effectiveness of the proposed governor settings in multi machine systems is analyzed by replacing the model of the machine in [14], in which the observability of the poorly damped modes were found to be the greatest, with that of the hydroelectric power plant of interest. It should be noted here that since per unit system is utilized in the models, this replacement does not cause any ambiguities in the model.

In the simulations, Area 1 has been assumed to represent the ENTSO-E CESA system; therefore, the inertias of the machines in Area 1 have been increased so as to create low frequency inter area oscillations $(\sim 0.15 \mathrm{~Hz})$, whereas Area 2 has been assumed to represent the Turkish Power System and no modification has been made for the machines in Area 2, except the replacement of one of the machines with the 
developed simulation model of interest. The two coherent generators (observed in simulations) both in Area 1 and in Area 2 were assumed to have the same voltage controllers with $\Delta \mathrm{w}$ type PSSs.

The steady state stability of a power system is strongly dependent on the operating conditions (i.e., the operating point, the equilibrium point) as explained in Chapter 2 and Chapter 3. Therefore, the coherent machines in the two control areas are set to equal power and voltage output in order to focus only on the effect of the mechanical parts of the unit on the damping performance of the system, but not the other factors effecting inter area oscillations such as loading of the machines, output voltage and controller structure of the unit.

Within this approach, 50\% of the electricity generation in Area 2 (i.e., the representative Turkish Power System) is scheduled to hydroelectric power plants, which is a realistic worst case dispatch scenario, considering the $30 \%$ share of hydroelectric power plants in total installed capacity of Turkish Power System. Such a scenario may occur due to market conditions in Turkey in spring season, when the hydroelectric power plants are operated close to their full capacity and the total demand is at minimum. Further, Area 1 is assumed to transfer 400 MW to Area 2 in order to create a weak system configuration from the viewpoint of small signal rotor angle stability, in addition to simulating a permanent fault causing outage in one of the two transmission lines between the two areas. This contingency ends up with a weaker transmission topology assuming that the scheduled transaction between the two areas is kept constant after the incident.

It has been observed from the simulations that the generators both in Area 1 and in Area 2 oscillate coherently and hence represent the expected mode shapes of the Turkish Power System [16] and ENTSO-E CESA System after interconnection. Fig. 4-18 illustrates the frequency of the two power systems after the outage of one of the transmission lines with the existing governor settings of the hydroelectric unit of interest, whereas Fig. 4-19 illustrates the frequency of the two power systems after 
the outage of one of the transmission lines with the proposed governor settings of the hydroelectric unit of interest.

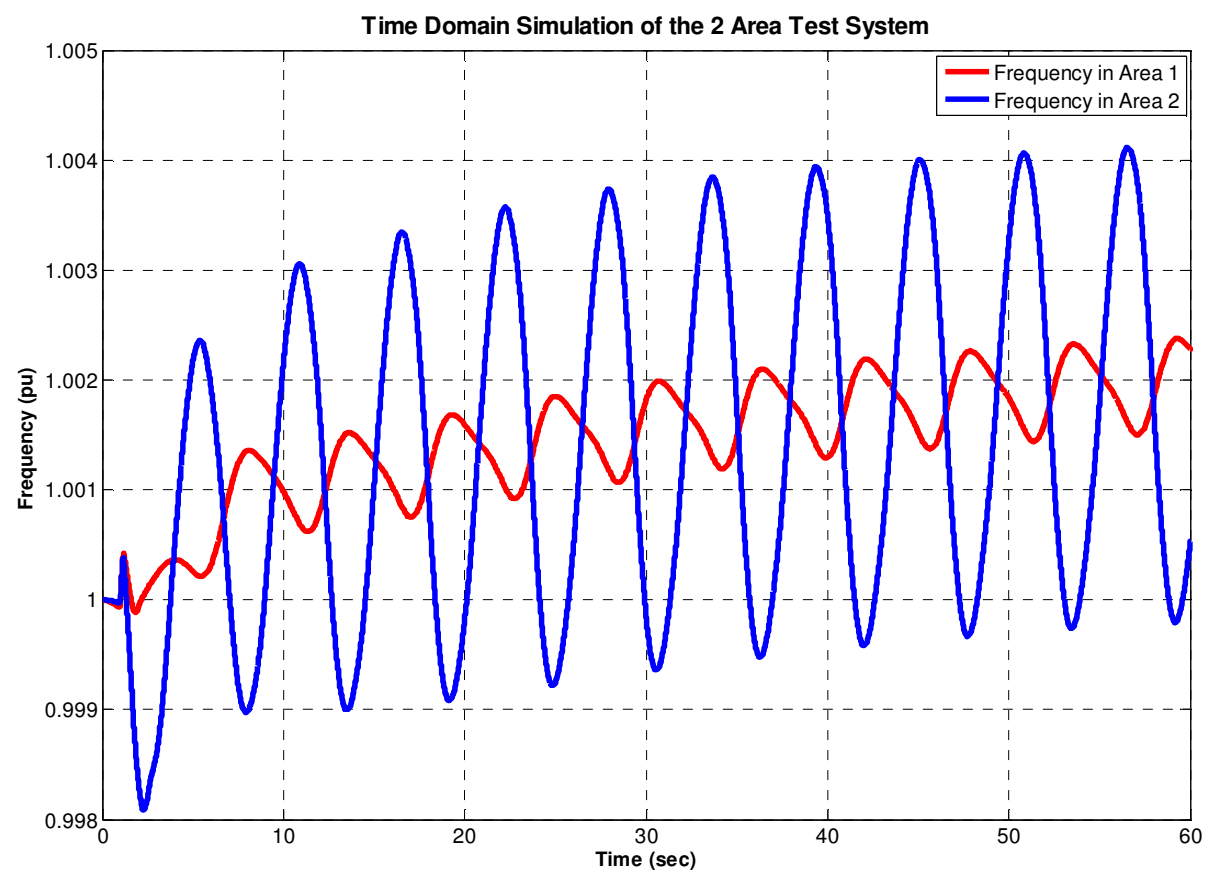

Fig. 4-18: The Frequency of the Two Interconnected Areas of the Test System after a Transmission Line Outage with the Existing Governor Settings 


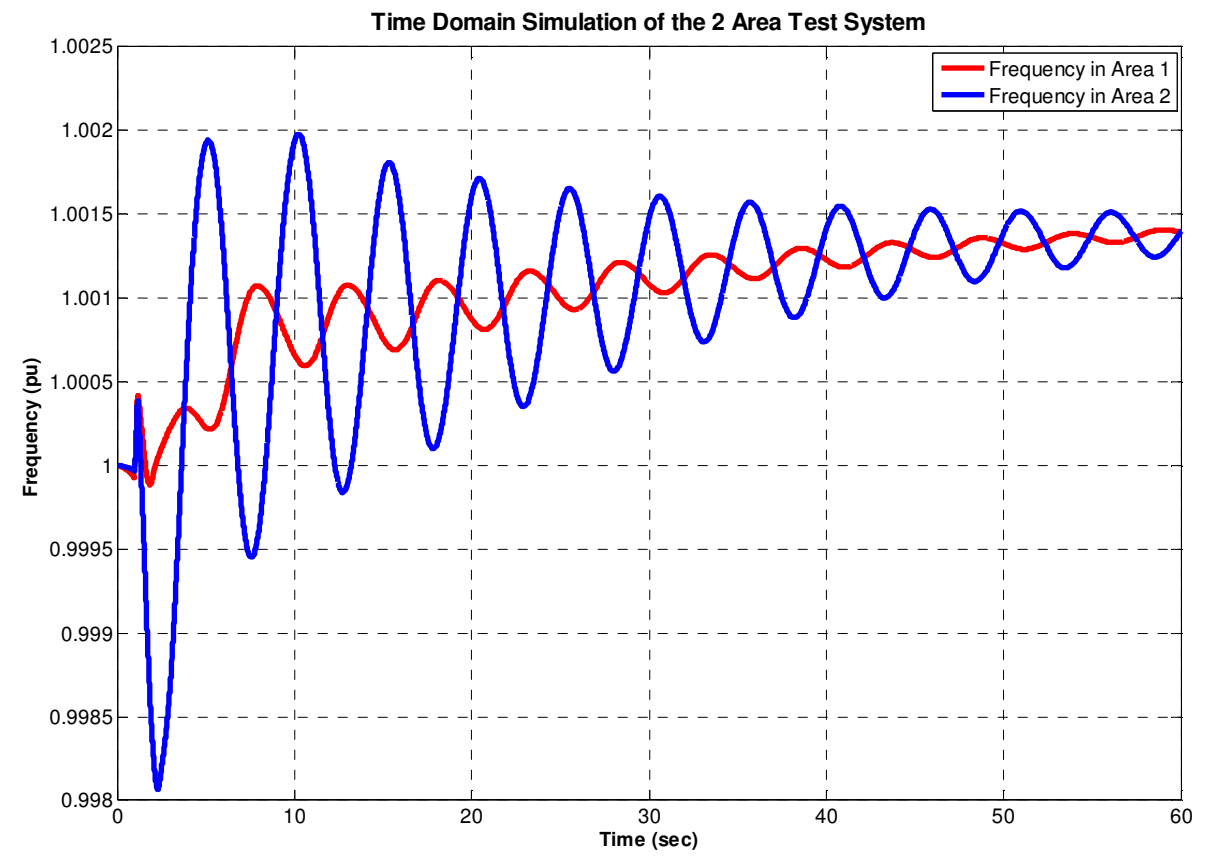

Fig. 4-19: The Frequency of the Two Interconnected Areas of the Test System after a Transmission Line Outage with the Proposed Governor Settings

It can be clearly seen by inspection of time domain simulations that, with the removal of sources of negative damping throughout the system, the overall damping performance of the system for poorly damped low frequency inter area modes is expected to be enhanced.

In simulations of the test system, the behavior of the proposed settings against contingencies (considering the ferquency stability of the system) were also found out to be satisfactory since the system frequency was observed to be stabilized (not restored since the secondary AGC controller, which is out of the scope of this study, is not modeled in the test system) in a period that is approximately equal to 30 seconds. 


\subsection{Karakaya Power Plant Modeling Study}

Karakaya Hydroelectric Power Plant is among the most important electricity generation facilities in the Turkish Power System with six Francis Type hydroelectric turbines each rated at $300 \mathrm{MW}$ and an annual electricity generation of $7354 \mathrm{GWhs}$. Together with Ataturk Hydroelectric Power Plant (8x300MW Francis Turbines), Karakaya Power Plant have a very dominant effect on the frequency control performance of the Turkish Power System since the two power plants satisfy $\sim 14 \%$ of the instantaneous maximum demand $(\sim 30 \mathrm{GW})$. Therefore, the most dominant deteriorating effect on system damping in case of low frequency inter area oscillations is expected from those two power plants.

Similar to Sir Hydroelectric Power Plant, the same construction and electromechanical equipment is utilized in all of the six identical units in Karakaya Hydroelectric Power Plant. Therefore, both the site tests and the parameter tuning study are performed in a single unit. Afterwards, it is assumed that the proposed settings are valid for all the identical units.

The basic system-wide constraints, which consider the system operating philosophy after the ENTSO-E CESA interconnection of Turkish Power System as defined in [4], constitute the expectations from the frequency control performance of a considerable size individual hydroelectric unit from the viewpoint of the TSO, which can be stated as follows:

(i) As stated in [5], due to the high percentage of hydroelectric power plants in Turkey, the hydroelectric power plants should operate so as be stable in island mode even if they operate interconnected to the grid.

(ii) For the step response tests, a settling time comparable with 30 seconds should be achieved .

(iii) The turbine governor systems of the hydroelectric power plants should not deteriorate the damping performance of the system for low frequency inter area power swings. 


\subsubsection{Governor Tuning by Single Machine Infinite Busbar Model}

\subsubsection{Evaluation of the Existing Governor Settings}

In order to evaluate the primary frequency control performance of the hydroelectric units with respect to the previously explained expectations of TEIAS regarding the synchronous interconnected operation, site tests have been performed. As stated before, it is expected to perform the following site tests which have already been explained in 4.2.1.2.:

(i) Island Mode Stability Test

(ii) Step Response Tests

(iii) Frequency Response Tests

Since the defacto mode of operation of the hydroelectric power plants when they are interconnected to the grid is the power control mode with frequency influence, this operational mode has been focused on in the simulation studies. Although Karakaya Power Plant together with Ataturk Power Plant have their specially designed controller structures, the basic principle of the control algorithm is the same with that of the one illustrated in Fig. 3-1.

The computer simulation model is verified by comparing the dynamical behaviour of the model with the site measurements. The existing parameter set corresponding to the operational mode of the governor is illustrated in Table 4-4.

Table 4-4: The Existing Parameter Set

\begin{tabular}{|c|c|c|c|c|c|c|}
\hline Description & $\begin{array}{c}\text { Permanent } \\
\text { Droop }\end{array}$ & $\begin{array}{c}\text { Transient } \\
\text { Droop }\end{array}$ & $\begin{array}{c}\text { Integration } \\
\text { Time Const. } \\
(\mathrm{sec})\end{array}$ & $\begin{array}{c}\text { Power } \\
\text { Control } \\
\text { Time Const. } \\
(\mathrm{sec})\end{array}$ & $\begin{array}{c}\text { Accelerating Path } \\
\text { Time Constant (sec) }\end{array}$ & $\begin{array}{c}\text { Accelerating } \\
\text { Path } \\
\text { Gain }\end{array}$ \\
\hline Parameter & $\mathrm{K}_{\mathrm{dp}}$ & $\mathrm{K}_{\mathrm{h}}$ & $\mathrm{T}_{\mathrm{h}}$ & $\mathrm{T}_{\mathrm{I}}$ & $\mathrm{T}_{\mathrm{D}}$ for $\mathrm{K}_{\mathrm{D}}$ & $\mathrm{K}_{\mathrm{D}}$ \\
\hline Value & $\% 4$ & $\% 12$ & 15 & 15 & 0.5 & 8.5 \\
\hline
\end{tabular}


It is important to note here that all practical speed governor systems utilize dead band blocks for the frequency deviation signal. However, continuous usage of such blocks both significantly deteriorates the frequency control performance of the units and the frequency stability of the grid. Therefore, for the units with significant capacity, such nonlinear blocks should only be used under specific circumstances with the permission and awareness of the TSO. Therefore, it is assumed that the bandwidth of the dead band block is set to zero (i.e., the block is inactive) throughout the simulation studies.

As per the expectations of the TSO, the simulation studies are conducted for the following cases:

(i) Island Mode Simulations

(ii) Step Response Simulations

(iii) Frequency Response Simulations

- Island Mode Simulations (+5\% Load Change)

As stated before, in the island mode simulations, it is assumed that the unit feeds an isolated region from the grid and the only rotating element in the islanded network is the unit itself. Hence, it is clear that the rotor speed of the unit determines the frequency of the isolated network. The behavior of the unit is investigated when there is a sudden significant demand change in the isolated network. It is expected that the unit should be capable of maintaining the frequency stability of the isolated network for such disturbances.

In Fig. 4-20, the output power of the hydroelectric unit is illustrated. It is clear from Fig. 4-20 that the unit is unstable in island mode. The main reason of the initial false response is the fast proportional response of the governor which should be avoided. As stated in [2], a hydroelectric unit should have large transient gain and long resetting time in order to be stable in island mode. 


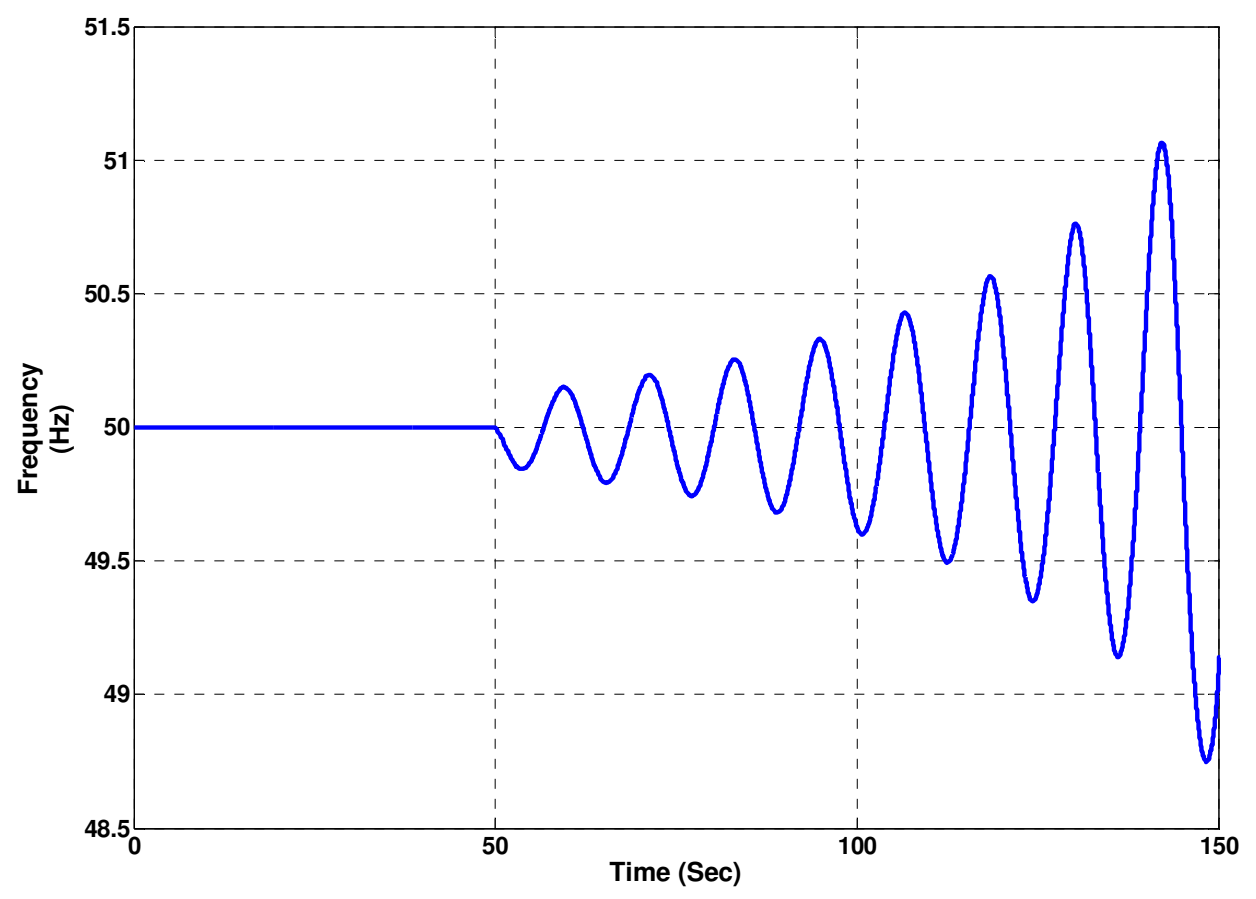

Fig. 4-20: Island Mode Simulation (+5\% Load Change) with the Existing Governor Settings

\section{- $200 \mathrm{mHz}$ Step Frequency Deviation Simulations}

As per the instructions of the UCTE ground rules [4], each control area should provide its share of the total operational reserve in approximately 30 seconds in case of a contingency. The simulations regarding the output power response of the unit against step frequency deviations (simulating the frequency of the system after a severe disturbance) is illustrated in Fig. 4-21.

It can be seen from the simulation results that the settling time of the unit is about 60 seconds, that is well above the maximum limit of the activation of the primary frequency control reserve. Since Karakaya Power Plant has a total capacity of 1800 MW, which correspond to a primary frequency control reserve of $180 \mathrm{MW}$ with the existing governor settings, it is clear that the settling time of the unit is crutial considering the possible conflicts with the central secondary controller which also 
operates so as to maintain both the frequency and the scheduled tie line exchange at the desired values.

In UCTE ground rules, it is also stated that, in addition to activating the primary frequency control reserve in 30 seconds, the unit should also be capable of maintaining its response for 15 minutes in case the frequency is not restored. Since Karakaya HPP also has a considerable reservoir size, all units are capable of maintaining their response for 15 minutes, in case the frequency is not restored or the secondary controller orders them to operate in that fashion, owing to the large potential energy of the stored water in the reservoir.

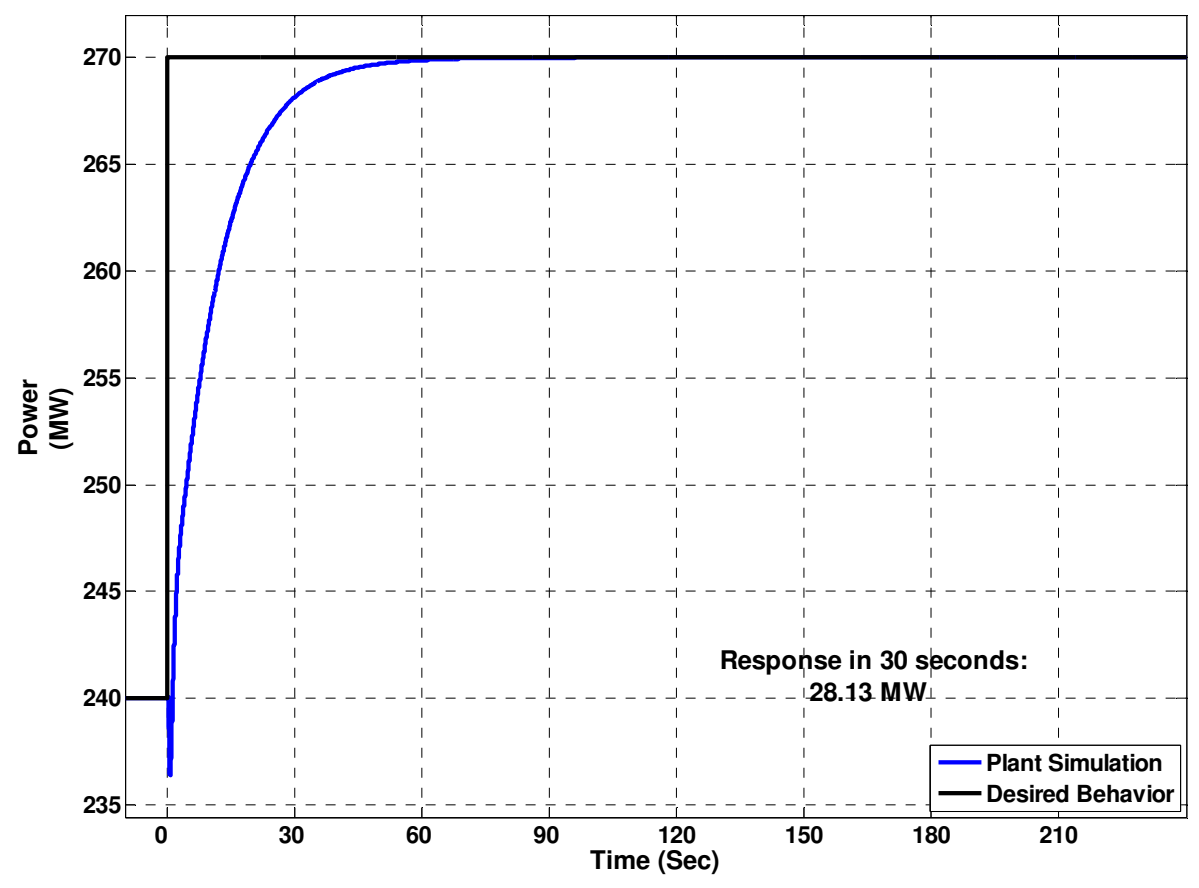

Fig. 4-21: The Response of Mechanical Power against Step Frequency Deviations with the Existing Governor Settings 


\section{- Frequency Response Simulations}

In order to observe the effect of the governor settings on system damping in the frequency bandwidth of the possible inter area oscillations after the interconnection of the Turkish Power System with the ENTSO-E CESA system, the frequency response of the turbine governor system is investigated. The simulations are carried out by injecting a pure sinusoidal frequency signal with an offset value instead of the frequency measurement signal. The simulation results are evaluated by investigating the magnitude and phase of the first harmonic of the steady state output power.

The simulation results of the system against $100 \mathrm{mHz}$ amplitude $0.15 \mathrm{~Hz}$ sinusoidal frequency oscillations is illustrated in time domain in Fig. 4-22. The phasor corresponding to the mechanical power of the machine is plotted in phasor domain in Fig. 4-23 where the $x$ axis (i.e., the reference) is in phase with the damping torque component of the unit (i.e., in phase with $-\Delta w$ ).
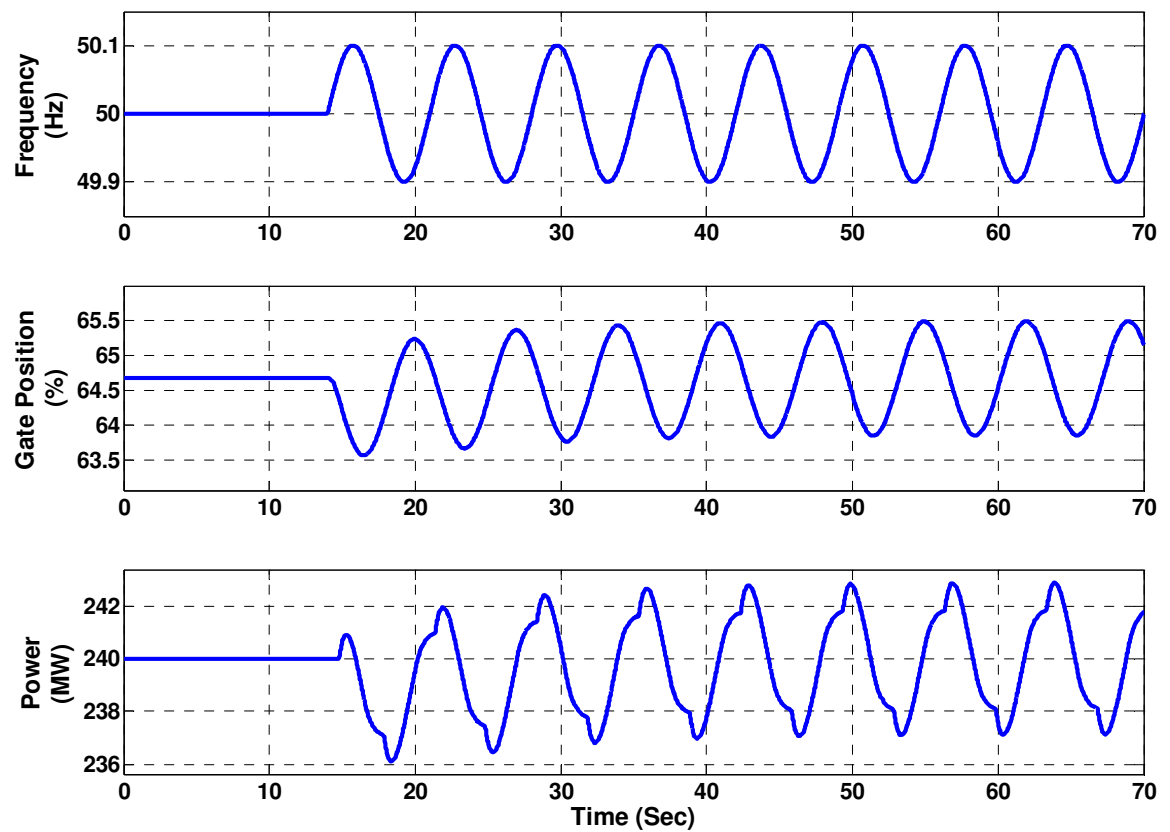

Fig. 4-22: 0.15 Hz Sinusoidal Frequency Oscillations Simulation with the Existing Governor Settings 
As can directly be assessed from Fig. 4-23, the turbine governor of the unit produces a torque component that constitutes a source of negative damping, hence threatening both the frequency stability of the system and the steady state stability of the system.

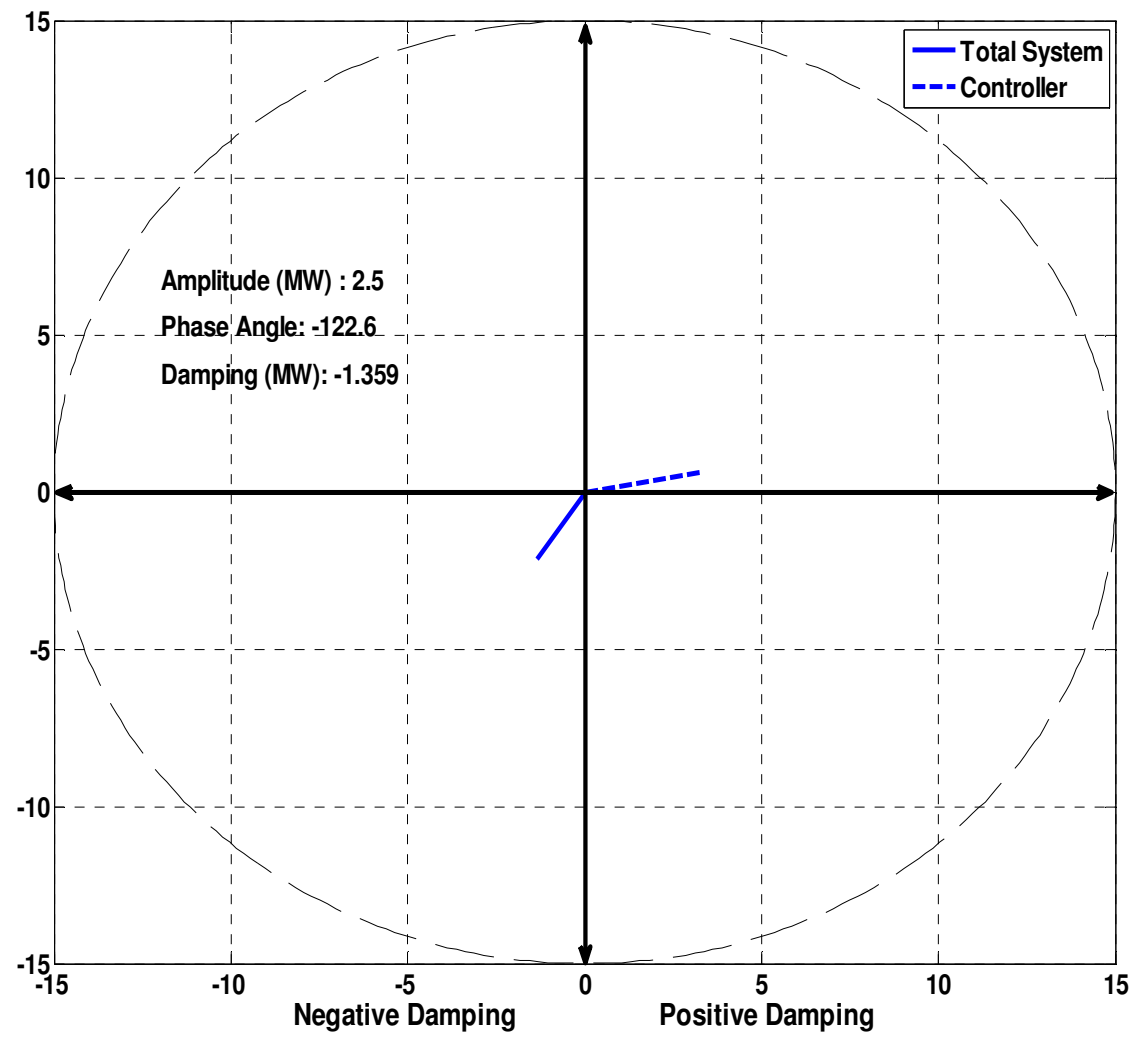

Fig. 4- 23: The Phasor Diagram of the Mechanical Power Response of the Unit against Sinusoidal Frequency Input with the Existing Governor Settings

Evaluating the primary frequency control performance of the sample unit, with the existing governor settings, from this point of view, it is observed that,

(i) The existing governor settings of the hydroelectric power plant are unstable in island mode. 
(ii) The settling time of the mechanical power response against step frequency deviations for the hydroelectric unit was about 60 seconds.

(iii) The mechanical response of the unit would deteriorate the system damping in case of possible sustained (or growing) low frequency inter area oscillations $(\sim 0.15 \mathrm{~Hz})$ as explained in the part 2.5.2 (i.e., in Fig. 229).

\subsubsection{The Proposed Governor Settings}

As per the expectations of the TSO, the simulation studies regarding the retuning of the governor are conducted with the following constraints:

(i) The unit should be stable in island mode as stated in [5].

(ii) The settling time of the unit against step frequency deviations should be comparable with 30 seconds (If possible together with (iii)).

(iii) The turbine governor of the unit should not deteriorate the system damping performance against inter area power swings (If this is not possible due to physical constraints, the smallest possible adverse effect should be ensured).

The proposed parameter set, corresponding to the operational mode of the governor and obtained as a result of the simulation results those are to be explained, is tabulated in Table 4-5.

Table 4-5: The Proposed Parameter Set

\begin{tabular}{|c|c|c|c|c|c|c|}
\hline Description & $\begin{array}{c}\text { Permanent } \\
\text { Droop }\end{array}$ & $\begin{array}{c}\text { Transient } \\
\text { Droop }\end{array}$ & $\begin{array}{c}\text { Integration } \\
\text { Time Const. } \\
(\mathrm{sec})\end{array}$ & $\begin{array}{c}\text { Power } \\
\text { Control } \\
\text { Time Const. } \\
(\mathrm{sec})\end{array}$ & $\begin{array}{c}\text { Accelerating Path } \\
\text { Time Constant (sec) }\end{array}$ & $\begin{array}{c}\text { Accelerating } \\
\text { Path } \\
\text { Gain }\end{array}$ \\
\hline Parameter & $\mathrm{K}_{\mathrm{dp}}$ & $\mathrm{K}_{\mathrm{h}}$ & $\mathrm{T}_{\mathrm{h}}$ & $\mathrm{T}_{\mathrm{I}}$ & $\mathrm{T}_{\mathrm{D}}$ for $\mathrm{K}_{\mathrm{D}}$ & $\mathrm{K}_{\mathrm{D}}$ \\
\hline Value & $\% 8$ & $\% 12$ & 15 & 15 & 0.5 & 8.5 \\
\hline
\end{tabular}


Similar to the case for Sir Hydroelectric Power Plant, since the basic motivation of the retuning study is to remove the component of the mechanical torque that deteriorates the system damping performance, the phase characteristics of the transfer function of the governor and turbine is focused on in the first step. However, in the case of Karakaya Hydroelectric Power Plant, where the hydroelectric units with very long water starting times, the desired phase lead is impossible to be achieved with satisfactory settling times against contingencies. Therefore, since the basic function of the speed governors is to balance the generation and the demand, the optimum parameter set turns out to be the one where the magnitude of the undesired torque component is minimized.

The selected parameter set is checked for stability in island operation. In case this criterion is not satisfied, another parameter set satisfying the above explained criteria is assessed.

As stated before, a nonlinear hydroelectric turbine model is utilized in this study. However, since the effect of the dynamical system in a certain frequency range is investigated, analysis of the first harmonic of the mechanical power response provides sufficient information about the behavior of the dynamic system as it is the case for linear systems. According to the simulation results, no single parameter set satisfying acceptable settling times against step frequency deviations could be reached together with not deteriorating the damping performance of the system. Therefore, the objective of the tuning study was updated as reaching a parameter set which satisfies island mode stability with the fastest settling time against step frequency deviations. In addition, the magnitude-wise minimization of the mechanical power which deteriorates the system damping performance is also desired.

Therefore, only a modification in the permanent droop setting in the governor is made to reduce the amount of primary frequency control reserve of the unit. By this approach, both the settling time of the unit gets closer to 30 seconds with the parameter sets satisfying the stability criterions and the total amount of primary 
frequency control reserve allocated to hydroelectric power plants decreases which does not cause violation of the (n-1) rule for Turkish Power System in interconnected operation with ENTSO-E CESA System, due to the decreased reserve requirement of the system as a benefit of the interconnected operation.

\section{- Island Mode Simulations (+5\% Load Change)}

The island mode stability simulations are carried out in a similar manner to the ones in 4.2.1.2. and the simulation results are illustrated in Fig. 4-24. From the island mode simulations, it is concluded that the unit is stable in island mode of operation.

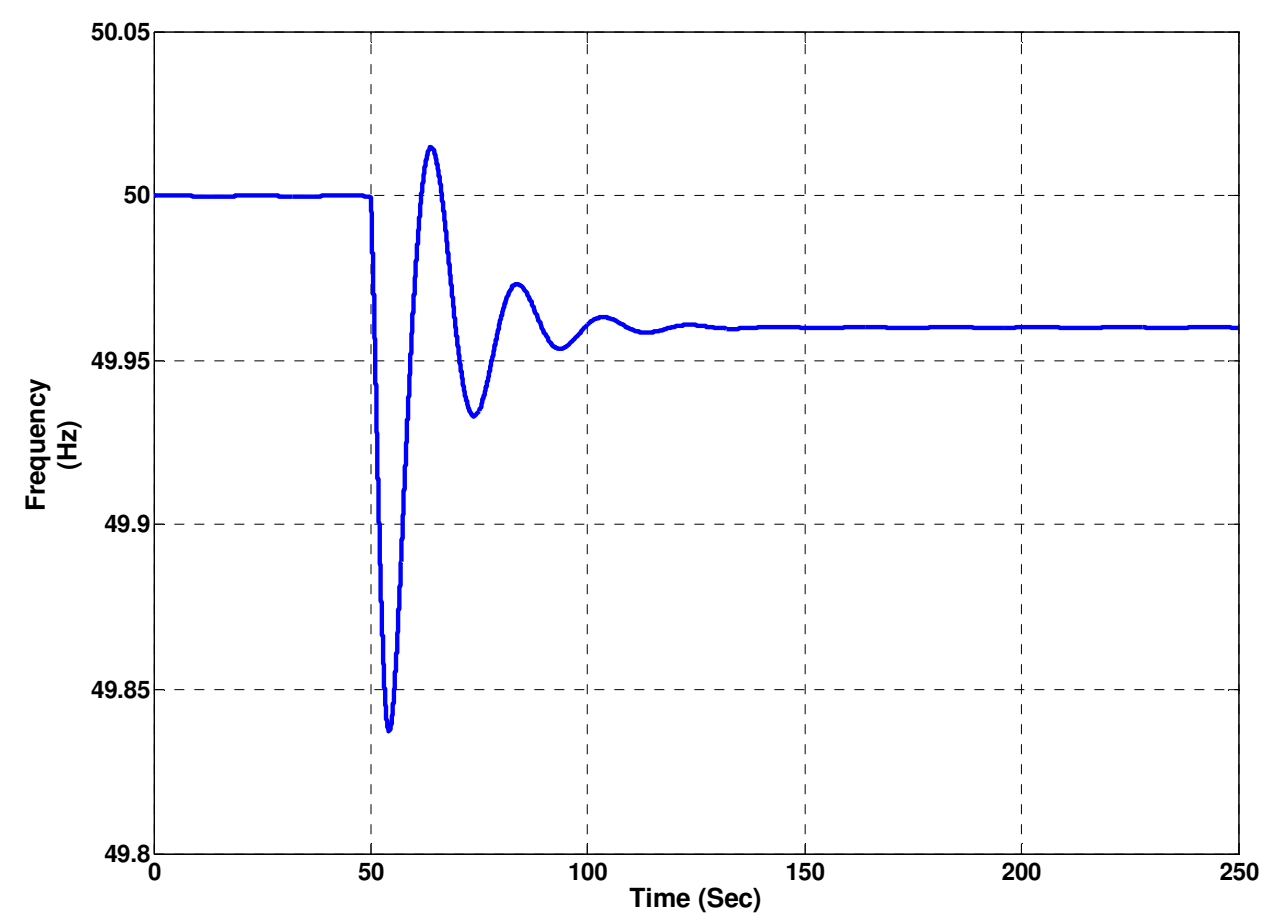

Fig. 4-24: Island Mode Simulation (+5\% Load Change) with the Proposed Governor Settings

- $200 \mathrm{mHz}$ Step Frequency Deviation Simulations

Karakaya Hydroelectric Power Plant, together with Ataturk Hydroelectric Power Plant, has a considerable effect on the frequency stability of the Turkish Power 
System. Therefore, it is expected that the settling time of the individual units against step frequency deviations should be comparable with 30 seconds to avoid unintended power transfer to the ENTSO-E CESA System in case of severe incidences, since the frequency will not be restored in a short time interval causing the unit to continue responding. It is important to note here that this phenomenon may cause conflicts with the central secondary control action since the total amount of primary frequency control reserve allocated to the hydroelectric power plants is significantly large.

In Fig. 4-25, the corresponding simulation results with the proposed governor parameters are plotted. As can directly be assessed from Fig. 4-25, the settling time of the mechanical power against step frequency disturbances is comparable with 30 seconds.

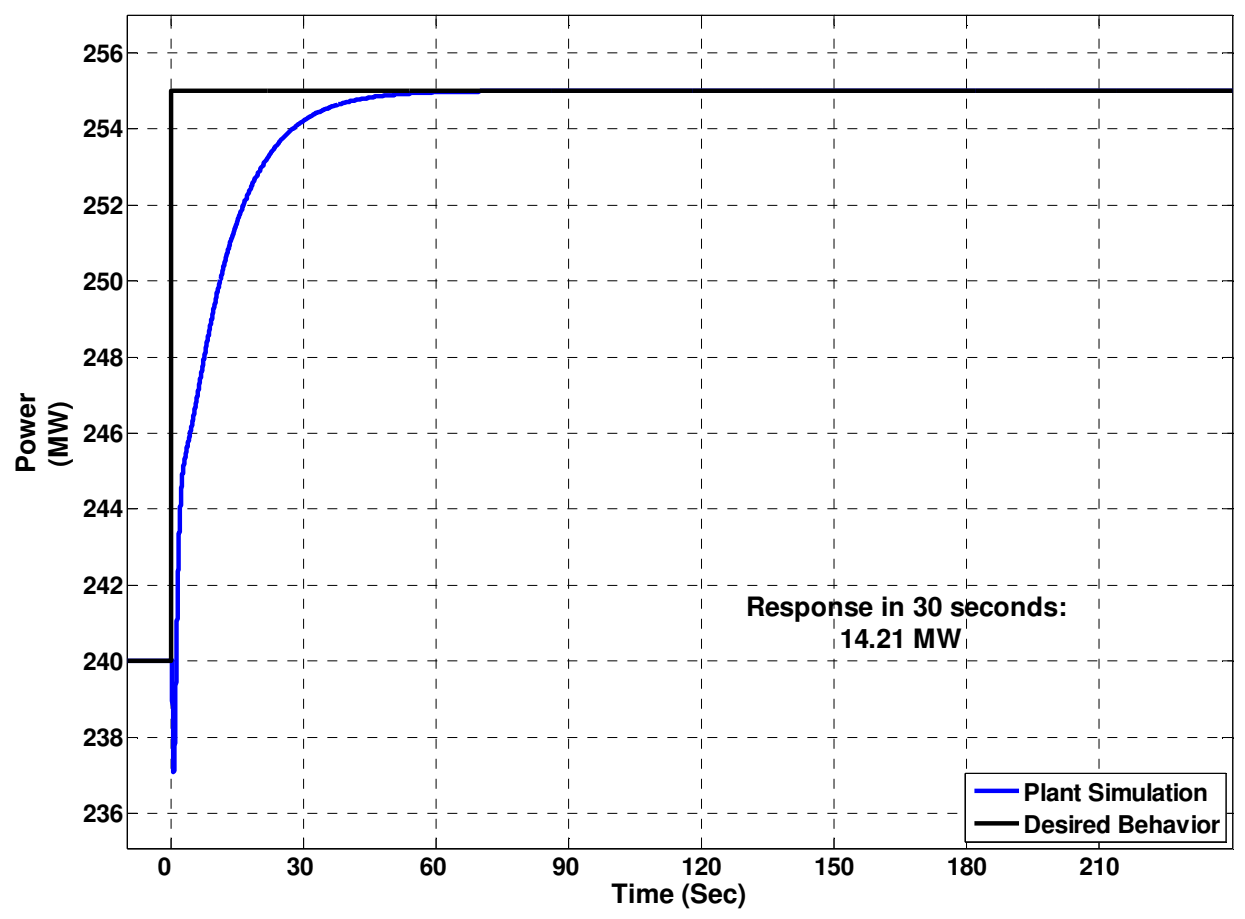

Fig. 4-25: The Response of Mechanical Power against Step Frequency Deviations with the Proposed Governor Settings 


\section{- Frequency Response Simulations}

The simulation results of the system against $100 \mathrm{mHz}$ amplitude $0.15 \mathrm{~Hz}$ sinusoidal frequency oscillations is illustrated in time domain in Fig. 4-26. The phasor corresponding to the mechanical power of the machine is plotted in phasor domain in Fig. 4-27. As can directly be assessed by comparing Fig. 4-27 with Fig. 4-23, the turbine governor of the unit produces a torque component that constitutes a source of negative damping. However, since the magnitude of the torque component deteriorating the damping performance of the system is decreased, a better parameter set considering the frequency stability and the steady state stability of the system is achieved.
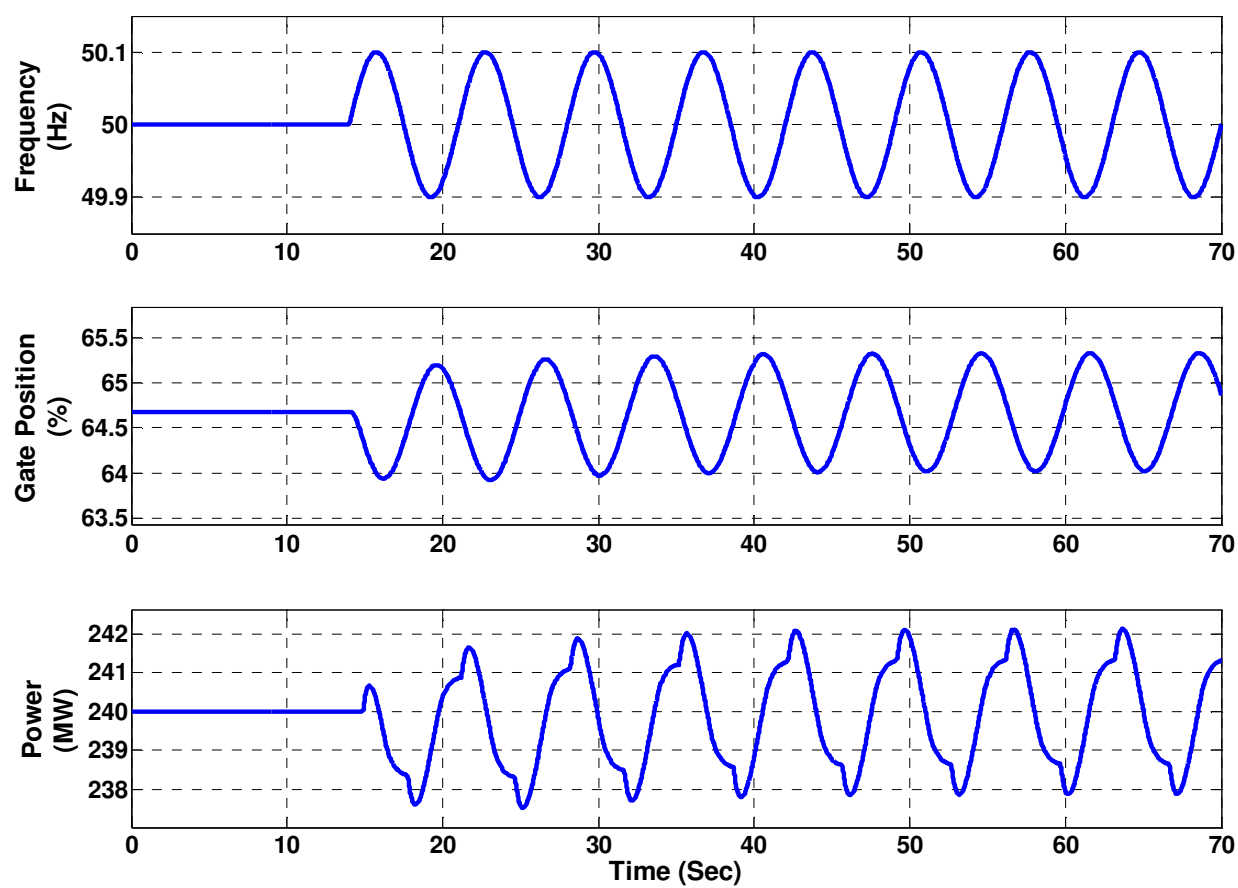

Fig. 4-26: 7 Sec Sinusoidal Frequency Oscillations Simulation with the Proposed Governor Settings 


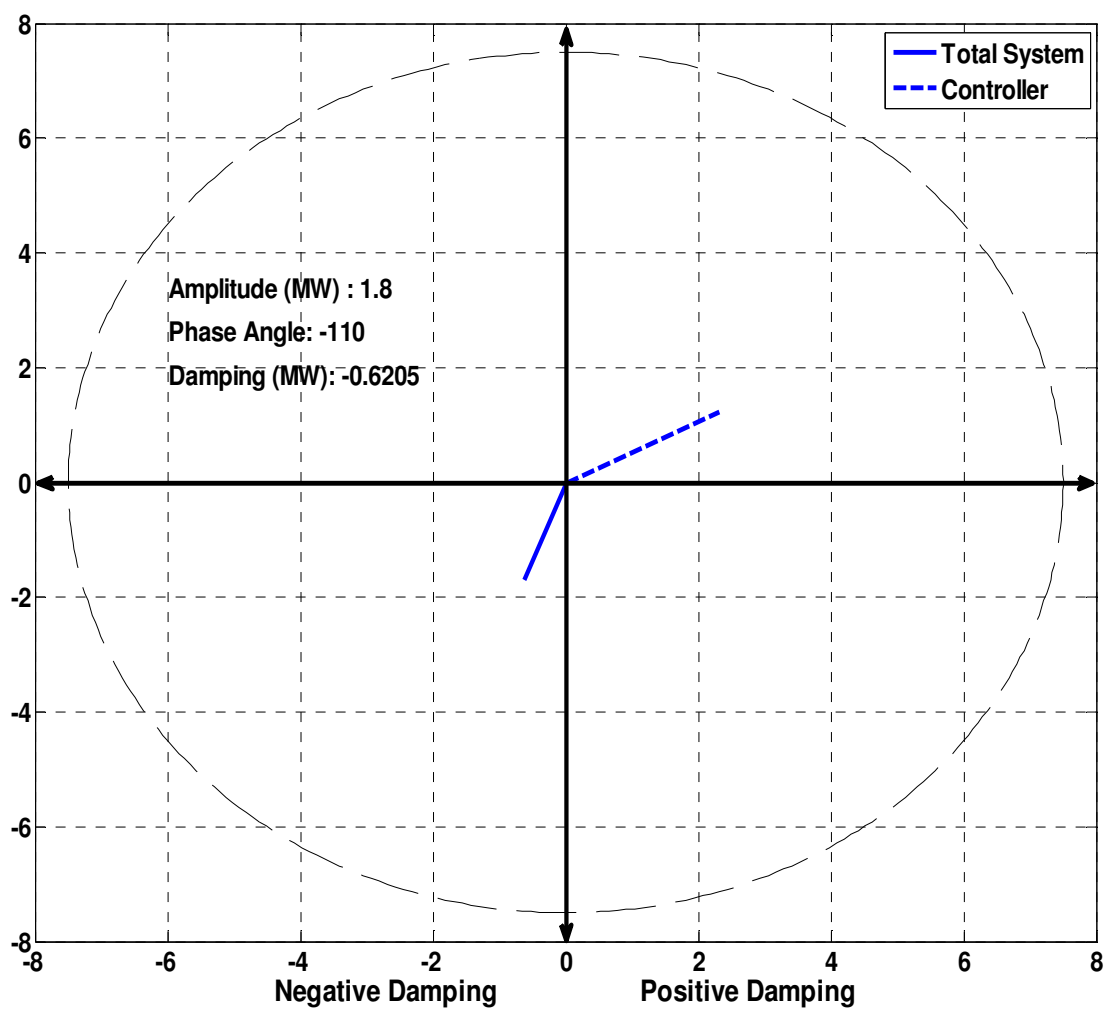

Fig. 4-27: The Phasor Diagram of the Mechanical Power Response of the Unit against Sinusoidal Frequency Input with the Proposed Governor Settings

\subsubsection{Assessment of Effectiveness of the Proposed Governor Settings in} Multi Machine Network

Similar to the procedure followed in the part 4.2.1.3., the tuning study of the governor of the hydroelectric unit is performed in a single machine infinite busbar model. Since the mechanical power of the turbine is of major interest in the tuning studies, the generator and exciter of the unit are not modeled, which are the initiators of the possible poorly damped inter area oscillations. This very low frequency electromechanical phenomenon turns into a generation-load imbalance problem in case of sustained (or very poorly damped) inter area swings, as it is also the case in this study. 
Similar to the method utilized in the part 4.2.2., in order to demonstrate the effect of the retuned parameter set of the hydroelectric unit to the damping performance of the system against low frequency $(\sim 0.15 \mathrm{~Hz})$ inter area oscillations, Kundur's Two Area Power System in [14] is utilized. The network topology, the load flow (dispatch) scenario together with the other assumptions regarding the controller structures of the power plants and the modifications made in the original network proposed in [14], are also the same with those utilized in the Section 4.2.2.

Similar to the case investigated in Section 4.2.2., Area 1 is assumed to transfer 400 MW to Area 2 in order to create a weak system configuration from the viewpoint of small signal rotor angle stability, in addition to simulating a permanent fault causing outage in one of the two transmission lines between the two areas. This contingency ends up with a weaker transmission topology assuming that the scheduled transaction between the two areas is kept constant after the incident.

It has been observed from the simulations that the generators both in Area 1 and in Area 2 oscillate coherently and hence represent the expected mode shapes of the Turkish Power System [16] and ENTSO-E CESA System after interconnection. Fig. 4-28 illustrates the frequency of the two power systems after the outage of one of the transmission lines with the existing and the proposed governor settings of the hydroelectric unit of interest after the outage of one of the transmission lines.

Throughout the tuning study, unlike the case for the Sir Hydroelectric Power Plant, the only contribution to the system damping performance was lowering the magnitude of the torque component deteriorating the system damping performance. Therefore, only a slight improvement could be achieved in the simulations regarding the overall damping performance of the system for poorly damped low frequency inter area modes. It is important to note here that the system was found to be well damped in both simulation scenarios.

In addition, in simulations of the test system, the behavior of the proposed governor settings against contingencies (considering the ferquency stability of the system) 
were also found out to be satisfactory since the system frequency was observed to be stabilized (not restored since the secondary AGC controller, which is out of the scope of this study, is not modeled in the test system) in a period that is approximately equal to 30 seconds. However, with the existing governor settings, the problem of unintentional power flow in the case of contingencies was observed in the simulations.

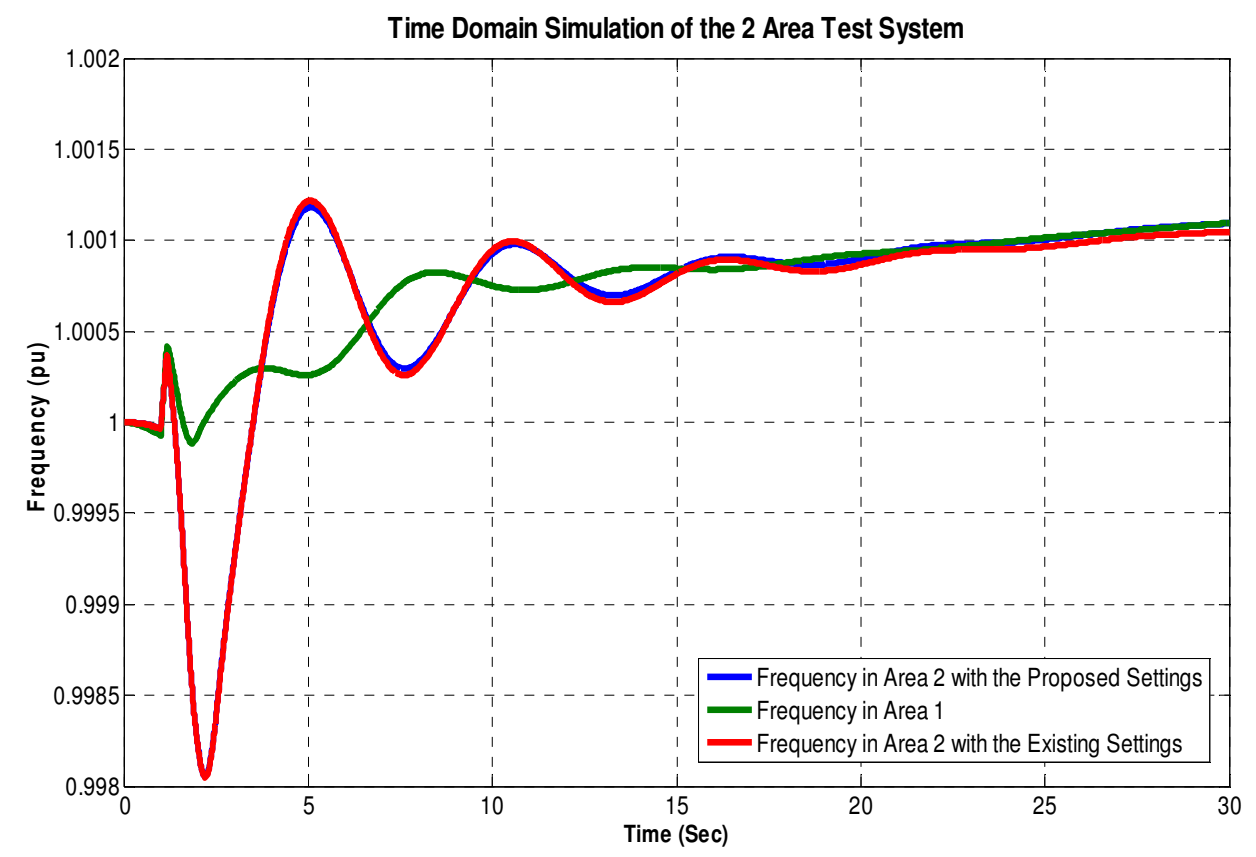

Fig. 4-28: The Frequency of the Two Interconnected Areas of the Test System after a Transmission Line Outage with the Proposed Governor Settings

\subsection{System Wide Effect of the Retuned HPPS}

After it is concluded that the proposed settings are suitable for network operation, the effectiveness of the proposed settings in terms of removal of the negative damping sources in the system are simulated. The simulation study is carried out by modification of the governor settings of the large scale hydroelectric power plants one by one with the proposed ones in [21]-[29] in order to better investigate the 
effect of all hydroelectric power plants in the priority list defined in [6], using the global system data (i.e., the reduced ENTSO-E CESA System and the comprehensive Turkish Power System data).

\subsubsection{Load Flow Scenario}

In Fig. 2-29 and Fig. 2-30, it was shown that once the interconnected operation of the Turkish Power System and the ENTSO-E CESA System is established, inter area oscillations as a result of the poorly damped modes of the system were prone to occur depending on the system topology and the dispatch scenario. It was also stated that the worst case scenario was found out to occur in the minimum loading condition of both the Turkish Power System and the ENTSO-E CESA System.

However, as well known, hydroelectric power plants generally operate as peaker plants, which means that they are operational in certain periods of the day since the start up time of the hydroelectric power plants is quite short and the start up costs of them are quite low. Therefore, a realistic operational scenario is selected where the dynamical behavior of major hydroelectric power plants dominate the dynamical behavior of the Turkish Power System.

Hence, the maximum loading condition of the two systems (ENTSO-E CESA System demand is $329 \mathrm{GW}$ and Turkish Power System demand is $30 \mathrm{GW}$ ) is simulated where poor damping performance is expected due to the high loading conditions. In addition, the PSSs of all the major power plants are turned off in order to focus only on the effect of the removal of sources of negative damping for low frequency inter area modes and a market situation where Turkey exports $1000 \mathrm{MW}$ to Italy over the Balkan countries is simulated in order to create a weak transmission scenario. It is important to note that all significant hydroelectric power plants are in service due to peak loading conditions. 


\subsubsection{Simulation Results}

It was previously explained that the Ataturk (8x300) and Karakaya (6x300) power plants satisfy the $14 \%$ of the peak demand of the Turkish Power System at peak loading conditions and hence the dynamic characteristics of their mechanical controllers have a dominant role in the overall frequency control dynamics of the control area, namely the Turkish Power System. Fig. 4-29 illustrates the frequency of Turkish Power System after the outage of a 1200 MW power plant in Spain, when Ataturk and Karakaya Hydroelectric Power Plants (4200 MW total capacity) are retuned as explained in [26] and [27] (in the red trend). Note that the blue trend in Fig. 4-29 illustrates the case with the existing governor settings of the hydroelectric power plants in the priority list [6].

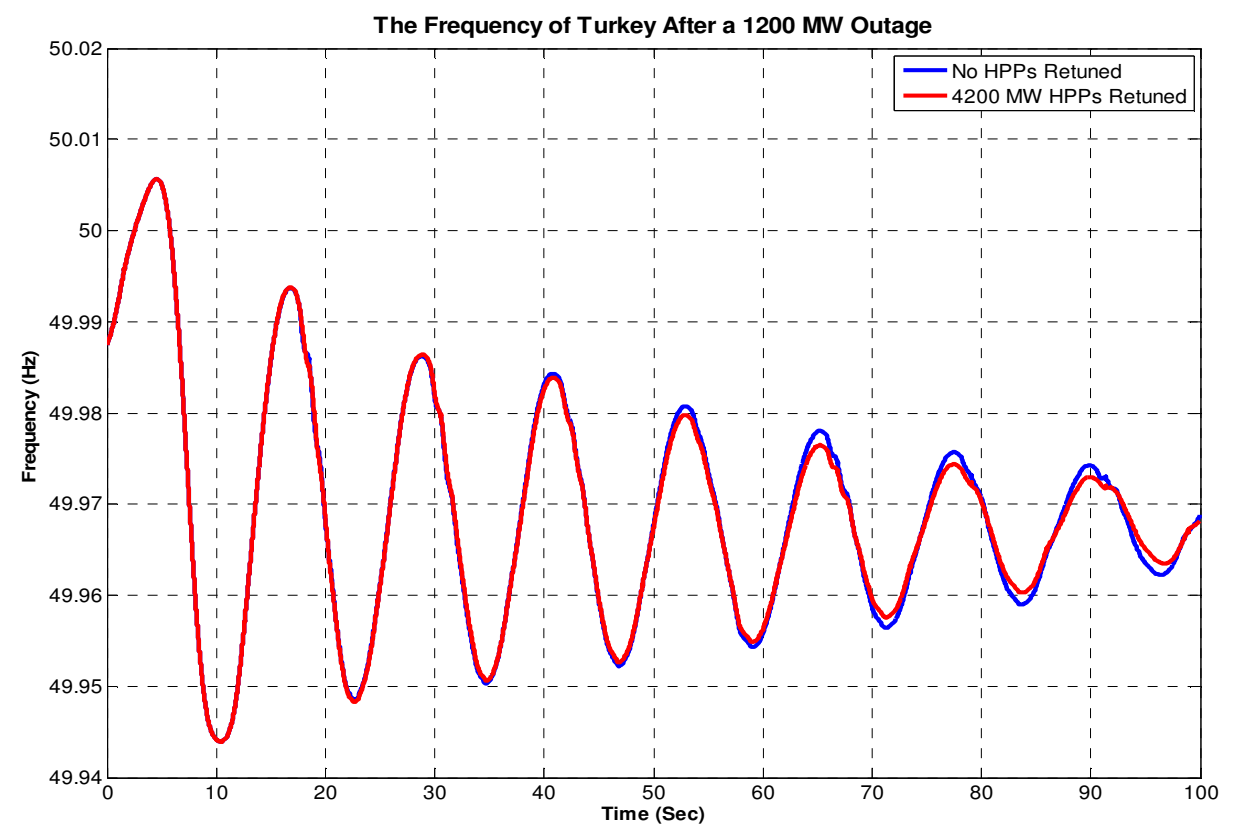

Fig. 4-29: The Frequency of the Turkish Power System after a 1200MW Outage

As can be directly assessed from Fig. 4-29, the damping performance of the Turkish Power System against the inherent low frequency inter area modes is enhanced, since 
the two major hydroelectric power plants have a dominant role in the overall frequency control performance of the Turkish Power System.

The case where the amount of the removal of sources of negatice damping is increased is illustrated in Fig. 4-30. Fig 4-30 illustrates the frequency of Turkish Power System after the outage of a 1200 MW power plant in Spain when all hydroelectric power plants in the priority list in [6] are retuned according to [22][29], except Keban Hydroelectric Power plant which is in the scope of rehabilitation work and assumed to be operating in the power control mode, hence insensitive to frequency changes (in the black trend).

It can be directly observed from Fig. 4-30, by comparing the three trends, that as the amount of the removal of sources of negative damping throughout the system is increased, the system damping performance is enhanced. This fact shows that the prevention of the amplification of the low frequency electromechanical oscillations due to the action of speed governing structures in hydroelectric power plants is an essential task to be completed before interconnection of the Turkish Power System with the ENTSO-E CESA System. In addition, this study constitutes a preliminary work to be completed prior to the studies related to the damping measures against the poorly damped modes such as the retuning of the PSSs of major power plants and controller modification of the static power system devices (i.e., SVCs, STATCOMs, etc.). 


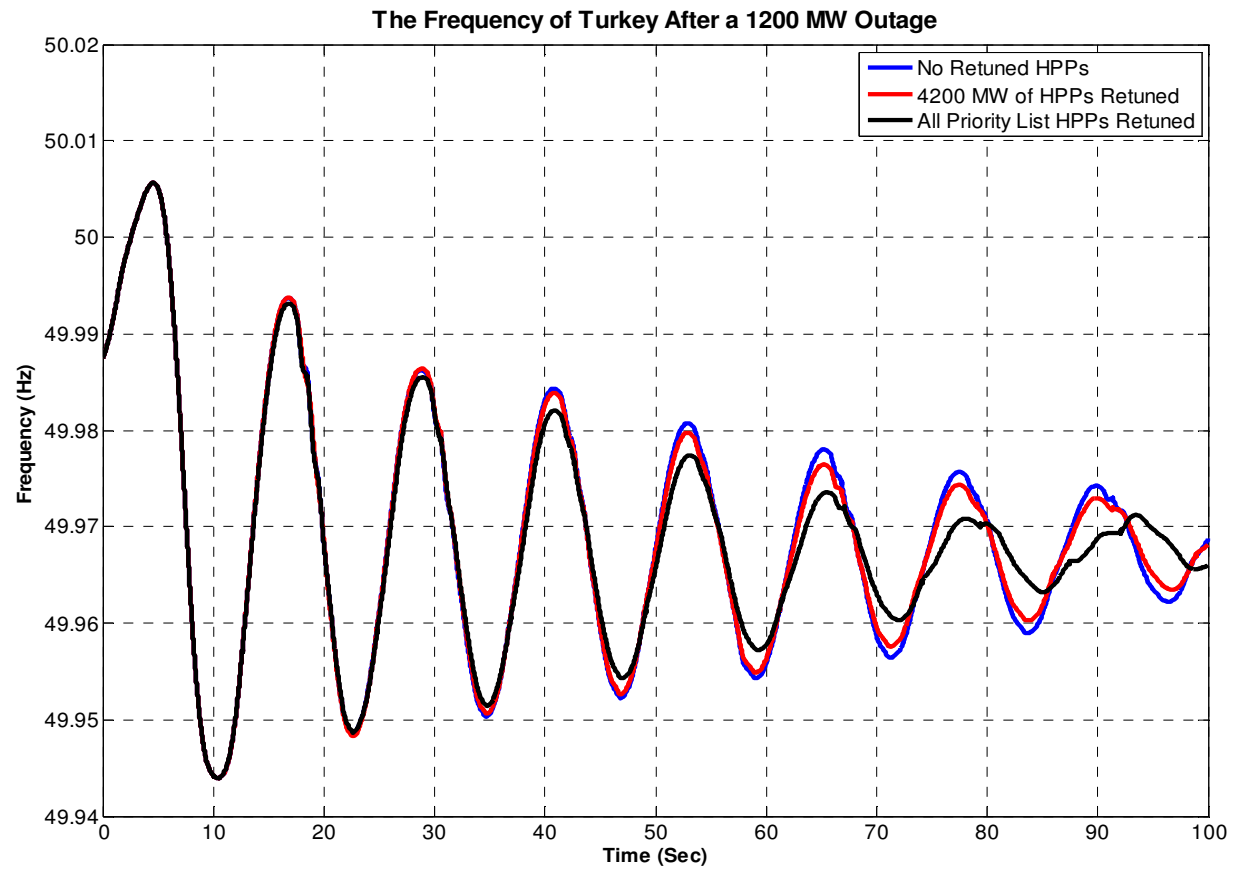

Fig. 4-30: The Frequency of the Turkish Power System after a 1200MW Outage 


\section{CHAPTER 5}

\section{CONCLUSIONS AND FUTURE WORK}

This study investigates the effects of governor settings of large size hydroelectric power plants on the expected inter area power swings after the interconnected operation of the Turkish Power System with the ENTSO-E CESA System.

Since the expected frequency range of inter area swings $(\sim 0.15 \mathrm{~Hz})$ after the interconnection of the two systems is within the frequency range of speed governing structures of the power plants (provided that magnitude of the frequency oscillations increase over a certain limit so as to exceed the unintentional insensitivity (similar to hysteresis phenomenon), which is about $10 \mathrm{mHz}$, in mechanical control systems), turbines in large size hydroelectric power plants are particularly shown to be the possible sources of negative damping, using the nonlinear simulation models. As the most important conclusion of this study, it is shown that with the removal of sources of negative damping for the frequency of expected poorly damped modes for the synchronous interconnected operation of the Turkish Power System and ENTSO-E CESA System by the proposed governor tuning methodology proposed in the study, the damping performance of the system is enhanced significantly.

The model parameter tuning study with regard to the objectives of enhancing system damping to prevent contribution to inter area power swings and acceptable LFC performance from the viewpoint of the TSO, which concerns acceptable response time and hence minimization of unintended power transfer through tie lines, is performed for two sample hydroelectric power plants. The two sample power plants are represented to explain the two different methodologies followed in the governor 
tuning studies regarding the major hydroelectric power plants in the Turkish Power System. In addition, the effect of retuned governor settings of the sample hydroelectric power plants on the frequency stability is shown with case studies concerning system damping and acceptable LFC performance by utilizing a representative multi-machine power system model.

Finally, the overall effect of retuned hydroelectric power plants in the priority list [6] is shown and it is concluded that with the removal of possible sources of negative damping throughout the system, the damping performance hence the small signal stability and the security of the system are enhanced.

The main conclusions reached throughout this study regarding the operation of the hydroelectric power plants, considering the frequency stability of the system after synchronous interconnected operation of the Turkish Power System and the ENTSOE CESA System can be stated as:

(i) For those hydroelectric units with relatively small water starting time such as the case for the Sir Hydroelectric Power Plant, the governor settings which are stable in island mode and at the same time do not deteriorate the system damping performance with acceptable settling time against frequency deviations can be achieved by the tuning methodology proposed in Section 4.2.1.3.

(ii) For those hydroelectric units with larger water starting times such as the case for the Karakaya Hydroelectric Power Plant, the governor settings which are stable in island mode and at the same time do not deteriorate the system damping performance with acceptable settling time against frequency deviations can not be achieved due to physical constraints. Therefore, since such units essentially have considerable capacity, the contribution of such units to the frequency stability of the system is inevitable, which means that such hydroelectric units should be operated with the governor settings which are stable in island mode with acceptable settling time against frequency deviations. The magnitude of 
the mechanical torque component, which deteriorates the system damping performance against inter area oscillations, created by the speed governing structures of such units, can be minimized via decreasing the amount of the primary frequency control reserve assigned to such units by increasing the permanent droop setting of the governors of the corresponding units. Note that this approach will not cause the violation of the (n-1) rule for the Turkish Power System due to the decreased primary reserve requirement after interconnected operation with the ENTSO-E CESA System. Such a governor tuning approach is explained in Section 4.3.1.2. in detail.

(iii) With the removal of possible sources of negative damping throughout the system, by the proposed governor tuning methodologies proposed in (i) and (ii), the damping performance hence the small signal stability and the security of the system are enhanced as shown in Section 4.4.

After presenting the effects of retuned hydroelectric power plants' governor settings on the system damping performance (i.e. the effect of the removal of possible sources of negative damping), the analysis of the effects of other counter measures that may have positive effects on damping of low frequency inter area oscillations can investigated in future, since the TSOs are expected to satisfy the system wide stability criterions so as to guarantee the stable operation of the grid before and after interconnection of the two systems. In addition, such counter measures are also useful for improving the power transfer capacity of the control areas since they will improve the dynamic stability of the system. Those protective damping measures against inter area oscillations may be broadly defined as follows:

(i) Retuning of the PSSs of the considerable size power plants in Turkey so as to damp $0.15 \mathrm{~Hz}$ inter area oscillations, which is an essential preliminary work to be completed before interconnection.

(ii) Modification of the STATCOMs in order to enhance the system damping performance by varying voltage at the busbars where they are coupled, in phase with the frequency oscillations caused by the expected inter area 
modes. Hence the consumed power by the loads connected to the same busbars is also varied so as to damp frequency swings.

(iii) Modification of the SVCs in order to enhance the system damping performance by varying voltage at the busbar where they are coupled, in phase with the frequency oscillations caused by the expected inter area modes. Hence the consumed power by the loads connected to the same busbars is also varied so as to damp frequency swings.

(iv) Installation of braking resistors in order to enhance the system damping performance by varying the consumed power by the resistors out of phase with the frequency oscillations caused by the expected inter area modes. 


\section{REFERENCES}

[1] J. Machowski, J.W. Bialek, J.R. Bumby, Power System Dynamics: Stability and Control, New York: Wiley, 2008.

[2] P. Kundur, Power System Stability and Control, New York: McGraw-Hill, 1994.

[3] IEEE/CIGRE Joint Task Force on Stability Terms and Definitions, "Definitions and Classification of Power System Stability," IEEE Transactions on Power Systems, vol.19, pp. 1387-1399, May 2004.

[4] Union for the Coordination of Transmission of Electricity, UCTE Operation Handbook, Union for the Coordination of Transmission of Electricity, June 2004. [Online]. Available:

http://www.entsoe.eu/index.php?id=57\&no_cache=1\&sword_list[]=handbook. [Accessed May 31, 2010].

[5] M. E. Cebeci, U. Karaagac, O. B. Tor and A. Ertas , "The Effects of Hydro Power Plants' Governor Settings on the Stability of Turkish Power System Frequency “ ELECO Conf, Bursa, Turkey, Dec. 2007.

[6] "Complementary Studies for the Synchronization of the Turkish Power System with the UCTE Power System”, UCTE, May 2007.

[7] M. E. Cebeci, “The Effects of Hydro Power Plants' governor Settings on the Turkish Power System Frequency", Ms Thesis, Middle East Technical University, Feb. 2008. 
[8] E. De Jaeger, N. Janssens, B. Malfliet, F. Van De Meulebroeke, "Hydro Turbine Model for System Dynamic Studies," IEEE Transactions on Power Systems, vol. 9, no. 4, pp. 1709-1715, Nov. 1994.

[9] F. R. Schleif AND A. B. Wilbor, "The Coordination of Hydraulic Turbine Governors for Power System Operation," IEEE Transactions on Power Apparatus and Systems, vol. Pas-85, no.7, pp. 750-758, July 1966.

[10] F. R. Schleif AND J. H. White, "Damping for the Northwest - Southwest Tieline Oscillations-An Analog Study," IEEE Transactions on Power Apparatus and Systems, vol. Pas-85, no. 12, pp. 1239-1247, December 1966.

[11] F. R. Schleif AND G. E. Martin, "Damping of System Oscillations with a Hydrogenerating Unit," IEEE Transactions on Power Apparatus and Systems, vol. Pas-86, no.4, pp. 438-442, April 1967.

[12] IEEE Working Group on Prime Mover and Energy Supply Models for System Dynamic Performance Studies, "Hydraulic Turbine and Turbine Control Models for System Dynamic Studies," IEEE Transactions on Power Systems, vol. 7, no. 1, pp. 167-179, Feb. 1992.

[13] IEEE Committee Report, "Dynamic Models for Hydro Turbines in Power System Studies," IEEE Transactions on Power Apparatus and Systems, 1973.

[14] M. Klein, G. J. Rogers, P. Kundur, "A Fundamental Study of Inter-Area Oscillations in Power Systems," IEEE Transactions on Power Systems, vol. 6, no. 3, pp. 914-921, Aug. 1991.

[15] F. P. Demello AND C. Concordia, "Concepts of Synchronous Machine Stability as Affected by Excitation Control," IEEE Trans. Power Apparatus and Systems, vol. Pas 88, no.4, pp. 316-329, Apr. 1969. 
[16] H. Breulmann, E. Grebe, M. Lösing, W. Winter, R. Witzmann, P. Dupuis, M.P. Houry, T. Margotin, J. Zerenyi, J. Dudzik, J. Machowski, L. Martin, J. M. Rodriguez, E. Urretavizcaya, “Analysis and Damping of Inter-Area Oscillations in the UCTE/CENTREL Power system", in CIGRE session 2000, 2000, paper 38-113.

[17] H. W. Weber, F. Prillwitz, M. Hladky, H.-P. Asal, "Reality Oriented Simulation Models of Power Plants for Restoration Studies" Pergamon Control Engineering Practice, 9, pp. 805-811, Dec. 2000.

[18] W. D. Stevenson, Elements of Power System Analysis, New York: McGrawHill, 1986.

[19] Turkey. Energy Market Regulatory Authority of Turkey. Elektrik Piyasası Şebeke Yönetmeliği, Ankara: Energy Market Regulatory Authority of Turkey, 2003. Available: http://www.epdk.org.tr/mevzuat/yonetmelik/elektrik/sebeke/sebeke.pdf. [Accessed May 31, 2010].

[20] IEEE Tutorial Course, "Power System Stabilization via Excitation Control," in IEEE Power Engineering Society General Meeting, 2007.

[21] O. Y1lmaz, "Participation of Combined Cycle Power Plants to Power System Frequency Control: Modeling and Application”, Ms Thesis, Middle East Technical University, Apr. 2006.

[22] O. Yılmaz, M. E. Cebeci, C. Gençoğlu, D. Gezer, "EÜAŞ Sır Hydroelectric Power Plant: Study Report on Field Tests for Evaluating Primary Frequency Control Performance," TUBITAK Space Technologies Research Institute Power Systems Group, Ankara, Turkey, Aug. 2009.

[23] O. Yılmaz, M. E. Cebeci, C. Gençoğlu, D. Gezer, "EÜAŞ Berke Hydroelectric Power Plant: Study Report on Field Tests for Evaluating Primary Frequency Control 
Performance," TUBITAK Space Technologies Research Institute Power Systems Group, Ankara, Turkey, Aug. 2009.

[24] O. Yılmaz, M. E. Cebeci, C. Gençoğlu, “Altınkaya Hydroelectric Power Plant: Field Test Study Report," TUBITAK Space Technologies Research Institute Power Systems Group, Ankara, Turkey, Oct. 2009.

[25] O. Yılmaz, M. E. Cebeci, C. Gençoğlu, "Hasan Uğurlu Hydroelectric Power Plant: Field Test Study Report,” TUBITAK Space Technologies Research Institute Power Systems Group, Ankara, Turkey, Oct. 2009.

[26] O. Yılmaz, M. E. Cebeci, C. Gençoğlu, “Atatürk Hidroelektrik Santrali Hız Regülatörü Ayarları ve Dikkate Alınması Gereken Hususlar,” TUBITAK Space Technologies Research Institute Power Systems Group, Ankara, Turkey, Nov. 2009.

[27] O. Yılmaz, M. E. Cebeci, C. Gençoğlu, "Karakaya Hidroelektrik Santrali Hız Regülatörü Ayarları ve Dikkate Alınması Gereken Hususlar," TUBITAK Space Technologies Research Institute Power Systems Group, Ankara, Turkey, Nov. 2009.

[28] O. Yılmaz, M. E. Cebeci, M. Altın, C. Gençoğlu, "Birecik Hydroelectric Power Plant: Field Test Study Report,” TUBITAK Space Technologies Research Institute Power Systems Group, Ankara, Turkey, June. 2009.

[29] O. B. Tor, O. Yılmaz, "Oymapınar Hydroelectric Power Plant: Tests on Unit 2 Governor and PSS," TUBITAK Space Technologies Research Institute Power Systems Group, Ankara, Turkey, Sep. 2008.

[30] Wikipedia, Water Turbine, 2010. [Online]. Available: http://en.wikipedia.org/wiki/Water_turbine. [Accessed: May 31, 2010].

[31] IEEE Recommended Practice for Excitation System Models for Power System Stability Studies, IEEE Std. 421.5, 2005. 
[32] O. B. Tor, U. Karaagac and E. Benlier, "Step Response Tests of a Unit at Atatürk Hydro Power Plant and Investigation of the Simple Representation of Unit Control System," IEEE Power Engineering Society, 36th North American Power Symposium, University of Idaho, Moscow, USA, August 9-10, 2004, MD-000336.

[33] "Preventive Measures for Effective Damping of Inter Area Oscillations after the Synchronous Interconnection of the Turkish Power System with the UCTE Power System”, UCTE, May 2009.

[34] Mathworks Company, http://www.mathwork.com, [Accessed May 31, 2010].

[35] Siemens Company, http://www.pti-us.com, [Accessed May 31, 2010].

[36] DIgSILENT Company, http://www.digsilent.de/, [Accessed May 31, 2010].

[37] Hangzhou Dalu Electric Equipment Company, http://en.lulutong.cc/prodetails.asp?tid=15\&id=8, [Accessed May 31, 2010]. 